\title{
The developments of multi-level computational methodologies for discrete element modelling of granular materials
}

\author{
Zhao, Tingting
}

How to cite:

Zhao, Tingting (2019) The developments of multi-level computational methodologies for discrete element modelling of granular materials. Doctoral thesis, Swansea University.

http://cronfa.swan.ac.uk/Record/cronfa52441

Use policy:

This item is brought to you by Swansea University. Any person downloading material is agreeing to abide by the terms of the repository licence: copies of full text items may be used or reproduced in any format or medium, without prior permission for personal research or study, educational or non-commercial purposes only. The copyright for any work remains with the original author unless otherwise specified. The full-text must not be sold in any format or medium without the formal permission of the copyright holder. Permission for multiple reproductions should be obtained from the original author.

Authors are personally responsible for adhering to copyright and publisher restrictions when uploading content to the repository.

Please link to the metadata record in the Swansea University repository, Cronfa (link given in the citation reference above.)

http://www.swansea.ac.uk/library/researchsupport/ris-support/ 


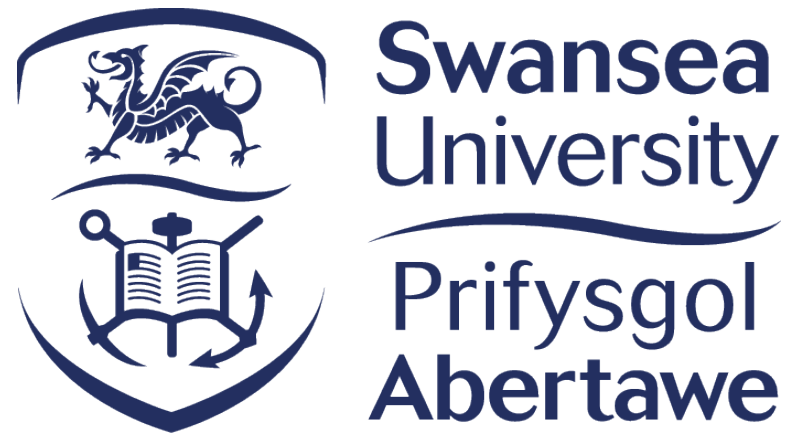

\section{The Developments of Multi-level Computational Methodologies for Discrete Element Modelling of Granular Materials}

Tingting Zhao 


\begin{abstract}
Granular materials are prevalent in this world while their non-trivial behaviour, which may resemble solid, liquid and/or gas under different circumstances, is still poorly understood. The challenging mechanics and dynamics of granular materials combined with their ubiquity have made this topic especially interesting to study. The discrete element method (DEM) is a reliable and effective numerical technique to model many scientific and engineering problems involving granular materials but it is still not a fully mature method. Considering the unique properties of granular materials and the inadequate features of the DEM, this thesis improves the current DEM from three different aspects and scales.

On the micro scale at the particle level, a novel contact model is developed by introducing the statistical Greenwood Williamson (GW) model which can consider the stochastic surface roughness of particles. Two non-dimensional forms of the original formulations are derived which can reduce the computational costs significantly. A Newton-Raphson based numerical solution is proposed which can solve the inter-dependence problem involved. A theoretical inconsistency of the classic GW model is deduced which leads to the development of the extended elastic GW (E-GW) model. An empirical normal contact law is obtained by the curve-fitting method and can be incorporated into the DEM code to conduct the one and three dimension compression tests. An extended elastic-plastic GW (EP-GW) model is developed to allow the plastic deformation at the asperities. Furthermore, the tangential contact model and thermal conductivity model are proposed.

On the meso scale at the sample level, a new packing characterisation method is proposed based on the digitalised image matrix of a packing and the subsequent application of the principal component analysis (PCA) with which the configuration of the particle assemblies can be evaluated quantitatively. The procedures of the packing digitalisation and formation of packing image are established for both $2 \mathrm{D}$ and $3 \mathrm{D}$ cases. The obtained PCA results of the packing image matrix can be revealed by the proposed principal variance function (PVF) and dissimilarity coefficient (DC). The values of PVF and DC can indicate the magnitude of effects on a packing caused by the configuration randomness, the particle distribution, the packing density and the particle size distribution. The uniformity and isotropy of a packing can also be investigated by this PCA based approach.

On the macro scale at the level of real industrial applications, the existing coarse graining methods are carefully analysed by the exact scaling law and the effective thermal properties of particulate phase change materials are derived by the homogenisation method. An enthalpy based discrete thermal modelling framework for particulate systems with phase change materials is developed which can consider both the heat conduction process and the phase change transition. This proposed methodology is assessed by solving a particle version of the classic one-phase Stefan melting problem. Additional numerical simulations are also conducted to illustrate the effectiveness of this modelling framework.
\end{abstract}




\section{Declaration and Statements}

\section{DECLARATION}

This work has not previously been accepted in substance for any degree is not being concurrently submitted in candidature for any degree.

Signed

Date

\section{STATEMENT 1}

This thesis is the result of my own investigations, except where otherwise stated. Where correction services have been used, the extent and nature of the correction is clearly marked in a footnotes(s).

Other sources are acknowledged by footnotes giving explicit references. A bibliography is appended.

Signed

Date

\section{STATEMENT 2}

I hereby give consent for the thesis, if accepted, to be available for photocopying and for inter-library loan, and for the tile and summary to be made available to outside organisations.

Signed

Date 


\section{Publications}

\section{The following publications are based on the research presented in this thesis:}

T. Zhao and Y.T. Feng. An enthalpy based discrete thermal modelling framework for particulate systems with phase change materials. Powder Technology, 354:505-516, 2019.

T. Zhao and Y.T. Feng. Extended Greenwood-Williamson Models for Rough Spheres. ASME. Journal of Applied Mechanics, 85(10):101007-101007-9, 2018.

T. Zhao, Y.T. Feng and M. Wang. An extended Greenwood-Williamson model based normal interaction law for discrete element modelling of spherical particles with surface roughness. International Journal for Numerical Analytical Methods in Geomechanics, 42:1624-1642, 2018.

T. Zhao, Y.T. Feng, M. Wang and Y. Wang. The modified Greenwood-Williamson model based stochastic discrete element method for contact with surface roughness. Rock and Soil Mechanics, 39(9): 3440-3452, 2018 (In Chinese).

Y.T. Feng, T. Zhao, M. Wang and DRJ. Owen. Characterising particle packings by principal component analysis. Computer Methods in Applied Mechanics and Engineering, 340: 70-89, 2018.

Y.T. Feng, T. Zhao, J. Kato and W. Zhou. Towards stochastic discrete element modelling of spherical particles with surface roughness: A normal interaction law. Computer Methods in Applied Mechanics and Engineering, 315: 247-272, 2017.

Y.T. Feng, T. Zhao, J. Kato and W. Zhou. Stochastic discrete element modelling of rough particles - a random normal interaction law. Jisuan Lixue Xuebao/Chinese Journal of Computational Mechanics, 33(4): 629, 2016.

T. Zhao and Y.T. Feng. Characterising 3D spherical packings by principal component analysis. Engineering Computations. In press. 


\section{Contents}

$\begin{array}{ll}\text { Abstract } & \text { i }\end{array}$

Declaration and Statements $\quad$ ii

$\begin{array}{lll}\text { Publications } & \text { iii }\end{array}$

Contents $\quad$ iv

Acknowledgement $\quad x$

List of Tables $\quad$ xii

List of Figures $\quad$ xiv

I Background 1

1 Introduction 2

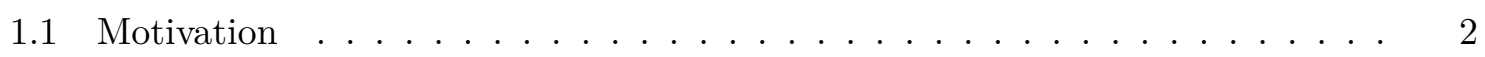

1.1.1 Granular material . . . . . . . . . . . . . . . . . 2

1.1.2 Discrete element $\operatorname{method} \ldots \ldots \ldots \ldots \ldots$

1.2 Scope of the current research $\ldots \ldots \ldots \ldots \ldots$

1.2.1 Surface roughness and contact model . . . . . . . . . . . . . . . 5

1.2.2 Packing features and characterisation methods . . . . . . . . . 6

1.2.3 Largescale problem and multiscale modelling . . . . . . . . . . 6

1.3 Layout of the thesis $\ldots \ldots \ldots \ldots \ldots \ldots \ldots$

2 Discrete element method $\quad 9$ 


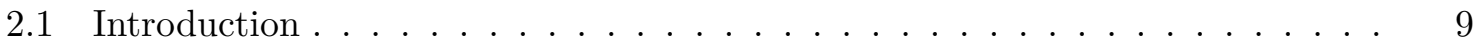

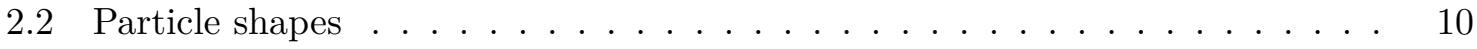

2.2 .1 Disk and sphere . . . . . . . . . . . . . . . . 11

$2.2 .2 \quad$ Ellipse and ellipsoid f . . . . . . . . . . . . . . . . . . . 12

2.2.3 Superquatratic and superquadric . . . . . . . . . . . . . . . . 12

2.2 .4 Polygonal and polyhedral . . . . . . . . . . . . . . . . . 13

2.2 .5 Agglomerate . . . . . . . . . . . . . . . . 13

2.3 Contact detection . . . . . . . . . . . . . . . . . . . . . 14

2.3.1 The global contact search . . . . . . . . . . . . . . . . . . 14

2.3 .2 Local resolution check . . . . . . . . . . . . . . . . . . 16

2.4 Contact models . . . . . . . . . . . . . . . . . . . . . . . . 16

2.4.1 Rheological model . . . . . . . . . . . . . . . . . . . . . 16

2.4.2 Normal contact models . . . . . . . . . . . . . . . . . . . 17

2.4 .3 Tangential contact models . . . . . . . . . . . . . . . . 18

2.4 .4 Rolling resistance models . . . . . . . . . . . . . . . . . . . . 19

2.4.5 Bond models . . . . . . . . . . . . . . . . . . . . . 21

2.5 Dynamic solutions . . . . . . . . . . . . . . . . . . 22

2.5 .1 Governing equations . . . . . . . . . . . . . . . 23

2.5 .2 Time integration . . . . . . . . . . . . . . . 23

$2.5 .3 \quad$ Accuracy and stability . . . . . . . . . . . . . . . . 24

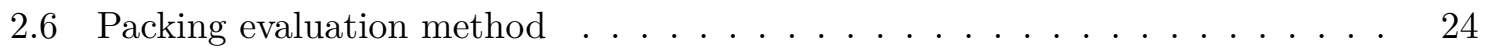

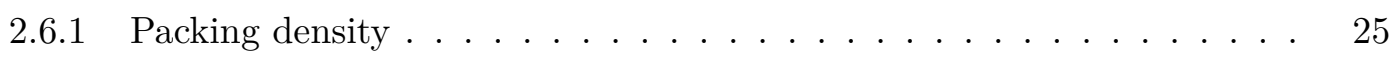

2.6 .2 Radial distribution function . . . . . . . . . . . . . 26

2.6 .3 Coordination number . . . . . . . . . . . . . 26

2.6 .4 Contact force distribution . . . . . . . . . . . . . 28

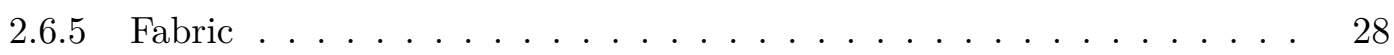

2.7 Multiscale modelling . . . . . . . . . . . . . . . . . . 30

2.7 .1 Coarse graining . . . . . . . . . . . . . . . . . . 31

2.7 .2 Combined FEM-DEM $\ldots \ldots \ldots \ldots \ldots$

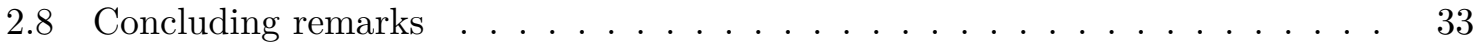


3 Surface roughness modelling - I. Classical GW model 35

3.1 Introduction . . . . . . . . . . . . . . . . . . . 35

3.2 The classic GW model . . . . . . . . . . . . . . . . . . . . . . . . . 36

3.2 .1 Characteristics of rough surfaces . . . . . . . . . . . 36

3.2 .2 Contact of two nominally flat rough surfaces . . . . . . . . . . . 38

3.2 .3 Contact of two rough spheres . . . . . . . . . . . . . . . 38

3.2.4 A simple extension to positive overlap and theoretical inconsistency . 40

3.3 Non-dimensional forms . . . . . . . . . . . . . . . . . . . . . . . 42

3.4 Numerical Solutions and Computational Issues . . . . . . . . . . . . . . . . . 44

3.4.1 Numerical solutions of the pressure and deformation distributions and the contact force . . . . . . . . . . . . . . . . . 45

3.4 .2 Computational issues . . . . . . . . . . . . . . . 46

3.5 Numerical Results and Validation . . . . . . . . . . . . . . . . 49

3.5.1 Convergence of the Newton-Raphson procedure . . . . . . . . . . . . . 49

3.5.2 Selections of numerical parameters . . . . . . . . . . . . . . . 49

3.5.3 Comparisons of pressure distributions and effective contact radii . . . 50

3.5.4 Effects of input parameters on pressure distributions . . . . . . . . . 52

3.6 Concluding remarks $\ldots \ldots \ldots \ldots \ldots \ldots \ldots$

4 Surface roughness modelling - II. Extended GW models $\quad 55$

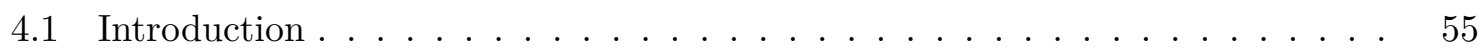

4.2 The extended elastic GW model . . . . . . . . . . . . . . . 55

4.2 .1 Model description . . . . . . . . . . . . . . . . . . 56

4.2.2 A normal interaction law based on the E-GW model . . . . . . . . . 59

4.2 .3 Numerical Illustrations of the E-GW model . . . . . . . . . . . . . . 64

4.3 The extended elastic-plastic GW model . . . . . . . . . . . . . . . . . . 73

4.3.1 Plastic contact model . . . . . . . . . . . . . . . . . 73

4.3 .2 Model description . . . . . . . . . . . . . . . . . . 75

4.3.3 Effects of plastic parameters . . . . . . . . . . . . . 77

4.4 The extended tangential GW model . . . . . . . . . . . . . . . . . . . 78 
4.4 .1 Tangential contact force . . . . . . . . . . . . . . 78

4.4 .2 Model description . . . . . . . . . . . . . . . . . . . 80

$4.4 .3 \quad$ Numerical results . . . . . . . . . . . . . . . . . . . . . . . 81

4.5 The extended thermal conductivity GW model . . . . . . . . . . . . . . . 83

4.5.1 Thermal resistance of rough surfaces . . . . . . . . . . . . . 83

4.5.2 Model description . . . . . . . . . . . . . . . . . . . . 87

$4.5 .3 \quad$ Numerical results . . . . . . . . . . . . . . . . . . . . . . . . 88

4.6 Concluding remarks $\ldots \ldots \ldots \ldots \ldots \ldots$

$\begin{array}{llr}\text { III } & \text { Packing characterisation methods } & 89\end{array}$

5 Characterising particle packings by PCA-2D $\quad 90$

5.1 Introduction . . . . . . . . . . . . . . . . . . . . . . 90

5.2 Principal Component Analysis _. . . . . . . . . . . . . . . . . . . 91

5.2.1 Packing digitalisation and formation of packing image . . . . . . . 92

5.2.2 Formulations and Numerical Procedures . . . . . . . . . . . . . . . . . 92

5.2 .3 Principal Variance Function . . . . . . . . . . . . . . . . . . . . . 95

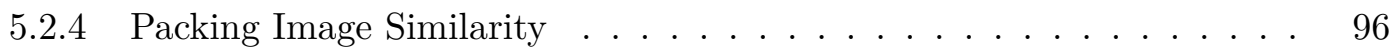

5.3 Principal Variances and Modes of Some Special Packings . . . . . . . . . . . 96

5.3.1 Permuted, Mirrored Packings . . . . . . . . . . . . . . . . 96

5.3.2 Repetitive, Periodic and Symmetric Packings . . . . . . . . . . . . . 97

5.3.3 Numerical Validation . . . . . . . . . . . . . . . . . . . . . . 98

5.4 Packing Characterisation using Principal Variances . . . . . . . . . . . . . . . 101

5.4.1 Numerical Examples: Two Sets of Random Packings . . . . . . . . . . 101

5.4.2 Dis/Similarity between Packing Samples and Groups . . . . . . . . . . 103

5.4 .3 Packing Uniformity and Isotropy . . . . . . . . . . . . . . . . 105

5.4 .4 Packing Density Effects _. . . . . . . . . . . . . . . 108

5.4.5 Principal Variances via Grid Resolutions . . . . . . . . . . . . . . . . 109

5.4.6 Principal Variances for Scaled Random Packings . . . . . . . . . . . . 110

5.5 Concluding remarks $\ldots \ldots \ldots \ldots \ldots \ldots \ldots$

6 Characterising particle packings by PCA-3D 113 
6.1 Introduction . . . . . . . . . . . . . . . . . . . . . 113

6.2 Principal component analysis . . . . . . . . . . . . . . . . . . . 113

6.2.1 Packing digitalisation and formation of packing image . . . . . . . 114

6.2.2 Formulations and numerical procedures . . . . . . . . . . . . 115

6.2.3 Different Schemes to compute the volume average of each cubic cell . 116

6.2 .4 Properties of repetitive packings $\ldots \ldots \ldots \ldots$

6.2.5 Analysis Region and Formation of Packing Matrix . . . . . . . . . . 118

6.2.6 Evaluation of main principal variances . . . . . . . . . . . . . . . 119

6.3 Packing Characterisation . . . . . . . . . . . . . . . . . 119

6.3 .1 Numerical samples . . . . . . . . . . . . . . . . . . . . . . . . . . 119

6.3.2 Different effects on packing samples . . . . . . . . . . . . . 120

6.3.3 Packing uniformity and isotropy . . . . . . . . . . . . . . . . . . . 124

6.4 Packing Evolution of a Particle System Subject to Tri-axial Loading . . . . . 127

6.5 Concluding remarks . . . . . . . . . . . . . . . . . . . . 129

$\begin{array}{ll}\text { IV Multiscale modelling } & 130\end{array}$

$\begin{array}{lll}7 & \text { Exact scaling laws and coarse graining methods } & 131\end{array}$

7.1 Introduction . . . . . . . . . . . . . . . . . . . . . . . . 131

7.2 Scaling conditions for exact scaled system . . . . . . . . . . . . 133

7.3 Coarse graining methods revisited f . . . . . . . . . . . . . . . 134

7.3.1 Representative model . . . . . . . . . . . . . . . . . 135

7.3 .2 Dimensionless analysis . . . . . . . . . . . . . . . . . 140

7.3.3 Modification of interaction laws . . . . . . . . . . . . . . . . 142

7.4 Concluding remarks $\ldots \ldots \ldots \ldots \ldots \ldots$

8 Effective thermal properties of particulate phase change materials $\quad 144$

8.1 Introduction . . . . . . . . . . . . . . . . . . . . . . 144

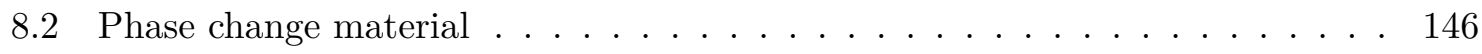

8.2.1 Encapsulation of PCM . . . . . . . . . . . . . . . 146

8.2 .2 Materials characterisation . . . . . . . . . . . . . . 147

8.2 .3 Mathematical models . . . . . . . . . . . . . . . . . 147 
8.3 The enthalpy based DTEM framework . . . . . . . . . . . . . . . . 148

8.3.1 Heat conduction modelling . . . . . . . . . . . . . . . . 148

8.3 .2 Phase change modelling . . . . . . . . . . . . . . . . . . . 151

8.4 Effective thermal properties . . . . . . . . . . . . . . . 152

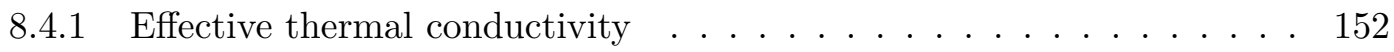

8.4 .2 Effective latent heat . . . . . . . . . . . . . . . . . . . 154

8.5 Validation . . . . . . . . . . . . . . . . . . 155

8.5.1 The Stefan problem _. . . . . . . . . . . . . . 155

8.5.2 Effective latent heat of a particle system based on DTEM . . . . . . . 157

8.6 Further Illustrations $\ldots \ldots \ldots \ldots$

8.6.1 Temperature evolution . . . . . . . . . . . . . . . . 159

8.6.2 Effect of capsule size and density . . . . . . . . . . . . . . . . 159

8.6.3 Determination of effective thermal conductivity . . . . . . . . . . . 161

8.6.4 Effects of thermal induced particle size and packing configuration changes162

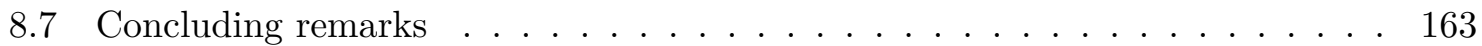

$\begin{array}{llr}\text { V Conclusion } & 164\end{array}$

9 Conclusion $\quad 165$

9.1 Major contributions . . . . . . . . . . . . . . . . 165

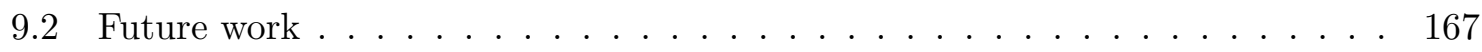

$\begin{array}{lr}\text { References } & 169\end{array}$ 


\section{Acknowledgement}

I wish to express my deepest gratitude and sincerest thanks to my supervisor, Professor Y.T. Feng, for his constant guidance, encouragement, patience and support throughout my PhD study. Prof. Feng is a genuine scholar with profound knowledge and broad mind. He is also the best supervisor who is always amiable and supportive. I learned a lot from every meeting and discussion with him during my PhD study which included discovering new research directions, solving baffling problems, understanding the basic theory deeply and even treating the technical details of programming. There are two sentences from Prof. Feng that impressed me most for the last four years. The first one is 'This is a good thing', which is suitable for all situations. It really made me happy when I obtained some desirable results; while I heard it more often when I got something mismatching the expectations because it meant we had a new problem to be solved. This first sentence gave me a lot of encouragement on my research and life. The second one is 'I had a new idea on my way cycling to the university' which is just like a magic sentence and give me endless surprise and confidence. The second sentence made me feel the happiness of finding and then solving scientific problems.

I also wish to express my greatest love to my dear parents. Their unreserved understanding, support and love give me the courage to continue all the time.

Thanks to the kindness of all the friends and strangers. 


\section{致谢}

博士阶段，最要感谢的人是我的导师冯云田教授，从他身上，我感受到了真正的学者风范。冯老 师学识渊博，思维开阔，平易近人，朴实无华。博士期间每次与冯老师的见面讨论，都让我受益 匪浅，或者找到了新的研究方向，或者解决了一直困惑的难题，或者对已有知识有了更加深刻的 认识，甚至是编程方面的细节问题，他也不厌其烦，耐心指导。四年中，冯老师有两句话说得最 多。第一句，“这是好事情！”。这句话适用于任何场合，当看到理想的结果时，听到这句话无 疑会让人感到心情舒畅；更多的时候，当结果与预期不符时，冯老师也常常会说这句话，因为我 们又有一个新的问题可以解决了。这种积极看待问题的方式，深刻影响了我自己对待科研甚至生 活的态度。第二句，“我在骑车来学校的路上又有了一个新想法！”。如果说第一句话还只是精 神上的鼓励，第二句话真的给了我太多的惊喜和信心，让我不断体会到发现问题解决问题的快乐。 有幸在冯老师指导下完成博士学业，实在是自己一生的财富。

其次，我更要感谢父母一直以来对我的养育和支持，他们对我毫无保留的爱与信任，是我前进的 最大动力。

最后，感谢一路走来所有熟人和陌生人的善意。 


\section{List of Tables}

3.1 The scaling factors in two non-dimensional forms . . . . . . . . . . . . . 43

3.2 The pressure errors in the Newton-Raphson iterations for different $\alpha(\mu=4) \quad 49$

3.3 The computed $P_{G}^{*}(\alpha, \mu)$ for different numbers of Gaussian points $m(\mu=4)$. $\quad 50$

4.1 Case I $(0 \leq \alpha \leq 1)$ : Coefficients of the cubic polynomial for different $\mu \ldots \ldots 1$

4.2 Case II $\left(0 \leq \alpha^{\prime} \leq 1\right)$ : Coefficients of the quadratic polynomial for different $\mu$. 63

4.3 Case III $\left(-3 \leq \alpha^{\prime} \leq 0\right)$ : Coefficients of the quartic polynomial for different $\mu \quad 64$

4.4 Percentage of the number of contacts number of initial packings (One-dimensional

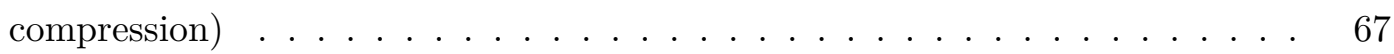

4.5 Percentage of the number of contacts of initial packings (Three-dimensional

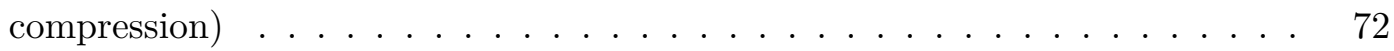

4.6 Parameters used in the contact models . . . . . . . . . . . . . . . . . 74

5.1 Total variances of three regular packings with different grid resolutions . . . . 98

5.2 The first principal variances of three regular packings with different grid resolutions 99

5.3 Some properties of two sets of random packings . . . . . . . . . . . . . 102

5.4 Average dissimilarity coefficient of each group in two sets of random packings 104

5.5 Dissimilarity coefficients between groups in two sets of random packings . . . 104

5.6 Dissimilarity coefficients of Submatrices of 4 packing images at N=1600 . . 106

5.7 Dissimilarity coefficients of two groups with two packing densities . . . . . . 109

6.1 The overall packing density of three regular packings computed by different computational schemes . . . . . . . . . . . . . . . . . . 117

6.2 The first principal variances of three regular packings computed by different computational schemes . . . . . . . . . . . . . . . . . 118

6.3 Properties of random packings: U-set and G-set . . . . . . . . . . . . . . . 120 
6.4 Packing Properties of the L-set . . . . . . . . . . . . . . 120

6.5 Average dissimilarity coefficients of each group in U-set and G-set . . . . . . . 121

6.6 Dissimilarity coefficients between different groups . . . . . . . . . . . . . 122

7.1 Scale factors for some physical quantities in the exact scaling system . . . . . 134

8.1 Effective latent heat of the particulate system . . . . . . . . . . . . . 159

8.2 Effective thermal conductivity of the particulate system $\ldots \ldots \ldots \ldots 1$ 


\section{List of Figures}

1.1 Examples of granular materials. . . . . . . . . . . . . . . . . 3

1.2 Layout of the thesis $\ldots \ldots \ldots \ldots \ldots \ldots \ldots$

2.1 Schematic diagram of DEM simulation . . . . . . . . . . . . . . 10

2.2 Superquadric geometries . . . . . . . . . . . . . . . . . . . . . 12

2.3 Bounding box representation $\ldots \ldots \ldots \ldots \ldots \ldots$

2.4 Graphical representation and load-deformation response of basic rheological models . . . . . . . . . . . . . . . . . . . 17

3.1 Topography of a rough surface $\ldots \ldots \ldots \ldots \ldots$. . . . . . . . . . 37

3.2 Profile heights and probability density of summits $\ldots \ldots \ldots \ldots$

3.3 Profile of the contact between a smooth sphere and a rough surface: $\delta \leq 0 \ldots 38$

3.4 Distribution of diagonal coefficients $\alpha_{i i} \ldots \ldots \ldots \ldots \ldots \ldots$

3.5 The ratio of the maximum contact pressure $p_{G}(0)$ to the maximum Hertzian pressure $p_{0}$ against $\alpha$ and $\mu$ : (a) comparison of the present work with Johnson's for $\alpha<1$ and two $\mu$; (b) the present work for wider ranges of $\alpha$ and $\mu \ldots$.

3.6 The ratio of the effective contact radius $a^{*}$ to the Hertzian contact radius $a_{0}$ against $\alpha$ and $\mu$ : (a) comparison of the present work with Johnson's for $\alpha<1$ and two $\mu$; (b) the present work for wider ranges of $\alpha$ and $\mu \ldots \ldots$.

3.7 Effective pressure distributions for different $\alpha$ and $\mu$ (the vertical line of each curve indicates the position of effective radius $\left.a^{*}\right) \ldots \ldots \ldots$

4.1 Profile of the contact between a smooth sphere and a rough surface: $\delta \geq 0 \ldots \quad 56$

4.2 Comparison of non-dimensional total contact forces between the GW and E-GW models for different degrees of roughness . . . . . . . . . . . . . . 57

4.3 Comparison of pressure distributions over the contact zone $\ldots \ldots \ldots$

4.4 Comparison of deformation distributions with over the contact zone . . . . 58 
4.5 The division of the $\delta-\sigma$ plane into three cases $\ldots \ldots \ldots \ldots$. . . . . . 60

4.6 Case I - $0 \leq \alpha \leq 1$ : (a) Computed $P^{*}$ and cubic polynomial fitted curves; (b) The coefficients of the cubic polynomial . . . . . . . . . . . . . 61

4.7 Case II - $0 \leq \alpha^{\prime} \leq 1$ : (a) Computed $P^{*}$ and quadratic polynomial fitted curves; (b) The coefficients of the quadratic polynomial . . . . . . . . . . . . . 62

4.8 Case III - $-3 \leq \alpha^{\prime} \leq 0$ : (a) Computed $P^{*}$ and quartic polynomial fitted curves; (b) The coefficients of the quartic polynomial . . . . . . . . . . . 63

4.9 Rough surfaces of particles with different roughness parameters . . . . . . 65

4.10 The normal force interaction laws for particles with different surface roughness

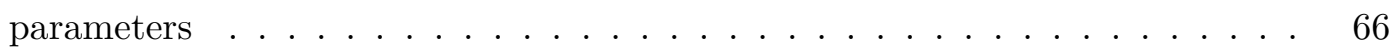

4.11 Numerical samples . . . . . . . . . . . . . . . . . . . . . 66

4.12 Porosities of the initial packings for samples with different roughness parameters 67

4.13 Normal contact links for initial packing samples with different roughness pa-

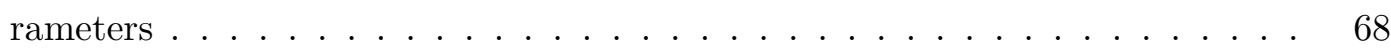

4.14 Normal contact distributions and contact laws for different samples . . . . . . 69

4.15 Results of seven different samples under one-dimensional compression . . . . 69

4.16 Stress-strain curves and corresponding normal contact density distribution: Axial strain $=0.1 \ldots \ldots \ldots \ldots$. . . . . . . . . . . . . . . . . . .

4.17 Stress-strain curves and corresponding normal contact density distribution:

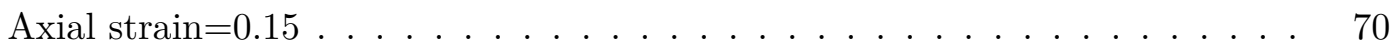

4.18 Stress-strain curves and corresponding normal contact density distribution:

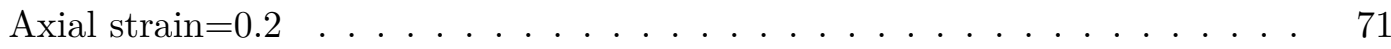

4.19 Relation between percentage of the number of contacts and axial strain for three cases . . . . . . . . . . . . . . . . . . . . 71

4.20 Results of seven different samples under three-dimensional compression $\ldots$. . 72

4.21 Relation between percentage of the number of contacts and axial strain for three cases . . . . . . . . . . . . . . . . . . . . . . 72

4.22 Force displacement relationships based on different contact models . . . . . . 74

4.23 Comparisons of the pressure distribution between different contact models $\quad$. $\quad 77$

4.24 Comparisons of the force-displacement relationship between different contact

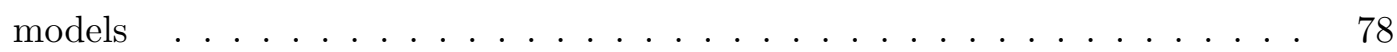

4.25 The schematic diagram of the Coulomb model . . . . . . . . . . . . . . 79

4.26 Contact between sphere and rough surface with normal and tangential displacement . . . . . . . . . . . . . . . . . . . . 81 


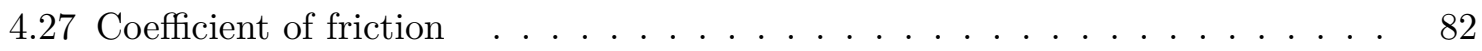

4.28 Pressure distribution . . . . . . . . . . . . . . . . . . . . . . 82

4.29 Coefficient of friction under different conditions . . . . . . . . . . . . 83

4.30 Variation of gradient of friction . . . . . . . . . . . . . 83

4.31 Variation of the coefficient of friction . . . . . . . . . . . . . . . 84

4.32 Conduction of nonconforming rough surfaces $\ldots \ldots \ldots$. . . . . . . 84

4.33 Thermal resistance network for nonconforming rough contacts in a vacuum . $\quad 85$

4.34 Microcontacts distribution in contact area and thermal resistance network for

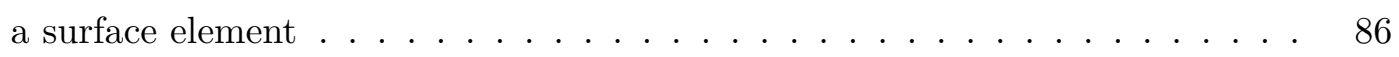

4.35 Thermal conductance based on different models . . . . . . . . . . . . . . 88

5.1 A random particle packing and a digital representation within the analysis window . . . . . . . . . . . . . . . . . . . 93

5.2 Three regular packings (a-c) and their digitalised images (d-f) $(\mathrm{N}=100) \ldots 9$

5.3 Principal variances of $\mathrm{R} 1, \mathrm{R} 4$ and $\mathrm{R} 16$ at $\mathrm{N}=100 \ldots \ldots$. . . . . . 100

5.4 Principal variances of $\mathrm{R} 1$ at $\mathrm{N}=25,50,100 \ldots \ldots \ldots \ldots$

5.5 Some leading principal modes of R1, R4 and R16 at N=100 . . . . . . . . 101

5.6 U-set: Four uniform packing groups in region $[-0.1,1.1] \times[-0.1,1.1](\mathrm{a}-\mathrm{d})$; and their digital images (with $\mathrm{N}=100)$ within region $[0,1] \times[0,1](\mathrm{e}-\mathrm{h}) \ldots \ldots 2$

5.7 G-set: Four Gaussian packing groups in region $[-0.1,1.1] \times[-0.1,1.1](\mathrm{a}-\mathrm{d})$; and their digital images (with $\mathrm{N}=100)$ within region $[0,1] \times[0,1](\mathrm{e}-\mathrm{h}) \ldots 103$

5.8 First three principal modes of $\mathrm{U} 1$ and G1 at $N=100 \ldots \ldots \ldots \ldots$

5.9 Average principal variance functions of groups U1 and G1 for three resolutions:

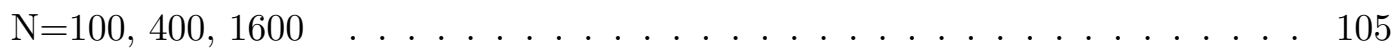

5.10 Dissimilarity coefficients of four packing groups U1, U4, G1 and G4 with four different image resolutions $N=100,200,400$, and $800 \ldots \ldots \ldots$

5.10 Dissimilarity coefficients of four packing groups U1, U4, G1 and G4 with four different image resolutions $N=100,200,400$, and 800-Continued $\ldots \ldots$. . 107

5.11 Principal coefficients of four images U1, U4, G1, G4 at N=1600 with three different sub-matrix blocks: $800 \times 800,400 \times 400$, and $200 \times 200 \ldots \ldots$

5.12 Dissimilarity coefficient distributions at randomly selected positions of groups U1, G1, U4 and G4 for 3 sizes of sub-matrices: N=800, 400, 200 . . . . . . 109

5.12 Dissimilarity coefficient distributions at randomly selected positions of groups U1, G1, U4 and G4 for 3 sizes of sub-matrices: N=800, 400, 200-Continued . 110 
5.13 Isotropic check for G1 using a rotating analysis window . . . . . . . . . 111

5.14 Packing anisotropic checking in two directions . . . . . . . . . . . . . . 111

5.15 Comparison of the principal variance functions and dissimilarity coefficients of scaled packing groups with different resolutions but with the ratio $r / h$ kept the same . . . . . . . . . . . . . . . . . . . 112

6.1 A random particle packing and the corresponding digital representation . . . 114

6.2 A cylindrical analysis region with a circular cross section image . . . . . . . . 119

6.3 Sample Packings of both U-set and G-set . . . . . . . . . . . . . . . . . 121

6.4 A sample packing of the L-set . . . . . . . . . . . . . . . . . . . . 122

6.5 Average principal variance functions of groups U1 and G1 for $\mathrm{N}=100,200 \ldots 123$

6.6 Dissimilarity coefficients of groups U1 and G1 for $\mathrm{N}=100,200 \ldots \ldots \ldots$

6.7 Average principal variance functions of different groups . . . . . . . . . . . . . 124

6.8 Density profiles of the U1 packing with different resolutions . . . . . . . . . 125

6.9 Dissimilarity coefficients of different samples and groups . . . . . . . . . 126

6.10 Uniformity and isotropy checks of a packing . . . . . . . . . . . . . . 126

6.11 Packing uniformity checkings for $\mathrm{U} 1$ and $\mathrm{L} \ldots \ldots \ldots \ldots$. . . . . . . 127

6.12 Principal variances for isotropic checking . . . . . . . . . . . . . . 127

6.13 Dissimilarity coefficient for isotropic checking . . . . . . . . . . . . . . 128

6.14 (a)Three dimensional compression (b)Deviatoric stress and Dissimilarity coefficient (DC) evolution . . . . . . . . . . . . . . . . . . . . 128

7.1 The original system, the exact scaling system and the coarse graining system 132

7.2 Diagram of the scaling law for the similar particle assembly (SPA) model . . 136

7.3 Coarse grain model . . . . . . . . . . . . . . . . . . . . . 138

8.1 Energy storage materials . . . . . . . . . . . . . . . . . . 146

8.2 Discrete thermal element method . . . . . . . . . . . . . . . . . . 151

8.3 Temperature distributions and time evolutions of the particulate system with PCM capsules . . . . . . . . . . . . . . . . . . . . . . . . . . . 158

8.4 Determination of the effective latent heat (Sample 1) . . . . . . . . 158

8.5 Temperature profiles and evolutions of the particulate system with two different materials at different time instances . . . . . . . . . . . . . . . 160 
8.6 Temperature evolutions of the particulate system with two different materials at different heights . . . . . . . . . . . . . . . . . . 160

8.7 Melt fraction evolutions of the particulate system with different particle sizes and packing densities . . . . . . . . . . . . . . . . . . . . . 161

8.8 Steady-state particle temperature distribution (a) and heat flux evolutions at the boundaries $($ b) for Sample $1 \ldots \ldots$. . . . . . . . . . . . . . 162

8.9 Final temperature distribution (a) and phase change time (b) for different packings with or without size change effect $\ldots \ldots \ldots 163$ 
Part I

Background 


\section{Chapter 1}

\section{Introduction}

\subsection{Motivation}

\subsubsection{Granular material}

This is a granular world, as it has always been. Granular materials are prevalent in the world from micro scale to macro scale, from daily life to industry. In the past millennia, we have used granular materials to measure time, to feed ourself and to build houses. While the non-trivial behavior of granular materials, which may resemble solid, liquid or gas under different circumstances, is still poorly understood. The challenging mechanics and dynamics of granular materials combined with their ubiquity have made this topic especially interesting to study. Granular materials have been extensively studied by both the scientific and engineering communities, such as applied mathematics, condensed matter physics, geomechanics, agriculture, chemical engineering and civil engineering [1].

For a long time, granular materials are studied on the macroscopic level by continuum solid mechanics. There are three independent assumptions in classical continuum mechanics, i.e. continuity, homogeneity and isotropy [2]. Granular materials consist of grains and surrounding voids thus their behaviour is inherently discontinuous and heterogeneous, and generally anisotropic. It is difficult to determine a constitutive model for granular materials based on continuum mechanical analyses. A constitutive model based on continuum approaches usually includes many material constants (or model parameters), which sometimes have no clear physical meaning [3]. The classical Mohr-Coulomb theory $[4,5]$ and continuous constitutive models of critical state $[6,7]$ or elastic-plasticity [8] are proposed to describe granular materials. These models are believed to describe stresses well in static granular materials but fail to predict their flow profiles. Furthermore, granular materials exhibit many interesting collective phenomena, such as pressure dip [9], density relaxation [10, 11], jamming [12], and force chains [13] which cannot be explained by the continuum theory. The shortcomings of the continuous approach may origin in the implicit expression of the geometry of a packed assembly of particles. Therefore, one can expect to analyse granular materials in a more realistic way by 


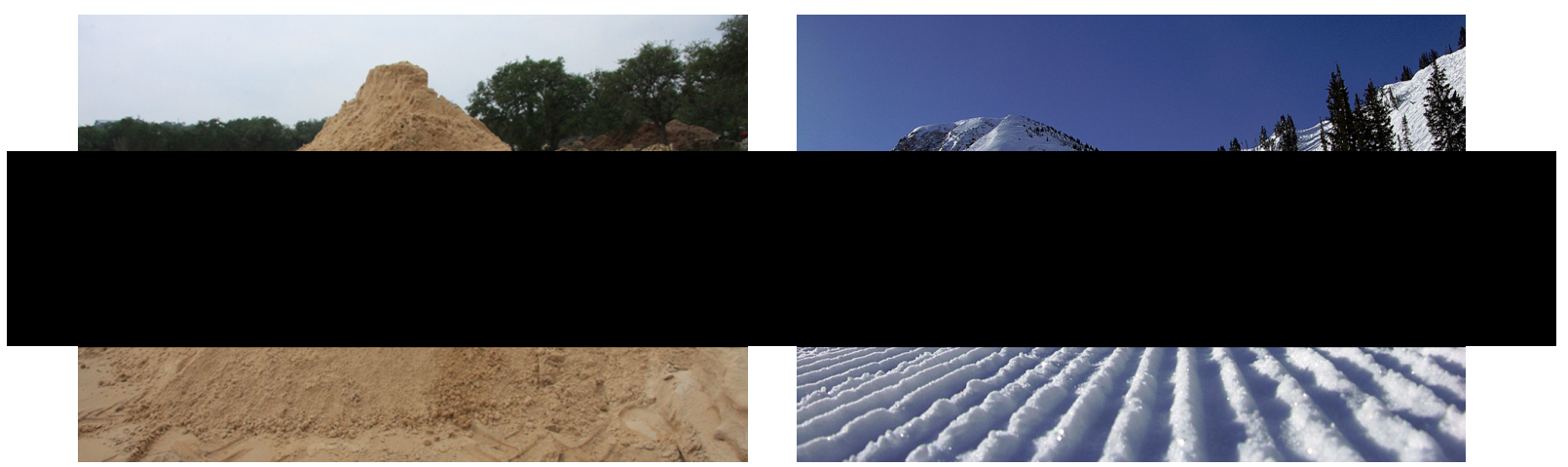

(a) Sand

(b) Snow

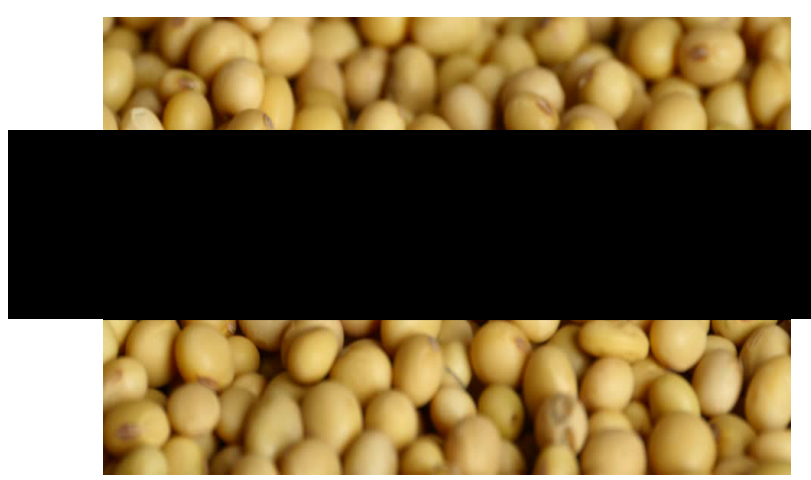

(c) Soybean

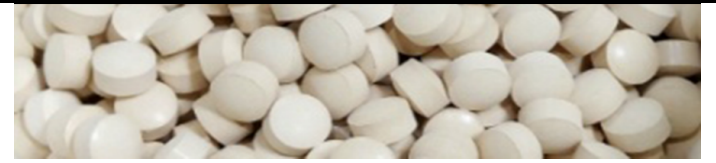

(d) Tablet

Figure 1.1: Examples of granular materials.

the discontinuous approach in which the particle arrangement is modelled explicitly. These two approaches are distinguished by Feda [14] as the phenomenological approach (structure-less continuum approach) and the structural (sometimes called micromechanical) approach. The structural approach can probe into the physical basis.

In discussing the analysis of granular materials at the micro scale, the classical $n$-body problem has attracted the attraction of physicists for hundreds of years which is similar to the interaction of a large system of particles [15]. The $n$-body problem is proposed to understand dynamics of the solar system which can be considered as the evolution of a system of $n$ bodies subject to Newtonian gravitational forces. There is no general closedform solution to this problem for systems with more than 3 bodies. In recent years, there has been a rapidly growing interest in studying the granular materials on the microscopic level mainly through the laboratory experiments and the numerical simulations. In terms of the laboratory experiments, measurement methods have been improved by developing novel equipments by which more internal structure informations of the granular materials can be observed comprehensively and exactly. It can provide detailed particle position and contact point maps. The most commonly used techniques include the photoelastic test [16-18], the scanning electron microscope [19], the X-ray computed microtomography and digital image correlation [20-23]. With the rapid development of the computer technology, it is possible to simulate granular materials at the discrete particle level. Several particle based 
discontinuous numerical methods are proposed which allow the inspection of every single body. Typical representatives of particle scale methods are SPH (Smooth Particle Hydrodynamics, for calculations of astrophysical phenomena or fluids [24, 25]), Monte Carlo method, LBM (Lattice Boltzmann Method, for calculations of fluids [26-29]), MD (Molecular Dynamics, for calculations of molecules and nanoparticles [30]) and DEM (Discrete Element Method).

In the Monte Carlo method [31], each particle is subject to a number of trial moves at each iteration. The change in energy generated by each of these moves is calculated and the movement leading to the lowest energy is selected for progressing to the next configuration. This approach is applicable only to the study of systems in static equilibrium, i.e. it cannot be applied to consider flow of granular materials. The molecular dynamics [32] simulates the interactions between individual molecules or atoms to relate the bulk properties of a material (liquid, solid or gas) to fundamental atomistic interactions. The point-like particles interact via pair or multi-particle interaction potentials. The time scale of interest in molecular dynamics are of the order of $1 \mu \mathrm{s}$, and the trajectory lengths are between 10 and 100 Angstroms [31]. Smooth particle hydrodynamics is another meshless method with the basic idea that the particles are used as interpolation points where the material displacement is tracked, and material is continuous between these points [33].

\subsubsection{Discrete element method}

\section{Development of DEM}

Since it was originated in the 1970s by the pioneer work of Cundall and Strack [34], the discrete element method (DEM) has emerged as a reliable and effective numerical technique to model many scientific and engineering problems involving granular materials. The development history of DEM can be found in the work of Jing and Stephansson [35]. Following the start of the original disk and sphere codes BALL and Trubal, several important improvements can be seen as the key milestones in the development of DEM including the hysteretic contact model [36], the non-spherical particles of ellipses and ellipsoids [37], the coupling of DEM with fluid [38], the development of bonded particulate modelling [39], and the implementation of DEM in a high-performance or parallel computing software environment [40-42].

The basic procedure of the DEM involves: (1) to represent particles as rigid geometric entities in various packing configurations; (2) to conduct contact detection to evaluate interaction forces between particles based on some appropriate physically based interaction laws; and (3) to assemble all the forces acting on each particle and to numerically solve the resulting dynamic equations of particles in the system to update their accelerations, velocities and positions at discrete time instants. This computational framework makes the DEM time consuming which limits the time and particle number of a simulation.

With advances in computing power and numerical algorithms for nearest neighbour sorting, it has become possible to numerically simulate millions of particles on a single processor. Today 
DEM has been widely accepted as an effective method of addressing engineering problems in granular and discontinuous materials. It has been applied in a wide range of industries, including agriculture and food handling [43], chemical industry [44], civil engineering [45-51], mining [52], pharmaceutical $[53,54]$ and so on. There are a series of software based on DEM which promotes its applications in different fields. The open-source and non-commercial software packages include YADE [55], LIGGGHTS [42] and EsysParticles [56]. Most popular commercial software are PFC [57], EDEM [58] and ELFEN [59].

\section{Challenges of DEM}

Despite the rapid developments and wide applications of DEM in recent years, it is still not a fully mature method which requires further analysis from different aspects. Simpson and Tatsuoka [60] and Yu [61] suggest that the future developments of DEM include the following:

1. Further parametric studies to consider the influence of the model parameters on the observed response.

2. More realistic contact models and particle morphologies.

3. An increase in the number of particles considered in simulations.

4. The development of more robust models and more efficient computer codes.

5. Improved micro-scale quantification of inter-particle forces and particle fluid interaction forces to inform future model development.

6. Improved theories to relate the macro- and micro-scales.

7. Further developments in relation to coupling particles and fluid, including simulation of multi-phase fluids.

\subsection{Scope of the current research}

Considering the unique properties of granular materials and some inadequate features of DEM, the current research improves the DEM from three different aspects and scales.

\subsubsection{Surface roughness and contact model}

Understanding the intrinsically random processes in nature is a fundamental question that has attracted the interest of multiple thinkers, ranging from philosophers and mathematicians to physicists or neuroscientists [62]. Uncertainties are encountered in engineering systems involving the aspects of the assessment of loading, material and geometric properties. Such as in most civil engineering applications, the intrinsic randomness of materials (soil, rock, concrete, ...) or loads (wind, earthquake, tide, ...) is a dominant factor to be considered. 
Thus data and models encountered in engineering problems should also be characterised by uncertainty. While the classical physics and mechanics are deterministic theories which implicitly assume that the results obtained from a deterministic analysis are representative of all possible scenarios of system. This is not true in most cases. Classical deterministic methods can provide only crude approximations for the response and evolution of the damage state of the system. In the last few decades, stochastic or probabilistic mechanics has developed fast which can account for randomness and spatial variability of the mechanical properties of materials [63].

Granular materials have been studied extensively because of their tremendous importance from different perspectives while very few attempts have been reported to investigate the stochastic properties of granular materials which may play a fundamental role on the performance of particulate systems.

Therefore, the first aim of this research is to investigate the influence of randomness of the particle surfaces. Surface roughness of particles has been considered by developing a novel contact model in DEM based on the classical Greenwood and Williamson (GW) model [64]. This part can be viewed as the improvement of DEM on the micro scale at the particle level.

\subsubsection{Packing features and characterisation methods}

The particle packing plays an important role in leading the physical behaviour of a particle system. Therefore the spatial-statistical analysis of the geometrical structure of the system is of great scientific and engineering interests. As the topology of the system is highly complex, it is difficult to observe the way particles packed around each other by experiments. Currently, the techniques applied to investigate the features of particle packings focus on the packing density, orientations of the particle contacts, and internal (topological) structures of packings. The conventional analyses have limits in some ways.

Considering the lack of more general, comprehensive and quantitative approaches which can reveal some fundamental features of packing, the second aim of this research is to develop a novel principal component analysis (PCA) based approach that can characterise particle packings by using their principal components or variances in both two dimension and three dimension.This part improves the understanding of results of DEM simulations on the meso scale at the sample level.

\subsubsection{Largescale problem and multiscale modelling}

Real industrial applications require billions of particles compared with the calculating capability of several million particles on a single personal computer. The simulation of a large number of particles requires unacceptable computational time that is the most critical problem existing in the industrial application of the DEM. Therefore, multiscale modelling techniques are required to tackle such large scale problems. 
The third aim of the current research is to treat issues related to the large scale problem from two perspectives of the coarse graining technique and the combined discontinuum and continuum method. Existing coarse graining techniques have been carefully analysed by the exact scaling law which can provide the theory basis for the upscaling method. Furthermore, by using the homogenisation method, the effective thermal properties of the phase change granular material can be derived by the DEM simulations. This part is devoted to solving the problems that occur in DEM simulations on the macro scale at the level of real applications.

\subsection{Layout of the thesis}

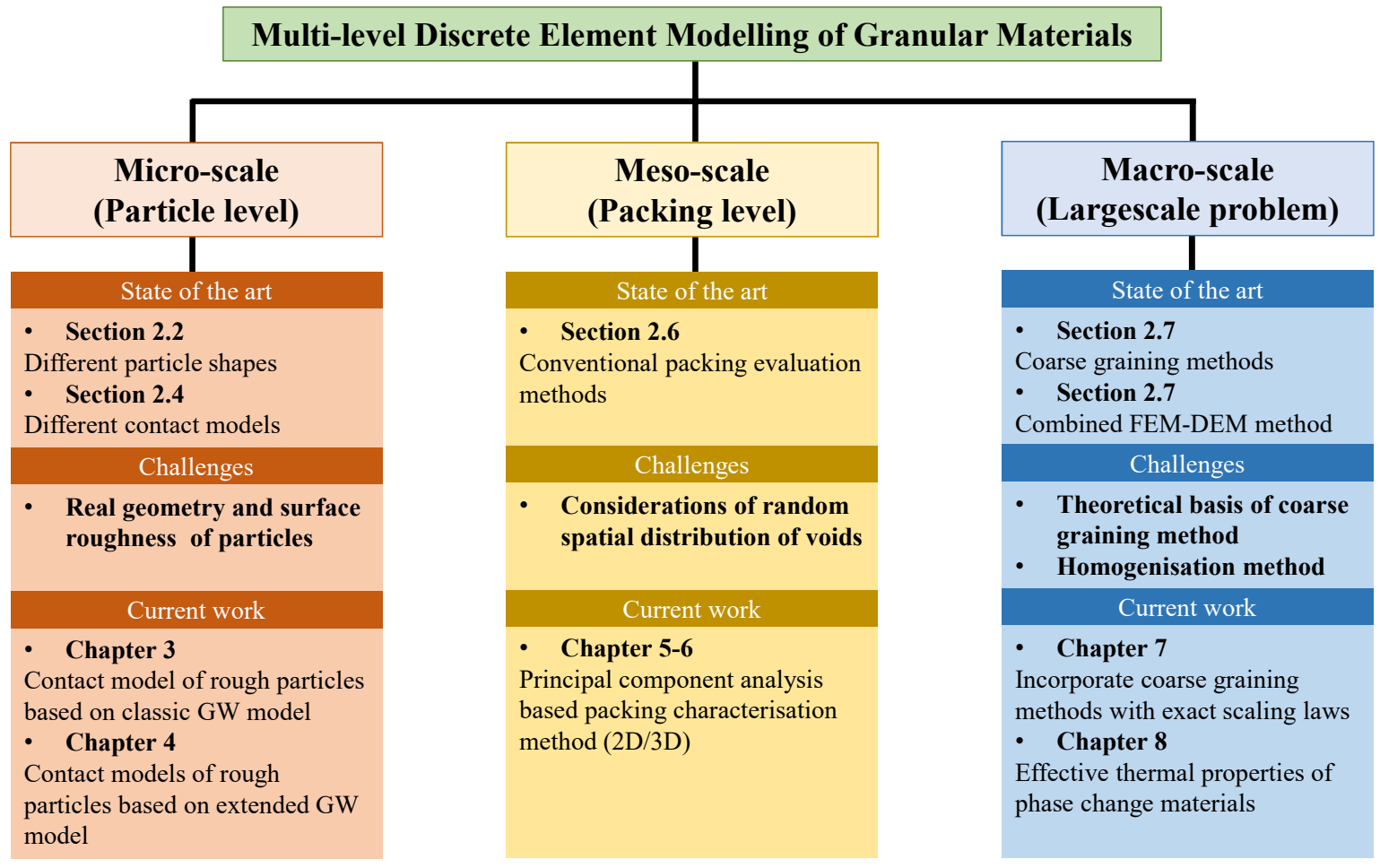

Figure 1.2: Layout of the thesis

This thesis contains five parts including nine chapters and is structured as follows. The main part of this thesis is shown in Figure 1.2.

Part I describes the background of the current research. Following the introduction in Chapter 1, Chapter 2 is devoted to providing the theoretical basis of the discrete element method and the literature review of the three problems involved in the current research.

Part II improves the DEM on the micro scale at the particle level by considering the surface roughness of particles. In Chapter 3, a novel normal contact model for rough particles is developed based on the classical Greenwood Williamson (GW) model. In Chapter 4, this developed normal contact model has been improved further based on the extended GW model. Simple extensions of this contact model to the tangential direction and thermal conduction have also been presented. 
Part III is devoted to making a better understanding of DEM simulation results on the meso scale at the packing level. A novel approach that can characterise particle packings by using their principal variances obtained from the principal component analysis is developed in Chapter 5 and its extension to three dimensional situation is presented in Chapter 6 .

Part IV attempts to solve the problems that occur in DEM simulations on the macro scale at the level of real applications. In Chapter 7, the exact scaling laws are applied to analyse the existing coarse graining methods. In Chapter 8 , an enthalpy based discrete thermal element method is developed based on which the equivalent thermal properties of bulk particle materials with phase change is derived.

Part V (Chapter 9) summarises the main work and innovations of this thesis and points out suggestions for the future work. 


\section{Chapter 2}

\section{Discrete element method}

\subsection{Introduction}

The discrete element method is a numerical approach suitable to simulate granular materials. The most unique feature of DEM is that individual particles and interactions can be considered explicitly which makes it possible to capture the mechanical response associated with granular materials. However, in the traditional continuum method, the granular material is assumed as a continuous material without considering the relative movements and rotations between particles. Then sophisticated constitutive models are needed to capture complexity of the material behaviour that arises owing to the particulate nature of the material.

Compared to the continuous method or the experiment method, two obvious advantages can be addressed in DEM in simulating the discontinuous material. Firstly, particle scale mechanisms that underlie the complex overall material response can be monitored and analysed. It is easy to measure the particle motion and contact evolution in DEM which are difficult to be accessed in the physical laboratory test. Secondly, DEM allows the analysis of the large-displacement problem which is very hard to be modelled using the continuum approach. Therefore, DEM simulations present us a valuable tool to understand the failure mechanisms and other problems involving the large displacement.

The numerical techniques involved in DEM can be divided into two categories $[65,66]$ : soft sphere model and hard sphere model. The major difference between the two models is whether penetration is allowed or not. The hard sphere model also called event driven (ED) model excludes the interpenetration during particles impact. The collision is assumed to be instantaneous. The momentum exchange is considered in the governing equation and the particle contact force is not considered explicitly. This hard sphere model is suitable to solve the problem involving rapid granular flow, e.g. avalanches, or rapid flow through conduits in manufacturing process. In the soft sphere model, overlap is allowed between contact particles. The governing equations are the linear and angular dynamic equilibrium of the contacting particles. It is worth noting that the DEM in the current work falls within the soft sphere category. 


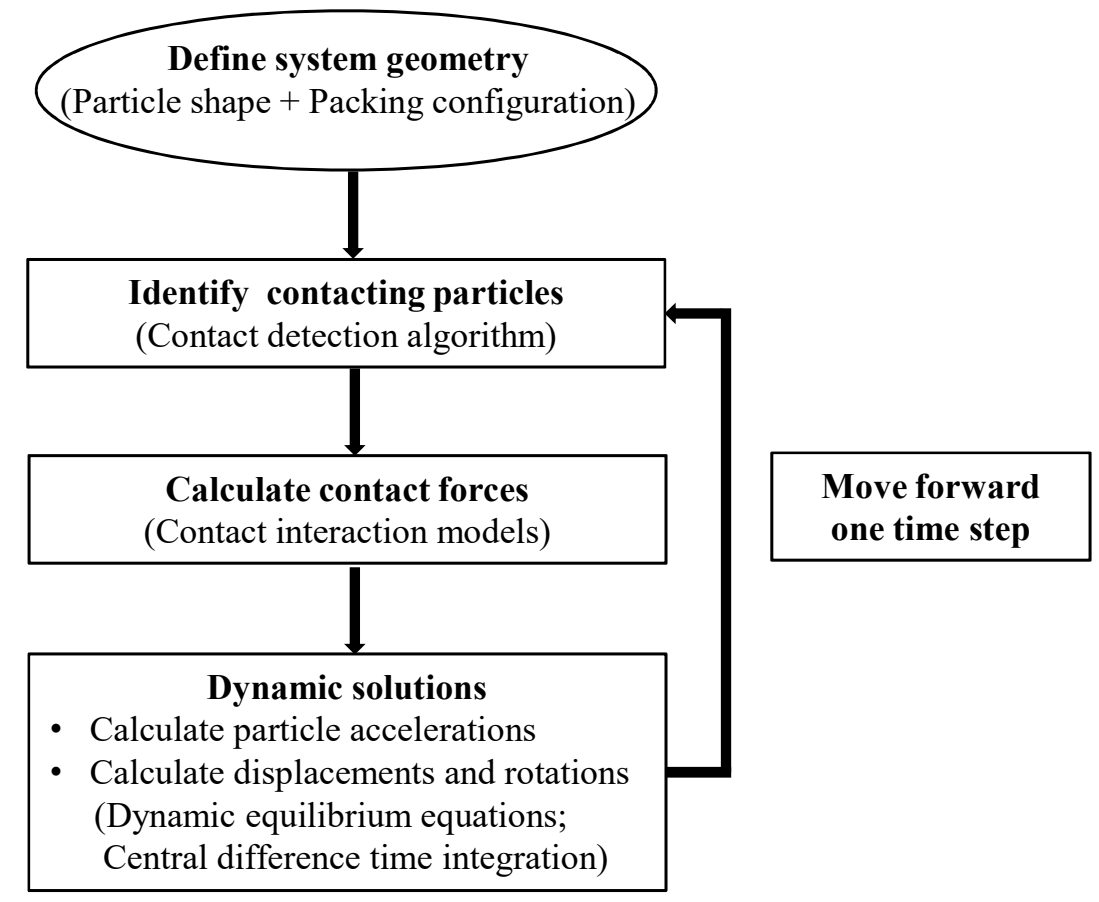

Figure 2.1: Schematic diagram of DEM simulation

The calculations sequence and corresponding theories involved in DEM simulation are shown in Figure 2.1. The first step is to define the system geometry, boundary condition, material property. Then with setting the loading schedule, the simulation progresses as a transient or dynamic analysis for a specified number of time increments. At each time step, the contacting particles are identified. Then the contact forces at the contact points are calculated based on the contact models which are used to determine the resultant force and moment acting on each particle. Knowing the particle mass and inertia, the translational and rotational accelerations of the particle can be calculated by solving the dynamic equilibrium equations. The displacement and rotation of the particle over the current time step is then found through the central difference type integration through time. Then the particle position and orientation will be updated according to these incremental displacement and rotation. This series of calculation will repeat at the next time step.

In this chapter, the basic knowledge of DEM and the theories related to the current work are introduced.

\section{$2.2 \quad$ Particle shapes}

The particle shape in DEM simulation is analytically defined which simplify the physical reality which is also the requirement to make the calculation involving large amount of particles efficiently. When using DEM, it is worth being aware of the extent of the simplifications and the associated implications. Furthermore, the particles are assumed as rigid bodies so only the translation of the particle centroids and the rigid body rotation need to be considered 
in the governing equilibrium equations. Therefore, for each particle, there are three degrees of freedom in 2D and six degrees of freedom in 3D excluding the consideration of particle deformations. Instead of considering the particle deformation directly, overlap is allowed when two particles contact. The contact forces are sensitive to the calculated overlap, the simple particle shapes facilitate the accurate determination of the contact geometry. Hogue [67] and Houlsby [68] discuss the issues related to the choice of particle geometry in DEM. It is better to achieve a balance between the benefits of improvement against the geometrical and numerical challenges associated with adding complexity and computational cost when choosing the particle shape.

\subsubsection{Disk and sphere}

Disks and spheres are the simplest and most common type of particles used in DEM. They are popular because it is easy to identify whether they are contacting and to determine the overlap or separation accurately. Thus the contact detection and resolution which is the most computationally expensive part of the DEM will become efficient to achieve a realistic number of particles in the simulations.

The contact overlap between two particles $a$ and $b$ is given by

$$
\begin{aligned}
& \delta_{n}=R_{a}+R_{b}-\sqrt{\left(x_{a}-x_{b}\right)^{2}+\left(y_{a}-y_{b}\right)^{2}} \\
& \delta_{n}=R_{a}+R_{b}-\sqrt{\left(x_{a}-x_{b}\right)^{2}+\left(y_{a}-y_{b}\right)^{2}+\left(z_{a}-z_{b}\right)^{2}}
\end{aligned}
$$

where $R_{a}$ and $R_{b}$ are the particle radii, and the centroid coordinates are given by $\left(x_{a}, y_{a}, z_{a}\right)$ and $\left(x_{b}, y_{b}, z_{b}\right)$. The contact overlap is taken to be positive in compression, otherwise the contact is considered inactive (unless it can transmit tension). The contact location $x_{i}^{c}$ is assumed to be at the midpoint of the contact overlap

$$
x_{i}^{c}=x_{i}^{a}+\left(R_{a}-\frac{\delta_{n}}{2}\right) n_{i}
$$

where $\mathbf{x}^{c}$ is the contact coordinates, $\mathbf{x}^{a}$ is the particle coordinates, and $\mathbf{n}$ is the contact normal which is defined by considering the position of $b$ relative to $a$

$$
n_{i}^{c}=\frac{x_{i}^{b}-x_{i}^{a}}{\left|x_{i}^{b}-x_{i}^{a}\right|}
$$

The calculations of masses and moments of inertia are also simple and straightforward. However, the ideal symmetrical and convex nature of disks or spheres leads to limitations in modelling the real material which includes the differences in shear strength, dilative response during shear and distributions of void space [69]. 


\subsubsection{Ellipse and ellipsoid}

One obvious drawback associated with using disk or sphere particles is the excessive rotations caused by the disappearance of moments due to the normal component of the contact force. When two disks contact, the branch vector (the vector connecting their centroids) and the contact normal are collinear so the normal contact force passes through the centroid of the disk without imparting any moment to the disk. This shortcoming can be overcame by using ellipse or ellipsoid particles of which the branch vector and the contact normal are no longer collinear. Elliptical particles for two-dimensional analyses were firstly proposed by Rothenburg and Bathurst [70] and Ting [71](1993) and then promoted by Dobry and Ng [72, 73].

\subsubsection{Superquatratic and superquadric}

Disk, sphere, ellipse and ellipsoid are all belong to a general type of functions called superquatratics $(2 \mathrm{D})$ or superquadrics (3D). Their general forms are defined as

$$
\begin{aligned}
& \left(\frac{x}{r_{a}}\right)^{n}+\left(\frac{y}{r_{b}}\right)^{n}=1 \\
& {\left[\left(\frac{x}{r_{a}}\right)^{\frac{2}{n}}+\left(\frac{y}{r_{b}}\right)^{\frac{2}{n}}\right]^{\frac{n}{m}}+\left(\frac{z}{r_{c}}\right)^{\frac{2}{m}}=1}
\end{aligned}
$$

where the principal axis lengths are given by $2 r_{a}, 2 r_{a}$, and $2 r_{c}$. The particle squareness is controlled by the exponents $m$ and $n$ as shown in Figure 2.2.

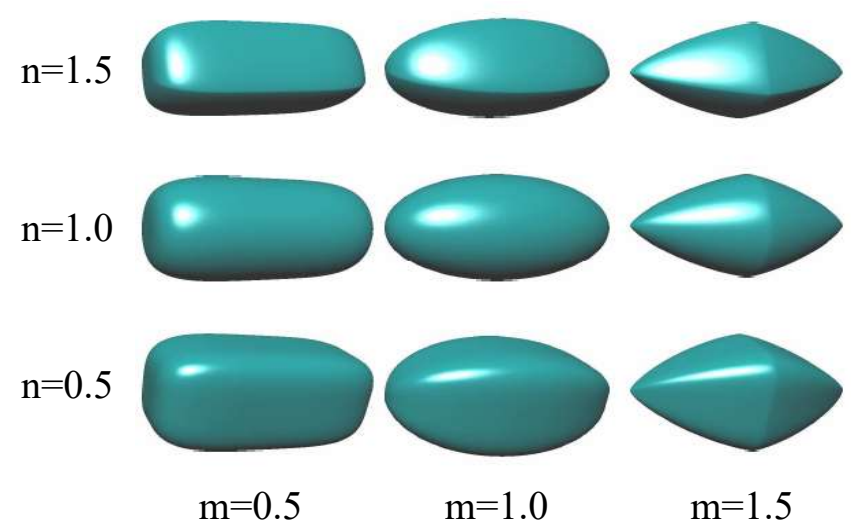

Figure 2.2: Superquadric geometries

If the surfaces of two particles $P_{1}$ and $P_{2}$ are defined by functions $f_{P_{1}}$ and $f_{P_{2}}$, the contact between them can be calculated using Lagrange multipliers. The point on $P_{1}$ that is closet to $P_{2},\left(x_{P_{1,2}}, y_{P_{1,2}}, z_{P_{1,2}}\right)$ is determined by minimising the sum $f_{P_{2}}+\Lambda f_{P_{1}}$ which can be achieved by differential calculus with an iterative Newton-Raphson method [68]. If $f_{P_{2}}\left(x_{P_{1,2}}, y_{P_{1,2}}, z_{P_{1,2}}\right)<$ 0 then the two particles contact with a positive overlap to calculate the contact force. In Hogue's work [67], another approach is adopted in which the surface of one particle is discretised and then each of these points is tested against the surface function of the adjacent particle. As the geometrical non-linearity increases, the computational cost grows significantly. 


\subsubsection{Polygonal and polyhedral}

The geometry of polygonal is defined by the vertex coordinates, the edges and the particle orientation. The volume of data required to describe a particle is approximately proportional to the number of vertices [67]. The coordinates of the particle centroid and orientation will be updated by the dynamic equilibrium equation during the calculation process.

Different contact types can be detected when using polygonal or polyhedral particles in DEM. Six potential types of contact for three dimensional polyhedral are identified by Cundall [74] including: corner-corner, corner-edge, corner-face, edge-edge, edge-face and face-face. A common plane approach is proposed for contact identification.

\subsubsection{Agglomerate}

The calculations of contact detection and resolution will be complex when using the shapes mentioned above other than disk or sphere. If disks or spheres are bonded together to simulate realistic geometries, the solution of a non-linear equation involved in the contact resolution will be avoided. Two types of agglomerate have been developed in DEM which are the rigid agglomerate and the crushable agglomerate.

Rigid agglomerate Disk or spherical particles can be glued together to create rigid clusters with non-smooth, non-convex and non-spherical geometries. The particles may either touch or overlap while the overlapping particles can model geometries with more complicated shape $[75,76]$. With the advances in optical microscopy and micro-computed tomography, the 3D morphological characterisation of particle geometry is accessible. Some algorithms have been developed to create agglomerate particles directly from the digital image of real particles which increases the level of sophistication of the particles shape significantly [77, 78].

As the agglomerate is regarded as a rigid body, no contact force is calculated between the particles making up this cluster even overlaps exist. The resultant force on the agglomerate is the sum of the contact forces on its constituent disks or spheres. The resultant moment is calculated by considering the cross product of each contact force and the vector directed from the contact point to the cluster centroid.

Crushable agglomerate If tensile or cohesive bonds are introduced between the particles in the cluster, then the agglomerate will become breakable which can model the phenomena of particle damage and crushing. When the forces between the base particles exceed the bond strength, the agglomerate will disintegrate into two or more smaller agglomerates. The contact forces acting on each of these base particles include contribution from contacts with spheres in adjacent agglomerates as well as contacts between the other particles in its own parent agglomerate. 


\subsection{Contact detection}

Referring to the computational procedure shown in Figure 2.1, the first step in calculating the contact forces is to identify the particles that are contacting in the current time step and develop the list of contacts in the system. The contact detection method is to determine the neighbours of each particle which are contacting or likely to contact with this target in a given time increment. Then each of these pairs of neighbouring particles will be considered in turn in the contact force calculation loop. Several notable features can be seen in the contact detection including [79]: a large number of discrete objects can be involved (e.g. $\sim 10^{6}$ or more); a wide variety of object shapes are presented; a large number of time instants are performed which make the contact detection computationally expensive and will take up to $80 \sim 90 \%$ of the total simulation time.

The most intuitive and naive approach to perform the contact detection process is to check each particle against all other particles in the system at each time increment based on their exact geometries. This method is prohibitively computationally expensive as the cost of the contact detection is proportional to $N_{p}^{2}$, where $N_{p}$ is the number of particles in the system, and thus the simulation time will increase significantly as the number of particles increases. What is more, for the particles of irregular shapes, it is also time consuming to determine the contacting neighbouring particles. Munjiza [80] stated that it is important to develop a contact detection algorithm with minimal CPU and memory requirements. A variety of researchers have devoted to the implementation of contact detection algorithms refer to Feng and Owen [81], Han et al. [82], Pöschel and Schwager [83] and Bobet et al. [84].

The contact detection is performed by two main steps as the global contact search and the local resolution check.

\subsubsection{The global contact search}

In this step, all the possible contacts in the whole domain are located by assigning a bounding box to each particle according to its specific shape. The global contact search is to determine the bounding boxes in contact with the selected target bounding box. It is more efficient to assess whether either two bounding boxes (shown in Figure 2.3) intersect at the contact detection stage, rather than resolving the contact geometry in detail.

Many algorithms are available to obtain the possible contacts [79]:

\section{Brutal search}

Check each object with every other object for contact. The memory requirement is $O\left(N_{p}\right)$ and the operations is $O\left(N_{p}^{2}\right)$.

\section{Tree based search}

There are also several tree based algorithms such as the point representation schemes, the tree data structures and the augmented spatial digital tree of which the details can be referred to Feng and Owen [81], Han et al. [82]. The memory requirement is $O\left(N_{p}\right)$ and the average 


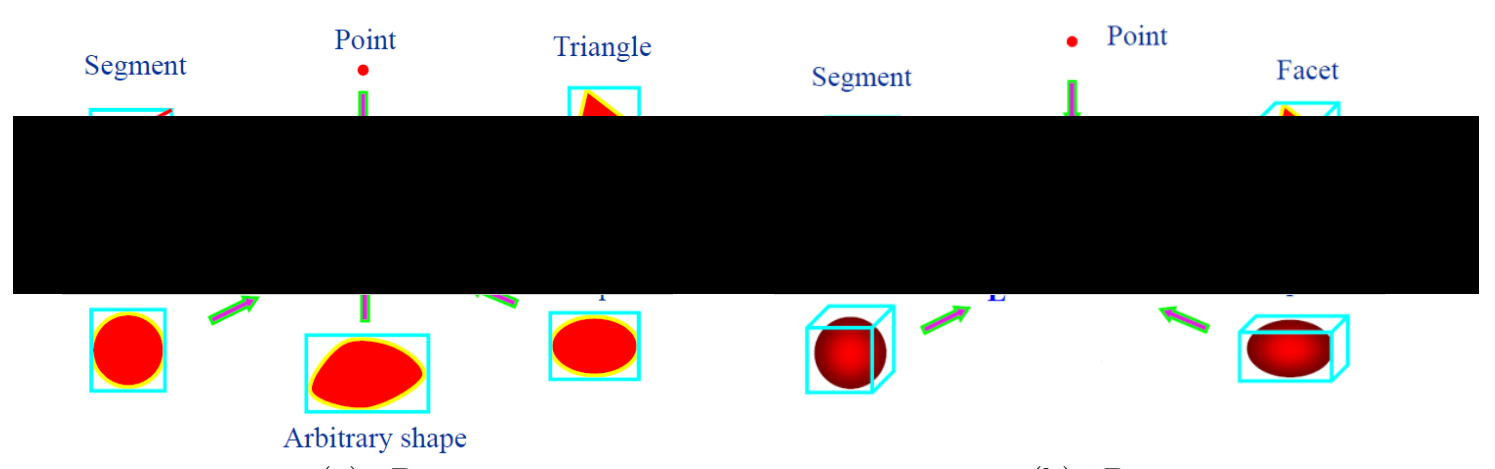
(a) $2 \mathrm{D}$
(b) $3 \mathrm{D}$

Figure 2.3: Bounding box representation enlarged with a buffer zone [79]

performance is $O\left(N_{p} \log \left(N_{p}\right)\right)$.

\section{Cell/Grid based search}

The most efficient algorithms are the grid based approaches which are relatively easy to implement and are commonly used which have the linear complexity with $O\left(N_{p}\right)$ of CPU and memory requirement.

Static: The domain is going to be divided into cells and each object is mapped to a cell based on the position of its corner or central point. The cell size must be no smaller than the maximum size of objects. Then loop over all cells in the search stage by checking the overlap between the objects within each cell and with those objects in the neighbouring cells. This method is simple and effective for small compact problems. The computer costs are proportional to the number of cells. While it is very computationally expensive for large simulations where the spatial distribution of objects is sparse and irregular.

No binary search $(N B S)$ : The mapping stage is the same to the previous static method. The following step is to create linked lists of particles that are in the same row of cells. For every linked list, check overlapping in its row and also the adjacent row. It avoids to loop over all cells in this approach and it dynamic processes two rows of cells at a time. It is very effective for large simulations and not sensitive to the spatial distribution of objects. The performance degrades, however, for objects with a wide range of size distribution.

Dynamic cell (D-cell): The domain is divided into rows. Each particle is assigned to a cell from the current row and they start to be processed from the left. An object is migrated to the following row if the upper $y$ coordinate is greater than its lower $y$ coordinate of the next row. These elements can also be migrated to the following cell if their upper $x$ coordinates are larger than their lower $x$ coordinate. This approach has a variety of features including no loop over cells, dynamic processing of cells with one row at a time, very effective for large simulations and not sensitive to the spatial distribution of objects and no performance degradation for objects with a wide range of size distribution. 


\subsubsection{Local resolution check}

It needs to be determined whether two particles are in contact according to the real shape when their bounding boxes overlap. Local contact resolution establishes if two discrete objects are in contact based on their actual geometric shapes. If the pair is in contact, the normal and tangential contact directions, the contact point and the characteristics of the overlap, such as the penetration, contact width and contact area may also need to be determined.

\subsection{Contact models}

To determine the contact force between contacting particles in DEM, two steps need to be done in series including contact detection and contact resolution [67]. For contact detection, efficient algorithms need to be developed to form the neighbour-list consisted of all the particles in contact. Contact resolution involves the accurate calculation of the contact geometry and kinematics. The overlap and tangential motion between particles are calculated first and then a contact constitutive model is applied to obtain the contact forces. The calculations involved in determining the contact forces is the most time consuming aspect in DEM as stated by Sutmann [31] that these calculations typically account for $70 \sim 80 \%$ of the total DEM simulation time. To make it possible to simulate the granular system with a large number of particles, a simple and straightforward expression to calculate the contact force is needed in DEM.

To facilitate the analytical description of the geometry, particles in DEM simulations are assumed to have smooth surfaces and the contacts occur at a single point with a specific overlap without considering the stress distribution and deformation at the contact area explicitly. As a result, the contact force is the equivalent substitution of the integral of the real stresses or tractions acting on a physical contact. Then, the stress-deformation response at the contact is represented using two orthogonal rheological models in the normal and tangential directions respectively which comprise a combination of springs, sliders and dashpots and are termed as contact constitutive models in DEM. With this simple contact modelling approach, the interactions between very large numbers of particles can be calculated with good computational efficiency which is the key fundamental aspect of DEM. The level of realism of the contact models can be advanced by specifying non-linear force-displacement relationships for the contact springs, or combining systems of springs and dashpots in various ways.

\subsubsection{Rheological model}

The approach of rheological or phenomenological models is firstly applied in continuum mechanics where the relations between stresses and strains are described by constitutive models. While in the context of DEM, these models are used to relate a contact displacement $\delta$ to a contact force $F$. In this approach, constitutive models are consisted of several base 
models. The graphical representations and load-deformation responses of these base models are illustrated in Figure 2.4.

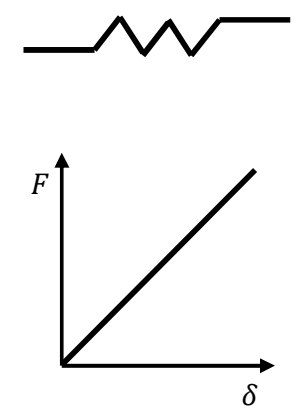

Linear elastic

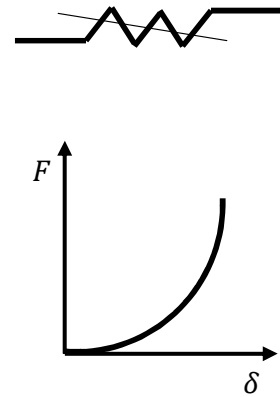

Non-linear elastic

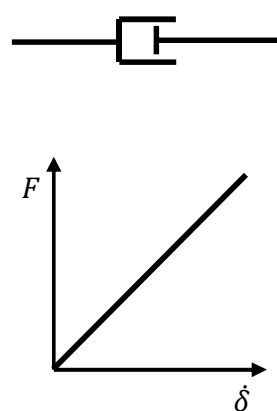

Ideal viscous

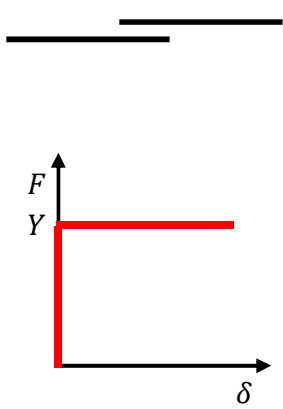

Rigid perfectly plastic

Figure 2.4: Graphical representation and load-deformation response of basic rheological models

As shown in Figure 2.4, a linear spring is used to represent the linear elastic model in which the load-deformation response is a straight line. For the non-linear elastic model, the response is a non-linear function. Both models are elastic and will not dissipate energy or capture plasticity in the response leading to the loading and unloading paths coincide. For the viscous response related to the deformation velocity, the representative model is a dashpot with damping $\eta$. The rigid perfectly plastic response is represented by a slider which activates at the yield point, i.e. when the force is smaller than the yield point $Y$, no deformation will occurs, and after this point, deformation continues at a constant load. More complex response can be captured by combining these basic rheological models.

\subsubsection{Normal contact models}

Linear elastic contact model The linear elastic contact model is the simplest normal contact model used in DEM. Then the contact force $F_{n}$ is calculated as

$$
F_{n}=K_{n} \delta_{n}
$$

where $K_{n}$ is the contact stiffness in the normal direction with the units force/length and $\delta_{n}$ is the overlap at the contact point. The orientation of this force is along the line joining the centre of the two contacting particles. The spring used in this model is conceptually considered as a penalty spring of which the spring constants cannot be derived from the material properties of the solid particles. The value of the spring constant can be determined by adjusting the value used in the simulation to obtain the overall response of an assembly to be the same as the one from the laboratory experiment.

Hertzian contact model The Hertzian contact model can overcome the non-physical nature of the linear spring stiffness. The normal contact force with non-linear contact stiffness 
is calculated as

$$
F_{n}=\left(\frac{2 G \sqrt{2 R}}{3(1-\nu)}\right) \delta_{n}^{\frac{3}{2}}
$$

where $G$ is the elastic shear modulus, $\nu$ is the Poisson's ratio, and $R$ is the sphere radius.

Walton-Braun linear model To consider the energy dissipation during particle collision, Walton and Braun [36] proposed a hysteretic linear contact model in which the loading and unloading paths are different. The normal force during first loading is given by

$$
F_{n}=K_{1, n} \delta_{n}
$$

while the normal force during unloading or reloading is given by

$$
F_{n}=K_{2, n}\left(\delta_{n}-\delta_{n, p}\right)
$$

where $\delta_{n, p}$ is the plastic deformation, which is depends on the maximum historical normal force, $F_{n, \max }$, i.e. $\delta_{n, p}=F_{n, \max } / K_{2, n}$. The stiffness during unloading is greater than during loading. Compared to the purely elastic model, one more parameter $F_{n, \max }$ or $\delta_{n, p}$ needs to be specified.

Spring-dashpot model In the spring-dashpot model [85], the energy dissipation at the contact point is considered by a dissipative viscous dashpot. The force-displacement relationship is given by

$$
F_{n}=K_{n} \delta_{n}+C_{n} \dot{\delta_{n}}
$$

where $C_{n}$ is the dissipative term which in Cleary [85]'s work can be calculated by

$$
C_{n}=2 \gamma \sqrt{m K_{n}}
$$

where the parameter $\gamma$ is a function of the coefficient of restitution $e$ as

$$
\gamma=-\frac{\ln (e)}{\sqrt{\pi^{2}+\ln (e)^{2}}}
$$

\subsubsection{Tangential contact models}

Though it is assumed that the surfaces of spherical particles are completely smooth which means no frictional resistance should exist at the contact point, a sliding friction parameter is included in the DEM simulation to consider the frictional resistance arose from the interlocking of asperities on the rough surfaces of the particles.

The tangential contact can describe the behaviour of contacting particles before and after sliding. The Coulomb friction model is used to define the initiation of the sliding. When $\left|F_{t}\right|<\mu F_{n}$ the contact sticks, but when $\left|F_{t}\right|=\mu F_{n}$ the sliding occurs, where $\mu$ is the coefficient 
of friction. As long as the contact remains stuck, the contact force is the product of the cumulative displacement in the tangential direction and the tangential spring stiffness. The cumulative displacement is the sum of the incremental relative displacement of the two particles at the contact point that occurs over each time increment from the time the contact is formed. For a cohesionless contact, the tangential force is given as

$$
F_{t}=-\min \left(\left|\mu F_{n}\right|, F_{t}\left(\delta_{t}, \dot{\delta}_{t}\right)\right) \frac{\dot{\delta}_{t}}{\left|\delta_{t}\right|}
$$

where $F_{t}\left(\delta_{t}, \dot{\delta}_{t}\right)$ is the pre-sliding shear force calculated using the contact constitutive model, $\delta_{t}$ is the cumulative relative deformation, $\dot{\delta}_{t}$ is the relative velocity which is a result of both relative translation of the particle centroids and rotation of the particles.

Linear tangential model Like the linear contact model for normal contact force, the basic contact model in the tangential direction also assumes a linear relationship between the tangential force and the cumulative tangential displacement. For the linear spring with stiffness $K_{t}$, the pre-sliding shear force at time is given as

$$
F_{t}\left(\delta_{t}, \dot{\delta}_{t}\right)=K_{t} \int_{t_{c}^{0}}^{t} \dot{\delta}_{t} d t
$$

where $t_{c}^{0}$ is the time at which the two particles initially contact. This integral is approximated by a summation in DEM as $\int_{t_{c}^{0}}^{t} \dot{\delta}_{t} d t \approx \sum_{t_{c}^{0}}^{t} \dot{\delta}_{t} \Delta t$.

Mindlin-Deresiewicz tangential model Mindlin and Deresiewicz [86] indicated that the tangential stiffness should depend on the current normal load, the current tangential load, the load history and whether the tangential load is increasing. This path-dependent nature of the tangential force has been considered by implementing an analogous but simpler constitutive model into DEM simulations in the previous works [87, 88].

\subsubsection{Rolling resistance models}

One obvious shortcoming of DEM simulation of spherical or circular particles with smooth surface is the absence of consideration of rolling resistance between contacting particles which will have an significant influence on the bulk behaviour of the particulate packing [89]. Two types of rotation occur between two contacting particles, one is a relative angular motion about an axis parallel to their common tangent plane, the other rotates about an axis orthogonal to the contact plane and along the contact normal. Not the same as the moments provided by the parallel bonds that cause the cement between the particles, the rolling resistance arises from geometrical aspects of the granular material. 
Iwashita-Oda model Iwashita and Oda [90] firstly proposed a 2D rolling resistance model in which an additional rotational spring-dashpot-slider system is added in parallel with the normal contact spring. The moment transfered by this model when the particles rotate relative to each other is given as

$$
M_{r}=-K_{r} \theta_{r}-C_{r} \frac{d \theta_{r}}{d t}
$$

where $K_{r}$ is the stiffness of the rotational spring, $C_{r}$ is the rotational viscous damping and $\theta_{r}$ is the relative rotation of which the incremental relative rotation is the combination of the incremental particle rotations and the change in orientation of the contact normal over the current time increment. The limiting value of $M_{r}$ is given by $\eta F_{n}$, where $\eta$ is the rolling friction.

The rolling resistance calculated by this model needs to be added to the moment transmitted by the tangential contact force. Then the rotational dynamic equilibrium equation for particle $p$ becomes

$$
\mathbf{I}_{p}=\frac{d \boldsymbol{\omega}_{p}}{d t}=\sum_{c=1}^{N_{c t}} \mathbf{F}_{t}^{c} r_{p}+\sum_{c=1}^{N_{c m}} \mathbf{M}_{r}^{c}
$$

The three dimensional form of this model is proposed by Belheine [91] and implemented in the DEM code YADE.

Jiang model Jiang et al. [92, 93] extended the above model to consider the rotational resistance depending on the contact area and the surface roughness.

In Jiang's original work, both the normal force and the moment depend on the rotation at the contact point, and an asymmetrical distribution of the contact normal traction is modelled. The normal force $F_{n}$ is given by

$$
F_{n}=\int_{-B / 2}^{B / 2}\left[k_{n}\left(\delta_{n}+\theta z\right)+\nu_{n}\left(\dot{\delta_{n}}+\dot{\theta} z\right)\right] d z
$$

where the contact overlap $\delta_{n}$ is at the centre of the contact $(z=0), \theta$ is the rotation and $\nu_{n}$ is the viscous damping. Then contact overlap is taken to be positive in compression and counterclockwise rotation is positive. The value of $B$ depends on the grain shape.

The moment at the contact is caused by the contact normal traction given as

$$
M_{n}=-\int_{-B / 2}^{B / 2}\left[k_{n}\left(\delta_{n}+\theta z\right)+\nu_{n}\left(\dot{\delta_{n}}+\dot{\theta} z\right)\right] z d z
$$

In Jiang'g model, the width of the contact can be considered explicitly which represents the real physical situation better. Then Jiang et al. [93] improved this model by introducing a second parameter $N$ which gave the number of asperities distributed homogeneously across the contact area. Each asperity will itself form a separate contact and the total normal force and moment are the sum of all the asperities' contributions. 


\subsubsection{Bond models}

A bond can be introduced in DEM to model the tensile force caused by the cement between particles by specifying a tensile strength in the normal direction and a shear strength in the tangential direction.

Linear bond model In a simple linear tensile model, the bond strength is specified as $F_{n}^{\max }$ in units of force. The tensile-capable contact model is active when $\delta_{n}<-\delta_{n}^{t \text {,max }}$, and $\delta_{n}^{t, \max }=F_{n}^{t, \max } / K_{n}$. If the normal tensile strength is exceeded, then the contact is removed. This linear tensile model can be found in the previous works [94-96].

Parallel bond model Considering that the cement at the particle contacts always covers a finite area in reality, it is reasonable to develop the parallel bond model [39] in which the strength of the bond depends on the volume of the cement. Furthermore, a moment can be transmitted with this cemented bond with finite area which is another improvement compared to the linear bond model. In the parallel bond model, a pair of parallel linear springs are set at each cemented contact. The parallel bond has a finite area with the radius $R_{\text {bond }}=\alpha r_{\text {min }}$, where $\alpha$ is the parallel bond radius multiplier and $r_{\min }$ is the smaller radius of the two contacting particles. The bond area $A_{p b}$ is given by $A_{p b}=\pi R_{\text {bond }}$ in $2 \mathrm{D}$ and $A_{p b}=\pi R_{\text {bond }}^{2}$ in 3D. Unlike the linear bond model, the stiffness is specified in units of stress/displacement and the maximum strength is specified in units of stress.

The forces carried by the parallel bond in the normal and tangential directions are given by

$$
\begin{aligned}
& F_{p b}^{n}=K_{p b}^{n} A_{p b} \delta_{n} \\
& F_{p b}^{t}=K_{p b}^{t} A_{p b} \Delta \delta_{t}
\end{aligned}
$$

where $K_{p b}^{n}$ is the bond normal stiffness, $K_{p b}^{t}$ is the bond shear stiffness.

The moments transmitted by the parallel bond are the spin moment $M_{p b}^{\text {spin }}$ and the bending moment $M_{p b}^{\mathrm{b}}$. Then the spin moment only exists in the 3D case as it relates to a moment caused by relative rotation about the contact normal. The increments of these two moments are calculated by the incremental rotation of the particles as

$$
\begin{aligned}
& \Delta M_{p b}^{\mathrm{spin}}=K_{p b}^{t} I_{p b} \Delta \theta_{n} \\
& \Delta M_{p b}^{\mathrm{b}}=K_{p b}^{n} I_{p b} \Delta \theta_{s}
\end{aligned}
$$

where $I_{p b}$ is the moment of inertia of the parallel bond, $\Delta \theta_{n}$ is the incremental rotation about the contact normal, and $\Delta \theta_{s}$ is the incremental rotation orthogonal to the contact normal. The contact forces will add additional contributions to the moment given by the cross product of the resultant contact force.

Then the tensile stress $\sigma$ and shear stress $\tau$ caused by the forces and moments can be derived 
by considering the beam bending theory

$$
\begin{aligned}
\sigma & =\frac{-F_{p b}^{n}}{A_{p b}}+\frac{\left|M_{p b}^{\mathrm{b}}\right|}{I_{p b}} R_{\text {bond }} \\
\tau & =\frac{\left|F_{p b}^{s}\right|}{A_{p b}} R_{\text {bond }}+\frac{\left|M_{p b}^{\text {spin }}\right|}{J_{p b}} R_{\text {bond }}
\end{aligned}
$$

where $J_{p b}$ is the polar moment of inertia of the parallel bond in 3D.

The bond normal strength is $\sigma_{n}^{\max }$ and the bond shear strength is $\tau^{\max }$. The bond will be removed once the normal stress or shear stress exceeds the corresponding strengths. Related studies can be found in [97-100].

\subsection{Dynamic solutions}

The particles in granular materials are analogous to the degrees of freedom in a matrix structural analysis (the end points of the structural elements) or the nodes in a finite element mesh. Therefore, the overall governing equation for the granular system can be expressed as the standard governing equation for a dynamic analysis in structures or continuum finite element given by

$$
\mathbf{M u}+\mathbf{C u}+\mathbf{K u}=\mathbf{F}
$$

where $\mathbf{M}$ is the mass matrix, $\mathbf{C}$ is the damping matrix, $\mathbf{u}$ is the incremental displacement vector and $\mathbf{F}$ is the incremental force vector. The terms in Equation 2.21 have the following meaning: $\mathbf{M} \ddot{\mathbf{u}}$ is the inertia force, $\mathbf{C} \dot{\mathbf{u}}$ is the damping force, $\mathbf{K u}$ is the internal force and $\mathbf{F}$ is the external force. The global stiffness matrix $\mathbf{K}$ depends on the system geometry, i.e. which particles are contacting.

Both implicit and explicit approaches can be used to solve the dynamic equilibrium equation for a multi-nodal system. In the implicit approach, the vector $\mathbf{u}$ and $\mathbf{F}$ are constructed for all the particles. The global mass $\mathbf{M}$, stiffness $\mathbf{K}$ and damping $\mathbf{C}$ matrices are combined as for the finite element method [101] Then a large system of simultaneous equation is generated when solving the dynamic equilibrium equation involving the assembly of a stiffness matrix. The solution will involve inversion of a highly sparse stiffness matrix and the sequence of calculations will be very computationally expensive both in terms of the number of operations required to solve the system and in terms of memory requirements.

Therefore, an explicit approach is used in DEM simulation in which the solution of the global system is avoided by considering the dynamic equilibrium of the individual particles rather than solving the entire system simultaneously. 


\subsubsection{Governing equations}

The equation governing the translational dynamic equilibrium of particle $p$ is given by

$$
m_{p} \ddot{\mathbf{u}}_{p}=\sum_{c=1}^{N_{c, p}} \mathbf{F}_{p c}^{\mathrm{con}}+\sum_{j=1}^{N_{n c, p}} \mathbf{F}_{p j}^{\mathrm{non}-\mathrm{con}}+\mathbf{F}_{p}^{\mathrm{f}}+\mathbf{F}_{p}^{\mathrm{g}}+\mathbf{F}_{p}^{\mathrm{app}}
$$

where $\ddot{\mathbf{u}}_{p}$ is the acceleration vector, $\sum_{c=1}^{N_{c, p}} \mathbf{F}_{p c}^{\mathrm{con}}$ are the contact forces due to contact $c$ when there are $N_{c, p}$ contacts between particle $p$ and either other particles or boundaries, $\sum_{j=1}^{N_{n c, p}} \mathbf{F}_{p j}^{\text {non-con }}$ are non-contact forces between particle $p$ and $N_{n c, p}$ other particles or boundaries, $\mathbf{F}_{p}^{\mathrm{f}}$ is the fluid interaction force, $\mathbf{F}_{p}^{\mathrm{g}}$ is the gravitational force and $\mathbf{F}_{p}^{\mathrm{app}}$ is a specified applied force.

The dynamic rotational equilibrium is given by

$$
\mathbf{I}_{p} \frac{d \boldsymbol{\omega}_{p}}{d t}=\sum_{j=1}^{N_{\mathrm{mom}}} \mathbf{M}_{p j}
$$

where $\boldsymbol{\omega}_{p}$ is the angular velocity vector and $\mathbf{M}_{p j}$ is the moment applied by the $j$ th moment transmitting contact forces involving particle $p$ and there are a total of $N_{\text {mom }}$ moment transmitting contacts.

Then the particle translational and rotational accelerations $\ddot{\mathbf{u}}_{p}$ and $\dot{\boldsymbol{\omega}}_{p}$ can be calculated from the above two govern equations. These acceleration values can be used to obtain incremental displacements and hence update the particle positions.

\subsubsection{Time integration}

In numerical analysis, the techniques used to update parameters given their first and second derivatives with respect to time are called time integration methods. Then the centraldifference method with a time increment $\Delta t$ is used in DEM simulation.

The velocity at time $t+\Delta t / 2$ is calculated as

$$
\mathbf{v}_{p}^{t+\Delta t / 2}=\mathbf{v}_{p}^{t-\Delta t / 2}+\mathbf{a}_{p}^{t} \Delta t
$$

where $\mathbf{v}_{p}^{t-\Delta t / 2}$ and $\mathbf{v}_{p}^{t+\Delta t / 2}$ are the velocity vectors at $t-\Delta t / 2$ and $t+\Delta t / 2$ and $\mathbf{a}_{p}^{t}=\ddot{\mathbf{u}}_{p}$ is the acceleration vector at time $t$.

The velocity at time $t+\Delta t / 2$ is taken to equal the average velocity over the increment from $t$ to $t+\Delta t$. Then the particle position is updated as

$$
\mathbf{x}_{p}^{t+\Delta t}=\mathbf{x}_{p}^{t}+\mathbf{v}_{p}^{t+\Delta t / 2} \Delta t
$$




\subsubsection{Accuracy and stability}

This explicit central-difference time integration scheme in DEM is computationally efficient but with the limitation that this scheme is only conditionally stable. The incremental changes in the particle positions and contact forces in a time step must be small to capture the inherent non-linearity of the problem (changing in contact structure and non-linear contact response). Cundall and Strack [34] stated that a fundamental idea of DEM is that the time step is chosen to be sufficiently small that in a single step disturbances cannot propagate from a disk further than its neighbours. It means that the motion of a particle over a given time step only influences its immediate neighbouring particle.

In the context of modelling the physical system, it is important to consider the accuracy, stability and robustness of the numerical method and fully understand the limitations and approximations involved. The system of differential equations is an idealisation of the real particle assembly, limiting accurate prediction. The approximation errors are introduced when the equation is solved numerically. The round-off error is introduced by the calculations using computers. The second error is the truncation error introduced as a consequence of the approximations when calculating the incremental displacements from the calculated accelerations. The truncation error exists in any numerical model at each time step that simulates the response of a transient or dynamic system. The truncation error can be understood by reference to the Taylor series expansion. The term $O\left(\Delta t^{n+1}\right)$ is the truncation error and is proportional to $t^{n+1}$. The central difference algorithm used in DEM is a second order scheme, i.e. the accuracy of the calculated displacement depends on the square of the time increment, $\Delta t^{2}$.

There are several ways to explain the stability of the numerical modelling. If there is a linear growth relationship between the global error and the local error, then the algorithm is typically stable. Otherwise, the total energy including the strain energy stored in the contact springs and the particles' kinetic energy of the system is monitored in mechanics applications analysts. When the numerical integration is stable there will be no drift in the energy of the system.

The stability of DEM can be considered in context of the free vibration of a particle of mass, $m$, suspended on a simple, elastic spring with stiffness $k$. For this single degree of freedom system, the dynamic equilibrium equation is given by $a=-k x$, where $a=\ddot{x}$. If the central difference approach is used to this system, the maximum time increment can be used is $\Delta t=T / \pi$, where $T$ is the period for free oscillation of system. This period is calculated as $T=2 \pi \sqrt{m / k}$. The restriction of the time increment for this simple, single degree of freedom system also applies to the multi degrees of freedom simulations in DEM [102].

\subsection{Packing evaluation method}

The particle packing plays an important role in leading the physical behaviour of the granular system. As the topology of the system is highly complex, it is difficult to observe the way 
particles packed around each other by experiments. With the development of DEM, more detailed information on the internal structure of particulate systems can be accessed.

Currently, the techniques applied to investigate the features of particle packings focus on the packing density, orientations of the particle contacts, and internal (topological) structures of packings. The classical characteristics such as particle size distribution and packing density cannot consider the spatial distribution of a packing. Besides, the method involves the use of a radial function is less effective [103]. When a packing is subject to external loading and generates contacts between particles, more features can be checked such as the coordination number, contact force distribution and contact orientation distribution [104]. These techniques may be classified into different categories from different perspectives. From the perspective of problem scale, they are macroscopic based (stress, strain and critical void ratio [105]) and microscopic based (coordination number, stress tensor and fabric tensor [45]). While from the perspective of the specific information to be extracted from the particle system, the methods can be classified into two categories: one is focus on the void ratio $[32,106]$ of the packing and the other pays attention on the contact network [107].

\subsubsection{Packing density}

The most commonly used characterisation of a packing is its dense condition which is described by different parameters.

The void ratio $e$ is given by

$$
e=\frac{V_{v}}{V_{s}}
$$

where $V_{v}$ is the volume of voids and $V_{s}$ is the volume of solid particles.

The specific volume $v$ is the total volume occupied by the material per unit solid volume given as

$$
v=\frac{V}{V_{s}}
$$

The porosity $n$ is defined as the ratio of the volume of voids to the total volume

$$
n=\frac{V_{v}}{V}
$$

and the solid volume fraction is defined as

$$
\nu=\frac{V_{s}}{V}
$$

It should be noted that the range of void ratio values differs for $2 \mathrm{D}$ and $3 \mathrm{D}$ systems. For example, the densest packing configuration for uniform disks (hexagonal packing) has a density of 0.906 in the $2 \mathrm{D}$ case while for uniform spheres in $3 \mathrm{D}$ with hexagonal close packing (HCP) or face centred cubic packing, the largest density that can be obtained is 0.740 . On the other hand, as the density parameters are only scalar measurements, it cannot illustrate the 
inhomogeneity of the density distribution in the packing. One feature highlighted by Marketos and Bolton [108] is that the packing density is influenced by the boundary condition and a local decrease can be observed close to the boundary.

Another observation [109] is that additional heterogeneity will be introduced when a shear band forms. Furthermore, the measured density of the granular material will be influenced by the size of the representative volume element (RVE) [80]. Therefore, care must be taken to use a RVE which is sufficient large to the proper density which can representative the granular material statistically. DEM simulations also provide a feasibility to define alternative density parameters. Kuhn [110] proposed an effective void ratio including only those particles that participate in stress transmission when calculating the volume of solids.

\subsubsection{Radial distribution function}

The radial distribution function $g(r)$, also called the pair correlation function, is the classical summary characteristic of spatial variability for statistically homogeneous systems of sphere centres. Consider a system of $N$ particles in a volume $V$. If a given particle is taken to be at the origin $O$, and if $\rho_{n}=N / V$ is the average number density of particles, then the local time-averaged density at a distance $r$ from $O$ is $\rho_{n} g(r)$.

The radial distribution function is a measure of the probability of finding a particle at a distance of $r$ away from a given reference particle. It needs to determine how many particles are within a distance of $r$ and $r+d r$ away from a particle.

\subsubsection{Coordination number}

It is obvious that the density parameters can not consider the particulate structure of the particle packing. The coordination number can measure the intensity at the particle scale with the definition of the number of contacts per particle

$$
Z=2 \frac{N_{c}}{N_{p}}
$$

where $N_{c}$ is the total number of contacts and $N_{p}$ is the number of particles. The multiplier is used because each contact is shared between two particles.

Further work has been done to improve or refine the definition of the coordination number. Thornton [111] defined a mechanical coordination number $Z_{m}$ as

$$
Z_{m}=2 \frac{N_{c}-N_{p}^{1}}{N_{p}-\left(N_{p}^{1}+N_{p}^{0}\right)}
$$

where $N_{p}^{0}$ and $N_{p}^{1}$ is the numbers of particles with zero contact or only one contact which cannot participate in transmitting stress and are termed as floaters.

Another effective coordination number $Z_{p}$ was proposed by Kuhn [110] with a higher level 
of discrimination in selecting particles which only considered the particles participate in the load-bearing framework.

Some complementary concepts have been developed to futher understand the coordination number. The granular material can be analysed from the perspective of network analysis of which the particle is regarded as a node and the contact is a connection. Then the degree of a particle [112] is the number of the contacts related to it. The degree distribution $P_{c}(k)$ defines the probability that a given particle will have $k$ contacts. The average degree equals the coordination number, i.e.

$$
Z=\sum_{k=1}^{\infty} k P_{c}(k)
$$

Some useful conclusions can be obtained based on this concept such as smaller particles are less likely to be participating in the strong force chains in comparison with larger particles. The heterogeneity of the packing can be assessed by the standard deviation of the coordination numbers [113].

Another concept is the caged particle [114] of which the movement is curtailed by neighbouring particles. The caging number is defined as the minimum average number of contacts required to immobilize a particle.

Alternative contact indices have also been proposed to quantify the contact intensity [115]. For example, the contact index parameter $C I[116]$ is defined as

$$
C I=\frac{1}{N_{p}} \sum_{i=1}^{N_{p}} \frac{1}{S_{p_{i}}} \sum_{j=1}^{N_{c, i}} S_{c_{j}}
$$

where $N_{p}$ is the total number of particles considered, $S_{p_{i}}$ is the surface area of particle $i, S_{c_{j}}$ is the surface area of contact $j$ and $N_{c, i}$ is the number of contacts involving particle $i$.

Redundancy The concept of redundancy in civil engineering can also be applied to granular mechanics. Then the stability of the granular system is related to the coordination number [117]. For the frictionless system, the static equilibrium can be achieved when $Z \geq 4$, while for the frictional system where shear contact forces can impart moments, the requirement for the stability is $Z \geq 3$.

In the $2 \mathrm{D}$ case, the redundancy factor $R$ of the system is defined by the ratio of the number of degrees of freedom at the contact points divided by the number of the governing equations

$$
R=\frac{2 N_{c}^{\mathrm{el}}+N_{c}^{\mathrm{fr}}}{3\left(N_{p}-N_{p}^{0}\right)}
$$

where $N_{c}^{\mathrm{el}}$ is the number of elastic contacts (where the shear force does not exceed the shear resistance given by the Coulomb friction), $N_{c}^{\mathrm{fr}}$ is the number of frictional contacts. 


\subsubsection{Contact force distribution}

The coordination number considers the contacts in the granular packing, while it still cannot illustrate the magnitudes of the contact force. To overcome this shortcoming, the analysis method based on the probability distribution of the magnitude of the contacts has been proposed. The probability of a contact transmitting a given force is plotted against the value of the force. The probability distribution function can be obtained by fitting these data. The observation that the simulation results can be well fitted by an exponential function for forces that extended the average force can be found in many studies [118-120]. For the tangential component of the contact forces, conclusions have been drawn that particle sliding tends to occur outside the strong force chains and the proportion of sliding contacts remains essentially constant after an initial increase during the shear process.

\subsubsection{Fabric}

The density and coordination number are scalar measures of a packing which can not describe the anisotropy of the granular material. The term fabric or structure refers to the arrangement of particles, particle group and pore spaces in the granular material. Both experiment and simulation results show that the anisotropy has a great influence on the bulk behaviour of the granular material. The anisotropy can be classified into inherent, induced and initial [121] of which the inherent anisotropy is caused by the depositional process [122], the induced anisotropy is related to strain and stress, and the initial anisotropy is both related to deposition and the geological stress history. Different axes are chosen to quantify the anisotropy, including the orientations of the particles, the orientations of the vectors linking the centroids of contacting particles, or the orientations of the contact normals. Proper methods have been developed to interpret the orientation data to provide a meaningful measure of any preferred orientation of the vectors. The two commonly used approaches are the fitting of curves to rose diagrams or the fabric tensor approach. The anisotropy quantified will be a measure of the frequency of particles being oriented in the most preferential orientation relative to the frequency of particles having the least preferential orientation [102].

Histograms and curve fitting The polar histogram or rose diagram is created to show the number of contacts oriented with the predefined angular interval. The analysis of them is achieved by applying an analytical function to fit the histogram and using the parameters of the function to quantify the intensity of the anisotropy and the preferred orientation. The basic idea is that the orientations can be described using a probability density function (PDF) $E(\mathbf{n})$ (also called fabric ellipsoid by Oda et al. [113]) which indicates the likelihood that a contact will have the orientation of unit vector $\mathbf{n}$. The integral of this function over the domain should be 1 .

$$
\int_{\Omega} E(\mathbf{n}) d \Omega=1
$$


where $d \Omega$ is the differential solid angle in a spherical coordinate system. This fabric ellipsoid will have a spherical shape for the isotropic material. Otherwise, the ellipsoid long-axis orientation gives the preferential orientation of the contact normals. It is also stated that the fabric ellipsoid is the second most important index describing the structure of a granular material after the void ratio [113].

To analyse the $2 \mathrm{D}$ data obtained from $2 \mathrm{D}$ systems or axisymmetric systems in $3 \mathrm{D}$, the fitting function can be expressed as

$$
\int_{0}^{2 \pi} E(\theta) d \theta=1
$$

where $\theta$ is the inclination to the reference axis. This function can be expanded by different Fourier series [123]. Rothenburg and Bathurst [124] used a Fourier series expansion with two terms given as

$$
E(\theta)=\frac{1}{2 \pi}\left[1+a \cos 2\left(\theta-\theta_{a}\right)\right]
$$

where $a$ is a parameter defining the magnitude of fabric anisotropy and $\theta_{a}$ defines the direction of the principal fabric. Furthermore, a quantitative link has been established between the fabric parameters and the macro-scale stress-strain response.

Fabric tensor The fabric tensor which can be obtained from the data of orientation vectors is another measure to determine the preferred orientation and the magnitude of the anisotropy. The most commonly used definition of the fabric tensor is the second-order fabric tensor given as $[125,126]$

$$
\Phi_{i j}=\frac{1}{N_{c}} \sum_{k=1}^{N_{c}} n_{i}^{k} n_{j}^{k}
$$

where $n_{i}^{k}$ is the unit vector describing the contact normal orientation.

The fabric tensor is an abstract concept and can be analysed in the way analogous to the interpretation of the stress tensor considering their similarities of being second-order and symmetric. Therefore, the preferred orientations and the magnitude of the anisotropy can be calculated when the fabric tensor is known. The magnitude of the major fabric is given by $\Phi_{1}$, the minor fabric is given by $\Phi_{3}$, and the intermediate fabric is $\Phi_{2}$ in three dimensions. The principal fabric parameters can be determined by the eigenvalue decomposition of the fabric tensor. The extent of the bias in the most preferential direction of fabric orientation is given by the largest eigenvalue and the corresponding eigenvector gives the direction of the principal fabric component [102]. The principal fabric components can also be obtained from the expressions similar to the one used in continuum mechanics [127] when calculating principal stresses.

For a 2D or axisymmetric system, the principal fabric components are given as

$$
\left(\begin{array}{c}
\Phi_{1} \\
\Phi_{3}
\end{array}\right)=\frac{1}{2}\left(\Phi_{x x}+\Phi_{y y}\right) \pm \frac{1}{2} \sqrt{\left(\Phi_{x x}-\Phi_{y y}\right)^{2}+\Phi_{x y}^{2}}
$$


Then the intensity of anisotropy of the system can be quantified by using the fabric components either by considering the difference $\Phi_{1}-\Phi_{3}$ [111] or the ratio $\Phi_{1} / \Phi_{3}$ [128].

For the 3D case, Barreto et al. [129] proposed quantification of three-dimensional deviator fabric using the following invariant (analogous to the shear stress in the octahedral plane)

$$
\Phi_{d}=\frac{1}{\sqrt{2}} \sqrt{\left(\Phi_{1}-\Phi_{2}\right)^{2}+\left(\Phi_{2}-\Phi_{3}\right)^{2}+\left(\Phi_{3}-\Phi_{1}\right)^{2}}
$$

In Woodcock's work [130], two parameters $\beta_{1}$ and $\beta_{2}$ are considered

$$
\begin{aligned}
& \beta_{1}=\ln \left(\frac{\Phi_{1}}{\Phi_{3}}\right) \\
& \beta_{2}=\ln \left(\frac{\Phi_{1}}{\Phi_{3}}\right) / \ln \left(\frac{\Phi_{2}}{\Phi_{3}}\right)
\end{aligned}
$$

The value of $\beta_{1}$ indicates the extent of the concentration of vectors in the preferred orientation, and the $\beta_{2}$ value determines whether the distribution of orientations is a cluster or a girdle.

Some previous works are devoted to understanding the relationship between the fabric tensor with the macro-scale material behaviour. The best correlation can be found between the macro-scale response (the principal stress ratio $\sigma_{1} / \sigma_{3}$ ) and the ratio of the major to minor fabric for the contact normal orientations [131, 132]. This phenomena can be easily explained that the expression for the fabric tensor $\left(\Phi_{i j}=\frac{1}{N_{c}} \sum_{k=1}^{N_{c}} n_{i}^{k} n_{j}^{k}\right)$ is very similar to the expression for the stress tensor $\left(\sigma_{i j}=\frac{1}{V} \sum_{k=1}^{N_{c}} f_{i}^{k} l_{j}^{k}\right)$. The evolution of the principal fabric orientation is tied in with the development of strong force chains whose alignment develops during loading so that they can transmit the applied stresses [113].

\subsection{Multiscale modelling}

The high computational cost of DEM models restricts its analysis with granular materials realistically in term of the actual particle numbers and geometries. The simulation of a large number of particles requires unacceptable computational time that is the most critical problem existing in the industrial application of DEM. The real industrial applications require billions of particles (macro-scale problem) compared with the calculating capability of several million particles (micro-scale problem) on a single personal computer. Tackling this problem is a key ongoing challenge involved in DEM analysis. Though with the rapid development of the computer hardware that DEM codes can run in a parallel or high performance computing environment [133, 134], methodological developments and improvements of DEM are still needed to handle the practical large scale problem in research and industry. Therefore, multiscale modelling of granular materials has received an increasing attention in recent years [135] as these approaches allow to naturally embed a refined description of the complexity of the material into a full structural engineering problem. These multiscale strategies can be classified into two categories: coarse graining and combined finite-discrete element method 
(FEM-DEM).

It is worth noting that considering materials on multiple scales is a relative concept for the terms of macro-scale, meso-scale and micro-scale. Based on the above description, in granular materials, the micro scale is taken to be a scale where individual particle responses are measurable, i.e. the contact forces and particle displacements can be distinguished. However, if particle damage is considered, the relevant micro-scale is the sub-particle scale where the particles are regarded as a continuum to consider the stress and strain distributions.

\subsubsection{Coarse graining}

The coarse graining technique is a possible approach to tackle the macro scale problem. To put it simply, when using coarse graining in DEM the particles are artificially enlarged in the model which significantly reduces the number of particles in the system therefore the calculation time is acceptable. It is obvious that when use large-sized particles in DEM simulation the performance such as the energy dissipation of the coarse-graining system is not same with the original system. Therefore, when the DEM simulation is performed by using such large-sized particles, some theoretical issues need to be addressed to explain the difference between the original system and the coarse graining system. Moreover, a systemic framework needs to be proposed to develop a reliable coarse graining system which can represent the original system well.

Such a concept based on an intuitive thought that a group of small particles could be represented by a large particle was first suggested by Kazari [136]. The approach based on this concept has been the most commonly used method for the coarse graining problem. The later improved models are called the similarity model [137], the imaginary sphere model [138], the similar particle assembly model [139] and the coarse grain model respectively [140]. These models are proposed to target some specific applications with several assumptions valid only in the corresponding situations. Mokhtar [141] proposed the similar particle assembly (SPA) model for the fluidization of Geldart's group A and D particles. Sakai developed the coarse grain model in 2009 which then had been applied to the simulation of a three-dimensional plug flow in horizontal pipeline [140], a two-dimensional bubbling fluidized bed [142], and a fluidized bed with fine particles where the coarse grain model was improved by considering the cohesive force. Baran [143] conducted the simulations for efficient packed-bed and industrial size fluidized bed with such coarse grain model. Hilton [144] investigated the effectiveness of the coarse grain approach for gas flow through particle beds using resolved and coarse grain models with the same effective particle numbers. Some analogous research can be found in the work of $\mathrm{Lu}$ [145], Chu [146] and Elghannay [147].

\subsubsection{Combined FEM-DEM}

Generally speaking, the finite element method is suitable to solve the large scale problem while the application to complex granular materials may be unsatisfactory because of the lack 
of an adequate mechanical constitutive law. One the other hand, the discrete element method is able to capture the nature of the granular material but with the restriction to be applied to a full-engineering problem. The combined FDEM method allows to efficiently overcome the common drawbacks of both finite element method and discrete element method. The two methods have been coupled in the framework of a multiscale numerical homogenization approach to exploit the efficiency of FEM at solving boundary value problems at the structural level and the capability of DEM to capture complex material behaviours.

Several multiscale modelling schemes have been proposed. Kaneko et al. [148] employed the mathematical homogenisation theory for the construction of a multiscale modelling approach. Miehe et al. [149] proposed an original scheme for quasi-static homogenisation of granular microstructures and its embedding into a two-scale modeling framework. In these methods, the DEM is used to derive an equivalent mechanical law for the microstructure behaviour. Andrade et al. [150] and Avial et al. [151] developed a discrete continuum approach based on a numerical homogenisation scheme in which friction and dilatation at the microstructure are transferred to the elasto-plasticity continuum at the macroscale level. Nitka et al. [152], Nguyen et al. [153], Guo and Zhao [154], and Desrues et al. [155] applied the concept of the representative volume element (RVE) to build a fully coupled multiscale FEM-DEM approach based on computational homogenisation. The stress state at the macroscale level is obtained for each Gauss point from the associated DEM granular assembly (RVE).

The typical coupling steps involved in FEM-DEM can be described as follows. As in the standard finite element method, the displacement gradient increment is applied at the Gauss point level to obtain the corresponding stress state. In the FEM-DEM scheme, the displacement gradient increment acts as an updated boundary condition on the discrete element REV at the Gauss point level. The subsequent discrete element numerical simulation acts as a material constitutive relation by returning the new stress state at the macro level. Therefore, the homogenisation theory is a key point which link the granular and continuum scales.

Representative Volume Element (RVE) The RVE is defined to be a volume than can statistically represent the material under consideration [156]. Recognising the inherent inhomogeneity in a granular material because of the particles and contact force orientations and magnitudes, the scale at which it is considered to be a continuum must be significantly larger than the particles themselves. An increase in size will not change the measured parameter.

The appropriate diameter $D_{\mathrm{RVE}}$ of the RVE is suggested to be as $D_{\mathrm{RVE}}>10^{3}$. While in DEM simulations to date the ratios used are often much smaller than this suggested limit. There are times when, while the use of an RVE may not be valid, continuum terminology is still relevant. The use of a RVE is equally applicable when quantifying the packing arrangement of particles.

Homogenisation The methods to translate particulate mechanics into continuum mechanics are called homogenisation techniques which are the averaging procedures to calculate the 
representative stresses and strains from the discrete forces and displacements calculated in DEM. The homogenisation or averaging techniques used in DEM can be classified to be volume, time-volume, or weighted time-volume methods. The commonly used volume averaging methods are applicable to quasi-static systems where the inertia effects are ignored. In general, an average or representative value for the quantity or parameter should first be assigned to each particle [157]. Then the average value for the parameter $Q$ is given by

$$
Q=\langle Q\rangle=\frac{1}{V} \sum_{p \in V} w_{v}^{p} V^{p} Q^{p}
$$

where $Q^{p}$ is the representative value of the parameter for particle $p, V^{p}$ is the volume of particle $p$, and $w_{v}^{p}$ is the weighting assigned to particle $p$.

\subsection{Concluding remarks}

In this Chapter, the basic of DEM has been introduced briefly including the governing equation, the time integration method and the accuracy and stability of this algorithm. The theories related to the three aspects of this thesis have been presented in detail together with the corresponding literature reviews. For the contact problem between particles, the common used particle shapes and some contact models are introduced in Section 2.2 and Section 2.4. For the packing evaluation strategies, the conventional characterising methods of particulate system are described in Section 2.6. The two strategies of coarse graining methods and combined FEM-DEM methods which are used to treat the multiscale problem are introduced in Section 2.7. 


\section{Part II}

Contact models for rough particles 


\section{Chapter 3}

\section{Surface roughness modelling $-\mathbf{I}$. Classical GW model}

\subsection{Introduction}

From the perspective of the geometric representation in the DEM, the most commonly used primitive geometric entities are disks and spheres, with ellipses and ellipsoids used to a much lesser extent, but all assumed to have smooth surfaces. However, real particles contain geometric irregularities or randomness at both microscopic and macroscopic levels. By recognising the significant influence of particle shapes on the mechanical behaviour of particle systems, the current discrete element modelling of irregularities of real particles has mostly been focused on the macroscopic level. In addition to the introduction of nonspherical entities such as polygons, polyhedra, super-quadrics, cylinders etc. [78, 158], more complicated geometric shapes are often represented by bonding or clumping together several basic entities [159-163]. Nevertheless, surface irregularities at the microscopic level, also called the surface roughness, are more difficult to be accounted for, although they may have strong influence on the phenomena of contact, friction, wear and lubrication [164].

The contact laws mostly employed in the DEM, such as the linear contact model and the Hertzian contact model, are intended for contact between smooth particles. It is therefore necessary to quantitatively improve the classical DEM by taking the surface roughness into consideration.

Several approaches have been developed to understand the contact mechanism between rough surfaces. Two key issues need to be addressed: the mathematical description of rough surfaces, and the modelling of microscopic contact mechanisms. The modelling of rough surfaces can be classified into two categories: statistical and deterministic. The earliest and most recognized statistical treatment of rough surfaces is the Greenwood and Williamson (GW) model [64], in which a rough surface is described as an assembly of asperities whose properties are obtained from a given statistical height distribution, and then the Hertzian contact solution is applied to 
each asperity to obtain an overall contact pressure distribution. For the contact between two rough curved bodies instead of two nominally flat surfaces, the first analytical investigation is conducted by Greenwood and Tripp [165] who employ the GW asperity contact model together with the bulk surface deformation for circular point contact.

The GW model can be viewed as a single scale method since the statistical parameters used to represent rough surfaces are scale-dependent. An early attempt of using multiscale methods is made by Archard [166] who models the asperities of rough surface as protuberance upon protuberance. Another statistical approach is introduced by Majumdar and Bhushan [167], where a fractal curve/surface is adopted to describe a rough surface, together with a contact mechanism to resolve the contact. This fractal based approach can be regarded as multiple scaled because of the inherent multi-scale invariant characteristics of the most fractal curves/surfaces.

On the other hand, the deterministic methods attempt to model rough surfaces in a definitive manner and the resulting contact problem is typically solved by the finite element method. Furthermore, the fast Fourier transformation can also be used to represent rough surfaces [168]. Considering the simplicity and popularity of the GW model, a new normal interaction law is established for spherical particles with random rough surfaces.

\subsection{The classic GW model}

A rough surface consists of a myriad of asperities or peaks that restrict the real contact area when two such surfaces are in contact. Due to the complexity of a rough surface, an appropriate mathematical expression is needed to model a real surface as a profile with a particular statistical distribution of asperities, for instance, the Gaussian or the exponential distribution. This statistical approach to mathematically represent rough surfaces is adopted in the GW model [64]. By further combining with the Hertzian elastic theory, a solution to the contact problem of rough surfaces is derived.

Several assumptions are made in the classic GW model: 1) The height profile of a rough surface is assumed to obey a Gaussian distribution; 2) The summits of the asperities are spherical with constant curvature; 3) Each individual asperity deforms separately; and 4) The bulk surface deformation below the individual asperity is negligible.

\subsubsection{Characteristics of rough surfaces}

The characteristics of a rough surface are obtained on the basis of the profile which is the line of a cross section in a direction perpendicular to the surface as shown in Figure 3.1. From this profile, surface roughness parameters are determined by scrutinising a set of points $z\left(x_{i}\right)(i=1, \cdots, N)$ which give the heights from the mean line in the sample length interval $L$. The main surface roughness parameters include: 


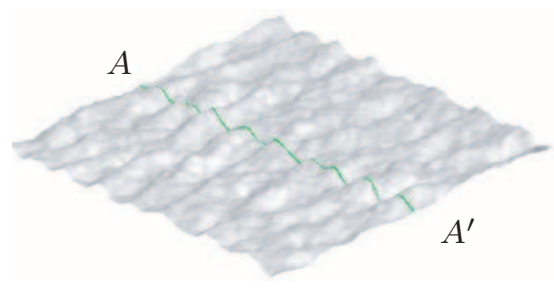

(a) $3 \mathrm{D}$ rough surface

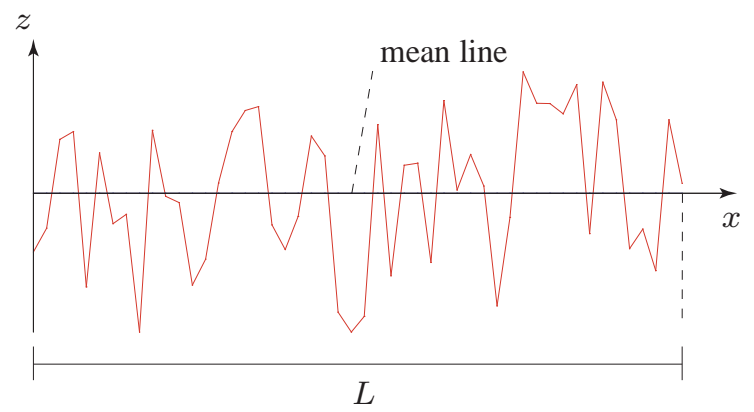

(b) Profile of the cross section $A-A^{\prime}$

Figure 3.1: Topography of a rough surface

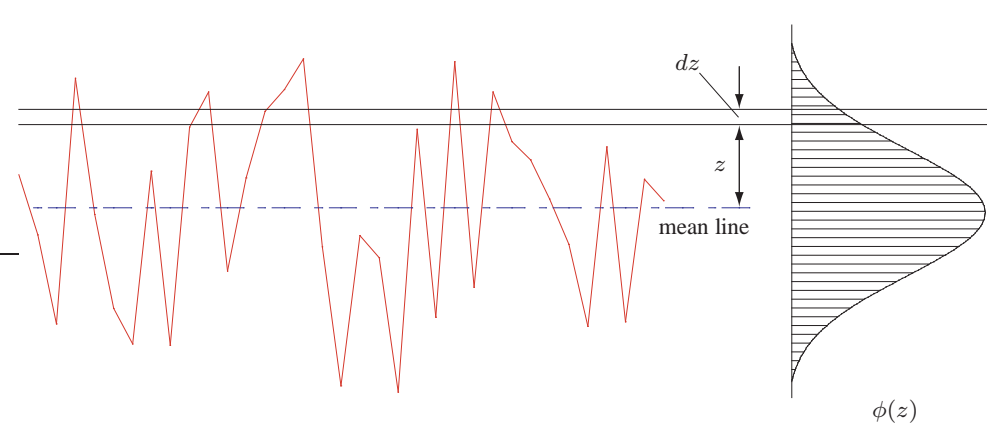

Figure 3.2: Profile heights and probability density of summits

1). Root mean square roughness $\sigma$ : This parameter, also called RMS, is the standard deviation of the height distribution of a surface from its mean line

$$
\sigma=\sqrt{\frac{1}{L} \int_{0}^{L} z^{2}(x) d x}
$$

2). Probability density function $\phi$ : The probability density function, also known as the amplitude density in statistics, represents the distribution spectrum of a profile height and can be expressed by plotting the density of the profile height shown in Figure 3.2.

In order to obtain the probability density, the height of a profile is divided into layers with small increment $d z$. Comparing the length of the profile laying between $z$ and $z+d z$ with the total length of the profile gives rise to the probability $P(z<Z<z+d z)$, thus the probability density can be written as

$$
\phi(z)=\lim _{d z \rightarrow 0} \frac{P(z<Z<z+d z)}{d z}
$$

In the GW model, it is assumed that the height distribution obeys the following Gaussian or normal probability density function

$$
\mathcal{N}\left(0, \sigma^{2}\right): \quad \phi(z)=\frac{1}{\sqrt{2 \pi \sigma^{2}}} \exp \left(-\frac{z^{2}}{2 \sigma^{2}}\right)
$$




\subsubsection{Contact of two nominally flat rough surfaces}

Consider the contact problem of two nominally flat rough surfaces which are assumed to have RMS roughness values $\sigma_{1}$ and $\sigma_{2}$ respectively, and have a separation distance $d$ between their nominal flat surfaces. The problem can be further reduced to the contact of a rigid smooth flat surface with a deformable rough flat surface of an equivalent RMS roughness (due to the Gaussian distributions)

$$
\sigma^{2}=\sigma_{1}^{2}+\sigma_{2}^{2}
$$

The height profile of the rough surface is described by the summit height $z$, the mean summit line and the probability function $\phi(z)$ as shown in Figure 3.2.

As mentioned above, all the summits are assumed to have the same radius $\beta$ and there are $N$ summits in the nominal surface area. Since the overlap between the flat surface and an asperity with height $z_{s}$ greater than the separation $d$ is $z_{s}-d$, the contact force $f$ of the summit of the asperity for a linear elastic contact is defined by the Hertzian theory as

$$
f\left(z_{s}\right)=\frac{4}{3} E \beta^{1 / 2}\left(z_{s}-d\right)^{3 / 2}
$$

where $E$ is the equivalent Young's modulus of the two contacting surfaces.

The probability of having a contact at any given asperity of height $z_{s}$ is

$$
\operatorname{prob}\left(z_{s}>d\right)=\int_{d}^{\infty} \phi\left(z_{s}\right) d z_{s}
$$

Then the total contact force experienced by the nominal surface area, in terms of separation $d$, is

$$
P(d)=N \int_{d}^{\infty} f\left(z_{s}\right) \phi\left(z_{s}\right) d z_{s}=\frac{4}{3} E N \beta^{1 / 2} \int_{d}^{\infty}\left(z_{s}-d\right)^{3 / 2} \phi\left(z_{s}\right) d z_{s}
$$

\subsubsection{Contact of two rough spheres}

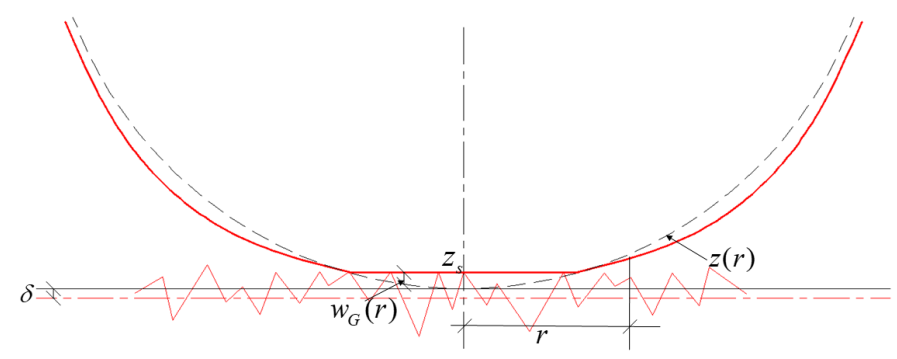

Figure 3.3: Profile of the contact between a smooth sphere and a rough surface: $\delta \leq 0$

The contact between two rough spheres can be mathematically transformed into the contact 
between a deformable smooth sphere and a nominal rigid flat rough surface as shown in Figure 3.3. The equivalent radius $R$ and the equivalent standard deviation of the asperity height distribution $\sigma$ can be obtained from the radii and roughness parameters of the two spheres by the Equation (3.4) and the follow equation

$$
\frac{1}{R}=\frac{1}{R_{1}}+\frac{1}{R_{2}}
$$

in which subscripts 1 and 2 indicate the sphere number.

Referring to Figure 3.3, $\delta$ is the separation or overlap between the non-deformed configuration of the sphere and the mean line of the flat surface. To make it compatible with the convention of the DEM, $\delta$ is assumed to be negative when the two surfaces are in separation, and positive in overlap.

The profile of the undeformed sphere (black dashed line) can be described by

$$
z(r)=\delta_{0}-\frac{r^{2}}{2 R}
$$

where $r$ is the distance from the centre to the contact point. Then the separation between the deformed sphere and the nominal flat surface at $r$ is

$$
d(r)=w_{G}(r)-\delta+\frac{r^{2}}{2 R}
$$

where $w_{G}(r)$ is the bulk deformation of the sphere. The overlap of the asperity of height $z_{s}$ at $r$ with the deformed sphere is

$$
o(r)=z_{s}-d(r)
$$

When $o(r)>0$, the contact force between the sphere and the asperity can be computed by the Hertzian theory

$$
f\left(z_{s}\right)=\frac{4}{3} E \beta^{1 / 2}\left[z_{s}-d(r)\right]^{3 / 2}
$$

in which $\beta$ is the radius of the top of the asperity and is assumed to be the same for all the asperities; and $E$ is the equivalent Young's modulus of the original two spheres. Further assume that the distribution of the asperity heights obeys the following Gaussian probability density function

$$
\phi\left(z_{s}\right)=\frac{1}{\sqrt{2 \pi \sigma^{2}}} \exp \left(-\frac{z_{s}^{2}}{2 \sigma^{2}}\right)
$$

The probability of having a contact at any given asperity of height $z_{s}$ is thus

$$
\operatorname{prob}\left(z_{s}>d(r)\right)=\int_{d(r)}^{+\infty} \phi\left(z_{s}\right) d z_{s}
$$

Then the contact pressure distribution between the sphere and the asperities over the entire 
contact area can be expressed as

$$
p_{G}(r)=C \int_{d(r)}^{+\infty}\left[z_{s}-d(r)\right]^{3 / 2} \phi\left(z_{s}\right) d z_{s}
$$

with the constant

$$
C=\frac{4}{3} E N \beta^{1 / 2}
$$

in which $N$ is the number of summits in the nominal area. The corresponding deformation $w_{G}(r)$ can be obtained from the solution to the axi-symmetric deformation of an elastic half-space as follows [169]

$$
w_{G}(r)=\frac{4}{\pi E} \int_{0}^{\bar{a}} \frac{t}{r+t} p_{G}(t) \mathbf{K}(k) d t
$$

where $\mathbf{K}(k)$ is the complete elliptic integral of first kind with elliptic modulus

$$
k=\frac{2 \sqrt{r t}}{r+t}
$$

and $\bar{a}$ is the radius of the contact area. By integrating the pressure distribution over the contact area, the total contact force $P_{G}$ between the sphere and the rough surface with overlap $\delta$ can be obtained by

$$
P_{G}(\delta, \sigma)=\int_{0}^{\bar{a}} 2 \pi r p_{G}(r) d r
$$

\subsubsection{A simple extension to positive overlap and theoretical inconsistency}

Although the classic GW model has been validated (mainly qualitatively though), extended and applied to many applications, see for instance [165, 169-171], it is not clear whether the classic GW model is still valid or not for $\delta>0$, i.e. when there is a positive overlap between the sphere and the nominal flat surface. In this subsection, the simple extension of the GW model without any modification is considered and a theoretical inconsistency will be identified which leads to the proposal of the extended elastic GW model in the next chapter.

For the convenience of later reference, the corresponding Hertzian solutions for the smooth spheres with $\delta>0$ are given below:

Contact radius:

$$
a_{H}=\sqrt{R \delta}
$$

Pressure distribution $p_{H}(r)$ :

$$
p_{H}(r)= \begin{cases}p_{H_{0}}\left(1-\frac{r^{2}}{a_{H}^{2}}\right)^{1 / 2} ; \quad p_{H_{0}}=\frac{2 E}{\pi} \frac{a_{H}}{R} ; & r \leq a_{H} \\ 0 ; & r>a_{H}\end{cases}
$$


Deformation distribution:

$$
w_{H}(r)= \begin{cases}w_{H_{0}}\left(1-\frac{r^{2}}{2 a_{H}^{2}}\right)=\delta-\frac{r^{2}}{2 R} ; \quad w_{H_{0}}=\delta ; & r \leq a_{H} \\ \frac{a_{H}^{2}}{\pi R}\left[\frac{r^{2}}{a_{H}^{2}}-1+\left(2-\frac{r^{2}}{a_{H}^{2}}\right) \sin ^{-1}\left(\frac{a_{H}}{r}\right)\right] ; & r>a_{H}\end{cases}
$$

Total force:

$$
P_{H}(\delta)=\frac{4}{3} E a_{H} \delta=\frac{4}{3} E \sqrt{R} \delta^{3 / 2}
$$

Under the simple extension of the GW model, it is theoretically important that the GW model can reduce to the Hertzian solution for smooth spheres when the roughness $\sigma=0$. However, this is not apparent. In fact, it is easy to verify that directly setting $w_{G}(r)$ to be the Hertzian deformation $w_{H}(r)$ in (3.10) and (3.15) gives rise to a zero pressure distribution $p_{G}(r)=0$, which is obviously incorrect when $\delta>0$. The classic GW model does recover the Hertzian solution, but as the limit when $\sigma \rightarrow 0$ if the parameter $\mu$ is assumed to be fixed, $\mu$ is a non-dimensional roughness parameter refer to Equation (3.38).

Assuming that when $\sigma$ is close to zero, both the pressure and deformation distributions of the sphere are close to the Hertzian solutions for the smooth contact case, and can be expressed as

$$
p_{G}(r)=p_{H}(r)-\Delta p(r) ; \quad \text { with } \Delta p(r) \ll p_{H}(r)
$$

and

$$
w_{G}(r)=w_{H}(r)-\Delta w(r) ; \quad \text { with } \Delta w(r) \ll w_{H}(r)
$$

Note that the minus signs on both the right hand sides of the above two expressions are deliberately assumed and the implication will be highlighted at the end of this subsection.

Note that when $\sigma \rightarrow 0$, the zero-centred normal probability distribution function $\phi\left(z_{s}\right)$ tends to the Dirac delta function, denoted here as $\Delta\left(z_{s}\right)$, so that (3.15) becomes

$$
p_{G}(r)=C \int_{d(r)}^{\infty}\left[z_{s}-d(r)\right]^{3 / 2} \Delta\left(z_{s}\right) d z_{s}=C\left[\delta-w_{G}(r)-\frac{r^{2}}{2 R}\right]^{3 / 2}
$$

From (3.10) and (3.11) it follows

$$
p_{H}(r)-\Delta p(r)=C[\Delta w(r)]^{3 / 2}
$$

thus

$$
p_{H}(r) \approx C[\Delta w(r)]^{3 / 2}
$$

or

$$
\Delta w(r) \approx\left[\frac{p_{H}(r)}{C}\right]^{2 / 3}=\left(\frac{p_{0}}{C}\right)^{2 / 3}\left(1-\frac{r^{2}}{a^{2}}\right)^{1 / 3}
$$


and the maximum difference between $\Delta w(r)$ and $w_{H}(r)$ appears at $r=0$ with

$$
\Delta w(0)=\left(\frac{16 \sqrt{2}}{3 \pi \mu} \frac{\sigma}{\delta}\right)^{2 / 3} w_{0}
$$

For a given overlap $\delta>0$ and a fixed $\mu$, it follows that

$$
\lim _{\sigma \rightarrow 0} \Delta w(r)=0
$$

The linear relationship between $w(r)$ and $p(r)$ in (3.17) is also applicable to $\Delta w(r)$ and $\Delta p(r)$ :

$$
\Delta w(r)=\frac{4}{\pi E} \int_{0}^{\bar{a}} \frac{t}{r+t} \Delta p(r) \mathbf{K}(k) d t
$$

Although an explicit expression for $\Delta p(r)$ may not be available, the numerical simulation shown below indicates that $\Delta p(r)$ is almost constant except in a very small region at $r=a$. So it may assume that

$$
\Delta p(r) \approx \Delta p(0)
$$

While from (3.32), it has

$$
\Delta p(0)=\frac{E}{2 a} \Delta w(0)
$$

so

$$
\Delta p(r) \approx \frac{E}{2 a} \Delta w(0)
$$

Thus it is concluded that the classic GW model converges to the Hertzian solution when $\sigma \rightarrow 0$ :

$$
\lim _{\sigma \rightarrow 0} w_{G}(r)=w_{H}(r) ; \quad \lim _{\sigma \rightarrow 0} p_{G}(r)=p_{H}(r)
$$

Nevertheless, as $p_{G}$ and $w_{G}$ are approaching to $p_{H}$ and $w_{H}$ but from the negative side (refer to (3.24) and (3.25), the following conclusion holds when $\delta$ is small

$$
P_{G}(\delta)<P_{H}(\delta)
$$

Clearly this is not physical since the contact force for the two rough spheres cannot be smaller than the smooth case for the same nominal overlap $\delta$, thus revealing that the classic GW model is not theoretically valid for $\delta>0$, at least for small $\sigma$.

\subsection{Non-dimensional forms}

There are three parameters in Equation (3.15) that are associated with the surface roughness: $N, \beta$ and $\sigma$, but only $\sigma$ and $N \beta^{1 / 2}$ are independent. $\sigma$ has a clear geometric meaning and can be reasonably defined for a given pair of surfaces (spheres). However this may not be the case for $N \beta^{1 / 2}$, and in particular, its value range can be very large. 
A non-dimensional parameter, $\mu$, is defined in [165] as

$$
\mu=\frac{8}{3} \sigma N \sqrt{2 R \beta}
$$

Compared to $N \beta^{1 / 2}, \mu$ has a much narrower range, as stated in [169], therefore is adopted here to replace $N \beta^{1 / 2}$ as the second independent parameter. Since

$$
N \beta^{1 / 2}=\frac{3 \mu}{8 \sqrt{2 R} \sigma}
$$

the coefficient $C$ defined by (3.16) can be expressed in terms of $\sigma$ and $\mu$ as

$$
C=\frac{\mu}{\sqrt{8 R} \sigma} E
$$

Thus in this work, $\delta, \sigma$ and $\mu$ are chosen to be the three input parameters.

It may often provide better physical insights and is more computationally efficient if a formula is made dimensionless. To achieve this for the GW formulations, two non-dimensionalised forms will be described below. The first one, termed the $\sigma$-form, is mainly based on the original version proposed by Greenwood and Tripp [165] where $\sigma$ is involved as the main scaling factor. The second one, termed the $\delta$-form, is similar but uses $\delta$ as the main scaling factor.

These two forms are closely associated with the non-dimensional parameter $\alpha$ introduced in [169] and its reciprocal $\alpha^{\prime}$ defined as

$$
\alpha=\frac{\sigma}{\delta} ; \quad \alpha^{\prime}=\frac{1}{\alpha}=\frac{\delta}{\sigma}
$$

These two parameters will appear in the two non-dimensional forms respectively. For an

Table 3.1: The scaling factors in two non-dimensional forms

\begin{tabular}{c|c|c}
\hline$q^{*}$ & $\sigma$-form & $\delta$-form \\
\hline$\delta^{*}$ & $\sigma$ & - \\
$\sigma^{*}$ & - & $\delta$ \\
$w^{*}$ & $\sigma$ & $\delta$ \\
$z_{s}^{*}$ & $\sigma$ & $\delta$ \\
$\phi^{*}\left(z_{s}^{*}\right)$ & $1 / \sigma$ & $1 / \delta$ \\
$r^{*}$ & $\sqrt{2 R \sigma}$ & $\sqrt{2 R \delta}$ \\
$\bar{a}^{*}$ & $\sqrt{2 R \sigma}$ & $\sqrt{2 R \delta}$ \\
$p^{*}$ & $E \sqrt{\sigma / 8 R}$ & $E \sqrt{\delta / 8 R}$ \\
$P^{*}$ & $P_{H}(\sigma)$ & $P_{H}(\delta)$ \\
\hline & \multicolumn{2}{|c}{}
\end{tabular}

arbitrary physical quantity or function $q$, its non-dimensionalised version $q^{*}$ can be determined by a scaling factor $\lambda_{q}$ :

$$
q=\lambda_{q} q^{*}
$$


Table 3.1 lists the scaling factors for those quantities in the two non-dimensional forms. The non-dimensional expressions of the functions $p_{G}(r), w_{G}(r)$ and $P_{G}$ for the two forms in terms of the non-dimensional parameters $\alpha$ (or $\alpha^{\prime}$ ) and $\mu$ are presented below.

$$
p_{G}^{*}\left(r^{*}, \alpha^{\prime}\right)=\mu \int_{w^{*}\left(r^{*}, \alpha^{\prime}\right)+r^{* 2}}^{\infty}\left[z_{s}^{*}-w^{*}\left(r^{*}, \alpha^{\prime}\right)-r^{* 2}\right]^{3 / 2} \phi\left(z_{s}^{*}-\alpha^{\prime}\right) d z_{s}^{*}
$$

The $\sigma$-form: $\quad w_{G}^{*}\left(r^{*}, \alpha^{\prime}\right)=\frac{2}{\pi} \int_{0}^{\bar{a}^{*}} \frac{t^{*}}{t^{*}+r^{*}} p^{*}\left(t^{*}, \alpha^{\prime}\right) \mathbf{K}(k) d t^{*}$

$$
P_{G}^{*}\left(\alpha^{\prime}, \mu\right)=\frac{3 \sqrt{2}}{8} \int_{0}^{\bar{a}^{*}} 2 \pi r^{*} p^{*}\left(r^{*}, \alpha^{\prime}\right) d r^{*}
$$

$$
p_{G}^{*}\left(r^{*}, \alpha\right)=\frac{\mu}{\alpha} \int_{w^{*}\left(r^{*}, \alpha\right)+r^{* 2}}^{\infty}\left[z_{s}^{*}-w^{*}\left(r^{*}, \alpha\right)-r^{* 2}\right]^{3 / 2} \phi\left(z_{s}^{*}-1\right) d z_{s}^{*}
$$

The $\delta$-form: $\quad w_{G}^{*}\left(r^{*}, \alpha\right)=\frac{2}{\pi} \int_{0}^{\bar{a}^{*}} \frac{t^{*}}{t^{*}+r^{*}} p^{*}\left(t^{*}, \alpha\right) \mathbf{K}(k) d t^{*}$

$$
P_{G}^{*}(\alpha, \mu)=\frac{3 \sqrt{2}}{8} \int_{0}^{\bar{a}^{*}} 2 \pi r^{*} p^{*}\left(r^{*}, \alpha\right) d r^{*}
$$

Note that $w_{G}^{*}$ and $P_{G}^{*}$ have the same expression, whilst only $p_{G}^{*}$ is slightly different in the two forms. Most importantly, the total contact force between two rough spheres can now be written as

$$
P_{G}(\delta, \sigma, \mu)=P_{H}(\delta) P_{G}^{*}(\alpha, \mu)=P_{H}(\sigma) P_{G}^{*}\left(\alpha^{\prime}, \mu\right)
$$

i.e. the Hertzian load $P_{H}$ for the smooth contact with the same overlap $\delta$, or with the roughness $\sigma$ as the equivalent overlap, is acting as the scaling factor for the total force $P_{G}$ in each form. Particularly, the overlap $\delta$ (or the roughness $\sigma$ ) has now been separated from the other two non-dimensional parameters $\alpha$ (or $\alpha^{\prime}$ ) and $\mu$, and therefore the total contact load $P_{G}\left(\delta, \alpha^{\prime}, \mu\right)$ (or $P_{G}\left(\sigma, \alpha^{\prime}, \mu\right)$ ) as a function of the three variables can now be obtained by simply evaluating the non-dimensional load $P_{G}^{*}(\alpha, \mu)$ (or $P_{G}^{*}\left(\alpha^{\prime}, \mu\right)$ ), only involving $\alpha$ (or $\left.\alpha^{\prime}\right)$ and $\mu$, and then multiplying it by the Hertzian load $P_{H}(\delta)$ (or $P_{H}(\sigma)$ ). The total computational costs therefore can be reduced substantially by an order of magnitude in comparison with the original formulations. In addition, the non-dimensional forms can make the curve fitting of $P_{G}(\delta, \alpha, \mu)\left(\right.$ or $\left.P_{G}\left(\sigma, \alpha^{\prime}, \mu\right)\right)$ more accurate in the next stage.

\subsection{Numerical Solutions and Computational Issues}

Due to the inter-dependence between the pressure $p_{G}(r)$ and the deformation $w_{G}(r)$, and the non-integrable part involving the Gaussian distribution, as shown in Equations (3.15) and (3.17), an explicit expression between the overlap $\delta$ and the contact force $P_{G}$ cannot be established. Thus, numerical solutions must be sought to obtain the force $P_{G}$ for a given set of values for $\delta, \sigma$ (or $\alpha$ ) and $\mu$. In what follows, the original formulations of the GW model are used for discussion, but the proposed procedure and relevant numerical techniques are 
equally applicable to the two non-dimensional forms introduced in Section 3.3.

\subsubsection{Numerical solutions of the pressure and deformation distributions and the contact force}

Because Equations (3.15) and (3.17) are coupled to implicitly define the pressure distribution $p_{G}(r)$ in terms of the deformation of the sphere $w_{G}(r)$ over the contact area, both equations need to be solved simultaneously to obtain a numerical solution. Although this can be fulfilled in a different manner, the Newton-Raphson method will be employed in this work due to its quadratic convergence.

Note that the contact radius $\bar{a}$ may not be known in prior because the contact radius for rough surface contact may not be determined precisely. Although a sufficiently large value of $\bar{a}$ can be estimated based on the given overlap $\delta$ and the roughness $\sigma$, an appropriate value should be used to achieve a high numerical accuracy when the contact region is discretised as described below.

Firstly, the interval of the contact area $[0, \bar{a}]$ is discretised into $m$ discrete points $\mathbf{r}_{m}=$ $\left[r_{1}, \cdots, r_{m}\right]^{T}$. In this work these points are taken to be the integration points (or abscissae) of the chosen numerical integration quadrature for the integral in Equation (3.17) which will be discussed later, and the corresponding weights are assumed to be $\mathbf{s}_{m}=\left[s_{1}, \cdots, s_{m}\right]^{T}$. Then Equation (3.15) can be discretized as

$$
p_{G i}=C \int_{d_{i}}^{\infty}\left(z_{s}-d_{i}\right)^{3 / 2} \phi\left(z_{s}\right) d z_{s} \equiv C g\left(w_{G i}\right)
$$

where

$$
g\left(w_{G i}\right)=\int_{d_{i}}^{\infty}\left(z_{s}-d_{i}\right)^{3 / 2} \phi\left(z_{s}\right) d z_{s} ; d_{i}=w_{G i}-\delta+r_{i}^{2} /(2 R)
$$

and Equation (3.17) becomes

$$
w_{G i}=\frac{4}{\pi E} \sum_{j=1}^{m} s_{j} \alpha_{i j} p_{G j}
$$

where the coefficients $\alpha_{i j}$ are

$$
\alpha_{i j}=\frac{r_{j}}{r_{i}+r_{j}} \mathbf{K}\left(k_{i j}\right) ; \quad k_{i j}=\frac{2 \sqrt{r_{i} r_{j}}}{r_{i}+r_{j}}
$$

Thus the equation to be satisfied at discrete point $i$ is governed by

$$
F_{i}\left(p_{G 1}, \cdots, p_{G m}\right)=p_{G_{i}}-C g\left(w_{G_{i}}\right)=0
$$

Since this equation has to be satisfied at all the discrete points, $r_{i}(i=1, \cdots, m)$, it leads to a nonlinear system of equations in vector format

$$
\mathbf{F}\left(\mathbf{p}_{G}\right)=\mathbf{p}_{G}-C \mathbf{g}\left(\mathbf{w}_{G}\right)=0
$$


where the four vectors involved are

$\mathbf{F}=\left[F_{G_{q}}, \cdots, F_{G_{m}}\right]^{T} ; \mathbf{p}=\left[p_{G_{1}}, \cdots, p_{G_{m}}\right]^{T} ; \mathbf{w}=\left[w_{G 1}, \cdots, w_{G m}\right]^{T} ; \mathbf{g}(\mathbf{w})=\left[g\left(w_{G 1}\right), \cdots, g\left(w_{G m}\right)\right]^{T}$

To solve the above nonlinear system of equations in terms of $\mathbf{p}_{G}$ by the Newton-Raphson method, the function $\mathbf{F}$ is expanded by the Taylor series in the neighbourhood of $\mathbf{p}_{G}$ with an infinitesimal increment $\delta \mathbf{p}$

$$
\mathbf{F}\left(\mathbf{p}_{G}+\delta \mathbf{p}\right)=\mathbf{F}\left(\mathbf{p}_{G}\right)+\mathbf{J} \delta \mathbf{p}+\mathrm{O}\left(\delta \mathbf{p}^{2}\right)
$$

where $\mathbf{J}$ is the Jacobian matrix of the vector function $\mathbf{F}$

$$
\mathbf{J}=\nabla \mathbf{F} ; \text { or } J_{i j}=\frac{\partial F_{i}}{\partial p_{G j}}
$$

By ignoring the 2nd and higher order terms in (3.55), the increment $\delta \mathbf{p}$ can be obtained by

$$
\delta \mathbf{p}=-\mathbf{J}^{-1} \mathbf{F}\left(\mathbf{p}_{G}\right)
$$

The final solution $\mathbf{p}_{G}$ is achieved when the iterative process converges starting from a trial solution that can be chosen to be the Hertzian pressure distribution. Then the total contact force can be obtained by numerically integrating the converged discrete pressure distribution $\mathbf{p}_{G}$ over the entire contact area

$$
P_{G}(\delta)=2 \pi \sum_{j=1}^{m} s_{j} r_{j} p_{G_{j}}
$$

\subsubsection{Computational issues}

There are several computational issues involved in the above numerical procedure that may have some significant impact on the overall computational efficiency and accuracy so thus need to be discussed in detail.

\section{Numerical integrations}

Three integrals involved in Equations (3.15) (3.17) need to be evaluated numerically. Although many numerical integration quadratures can be used, such as the trapezium or Simpson rule with equally spaced integration points, the Gaussian quadrature is adopted in the current work due to its high algebraic accuracy. As the two integrals in Equations (3.15) and (3.17) have the same integral domain which is the contact area $[0, \bar{a}]$, the same Gaussian points and weights are used. Note that this is also the requirement of the Newton-Raphson solution procedure outlined in the previous subsection.

The integral in (3.19) has a different integral domain and thus should be evaluated using a different number of Gaussian points. Although the upper bound of the domain should be 
infinity in theory, a limited value based on the given roughness $\sigma$ can be adopted instead.

\section{Evaluation of the Jacobian matrix}

The Jacobian matrix $\mathbf{J}$ needs to be evaluated at each Newton-Raphson iteration. However, it is difficult to obtain the analytical expression. In this work, a finite difference approximation to $\mathbf{J}$ is employed. Let $\mathbf{J}_{j}$ be the $j$-th column of $\mathbf{J}$, and $\mathbf{e}_{j}=[0, \cdots, 1, \cdots, 0]^{T}$ be a unit vector with only the $j$-component being unity. Then $\mathbf{J}_{j}$ is approximated by

$$
\mathbf{J}_{j}=\frac{1}{\epsilon_{j}}\left[\mathbf{F}\left(\mathbf{p}+\epsilon_{j} \mathbf{e}_{j}\right)-\mathbf{F}(\mathbf{p})\right], \quad j=1, \ldots, m
$$

where

$$
\epsilon_{j}=\epsilon \max \left\{p_{j}, \epsilon\right\}
$$

in which $\epsilon$ is a perturbation parameter. Note that $\epsilon$ should be neither too large to sacrifice the quadratic convergence of the Newton-Raphson method, nor too small to cause numerical instability. The numerical test, to be presented in the next section, shows that $\epsilon=10^{-6}$ is sufficient to maintain the quadratic convergence without causing any numerical instability.

\section{Determination of coefficients $\alpha_{i j}$}

The coefficients $\alpha_{i j}$ defined in (3.52) play a crucial role in the current numerical solution procedure. An efficient approach to determine their values are described below. Introducing a ratio $\lambda_{i j}=r_{i} / r_{j}, \alpha_{i j}$ can now be expressed in a slightly different form

$$
\alpha_{i j}=\frac{1}{1+\lambda_{i j}} \mathbf{K}\left(k_{i j}\right) ; \quad k_{i j}=\frac{2 \sqrt{\lambda_{i j}}}{1+\lambda_{i j}}
$$

As $\lambda_{i j}$ is non-dimensional and fixed for a given $m$ of the integration quadrature regardless of the contact radius $\bar{a}, \alpha_{i j}$ are also non-dimensional and fixed. Note that $k_{i j}=k_{j i}$, thus $\mathbf{K}\left(k_{i j}\right)=\mathbf{K}\left(k_{j i}\right)$. Since it is computationally intensive to obtain the value of the elliptic function $\mathbf{K}(k)$, utilising the symmetry of $k_{i j}$ can halve the computational costs in evaluating $\alpha_{i j}$. Note that a relatively effective way to compute $\mathbf{K}(k)$ is by the arithmetic-geometric mean described in [172].

However, a singularity problem occurs when evaluating the diagonal terms $\alpha_{i i}$ since $\lambda_{i i}=$ $k_{i i}=1$, while $\mathbf{K}(1)$ is infinity. This singularity is avoided in [165] by introducing an auxiliary function $\mathcal{L}(\xi)$, but its inverse has to be found numerically in order to evaluate $w(r)$. In [173] or [174], the problem is avoided by assuming that the pressure is constant over each discretised element or element, and therefore the integral (3.17) over the element can be explicitly expressed, but at the expense of a reduced solution accuracy. In this work, the singularity problem is resolved based on the fact that the Hertzian pressure and deformation distributions are the analytical solutions to (3.17): for an arbitrarily given Hertzian pressure 


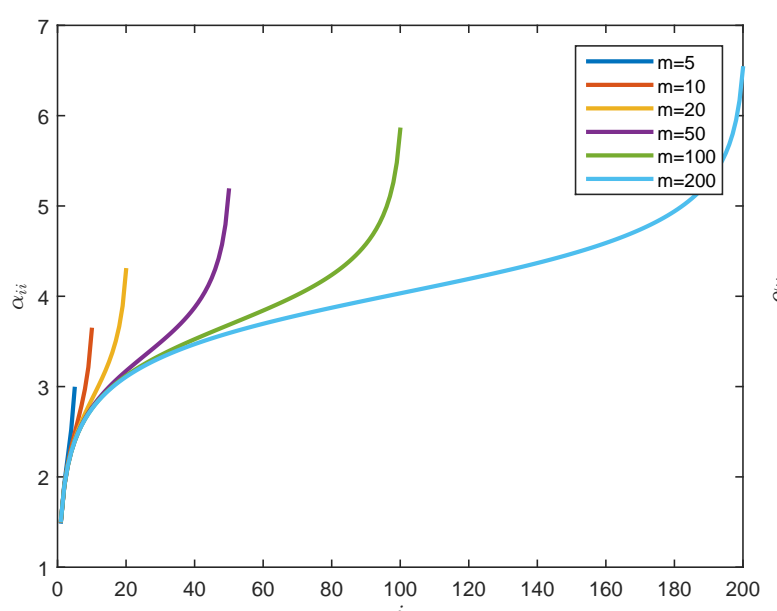

(a)

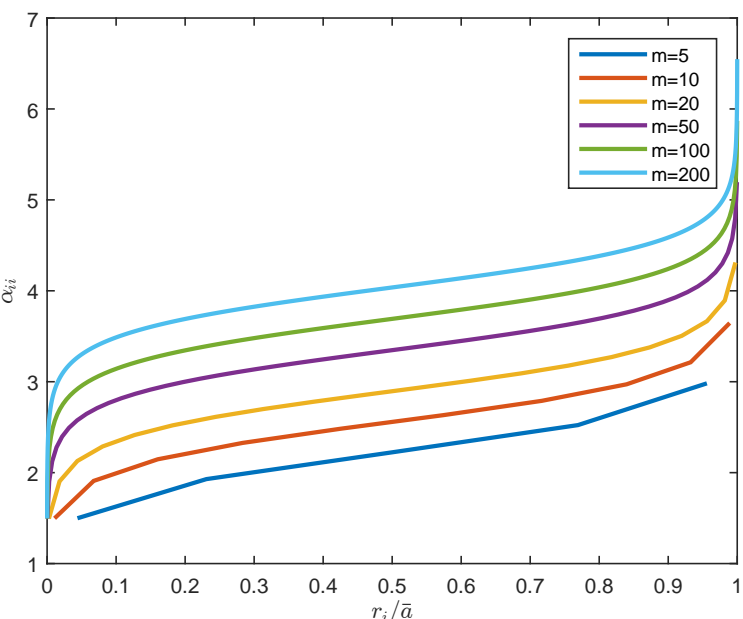

(b)

Figure 3.4: Distribution of diagonal coefficients $\alpha_{i i}$

distribution

$$
\bar{p}_{H}(r)=\bar{p}_{0}\left(1-r^{2} / \bar{a}^{2}\right)^{1 / 2}
$$

over the contact region $[0, \bar{a}]$, the deformation from $(3.17)$ is given by the Hertzian solution

$$
\bar{w}_{H}(r)=\bar{w}_{0}\left(1-r^{2} / 2 \bar{a}^{2}\right)
$$

where $\bar{w}_{0}=\pi \bar{p}_{0} \bar{a} / 2 E$. Thus it is natural to require that $\alpha_{i i}$ be determined in such a manner so that for the given Hertzian pressure distribution $\bar{p}_{H}(r)$, the calculated $w_{G i}$ from (3.51) should be equal to $\bar{w}_{H}\left(r_{i}\right)$ :

$$
\bar{w}_{H}\left(r_{i}\right)=\frac{4}{\pi E} \sum_{j=1}^{m} s_{j} \alpha_{i j} \bar{p}_{H}\left(r_{j}\right)
$$

which leads to

$$
\alpha_{i i}=\frac{1}{s_{i} \bar{p}_{H}\left(r_{i}\right)}\left[\frac{\pi E}{4} \bar{w}_{H}\left(r_{i}\right)-\sum_{j=1, j \neq i}^{m} s_{j} \alpha_{i j} \bar{p}_{H}\left(r_{j}\right)\right]
$$

It can be verified that $\alpha_{i i}(i=1, \ldots, m)$ are independent of the contact radius $\bar{a}$ and the material property $E$ as expected. The distribution of $\alpha_{i i}$ in terms of $i$ or the (scaled) position $x_{i} / \bar{a}$ in the contact region $[0, \bar{a}]$ against the number of integration points $m$ in the Gaussian quadrature is illustrated in Figure 3.4, indicating that the lower terms converge when $m$ increases.

In summary, all the coefficients $\alpha_{i j}$ are solely determined by the number of integration points $m$ (and their positions) for any chosen integration quadrature and thus can be pre-calculated when $m$ is given and used for any overlap and surface roughness. This feature, together with the property $\mathbf{K}\left(k_{i j}\right)=\mathbf{K}\left(k_{j i}\right)$, significantly increases the computational efficiency of the preceding numerical solution procedure. The specific approach to determining the diagonal terms $\alpha_{i i}$ not only eliminates the singularity problem, but also maintains a high numerical accuracy of the integration quadrature. 
Table 3.2: The pressure errors in the Newton-Raphson iterations for different $\alpha(\mu=4)$

\begin{tabular}{c|c|c|c}
\hline Iteration & $\alpha=0.01$ & $\alpha=0.1$ & $\alpha=1.0$ \\
\hline 1 & $7.26 \mathrm{e}-2$ & $1.77 \mathrm{e}-1$ & $2.36 \mathrm{e}-1$ \\
2 & $3.81 \mathrm{e}-2$ & $5.00 \mathrm{e}-2$ & $1.27 \mathrm{e}-2$ \\
3 & $7.53 \mathrm{e}-3$ & $4.38 \mathrm{e}-3$ & $8.28 \mathrm{e}-5$ \\
4 & $4.68 \mathrm{e}-4$ & $2.81 \mathrm{e}-5$ & $2.11 \mathrm{e}-11$ \\
5 & $1.80 \mathrm{e}-6$ & $8.58 \mathrm{e}-10$ & $1.14 \mathrm{e}-17$ \\
6 & $2.42 \mathrm{e}-11$ & $2.77 \mathrm{e}-16$ & \\
7 & $1.40 \mathrm{e}-15$ & & \\
\hline
\end{tabular}

\subsection{Numerical Results and Validation}

This section presents and validates some numerical results obtained following the numerical procedures proposed in the previous sections to ensure that the computed contact forces $P_{G}(\delta, \sigma, \mu)$ are sufficiently accurate to be curve-fitted in the next section. The validation of some results will be conducted against the known results presented in the literature. There are several parameters involved in the numerical procedure proposed in the preceding section that need to be selected appropriately to ensure a high solution accuracy and overall computational efficiency. Selections of their values will also be discussed.

\subsubsection{Convergence of the Newton-Raphson procedure}

The Newton-Raphson method should exhibit a quadratic convergence when properly implemented. This property may be affected if the chosen value of the perturbation parameter $\epsilon$ in (3.59) is too large. The residual error at iteration $i$ is defined as

$$
\epsilon_{i}=\left\|\delta \mathbf{p}_{G_{i}}\right\| /\left\|\mathbf{p}_{G_{i}}\right\|
$$

where $\delta \mathbf{p}_{i}$ is the pressure increment at the iteration and $\mathbf{p}_{G i}$ is the updated pressure after the iteration. The Hertzian pressure distribution $p_{H}(r)$ is taken as the initial guess for $p_{G}(r)$. The perturbation parameter $\epsilon$ is set to be $10^{-6}$. For a fixed $\mu=4$ and $m=100$, the history of the residual errors during the iterations for different $\alpha$ is recorded in Table 3.2. It is clear that a quadratic convergence is achieved for all the $\alpha$ values, indicating that $\epsilon=10^{-6}$ is an appropriate value. It is also evident that faster convergent speeds are achieved for larger $\alpha$, and the typical number of iterations needed to reach an accuracy of around $10^{-6}$ is about 4 or 5 .

\subsubsection{Selections of numerical parameters}

The following parameters need to be specified in the numerical procedure: 1) the contact radius $\bar{a} ; 2$ ) the number of Gaussian integration points $m$ used in the evaluation of the integrals 
Table 3.3: The computed $P_{G}^{*}(\alpha, \mu)$ for different numbers of Gaussian points $m(\mu=4)$

\begin{tabular}{c|c|c|c}
\hline$m$ & $\alpha=0.1$ & $\alpha=1$ & $\alpha=5$ \\
\hline 5 & $\mathbf{0 . 9 7 0 1 2 5 5}$ & $\mathbf{2 . 0 1 4 3 6 3 5}$ & $\mathbf{1 1 . 0 7 7 9 9 6}$ \\
10 & $\mathbf{0 . 9 7 7 0 7 9 1}$ & $\mathbf{2 . 0 1 3 9 9 6 5}$ & $\mathbf{1 1 . 0 7 0 6} 76$ \\
20 & $\mathbf{0 . 9 7 6 9 6 5 9}$ & $\mathbf{2 . 0 1 3 8 8 6 8}$ & $\mathbf{1 1 . 0 7 1 6} 60$ \\
50 & $\mathbf{0 . 9 7 6 9 5 1 6}$ & $\mathbf{2 . 0 1 3 8 7 0 9}$ & $\mathbf{1 1 . 0 7 1 7 9 8}$ \\
100 & $\mathbf{0 . 9 7 6 9 5 0 7}$ & $\mathbf{2 . 0 1 3 8 6 9 9}$ & $\mathbf{1 1 . 0 7 1 8 0 6}$ \\
200 & $\mathbf{0 . 9 7 6 9 5 0 6}$ & $\mathbf{2 . 0 1 3 8 6 9 7}$ & $\mathbf{1 1 . 0 7 1 8 0 7}$ \\
\hline
\end{tabular}

(3.17) and (3.19); and 3) the number of Gaussian integration points and the upper bound of the integral domain for the integral (3.15).

The pressure distribution $p_{G}(r)$ reduces when $r$ increases and further drops to a very small or zero value. As the contact radius $\bar{a}$ is unknown in prior, it should be specified sufficiently large so that the actual contact region is fully covered, but not too large to cause a large portion of the (nearly) zero-pressure region because the integration or discretisation points located in the region will be wasted. The follow formula to determine $\bar{a}$ appears to work well:

$$
\bar{a}=\left(1.75+\min \left\{\alpha,\left|\alpha^{\prime}\right|\right\}\right) a
$$

where $a$ is the contact radius of a smooth contact case with the same overlap $\delta$ (for $0<\alpha<1$ ) or the equivalent overlap $\sigma$ (for $\alpha^{\prime}=1 / \alpha<1$ ).

The number of Gaussian integration points for the integral (3.15) is chosen to be 10. The upper bound is taken to be $5 \sigma$, as this will statistically cover $99.99994 \%$ of possible peak heights, and thus should not affect the final solution accuracy.

The number of Gaussian points $m$ has a major influence on the computational costs and the accuracy of the computed total force $P_{G}$, or $P_{G}^{*}(\alpha, \mu)$. In order to select a proper value, the following accuracy convergence test has been conducted: different numbers of $m$ are used to compute $P^{*}(\alpha, \mu)$ for a number of combinations of $\alpha$ and $\mu$ values. The results are presented in Table 3.3, in which the significant digits of the computed force for each $m$ are highlighted which is obtained by comparing the force value with the one for the next $m$. It appears that $m=20$ is required to obtain an accuracy of about $10^{-5}$, but it is remarkable that even $m=5$ can achieve an accuracy of $10^{-3}$. Nevertheless, to ensure that all the results are sufficiently accurate, $m$ is taken to be 200 in all the subsequent computations.

\subsubsection{Comparisons of pressure distributions and effective contact radii}

Some selected numerical results obtained in this work are validated by comparing them against those presented by Johnson in [169], including: 1) the maximum contact pressure $p_{G}(0)$ normalised by the maximum Hertzian pressure $p_{0}$ for different $\alpha$ and $\mu$; and 2) the effective contact radius $a^{*}$ normalised by the Hertzian contact radius $a$ as a function of $\alpha$ and $\mu$. The 


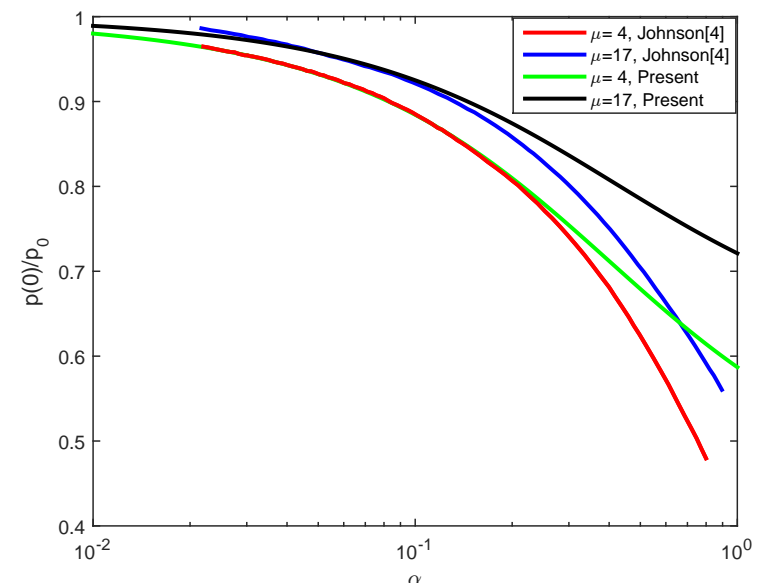

(a) $10^{-2} \leq \alpha \leq 1 ; \mu=4,17$

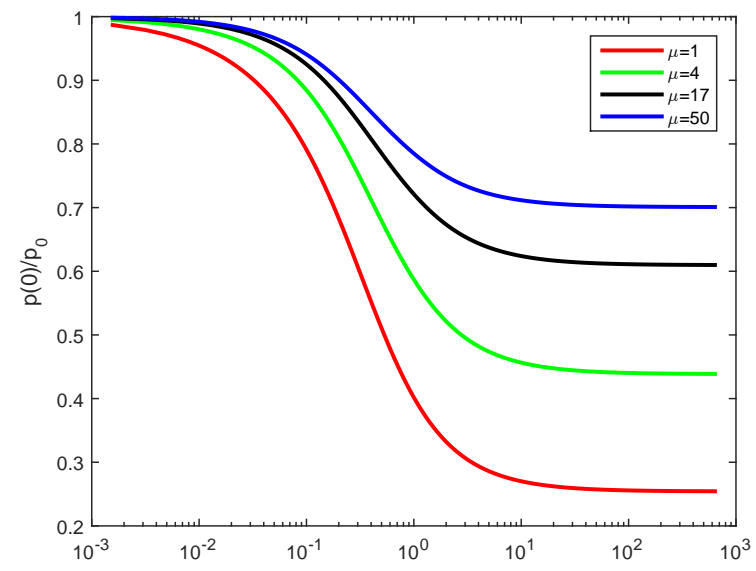

(b) $10^{-3} \leq \alpha<10^{3} ; \mu=1,4,17,50$

Figure 3.5: The ratio of the maximum contact pressure $p_{G}(0)$ to the maximum Hertzian pressure $p_{0}$ against $\alpha$ and $\mu$ : (a) comparison of the present work with Johnson's for $\alpha<1$ and two $\mu$; (b) the present work for wider ranges of $\alpha$ and $\mu$

maximum Hertzian pressure $p_{0}$ and contact radius $a$ are obtained from smooth sphere contact under the same contact load $P_{G}$. The effective contact radius $a^{*}$ is arbitrarily defined by Greenwood and Tripp [165] as

$$
a^{*}=\frac{3 \pi \int_{0}^{\bar{a}} r p_{G}(r) d r}{4 \int_{0}^{\bar{a}} p_{G}(r) d r}
$$

Figure 3.5(a) depicts a comparison of the ratio $p_{G}(0) / p_{0}$ for $10^{-3}<\alpha \leq 1$ and two values of $\mu=4$ and 17 between the present work and those presented in Figure 13.12 on page 420 in [169]. It shows an excellent agreement in the range $0.02<\alpha<0.2$ for the curve $\mu=4$, and in a narrower range $0.045<\alpha<0.065$ for the curve $\mu=17$. However, the difference between the present work and the referenced work [169] becomes noticeable and increases when $\alpha$ increases towards $\alpha=1$ for $\mu=4$ and 17, and when $\alpha$ decreases from 0.045 for $\mu=17$. No comparison, however, can be made for $\alpha>1$ as no corresponding results are available in the referenced work.

Nevertheless, in order to shed a further insight into the contact behaviour of rough surfaces, the maximum effective pressure $p_{G}(0)$ over the Hertzian pressure $p_{0}$ from the current work is provided in Figure 3.5(b) for a much wider range of $\alpha\left(10^{-3}<\alpha<10^{3}\right)$ and a larger set of $\mu(=1,4,17$ and 50$)$. The figure shows that the ratio $p_{G}(0) / p_{0}$ increases towards 1 when $\alpha$ decreases to zero regardless of $\mu$, as proved in Section 3.2.4; while $\alpha$ decreases with the increase of $\alpha$ but asymptotically reaches a non-zero limit value that monotonically increases with $\mu$. On the contrary, it may be reasonably deduced from the two curves in the referenced work that when $\alpha$ increases the ratio decreases towards zero in an accelerated manner.

Figure 3.6(a) shows a comparison of the ratio $a^{*} / a$ for $10^{-3}<\alpha \leq 1$ and two values of $\mu=4$ and 17 between the present work and those presented in Figure 13.13 on page 421 in [169]. Again, it shows a very good agreement in the range $0.02<\alpha<0.2$ for the curve $\mu=4$, and in a narrower range $0.06<\alpha<0.1$ for the curve $\mu=17$. However, the difference between 


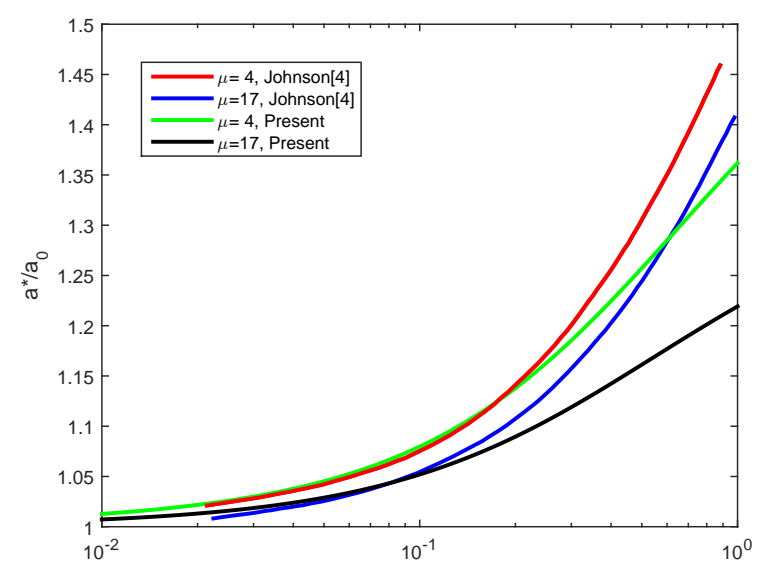

(a) $10^{-2} \leq \alpha \leq \stackrel{\alpha}{1} ; \mu=4,17$

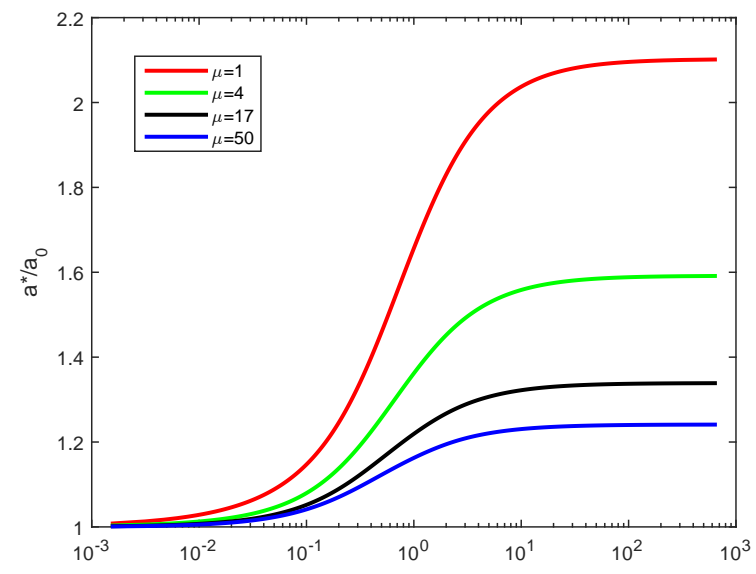

(b) $10^{-3} \leq \alpha<10^{3} ; \mu=1,4,17,50$

Figure 3.6: The ratio of the effective contact radius $a^{*}$ to the Hertzian contact radius $a_{0}$ against $\alpha$ and $\mu$ : (a) comparison of the present work with Johnson's for $\alpha<1$ and two $\mu$; (b) the present work for wider ranges of $\alpha$ and $\mu$

the present work and the referenced work becomes larger when $\alpha$ increases towards $\alpha=1$ for $\mu=4$ and 17, and when $\alpha$ decreases from 0.06 for $\mu=17$. Again, no comparison can be made for $\alpha>1$ as no corresponding results are available in the referenced work.

Similar to the ratio $p_{G}(0) / p_{0}$, the effective contact radius $a^{*}$ over the Hertzian contact radius $a$ from the current work is provided in Figure 3.6(b) for a much wider range of $\alpha\left(10^{-3}<\alpha<10^{3}\right)$ and a larger set of $\mu(=1,4,17$ and 50$)$. The figure shows a very similar behaviour as in the ratio $p_{G}(0) / p_{0}$ that the ratio $a^{*} / a$ increases towards 1 when $\alpha$ decreases to zero regardless of $\mu$, as expected; while the ratio increases with the increase of $\alpha$ but asymptotically reaches a limit value that monotonically increases with $\mu$. On the contrary, it may also be deduced from the two curves in the referenced work that when $\alpha$ increases the ratio increases in an accelerated manner.

In summary, because both maximum pressure $p_{G}(0)$ and effective contact radius $a^{*}$ in the present work agree very well with those in the referenced work for certain ranges of $\alpha$ and $\mu$, it is with high confidence that the current implementation of the GW model is correct, while the observed discrepancies might be due to some unknown reasons in the referenced work. It may also be concluded that the effect of the parameter $\mu$ for large $\alpha$ may not be secondary as claimed in [169].

\subsubsection{Effects of input parameters on pressure distributions}

Effects of the two parameters $\alpha$ and $\mu$ on the pressure distribution $p_{G}(r)$ over the entire contact area $[0, \bar{a}]$ and the effective contact radius $a^{*}$ have been extensively discussed, for instance, in $[64,169]$ and elsewhere, so thus will not be discussed in detail here.

Figure 3.7 illustrates the effective pressure distributions for three different $\alpha=0.1,1$ and 10 and four different $\mu=1,4,10$ and 17 , where the Hertzian distribution is for the smooth 


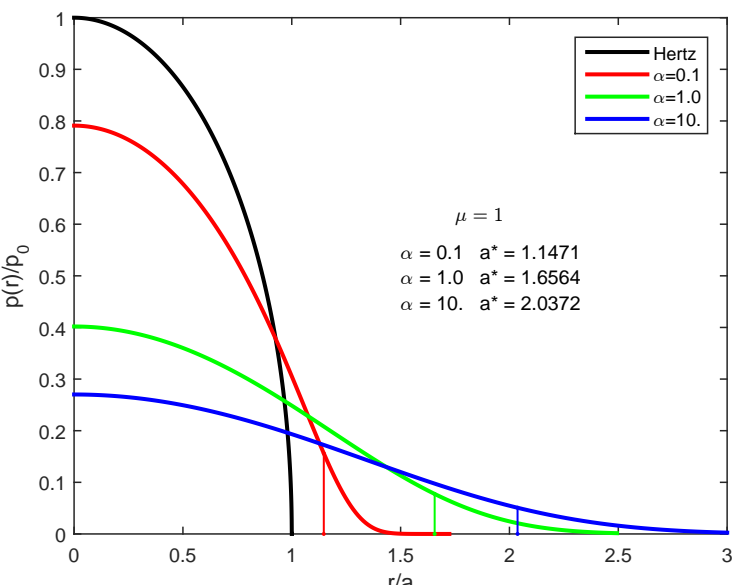

(a) $\mu=1$

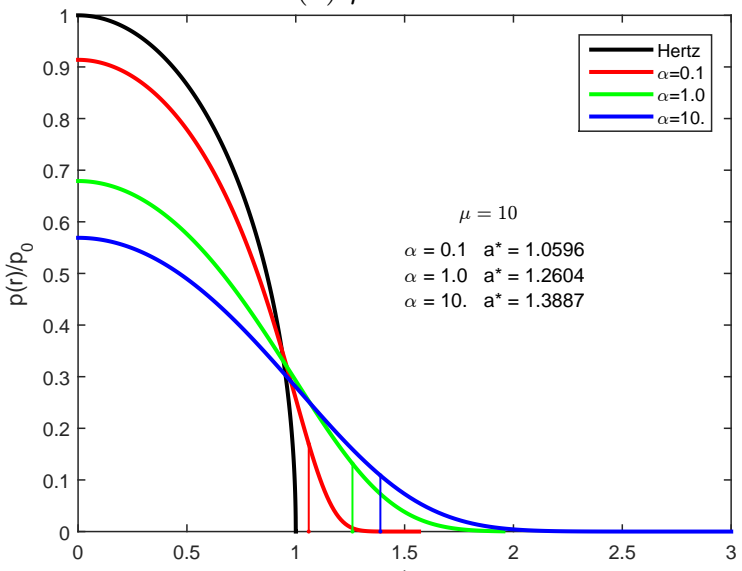

(c) $\mu=10$

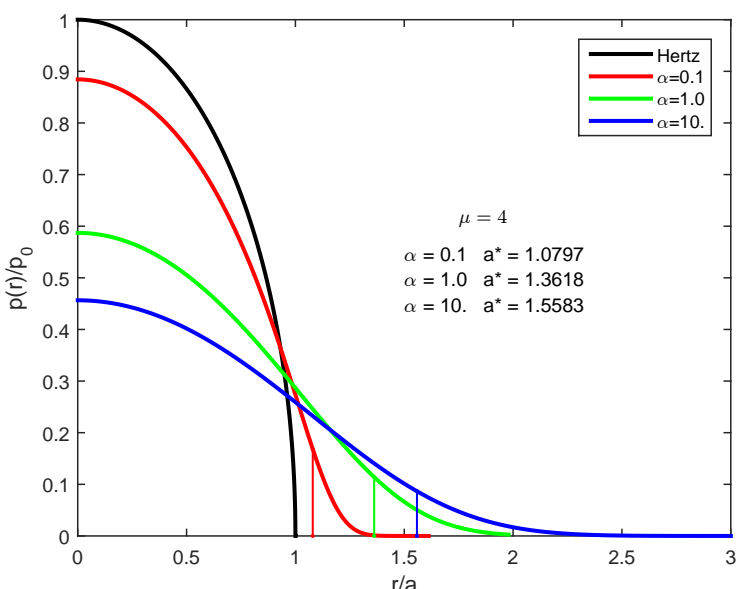

(b) $\mu=4$

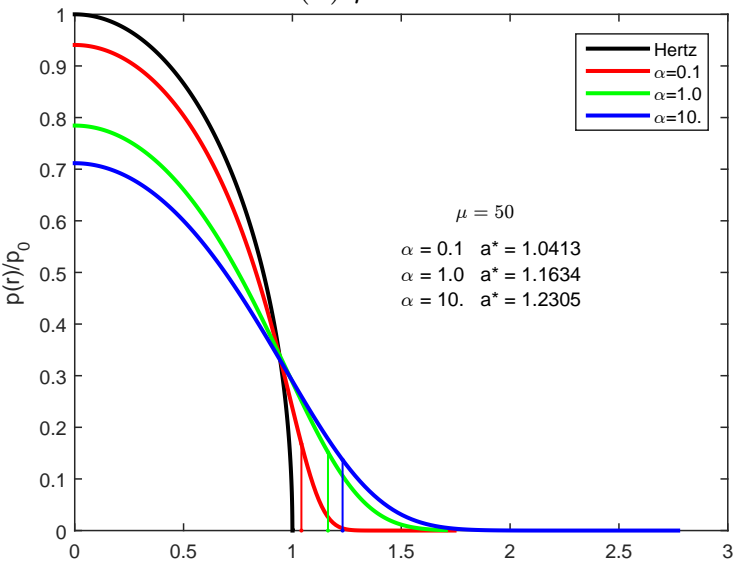

(d) $\mu=50$

Figure 3.7: Effective pressure distributions for different $\alpha$ and $\mu$ (the vertical line of each curve indicates the position of effective radius $a^{*}$ )

contact under the same contact load $P_{G}$, and the vertical line of each curve indicates the position of the corresponding effective radius $a^{*}$. As expected, the increase of $\alpha$ reduces the maximum pressure $p_{G}(0)$ but spreads the load over a greater contact area $\bar{a}$ and thereby leading to a larger effective contact radius $a^{*}$.

\subsection{Concluding remarks}

To develop a suitable normal contact law which can consider the surface roughness of particles, a Newton-Raphson based iterative solution procedure has been proposed to effectively and accurately obtain the contact pressure and deformations and the total force.

The essential components of this numerical procedure include the use of the Gaussian quadrature to evaluate three integrals, the adoption of a finite-difference approximate to the Jacobian matrix, and the determination of the coefficient $\alpha_{i j}$ and particularly the diagonal terms $\alpha_{i i}$. It reveals that the coefficients $\alpha_{i j}$ are solely determined by the number of integration points $m$ used for the chosen integration quadrature and thus can be pre-calculated. These features 
not only significantly increase the computational efficiency of the proposed numerical solution procedure, but also maintain the high accuracy of the numerical solutions.

The numerical results are obtained and validated against some existing results in the literature. Also, as given in Section 3.2.4, the classic GW model is not suitable to treat the situation with positive overlap between spheres which leads to the extension in the next chapter. 


\section{Chapter 4}

\section{Surface roughness modelling - II. Extended GW models}

\subsection{Introduction}

The above analysis in Section 3.2.4 demonstrates that the GW model cannot handle the condition when $\delta>0$, which is in accord with the general accepted conclusion that the classic GW model is better suited for light contacts with large separations [170, 171]. This difficulty is due to the assumption made in the GW model that the deformation of the interacting rough surfaces is described by the contacting asperities only, and the bulk deformation under the surface asperities has been ignored. A positive overlap between particles in DEM simulations will definitely make contribution to the contact force. Therefore, the classic GW model cannot be incorporated in the DEM modelling framework without modification.

To develop the normal contact model which can be incorporated in DEM simulation, an extended elastic GW model is presented in this chapter which can consider the positive overlap between particles. This model is further improved to allow plastic deformation at the asperities. Furthermore, under the assumptions of surface roughness described in the classical GW model, the corresponding tangential contact model and thermal conductivity model are also developed in this chapter.

\subsection{The extended elastic GW model}

To take full consideration of the positive overlap, the GW model is extended by evaluating the contributions of both the asperities and the substrate to the deformation. As the asperity deforms elastically in the classic GW model, the resulting extended model is termed the Extended Elastic GW model or the E-GW model. 


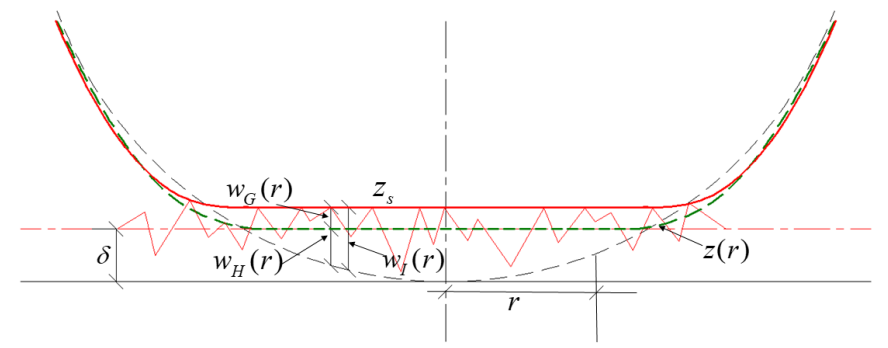

Figure 4.1: Profile of the contact between a smooth sphere and a rough surface: $\delta \geq 0$

\subsubsection{Model description}

The contact of rough particles in the discrete element modelling can be described by two steps: (a) the contact of the smooth particles with the overlap $\delta$; and (b) an additional displacement due to the surface roughness. Based on this observation, the rough (flat) surface is divided into two parts: the nominal smooth surface and the associated rough asperities, and both parts additively contribute to the deformation of the (smooth) sphere and the final contact force. As shown in Figure 4.1, the profile in green represents the deformed sphere in contact only with the smooth surface (i.e. the Hertizan part); while the profile in red represents the final deformed configuration of the sphere in contact with the rough surface. The smooth surface is taken as the datum (the red central line in Figure 4.1) which is also the mean height of the asperities.

The contact force due to the smooth part can be obtained from the Hertz law. The pressure distribution $p_{H}(r)$, the deformation distribution $w_{H}(r)$ and the total force $P_{H}(\delta)$ are given by (3.21), (3.22) and (3.23) respectively.

The additional contact force caused by the asperities is determined by the classic GW model. The profile in dashed green line can be regarded as the undeformed sphere without considering the effect of the asperities which is described by

$$
z(r)=w_{H}(r)+\frac{r^{2}}{2 R}-\delta
$$

Then the separation between the deformed sphere after contacting with asperities and the deformed sphere after contacting with the smooth surface is

$$
d(r)=w_{G}(r)+z(r)
$$

The overlap between the asperity and the dashed green line is

$$
\delta_{G}(r)=z_{s}-w_{G}(r)-z(r)
$$

Then the contact pressure distribution $p_{G}(r)$ and deformation distribution $w_{G}(r)$ can be 
expressed as

$$
\begin{aligned}
& p_{G}(r)=C \int_{d(r)}^{+\infty}\left[\delta_{G}(r)\right]^{3 / 2} \phi\left(z_{s}\right) d z_{s} \\
& w_{G}(r)=\frac{4}{\pi E} \int_{0}^{\bar{a}} \frac{t}{r+t} p_{G}(t) \mathbf{K}(k) d t
\end{aligned}
$$

The contact pressure is the sum of the Hertz pressure and the GW pressure. Thus the total pressure distribution $p(r)$ and deformation distribution $w(r)$ of the sphere can be expressed by

$$
\begin{gathered}
p(r)=p_{H}(r)+p_{G}(r) \\
w(r)=w_{H}(r)+w_{G}(r)
\end{gathered}
$$

The total contact force $P(\delta, \sigma)$ is the summation of the Hertz force $P_{H}(\delta)$ and the rough GW contribution $P_{G}(\delta, \sigma)$ defined by $(4.4)$ as

$$
P(\delta, \sigma)=P_{H}(\delta)+P_{G}(\delta, \sigma)
$$

By utilising the fact that the Hertz contribution is zero when $\delta$ is negative, the above extended GW model includes the classic case as a special case. For the rough part, $p_{G}$ can be set to be zero when $\delta<-3 \sigma$ because the probability that a summit $z_{s}$ lies in the range $[-3 \sigma,+3 \sigma]$ is $99.9 \%$.

\section{Comparison between the classic and the extended models}

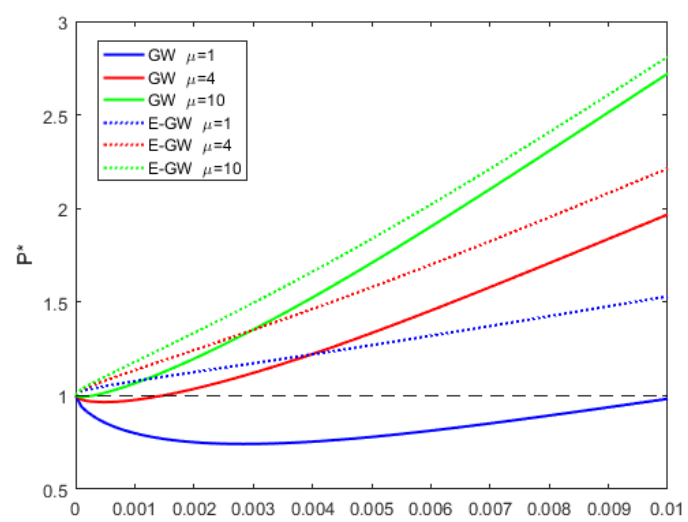

Figure 4.2: Comparison of non-dimensional total contact forces between the GW and E-GW models for different degrees of roughness

The classic GW model and the E-GW model are compared in this subsection. Unlike in the traditional tribology where the interest is mainly focused on the evolution of the separation and effective contact area under a varied normal load, the attention in the current work is concentrated on the change of the total contact force with an increasing surface roughness under the same overlap between particles. Therefore, the non-dimensional total contact 
force, the pressure distribution and the deformation distribution under the same overlap are compared between the two models.

Figure 4.2 shows the relationship between the surface roughness $\sigma$ and the non-dimensional total contact force $P^{*}\left(=P / P_{H}\right)$ with a positive overlap $\delta=0.01$. The roughness $\sigma$ increases from 0 to 0.01 and three different values, 1,4 and 10, are chosen for the non-dimensional parameter $\mu$. The rough surface is supposed to result in a larger normal contact force than the smooth surface with the same overlap, which means the non-dimension total contact force $P^{*}$ should always be greater than 1 and should also increase with the increase of the degree of roughness $\sigma$.

However, it is evident from Figure 4.2 that when $\sigma$ is approaching to zero, the non-dimensional contact forces $P^{*}$ obtained from the GW model are smaller than 1, meaning the contact forces of rough particles are smaller than those of smooth particles. This is physically incorrect and is consistent with the theoretical predication presented in Section 3.2.4. On the other hand, the extended model correctly captures the phenomenon that rougher surfaces produce larger contact forces than smoother surfaces under the same overlap.

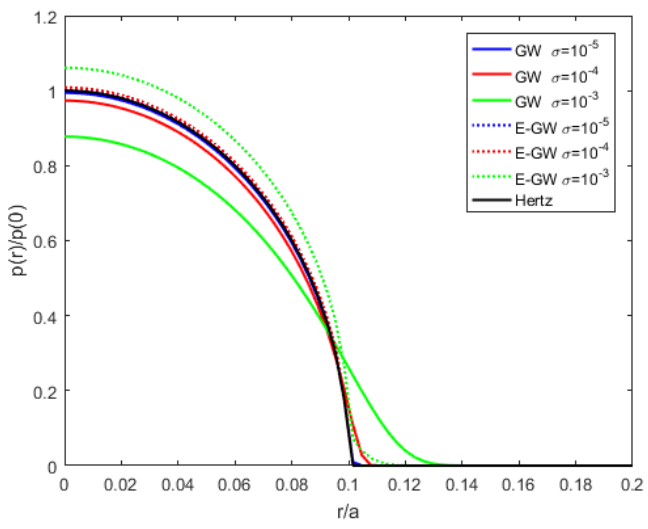

(a) Pressure distribution

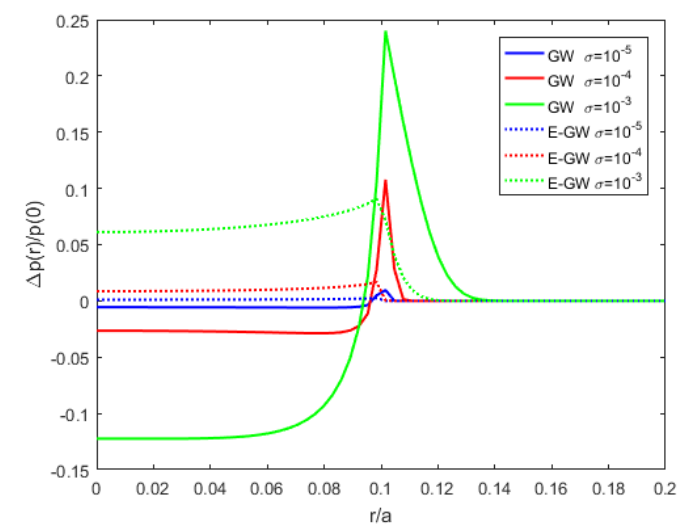

(b) Pressure difference distribution

Figure 4.3: Comparison of pressure distributions over the contact zone

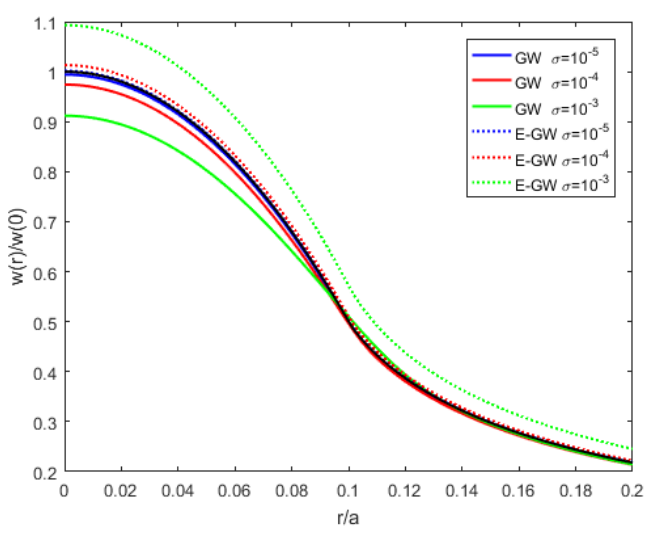

(a) Deformation distribution

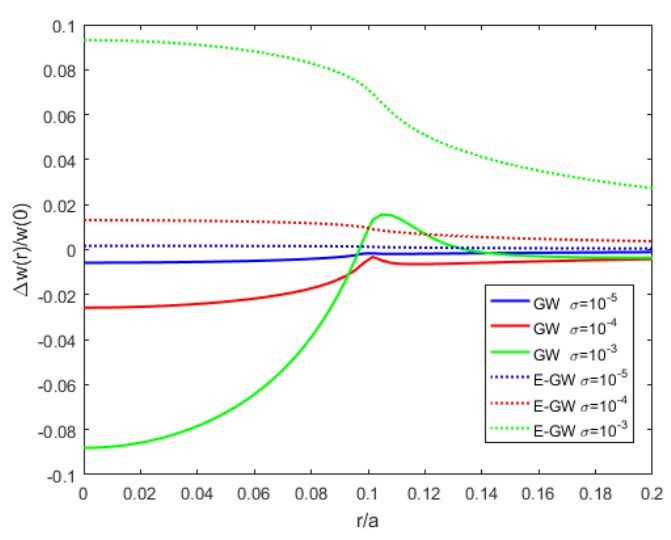

(b) Deformation difference distribution

Figure 4.4: Comparison of deformation distributions with over the contact zone 
The pressure and deformation distributions based on the classic GW model and the E-GW model are also compared and shown in Figure 4.3(a) and Figure 4.4(a) respectively. To make the comparison more apparent, the differences of these distributions with the corresponding Hertz solutions are depicted in Figure 4.3(b) and Figure 4.4(b). The results are obtained with the same positive overlap $\delta=0.01$, the same non-dimensional parameter $\mu=4$ and three different surface roughness levels $\sigma=10^{-5}, 10^{-4}$, and $10^{-3}$.

The fact that the pressure and the deformation gradually approach to the Hertz solution as $\sigma$ decreases when the surface becomes smoother can be seen for both models but in different fashions. The classic GW model achieves this from below the Hertz solution in most of the contact region which is again conforming to the theoretical analysis in Section 3.2.4. Also the surface asperities reduce the pressure and deformation in the original contact area between the sphere and the smooth surface but lead to a significantly larger effective contact area. On the contrary, the E-GW model approaches to the Hertz solution from above which reflects the fact that, as the surface becomes rougher, both pressure and deformation increase inside and outside the original contact area.

In DEM simulations, a positive overlap is defined between two smooth particles (for dry mechanical contact only), the asperities added on the smooth surface should cause additional pressure and deformation. Therefore, it can be concluded that the current extended model is more realistic for the contact situation with a positive overlap.

\subsubsection{A normal interaction law based on the E-GW model}

The aim of this work is to establish a normal contact law that can be readily used in DEM. Considering the complicity of the above extended GW model, nondimensional analysis as defined in Section 3.3 is performed to make the final formulas with a minimum number of parameters.

The $\delta$-form:

$$
\begin{aligned}
p^{*}\left(r^{*}, \alpha\right) & =p_{H}^{*}\left(r^{*}\right)+p_{G}^{*}\left(r^{*}, \alpha\right) \\
& =\frac{2 a^{*}}{\pi}\left(1-\frac{r^{* 2}}{a^{* 2}}\right)^{1 / 2}+\mu \int_{w_{G}^{*}\left(r^{*}, \alpha\right)}^{\infty}\left[z_{s}^{*}-w_{G}^{*}\left(r^{*}, \alpha\right)\right]^{3 / 2} \phi\left(z_{s}^{*}\right) d z_{s}^{*} \\
w^{*}\left(r^{*}, \alpha\right) & =w_{H}^{*}\left(r^{*}\right)+w_{G}^{*}\left(r^{*}, \alpha\right) \\
& =\left(1-\frac{r^{* 2}}{2 a^{* 2}}\right)+\frac{2}{\pi} \int_{0}^{\bar{a}^{*}} \frac{t^{*}}{t^{*}+r^{*}} p_{G}^{*}\left(t^{*}, \alpha\right) \mathbf{K}(k) d t^{*} \\
P^{*}(\alpha, \mu) & =P_{H}^{*}+P_{G}^{*}(\alpha, \mu)=1+\frac{3 \sqrt{2}}{8} \int_{0}^{\bar{a}^{*}} 2 \pi r^{*} p_{G}^{*}\left(r^{*}, \alpha\right) d r^{*}
\end{aligned}
$$


The $\sigma$-form:

$$
\begin{aligned}
p^{*}\left(r^{*}, \alpha^{\prime}\right) & =p_{H}^{*}\left(r^{*}\right)+p_{G}^{*}\left(r^{*}, \alpha^{\prime}\right) \\
& =\frac{2 a^{*}}{\pi}\left(1-\frac{r^{* 2}}{a^{* 2}}\right)^{1 / 2}+\mu \int_{w_{G}^{*}\left(r^{*}, \alpha^{\prime}\right)}^{\infty}\left[z_{s}^{*}-w_{G}^{*}\left(r^{*}, \alpha^{\prime}\right)\right]^{3 / 2} \phi\left(z_{s}^{*}\right) d z_{s}^{*} \\
w^{*}\left(r^{*}, \alpha^{\prime}\right) & =w_{H}^{*}\left(r^{*}, \alpha^{\prime}\right)+w_{G}^{*}\left(r^{*}, \alpha^{\prime}\right) \\
& =\alpha^{\prime}\left(1-\frac{r^{* 2}}{2 a^{* 2}}\right)+\frac{2}{\pi} \int_{0}^{\bar{a}^{*}} \frac{t^{*}}{t^{*}+r^{*}} p_{G}^{*}\left(t^{*}, \alpha^{\prime}\right) \mathbf{K}(k) d t^{*} \\
P^{*}\left(\alpha^{\prime}, \mu\right) & =P_{H}^{*}\left(\alpha^{\prime}\right)+P_{G}^{*}\left(\alpha^{\prime}, \mu\right)=1+\frac{3 \sqrt{2}}{8} \int_{0}^{\bar{a}^{*}} 2 \pi r^{*} p_{G}^{*}\left(r^{*}, \alpha^{\prime}\right) d r^{*}
\end{aligned}
$$

The total contact force between two rough spheres can be expressed as

$$
P(\delta, \sigma)=P_{H}(\delta) P^{*}(\alpha, \mu)=P_{H}(\sigma) P^{*}\left(\alpha^{\prime}, \mu\right)
$$

where both non-dimensional coefficients $P^{*}(\alpha, \mu)$ and $P^{*}\left(\alpha^{\prime}, \mu\right)$ will be derived in empirical form.

Interaction laws commonly used in DEM are an explicit formula in terms of the overlap and other parameters of contact features which is obviously different from the extended GW model in Equation(4.15). Due to the complicity and implicit nature of the extended GW model which cannot be implemented directly into the DEM framework, a curve-fitting procedure is conducted to obtain a normal interaction law based on numerical results.

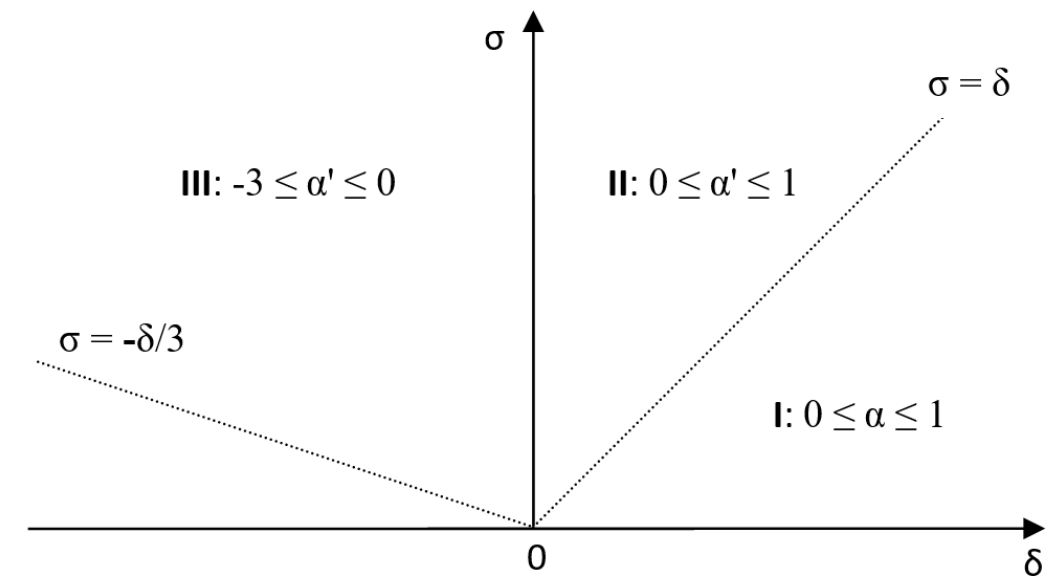

Figure 4.5: The division of the $\delta-\sigma$ plane into three cases

As $P_{H}$ and $P^{*}$ have been separated in Equation(4.15), only $P^{*}$ needs to be curve-fitted. Moreover, to avoid numerical difficulties, the range of the input parameters has been divided into three parts: Case I: $0 \leq \alpha \leq 1$; Case II: $0 \leq \alpha^{\prime} \leq 1$; and Case III: $-3 \leq \alpha^{\prime} \leq 0$, as shown in Figure 4.5.

Three explicit approximations to $P^{*}$ for the three cases, denoted as $P_{1}^{*}(\alpha, \mu), P_{2}^{*}\left(\alpha^{\prime}, \mu\right)$, $P_{3}^{*}\left(\alpha^{\prime}, \mu\right)$, are sought. The corresponding fitting results are acquired respectively with addi- 
tional requirements for the continuity conditions as

$$
P_{1}^{*}(1, \mu)=P_{2}^{*}(1, \mu) ; P_{2}^{*}(0, \mu)=P_{3}^{*}(0, \mu)
$$

The curve-fitting procedure is conducted by two steps to obtain the empirical formulas. In the first step, a limited number of values for $\mu$ are selected, and for each fixed $\mu$, a curve fitting for $P^{*}$ will be conducted. In the second step, the coefficients of the empirical functions attained will be further curve-fitted in terms of $\mu$ by interpolating functions such as cubic splines.

The first variable $\mu$ is assumed to be in the range of [1,50], and seven values of $\mu=$ $1,2,4,10,20,35,50$ are selected. For each $\mu, 200$ equally spaced values of $\alpha$ in $[0,1]$ and $1000 \alpha^{\prime}$ in $[-3,1]$ are used to generate all the curves.

\section{Empirical formula for Case I}

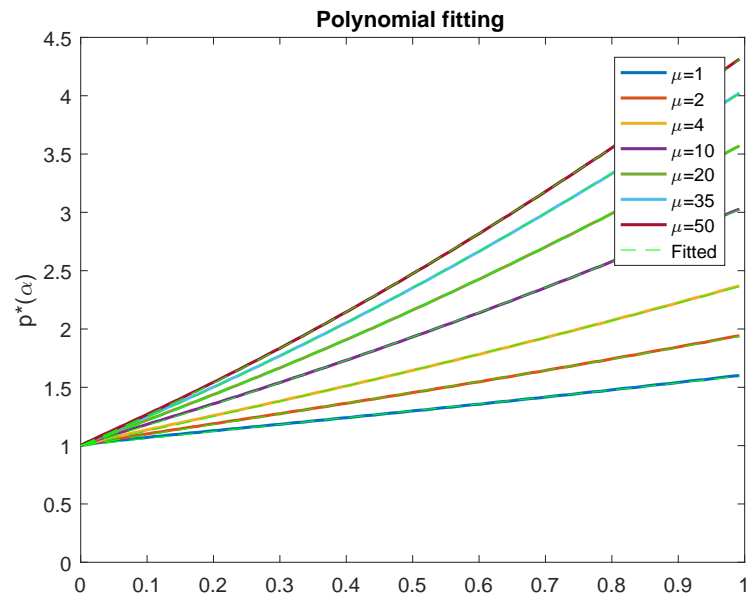

(a)

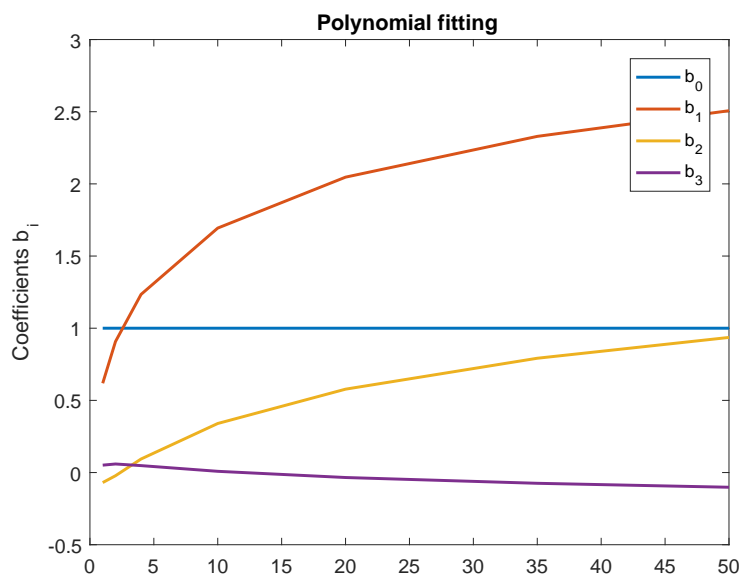

(b)

Figure 4.6: Case I - $0 \leq \alpha \leq 1$ : (a) Computed $P^{*}$ and cubic polynomial fitted curves; (b) The coefficients of the cubic polynomial

Table 4.1: Case I $(0 \leq \alpha \leq 1)$ : Coefficients of the cubic polynomial for different $\mu$

\begin{tabular}{c|ccccccc}
\hline \multirow{2}{*}{ coef. } & \multicolumn{7}{|c}{$\mu$} \\
\cline { 2 - 8 } & 1 & 2 & 4 & 10 & 20 & 35 & 50 \\
\hline$b_{0}$ & 1.0 & 1.0 & 1.0 & 1.0 & 1.0 & 1.0 & 1.0 \\
$b_{1}$ & 0.6187 & 0.9078 & 1.2343 & 1.6938 & 2.0466 & 2.3291 & 2.5069 \\
$b_{2}$ & -0.0694 & -0.0220 & 0.0938 & 0.3399 & 0.5777 & 0.7919 & 0.9361 \\
$b_{3}$ & 0.0513 & 0.0593 & 0.0481 & 0.0085 & -0.0344 & -0.0745 & -0.1018 \\
\hline \multicolumn{7}{|c}{ Fitted formula } \\
\hline$b_{0}$ & \multicolumn{7}{c}{1.0} \\
$b_{1}$ & \multicolumn{7}{|c}{$0.3484 \ln (\mu)+0.6066 \mu^{0.1642}$} \\
$b_{2}$ & \multicolumn{7}{|c}{$0.3176 \mu^{0.3782}-0.4135$} \\
$b_{3}$ & \multicolumn{7}{|c}{$0.0745 / \mu-0.1737 \mu^{0.2134}+0.2992$} \\
\hline
\end{tabular}

The numerical results of $P^{*}$ for Case I $(0 \leq \alpha \leq 1)$ are shown in Figure 4.6(a) as the solid 
lines. A cubic polynomial is chosen as the curve-fitted formula for $P_{1}^{*}(\alpha, \mu)$

$$
P_{I 1}^{*}(\alpha, \mu)=b_{0}(\mu)+b_{1}(\mu) \alpha+b_{2}(\mu) \alpha^{2}+b_{3}(\mu) \alpha^{3}
$$

which is subjected to the continuity condition (4.16).

The extended model reduces to the Hertz model when $\sigma=\alpha=0$ as

$$
P_{1}^{*}(0, \mu)=1+P_{G 1}^{*}(0, \mu)=1
$$

So

$$
b_{0}(\mu)=1
$$

Four points at $\alpha=0,1 / 3,2 / 3,1$ are selected for the interpolation function $P_{1}^{*}(\alpha, \mu)$. The fitted curves are plotted for each $\mu$ in Figure 4.6(a) by dashed lines. A very good fitting result can be observed.

The relations between four coefficients $b_{i}(i=0, \ldots, 3)$ and $\mu$ are depicted in Figure 4.6(b) and listed in Table 4.1. A nonlinear least-squares procedure is applied to acquire the fitted formula for each coefficient with $\mu$ as presented in Table 4.1.

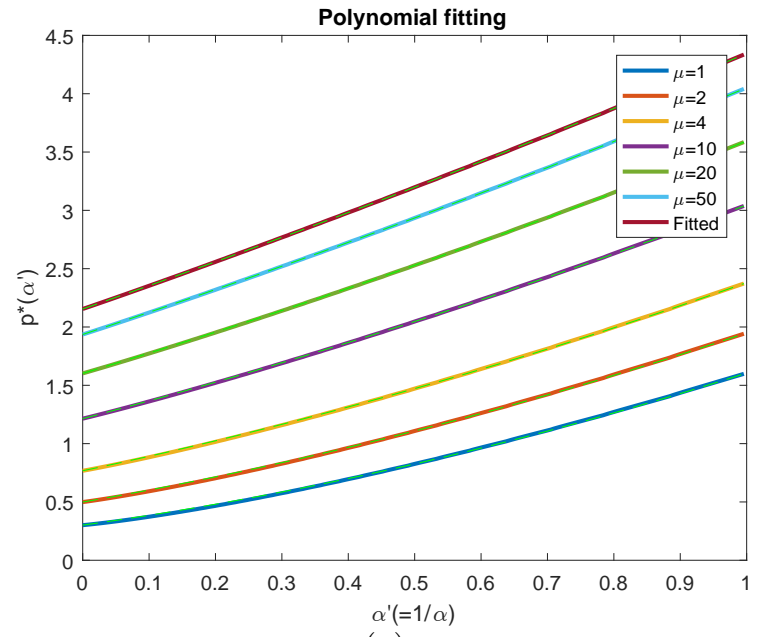

(a)

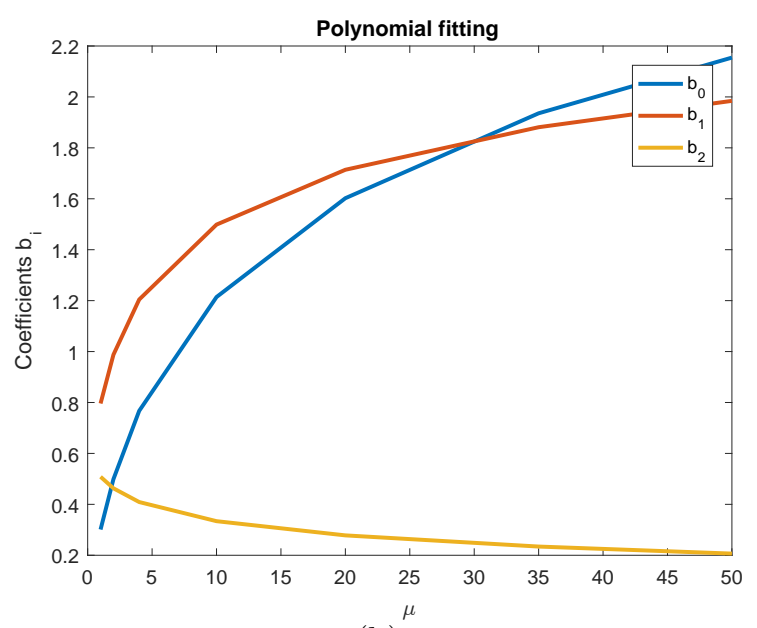

(b)

Figure 4.7: Case II $-0 \leq \alpha^{\prime} \leq 1$ : (a) Computed $P^{*}$ and quadratic polynomial fitted curves; (b) The coefficients of the quadratic polynomial

The numerical results of $P_{2}^{*}$ for Case II $0 \leq \alpha^{\prime} \leq 1$ are showed in Figure 4.7(a) as the solid lines. A quadratic polynomial is chosen as the curve-fitted formula for $P_{2}^{*}\left(\alpha^{\prime}, \mu\right)$. The continuity condition should also be satisfied in this case.

$$
P_{2}^{*}\left(\alpha^{\prime}, \mu\right)=b_{0}(\mu)+b_{1}(\mu) \alpha^{\prime}+b_{2}(\mu) \alpha^{2}
$$

$P_{2}^{*}\left(\alpha^{\prime}, \mu\right)$ is determined as the interpolation function passing through the three points at $\alpha=0,1 / 2,1$. Similar to Case I, the fitted results are presented both in Figure 4.7 and Table 4.2. It is evident that a very good fitting result has been achieved. 
Table 4.2: Case II $\left(0 \leq \alpha^{\prime} \leq 1\right)$ : Coefficients of the quadratic polynomial for different $\mu$

\begin{tabular}{c|ccccccc}
\hline \multirow{2}{*}{ coef. } & \multicolumn{7}{c}{$\mu$} \\
\cline { 2 - 7 } & 1 & 2 & 4 & 10 & 20 & 35 & 50 \\
\hline$b_{0}$ & 0.3011 & 0.4989 & 0.7672 & 1.2141 & 1.6022 & 1.9357 & 2.1544 \\
$b_{1}$ & 0.7960 & 0.9877 & 1.2044 & 1.4986 & 1.7139 & 1.8810 & 1.9845 \\
$b_{2}$ & 0.5082 & 0.4630 & 0.4090 & 0.3339 & 0.2781 & 0.2342 & 0.2066 \\
\hline & \multicolumn{7}{|c}{ Fitted formula } \\
\hline$b_{0}$ & \multicolumn{7}{|c}{$0.2284 \ln (\mu)+0.2786 \mu^{0.3913}$} \\
$b_{1}$ & \multicolumn{7}{|c}{$0.2688 \ln (\mu)+0.7873 \mu^{0.0451}$} \\
$b_{2}$ & \multicolumn{7}{|c}{$0.0778 \ln (\mu)+0.5132 \mu^{-0.0008}$} \\
\hline
\end{tabular}

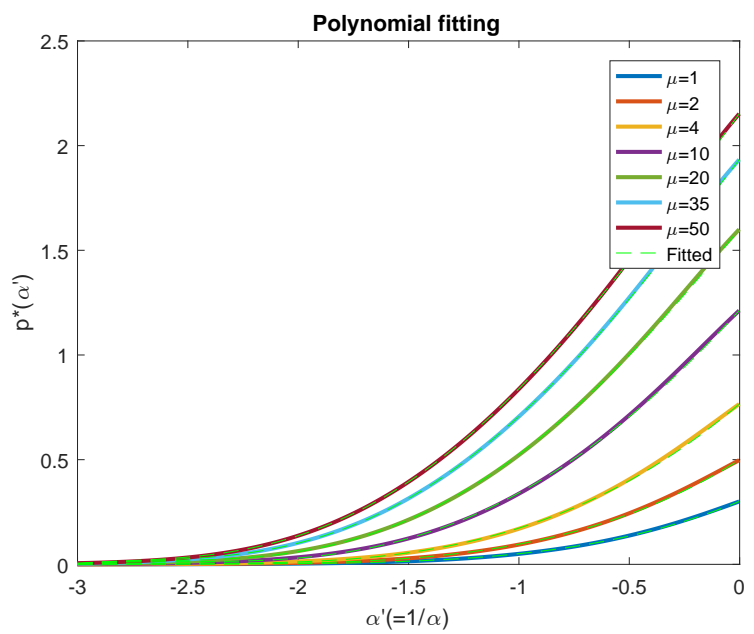

(a)

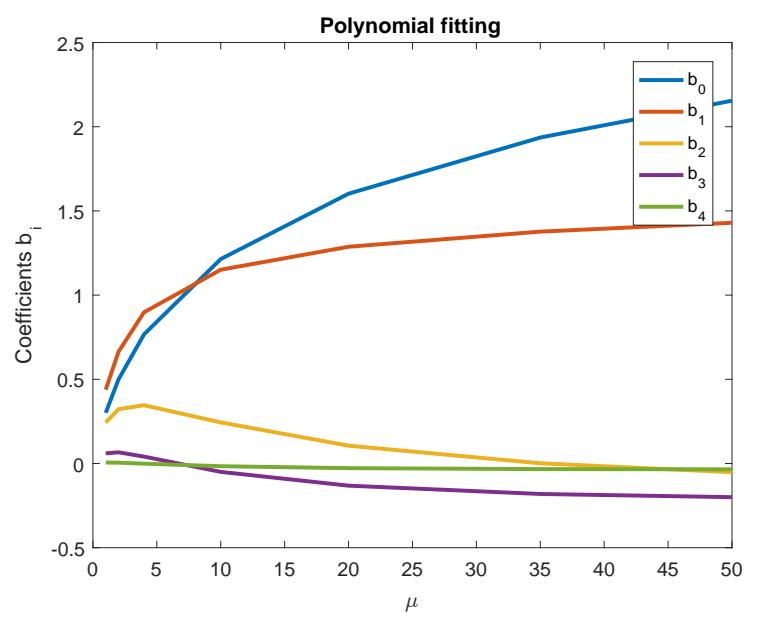

(b)

Figure 4.8: Case III - $-3 \leq \alpha^{\prime} \leq 0$ : (a) Computed $P^{*}$ and quartic polynomial fitted curves; (b) The coefficients of the quartic polynomial

\section{Empirical formula for Case III}

The numerical results of $P_{3}^{*}$ for Case III $-3 \leq \alpha^{\prime} \leq 0$ are showed in Figure 4.8(a) as the solid lines. A quartic polynomial is chosen as the curve-fitted formula for $P_{3}^{*}\left(\alpha^{\prime}, \mu\right)$. The continuity condition should also be satisfied in this case.

$$
P_{3}^{*}\left(\alpha^{\prime}, \mu\right)=b_{0}(\mu)+b_{1}(\mu) \alpha^{\prime}+b_{2}(\mu) \alpha^{2}+b_{3}(\mu) \alpha^{\prime 3}+b_{4}(\mu) \alpha^{\prime 4}
$$

$P_{3}^{*}\left(\alpha^{\prime}, \mu\right)$ is determined as the interpolation function passing through the five points at $\alpha=-3,-9 / 4,-6 / 4,-3 / 4,0$. Similar to the previous cases, the fitted results are presented both in Figure 4.8 and Table 4.3. It is evident that a very good fitting result has been achieved. In summary, the final explicit form of the normal interaction law based on the extended GW 
Table 4.3: Case III $\left(-3 \leq \alpha^{\prime} \leq 0\right)$ : Coefficients of the quartic polynomial for different $\mu$

\begin{tabular}{c|ccccccc}
\hline \multirow{2}{*}{ coef. } & \multicolumn{7}{c}{$\mu$} \\
\cline { 2 - 7 } & 1 & 2 & 4 & 10 & 20 & 35 & 50 \\
\hline$b_{0}$ & 0.3011 & 0.4989 & 0.7672 & 1.2141 & 1.6022 & 1.9357 & 2.1544 \\
$b_{1}$ & 0.6187 & 0.6638 & 0.8981 & 1.1507 & 1.2872 & 1.3770 & 1.4295 \\
$b_{2}$ & 0.2431 & 0.3227 & 0.3460 & 0.2440 & 0.1058 & 0.0012 & -0.0516 \\
$b_{3}$ & 0.0604 & 0.0665 & 0.0408 & -0.0498 & -0.1316 & -0.1810 & -0.1999 \\
$b_{4}$ & 0.0057 & 0.0047 & -0.0010 & -0.0161 & -0.0277 & -0.0333 & -0.0345 \\
\hline & \multicolumn{7}{c}{ Fitted formula } \\
\hline$b_{0}$ & \multicolumn{7}{c}{$0.2284 \ln (\mu)+0.2768 \mu^{0.3913}$} \\
$b_{1}$ & \multicolumn{7}{|c}{$-0.2862 / \mu+0.191 \ln (\mu)+0.7095$} \\
$b_{2}$ & \multicolumn{7}{|c}{$-0.2347 / \mu-0.2005 \ln (\mu)+0.1473 \ln (\mu)+0.226$} \\
$b_{3}$ & $-0.0338 / \mu-0.0245 \ln (\mu)+0.0005 \mu+0.0387$ \\
$b_{4}$ & \multicolumn{7}{c}{} \\
\hline
\end{tabular}

model can be expressed as

$$
P(\delta, \sigma, \mu)=\left\{\begin{array}{lr}
P_{H}(\delta) P_{1}^{*}(\alpha, \mu) ; & \delta \geq \sigma \\
P_{H}(\sigma) P_{2}^{*}\left(\alpha^{\prime}, \mu\right) ; & 0<\delta<\sigma \\
P_{H}(\sigma) P_{3}^{*}\left(\alpha^{\prime}, \mu\right) ; & -3 \sigma<\delta<0 \\
0 ; & \delta<-3 \sigma
\end{array}\right.
$$

\subsubsection{Numerical Illustrations of the E-GW model}

The new random normal interaction law based on the extended elastic GW model has been implemented into a DEM code to investigate the effect of surface roughness on the mechanical behaviour of a particle system. It should be noted that the material parameters used are artificially chosen, and no real surface roughness parameters are taken. The results obtained are therefore for illustration.

The material properties of the particles are: Young's modulus E=1GPa, Poisson's ratio $\nu=0.3$, density $\rho=2000 \mathrm{~kg} / \mathrm{m}^{3}$ and frictional coefficient $f=0.2$. A constant frictional coefficient is chosen here to exclusively show the influence of the roughness parameters, although the coefficient itself is entirely determined by these parameters.

Four levels of surface roughness ratios $\sigma_{r}=(0,0.001,0.005,0.01)$ are considered for the following simulations, where $\sigma_{r}=0$ represents the smooth surface. The surface roughness $\sigma$ of a particle is set to be proportional to its radius $r: \sigma=\sigma_{r} r$. The second roughness parameter $\mu$ is taken to be 10 or 50 . The first roughness parameter $\sigma$ can be regarded as the measurement of surface roughness in the normal direction which represents the height of the asperity. The second roughness parameter may be viewed as the measurement of surface roughness in the tangent direction in relation to the number and the radii of curvature of the asperities. The surface of rough particles $(r=1)$ with different roughness parameters are depicted in Figure 4.9. 


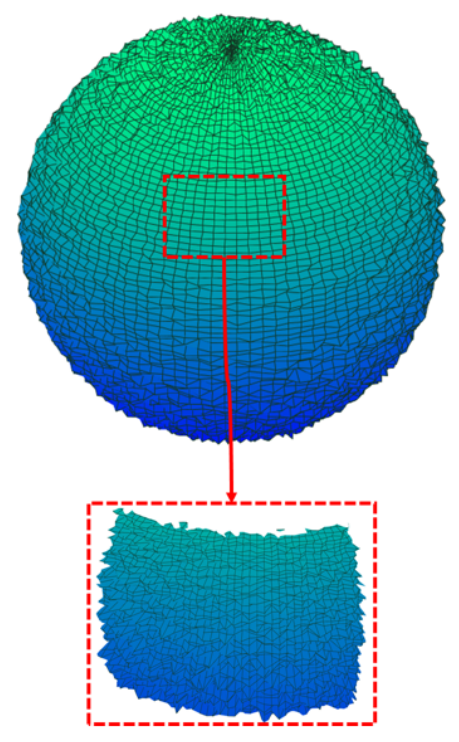

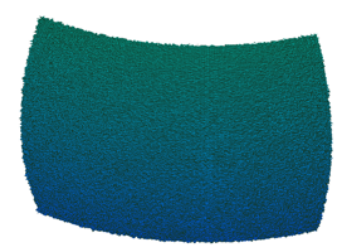

$\mu=10 \quad \sigma_{r}=0.001$
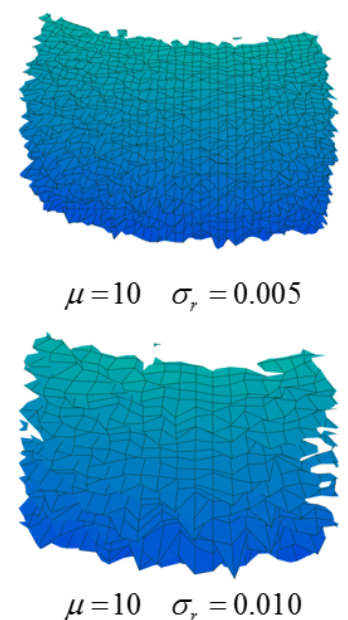

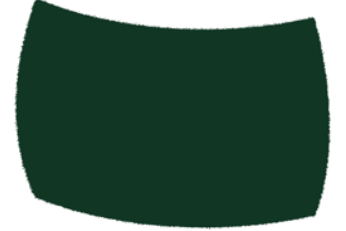

$\mu=50 \quad \sigma_{r}=0.001$

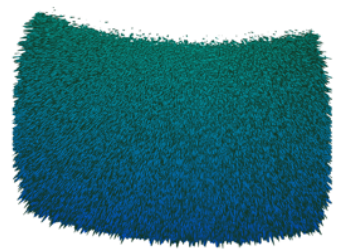

$\mu=50 \quad \sigma_{r}=0.005$

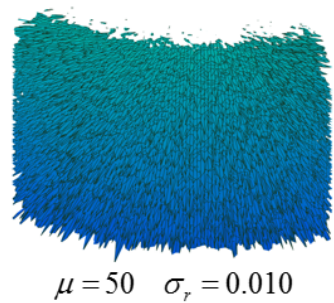

Figure 4.9: Rough surfaces of particles with different roughness parameters

In summary, seven samples with different roughness parameters have been created. The normal contact laws for a unit particle $(r=1)$ with different surface roughness parameters are showed in Figure 4.10(a), with an enlarged view in Figure 4.10(b) for overlap $\delta<0$. It is obvious that as $\sigma_{r}$ and $\mu$ increase, the value and range of the normal force increase as well. Unlike the linear or Hertz contact law which defines the relationship between the total force and the overlap by a power function with a constant exponent of 1 or 1.5.

The current normal contact law considers different contact behaviour of rough particles at different contact stages. At the initial stage of contact between two rough particles, only some asperities are in contact which corresponds to the slow growth part (Case III) of the random normal contact law. As the overlap increases from zero, the contact force between rough particles is subject to a rapid growth (Cases I and II). The random normal contact law can reflect the contact behaviour between rough particles more reasonably.

Compressive tests will be simulated below to illustrate the effect of surface roughness on the macro and micro mechanical characteristics of a particle assembly. The particle assembly is generated randomly in a cubic box of the side length $60 \mathrm{~cm}$. The particle diameters obey the Gaussian distribution with the average radius of $1 \mathrm{~cm}$ and the relative deviation of 0.25 . The total number of the particles is 14812 .

Two compressive loadings are carried out on the sample: one-dimensional compression and three-dimensional compression. For the one-dimensional compress test, the sample is first compacted to reach an initial isotropic stress of 0.5MPa. By setting the top and bottom boundaries as rigid wall, and the periodic boundary to the two lateral directions, the onedimensional compression is simulated by moving the top and bottom walls at a constant 


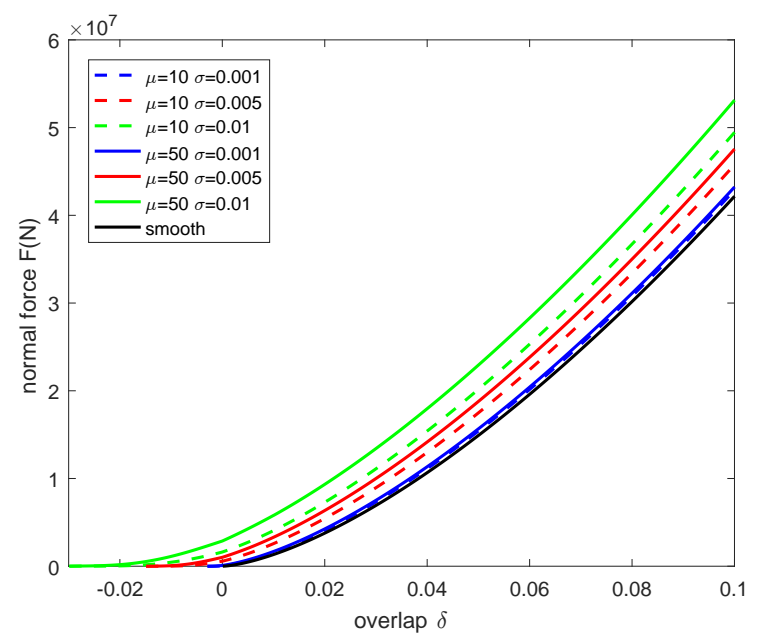

(a)

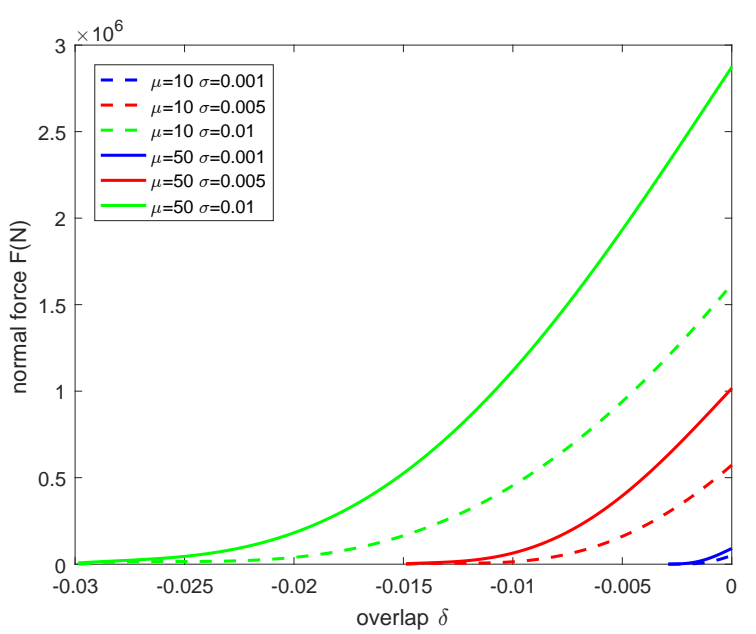

(b)

Figure 4.10: The normal force interaction laws for particles with different surface roughness parameters

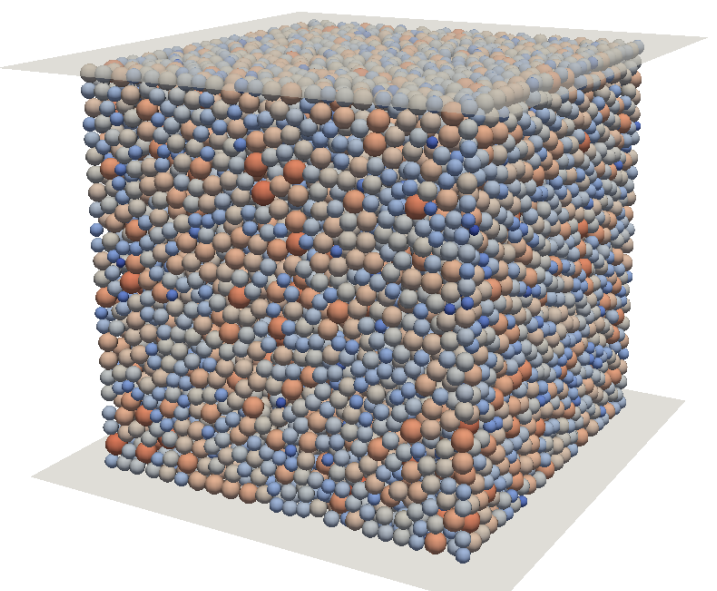

(a) One-dimensional compression

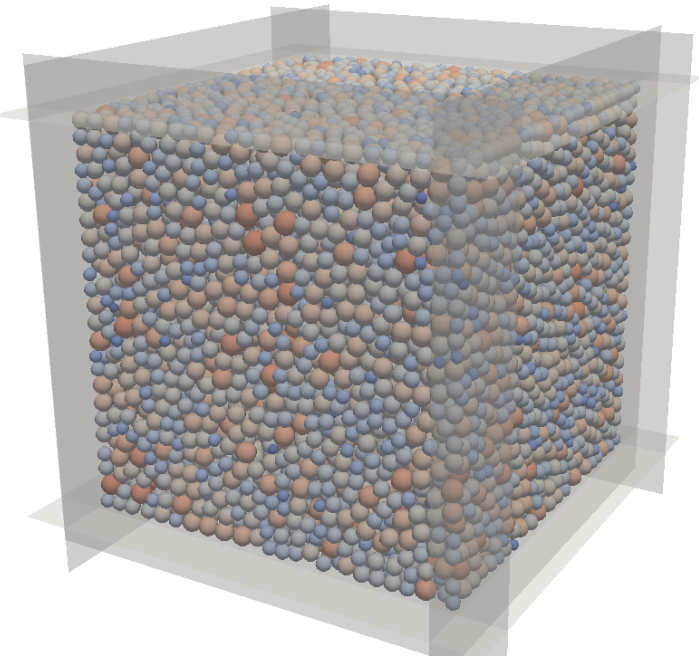

(b) Three-dimensional compression

Figure 4.11: Numerical samples

velocity in the vertical direction (Figure 4.11(a)). For the three-dimensional compress test, the initial isotropic stress is 5MPa. The sample is enclosed with rigid walls in all directions. Three-dimensional compression is simulated by moving the top and bottom walls at the same constant velocity and using a servo-control mechanism to maintain the stress on the lateral walls as 5MPa (Figure 4.11(b)).

\section{One-dimensional compression}

The initial porosities of samples with different roughness parameters with an isotropic stress state of $0.5 \mathrm{MPa}$ are displayed in Figure 4.12. It shows that as the surface roughness ratio $\sigma_{r}$ increases, the porosity almost linearly increases from 0.5670 to $0.5745(\mu=10)$ or 0.5790 $(\mu=50)$. A larger roughness parameter $\mu$ leads to a higher porosity which indicates that 


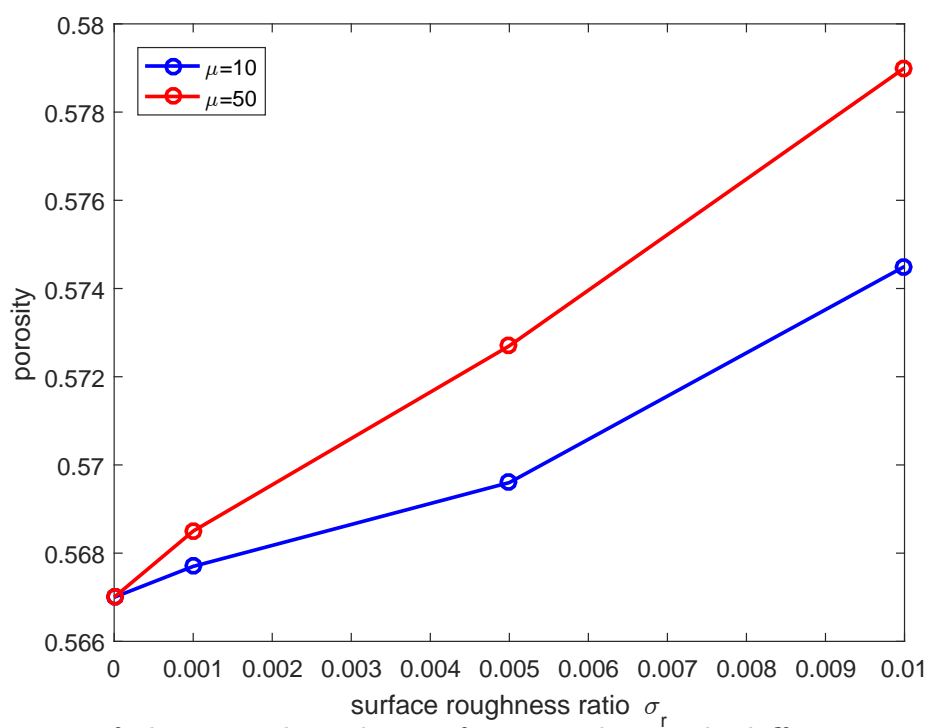

Figure 4.12: Porosities of the initial packings for samples with different roughness parameters

surface roughness makes the initial packing looser. This phenomenon can be explained by investigating the normal contacts between particles.

Table 4.4: Percentage of the number of contacts number of initial packings (One-dimensional compression)

\begin{tabular}{|c|c|c|c|c|c|c|}
\hline & \multicolumn{3}{|c|}{$\mu=10$} & \multicolumn{3}{c|}{$\mu=50$} \\
\hline Case & $\sigma_{r}=0.001$ & $\sigma_{r}=0.005$ & $\sigma_{r}=0.01$ & $\sigma_{r}=0.001$ & $\sigma_{r}=0.005$ & $\sigma_{r}=0.01$ \\
\hline I & $91.07 \%$ & $38.29 \%$ & $6.58 \%$ & $88.63 \%$ & $26.54 \%$ & $2.25 \%$ \\
II & $3.94 \%$ & $28.33 \%$ & $25.93 \%$ & $4.59 \%$ & $25.09 \%$ & $12.42 \%$ \\
III & $4.99 \%$ & $33.38 \%$ & $67.49 \%$ & $6.78 \%$ & $48.37 \%$ & $85.33 \%$ \\
\hline
\end{tabular}

Figure 4.13 depicts the normal contact links between particles. As mentioned above, the random normal contact law is divided into three cases: Case I: $\sigma \leq \delta$; Case II: $0 \leq \delta<\sigma$; Case III: $-3 \sigma \leq \delta<0$. In Figure 4.13, the contact belonging to Case I is in red, Case II in yellow and Case III in blue. It can be seen that increasing surface roughness parameters gradually increases the number of contacts in Cases II and III.

Table 4.4 lists the percentage of the number of contacts in each case for six samples with rough particles. Obviously, all the contacts in the samples of smooth particles belong to Case I as the random normal contact law reduces to the Hertz contact law. The two roughness parameters $\sigma_{r}$ and $\mu$ affect the percentage to different degrees. $\sigma_{r}$ determines the ranges of the three cases so has a more significant influence. For both $\mu=10$ and $\mu=50$, as $\sigma_{r}$ increases from 0.001 to 0.01 , the percentage of the number of contacts in Case I decreases from around $90 \%$ to less than 10\%. The percentage in Case II firstly increases then decreases as $\sigma_{r}$ increases from 0.001 to 0.05 , then to 0.01. Meanwhile, the percentage in Case III generally increases. For all $\sigma_{r}=0.001,0.05,0.01$, increasing $\mu$ increases the percentage in Case III while decreases the percentage in Cases I and II.

Figure 4.14 illustrates the normal contact distribution, average normal contact force and 


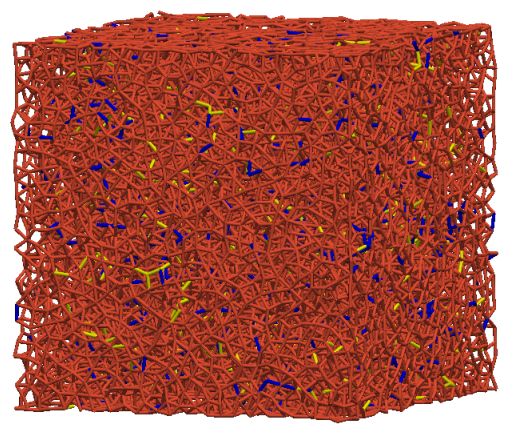

(a) $\mu=10 \quad \sigma_{r}=0.001$

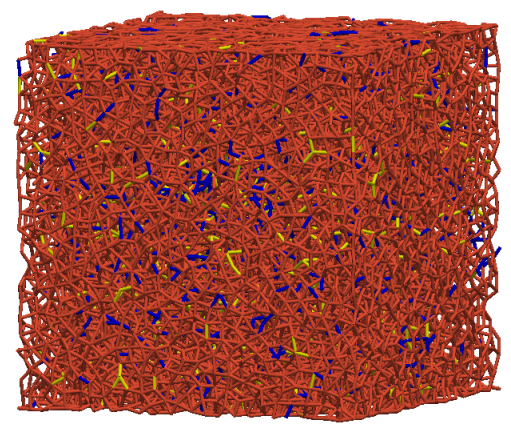

(d) $\mu=50 \quad \sigma_{r}=0.001$

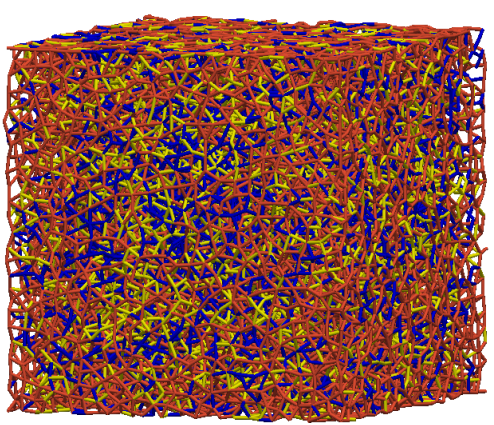

(b) $\mu=10 \quad \sigma_{r}=0.005$

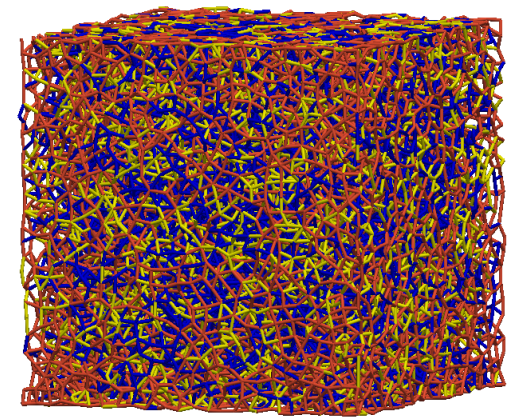

(e) $\mu=50 \quad \sigma_{r}=0.005$

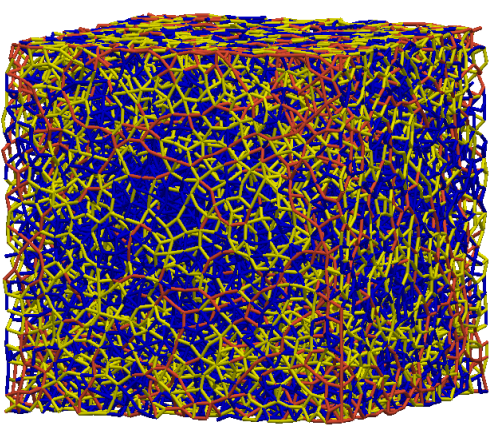

(c) $\mu=10 \quad \sigma_{r}=0.01$

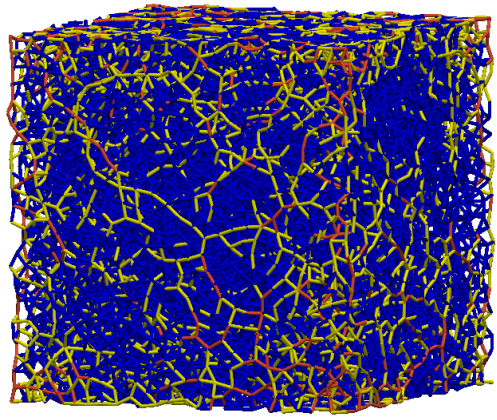

(f) $\mu=50 \quad \sigma_{r}=0.01$

Figure 4.13: Normal contact links for initial packing samples with different roughness parameters

contact force vs the overlap for different samples. The blue solid line represents the normal contact force vs overlap, the dashed red line represents the average normal force, the dashed vertical black lines divide the contacts into the three cases, and the blue histogram indicates the normal contact distribution over the overlap range. As the initial state stress for the six samples is the same, the average normal force is in the range of $1.5 \sim 1.6 \times 10^{4} \mathrm{~N}$. With the increase of $\sigma_{r}$, the average normal force decreases because the extension of the contact range leads to more normal contacts but with small values. The intersection of the average normal force and the normal contact law (red dashed line and blue solid line) determines the overlap where most contacts occur. When $\sigma_{r}=0.001$, for both $\mu$, this intersection is located within Case I, and most of the contacts occur in Case I as well. When $\sigma_{r}=0.005$, for $\mu=10$, the intersection is near the line dividing Cases II and III; the contacts in Case II (28.33\%) and Case III $(33.38 \%)$ are almost the same. While for $\mu=50$, the intersection is in Case III, making the percentage of contacts in Case III (48.37\%) two times of it in Case II $(25.09 \%)$. When $\sigma_{r}=0.01$, the intersection is in Case III and most contacts occur in Case III.

The one-dimensional compression is preformed on each sample reaching the final axial strain of 0.2. Figure 4.15 shows the compression results for seven samples. It can be seen from Figure 4.15(b) that the sample with smooth particles has the smallest coordination number. Increasing $\sigma_{r}$ will increase the coordination number while increasing $\mu$ has the opposite effect. The coordination number increases as the porosity of the sample decreases or the contact range of the normal contact law extends. The contact range is enlarged with the increase of 


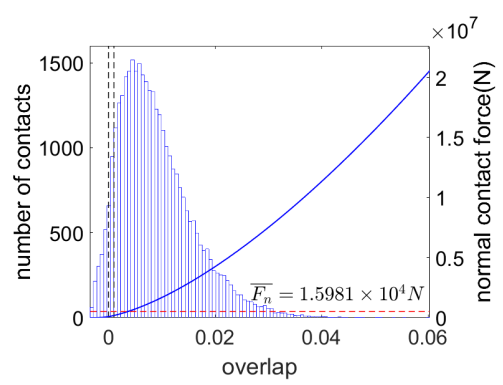

(a) $\mu=10 \quad \sigma_{r}=0.001$

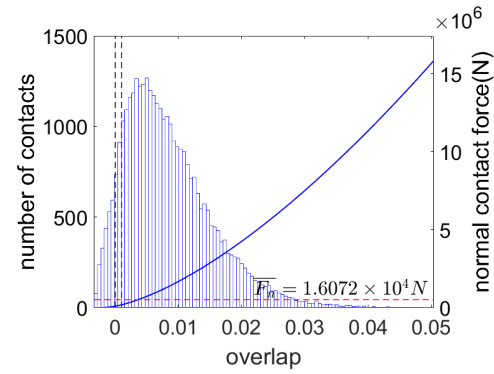

(d) $\mu=50 \quad \sigma_{r}=0.001$

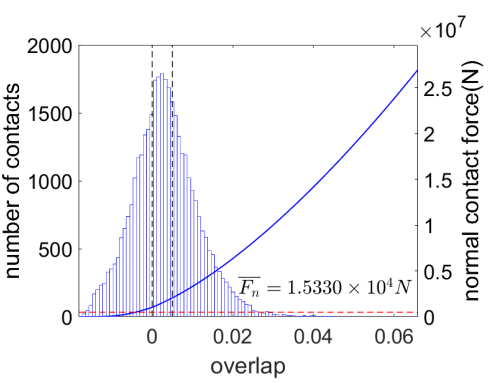

(b) $\mu=10 \quad \sigma_{r}=0.005$

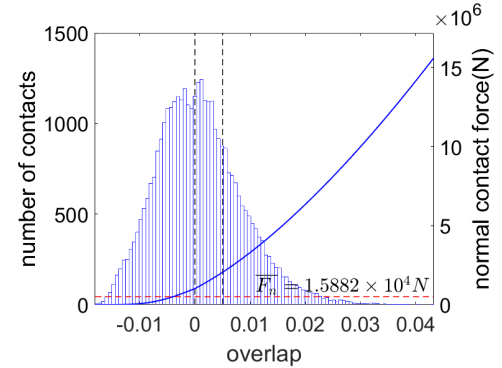

(e) $\mu=50 \quad \sigma_{r}=0.005$

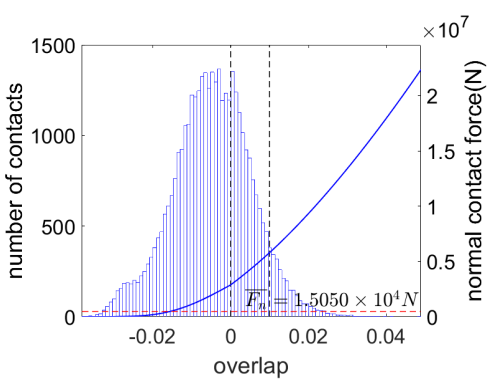

(c) $\mu=10 \quad \sigma_{r}=0.01$

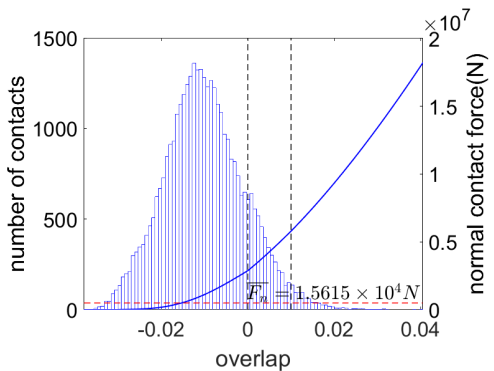

(f) $\mu=50 \quad \sigma_{r}=0.01$

Figure 4.14: Normal contact distributions and contact laws for different samples

$\sigma_{r}$ (Figure 4.10). The porosity of the sample increases with the increase of $\mu$ (Figure 4.12). Figure 4.15(a) shows the stress-strain curves, and the zoomed details of the stress-strain curves are shown in Figure 4.16, 4.17 and 4.18 for three axial strains (0.1,0.15 and 0.2), together with the corresponding contacts density distributions.

The compression stress exhibits a complicated relation with $\sigma_{r}$ and $\mu$. It can be explained by collectively considering the coordination number, the normal contact distribution and the normal contact law. Contacts between particles decide the stress state of the sample. Both the number of contacts and the corresponding forces make contributions. As roughness parameters increase, the ranges for Cases II and III become larger.

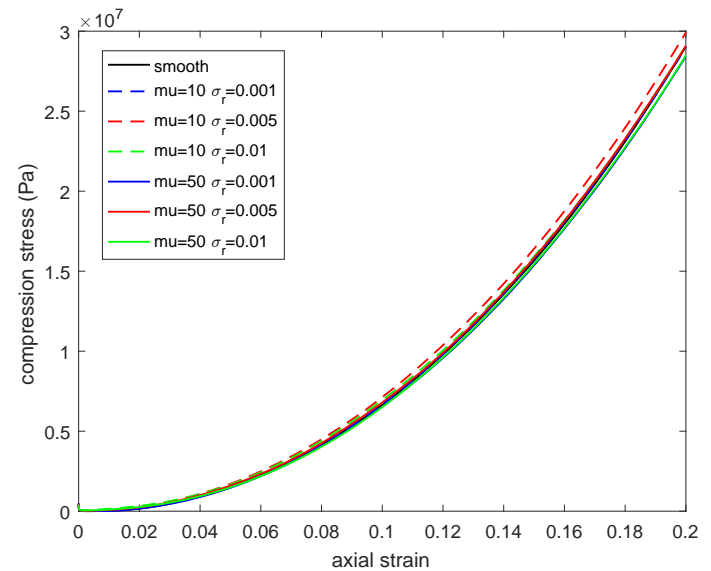

(a) Stress-strain curves

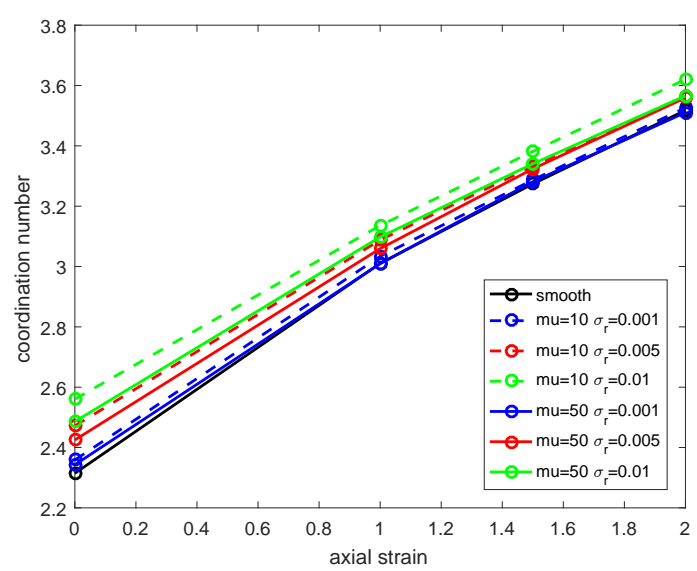

(b) Coordination number

Figure 4.15: Results of seven different samples under one-dimensional compression 


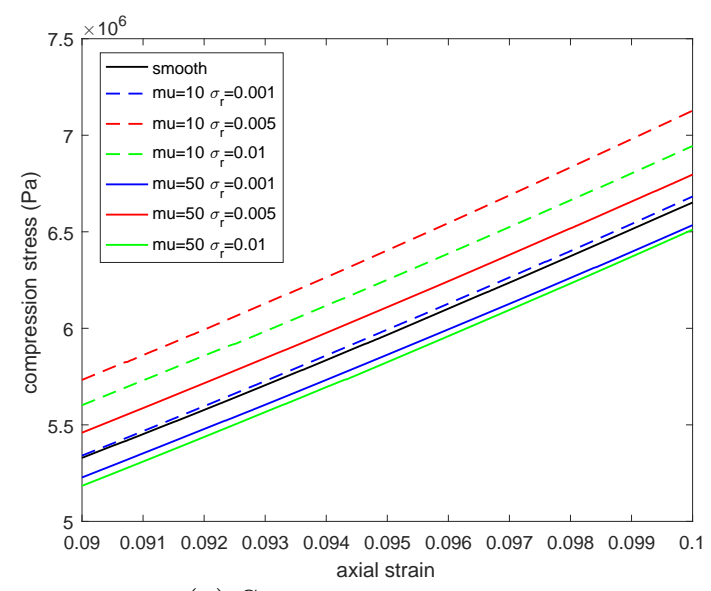

(a) Stress-strain curves

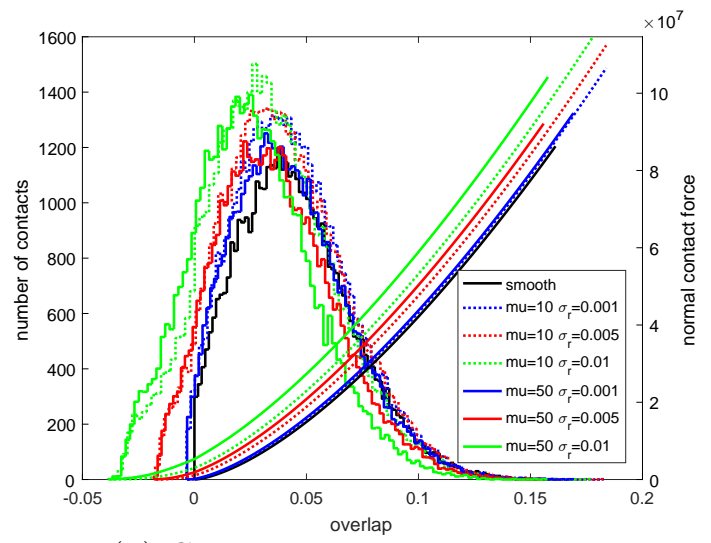

(b) Contact density distribution

Figure 4.16: Stress-strain curves and corresponding normal contact density distribution: Axial strain $=0.1$

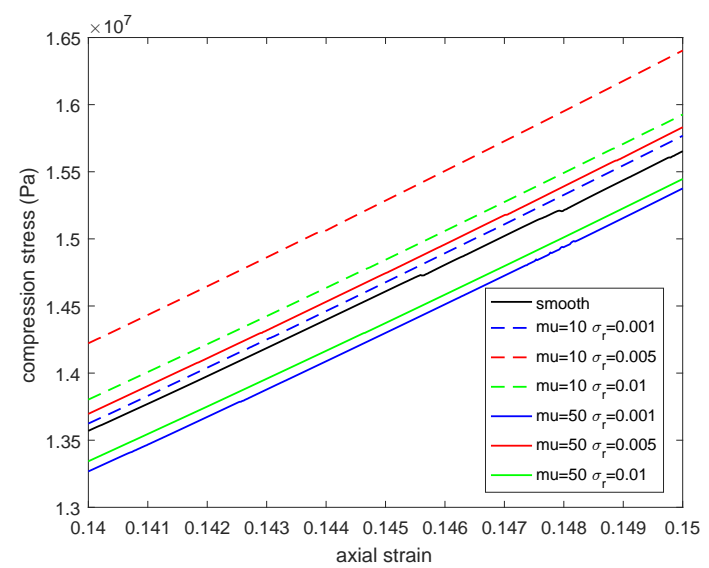

(a) Stress-strain curves

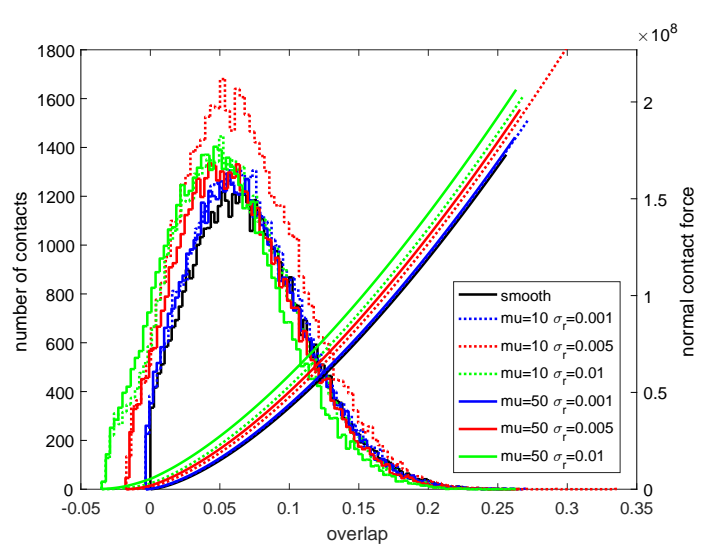

(b) Contact density distribution

Figure 4.17: Stress-strain curves and corresponding normal contact density distribution: Axial strain $=0.15$

Figure 4.16 illustrates the stress state when the axial strain is 0.1 , while the left figure is an enlarged view of Figure 4.15 for the axial strain between 0.09 and 0.1. For $\mu=10, \sigma_{r}=0.005$ produces the largest stress and $\sigma_{r}=0.001$ leads to the smallest stress. This is because increasing $\sigma_{r}$ will extend the contact range which will increase the number of contacts in Case III. A larger contact number will result in the increase of stress as shown by comparison between $\sigma_{r}=0.005$ and $\sigma=0.001$. While the contact force in Case III is very small especially for large $\sigma_{r}$, if the increase of contact number cannot offset the decrease of the normal contact force, the stress will decrease by comparison between $\sigma_{r}=0.005$ and $\sigma=0.01$. For each $\sigma_{r}$, the stress for $\mu=10$ is larger than that for $\mu=50$. This is because that the total contact number is smaller for $\mu=50$ due to a looser packing.

In Figures 4.17 and 4.18, where the axial strains are 0.15 and 0.2 respectively, the difference between the stress for $\sigma_{r}=0.001$ and $\sigma_{r}=0.01$ diminishes gradually. For $\sigma_{r}=0.01$, the stress increase is caused by more contacts in Cases I and II and fewer contacts in Case III during the compression (see Figure 4.19). For $\sigma_{r}=0.001$, small contact ranges of Cases II 


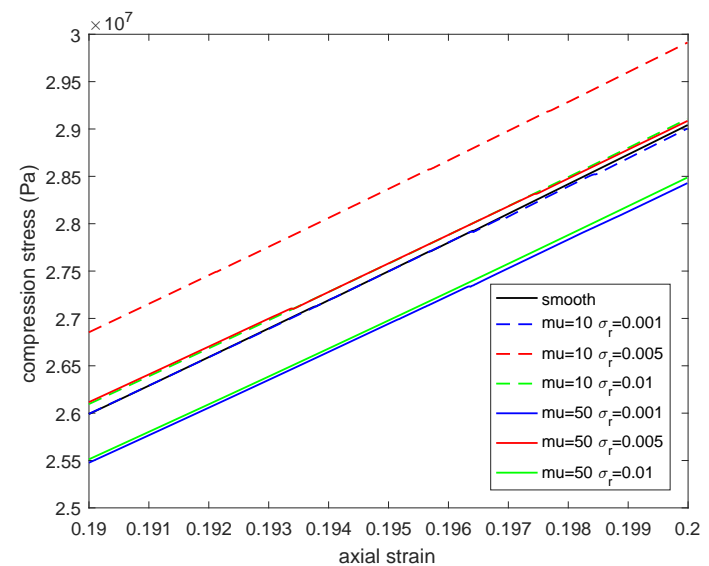

(a) Stress-strain curves

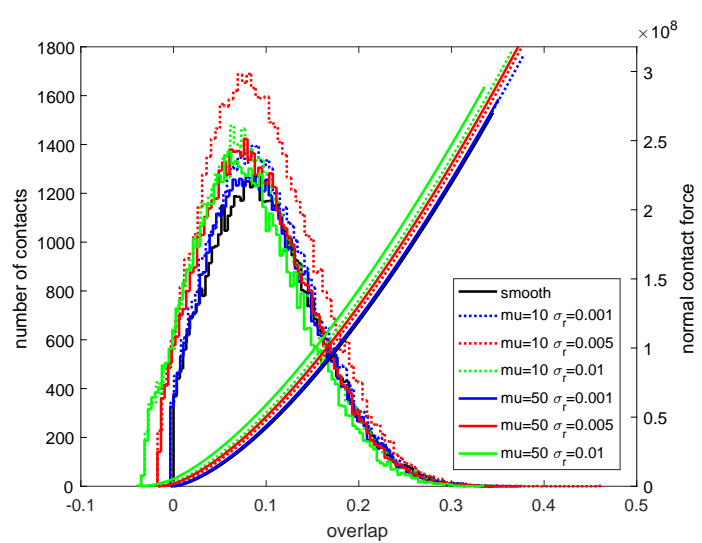

(b)Contact density distribution

Figure 4.18: Stress-strain curves and corresponding normal contact density distribution: Axial strain $=0.2$

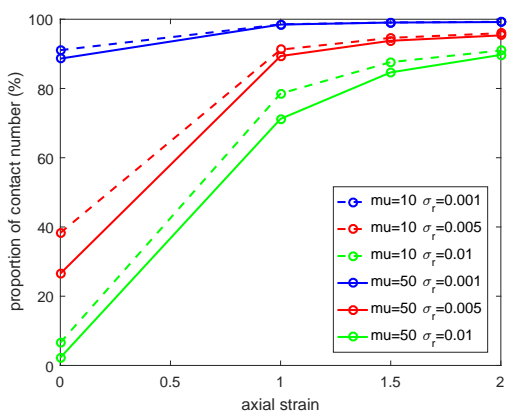

(a) Case I

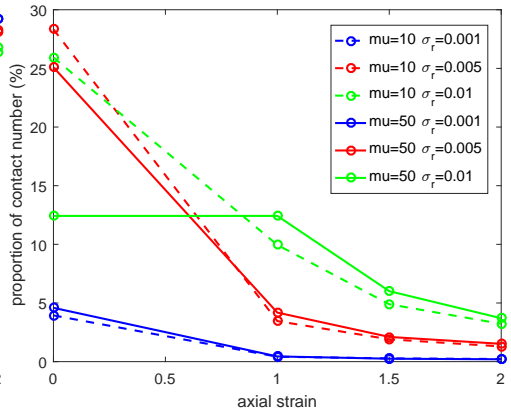

(b) Case II

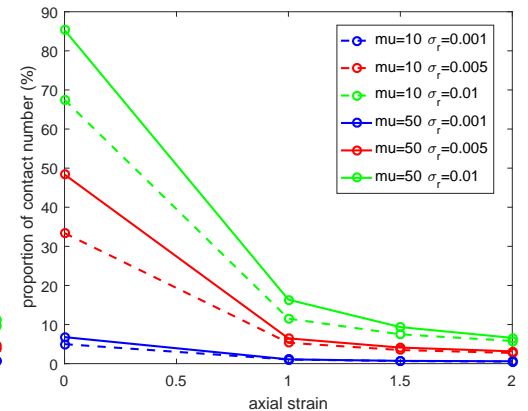

(c) Case III

Figure 4.19: Relation between percentage of the number of contacts and axial strain for three cases

and III means that most of the contacts belong to Case I. The stress of $\sigma_{r}=0.005$ is the largest. Compared to $\sigma_{r}=0.01$, smaller ranges of Cases II and III for $\sigma_{r}=0.005$ make more contacts occurring in Case I with larger normal forces. Compared to $\sigma_{r}=0.001$, the larger ranges of Case II and III for $\sigma_{r}=0.005$ gives rise to a larger contact number.

In summary, surface roughness makes the initial packing of the samples more looser. When the particle assembly is under one-dimensional compression, the sample of particles with moderate roughness parameters shows a higher strength.

\section{Three-dimensional compression}

The initial isotropic stress state for the three-dimensional compression is $5 \mathrm{MPa}$ which is ten times of that for the one-dimensional compression. Different contacts distributions can be observed from these initial packings.

Table 4.5 lists the percentage of the number of contacts in each case for all the samples of rough particles. For the smooth surface when $\sigma_{r}=0$, all the contacts belong to Case I. For all the rough samples, the percentage in Case I is dominant. Under the isotropic stress of 5MPa, 
Table 4.5: Percentage of the number of contacts of initial packings (Three-dimensional compression)

\begin{tabular}{|c|c|c|c|c|c|c|}
\hline & \multicolumn{3}{|c|}{$\mu=10$} & \multicolumn{3}{c|}{$\mu=50$} \\
\hline Case & $\sigma_{r}=0.001$ & $\sigma_{r}=0.005$ & $\sigma_{r}=0.01$ & $\sigma_{r}=0.001$ & $\sigma_{r}=0.005$ & $\sigma_{r}=0.01$ \\
\hline I & $99.0 \%$ & $93.75 \%$ & $84.21 \%$ & $98.82 \%$ & $92.38 \%$ & $76.39 \%$ \\
II & $0.33 \%$ & $2.42 \%$ & $8.32 \%$ & $0.28 \%$ & $3.08 \%$ & $11.76 \%$ \\
III & $0.67 \%$ & $3.83 \%$ & $7.47 \%$ & $0.90 \%$ & $4.54 \%$ & $11.85 \%$ \\
\hline
\end{tabular}

the packing is denser than the initial packing under the stress of 0.5MPa. More contacts are shifted to Case I.

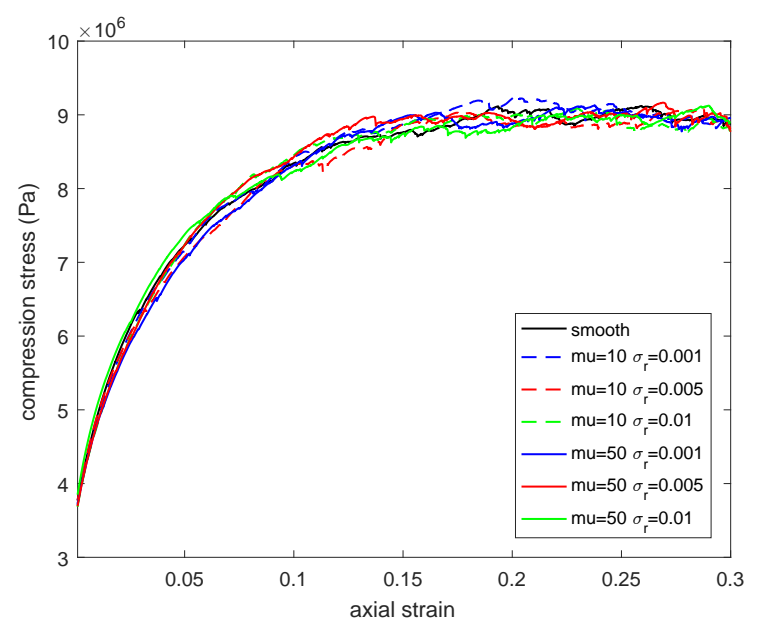

(a) Stress-strain curves

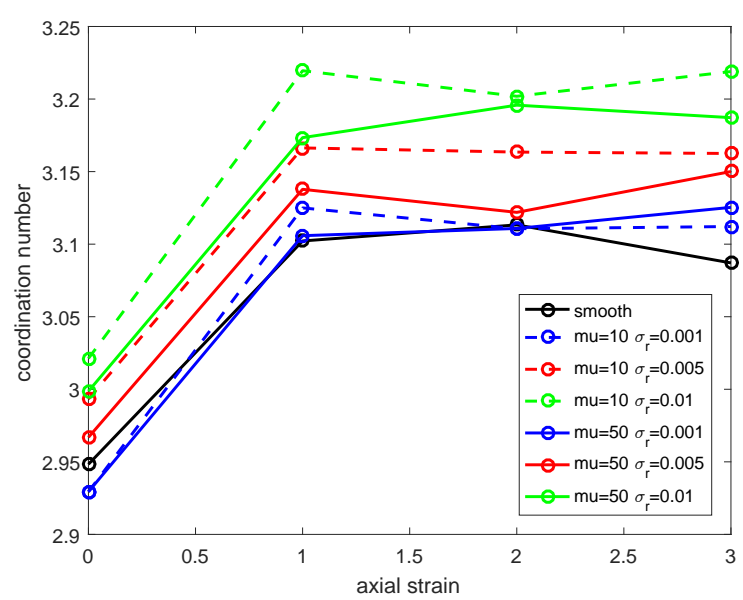

(b) Coordination number

Figure 4.20: Results of seven different samples under three-dimensional compression

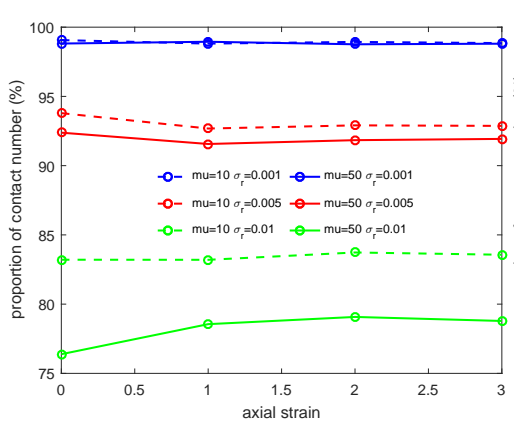

(a) Case I

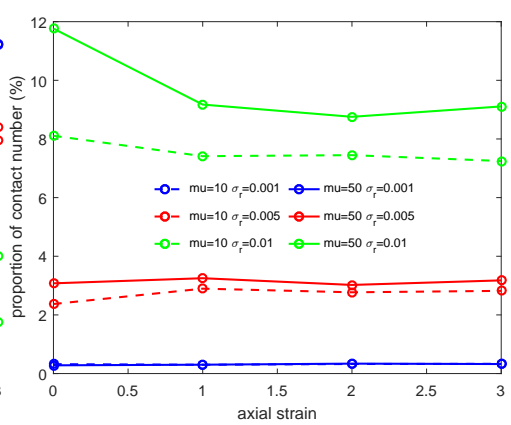

(b) Case II

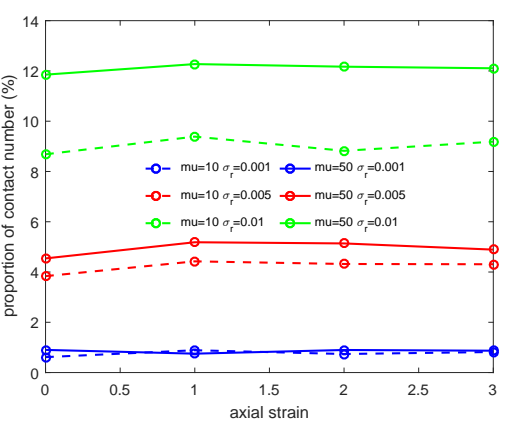

(c) Case III

Figure 4.21: Relation between percentage of the number of contacts and axial strain for three cases

The three-dimensional compression is performed on each sample reaching the final axial strain of 0.3. Figure 4.20 shows the stress-strain curves and corresponding variations of coordination number for seven samples. As the influence of roughness parameters is significant in the initial stage of the contact (Case III), the difference of the mechanical response between different samples is not obvious compared to the difference in the one-dimensional compression with the initial isotropic stress of $0.5 \mathrm{MPa}$. Two reasons result in this phenomenon. Firstly, as 
shown in Table 4.5, the large isotropic stress leads to almost the same contact distribution for different initial packings. Secondly, during the compression, the lateral compression stress is kept to be $5 \mathrm{MPa}$ which maintains a dense compaction of the sample. Under such a condition, the percentages of the number of contacts for the three contact cases keep constantly during the compression process for all the samples as shown in Figure 4.21.

In summary, the roughness parameters have a significant influence on the looser packed samples with more contacts occurring in the initial contact stage. For the dense packing, the deformation of the particles is sufficiently large which makes the influence of the surface roughness negligible.

Overall, compared to other properties of particles such as Young's modulus, Poisson's ratio and density, the asperity characteristics play a secondary role in the macro property of the particle systems. This is the main reason that the Hertz model governs the macro stress/strain curves. The influence of the surface roughness cannot be neglected, however, especially for a loosely packed particle system. The influence caused by the roughness characteristics on the packing under an initial isotropic stress of $0.5 \mathrm{MPa}$ is more significant than on the packing under the initial isotropic stress of $5 \mathrm{MPa}$. For both particle systems considered, the discrepancies caused by the roughness are more significant at the initial compressive loading stage.

\subsection{The extended elastic-plastic GW model}

When a positive overlap exists between two rough particles, the asperities may undergo substantial deformation. Therefore, the major assumption made in the classic GW model that the asperity deforms elastically may no longer hold in this case. It is thus necessary to consider the plastic deformation of asperities in the extended model.

\subsubsection{Plastic contact model}

A number of plastic contact models have been developed both for the contact problem of rough surfaces and for the single contact between two smooth spheres. These plastic contact models can be classified into two categories: analytical [170, 175-177] and semi-analytical [171, 178180]. Both categories have their own advantages and disadvantages.

The analytical contact models are based on the general physical reality that materials can exhibit different deformation behaviours during the contacting process which can be summarised into different regimes. The earlier work only contains two regimes: elastic and plastic. While the three regimes model (elastic, elasto-plastic, and fully plastic) are now more accepted. The critical overlap is proposed to indicate the inception of each regime which can be defined by the material properties. For the elastic regime, the force-displacement relationship is based on the Hertz model obeying a power law with the exponent of 1.5. For the plastic regime, a linear law is used for the force-displacement relationship. For the transition regime from elastic to plastic, the power law for the elastic regime and the linear law for 


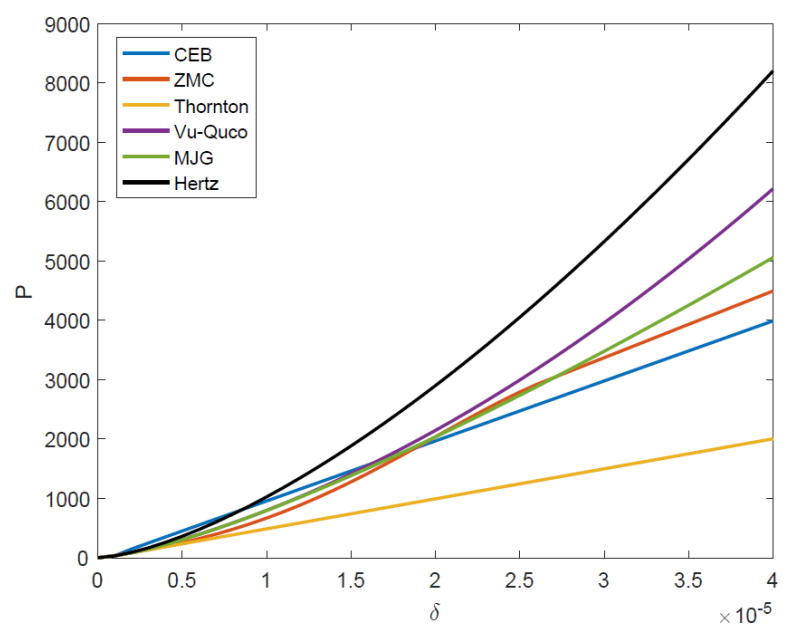

Figure 4.22: Force displacement relationships based on different contact models

the plastic regime are joined by some mathematical methods. The analytical models can be readily applied to simulate different materials while the simple assumptions used in these models make it almost impossible to fit experiments perfectly.

The semi-analytical models are based on experiments or numerical simulations which can predict the deformation behaviour of specific materials and geometries [181, 182], but the parameters need to be re-calibrated by either time-consuming experiments or numerical calculations for each material to be simulated.

Table 4.6: Parameters used in the contact models

\begin{tabular}{c|c|c|c|c|c|c}
\hline Variable & $\begin{array}{c}\text { Radius } \\
(\mathrm{m})\end{array}$ & $\begin{array}{c}\text { Young's } \\
\text { modulus } \\
(\mathrm{GPa})\end{array}$ & $\begin{array}{c}\text { Poisson's } \\
\text { ratio }\end{array}$ & $\begin{array}{c}\text { Hardness } \\
\text { coefficient }\end{array}$ & $\begin{array}{c}\text { Yield } \\
\text { stress } \\
(\mathrm{MPa})\end{array}$ & $\begin{array}{c}\text { Yield force } \\
\text { coefficient } \\
\sigma_{y}\end{array}$ \\
\hline Value & 0.1 & 76.923 & 0.3 & 0.6 & 100 & $A_{Y}$ \\
\hline
\end{tabular}

To observe the deformation behaviour described by different elasto-plastic models, the force displacement relationships for six contact models, denoted as CEB [170], ZMC [175], Thornton [176], Vu-Quoc [183], MJG [184] and Hertz respectively, are depicted in Figure 4.22 for illustrative purpose. The material and geometric parameters used to generate the curves are taken from [183], and listed in Table 4.6. Note that the Vu-Quoc model is only valid for the specific material and geometry.

The CEB, ZMC and Thornton models are analytical contact models, while the Vu-Quoc and MJG models are semi-analytical. The CEB and Thornton models contain two deformation regimes: elastic and plastic. As shown in Figure 4.22, the Thornton model predicts a much smaller contact force than the other four elasto-plastic contact models. In the CEB model, the force is even larger than that obtained from the Hertz model at the initial part of the plastic regime. Compared to the CEB and Thornton models, the ZMC model with three deformation regimes are closer to the semi-analytical models (Vu-Quoc and MJG). 


\subsubsection{Model description}

To introduce an elasto-plastic contact model for the contact between rough surfaces, the integral domain of the pressure distribution (Equation (3.15)) needs to be divided into different regions according to different critical overlaps. The contact forces between the asperities and the sphere are evaluated by the corresponding force-displacement laws. No elasto-plastic contact model is, however, widely accepted as the best choice at the moment. Considering both generality and simplicity of the formulas, an analytical elasto-plastic model is desired to be applied into our extended GW model. The resulting contact model is termed as the Extended Elasto-plastic GW (EP-GW) model. In the current work, the ZMC model [175] is adopted as it can predict the deformation behaviour more accurately compared to the other analytical models.

In the ZMC model, the three deformation regimes are determined by two critical overlaps, $\delta_{e p}$ and $\delta_{p}$. The first elasto-plastic critical overlap $\delta_{e p}$ is defined at the point when the mean contact pressure $p_{a}$ reaches $K H$ and elasto-plastic deformation occurs, which yields

$$
\delta_{e p}=\left(\frac{3 \pi K H}{4 E}\right)^{2} \beta
$$

where $H$ and $K$ are respectively the hardness and hardness coefficient of the material concerned. The second plastic critical overlap $\delta_{p}$ is defined at the point when the mean contact pressure $p_{a}$ reaches $H$ at which fully plastic deformation occurs. There is no theoretical solution available for $\delta_{p}$, however. Based on experimental results and a simple analysis, the following relation is suggested in [175]

$$
\delta_{p} \geq 54 \delta_{e p}
$$

When $\delta<\delta_{e p}$, the asperity deforms elastically. The mean contact pressure $p_{a \_e}$ and the contact area $A_{e}$ are obtained from the Hertz theory

$$
p_{a \_e}=\frac{4 E}{3 \pi} \sqrt{\frac{\delta}{\beta}} ; \quad A_{e}=\pi \beta \delta
$$

When $\delta>\delta_{p}$, the asperity deforms fully plastically. The mean contact pressure remains constant at $H$. The contact area, according to Abbott and Firestone [98], is equal to the geometrical intersection of the flat surface with the original undeformed profile of the asperity. Thus

$$
p_{a \_p}=H ; \quad A_{p}=2 \pi \beta \delta
$$

When $\delta_{e p}<\delta<\delta_{p}$, the asperity deforms elasto-plastically. The mean contact pressure $p_{a \_e p}$ and the contact area $A_{e p}$ as functions of the overlap $\delta$ become complex. The relation between $p_{a \_e p}$ and $\delta$ is derived based on the results from Francis [185] which can be characterised by a logarithmic function. Further considering the continuity of the mean pressure at the point of $\delta=\delta_{e p}\left(p_{a}=K H\right)$ and $\delta=\delta_{p}\left(p_{a}=H\right)$, the mean contact pressure in the elasto-plastic 
regime is given by

$$
p_{a \_e p}=H\left[1-(1-K) \frac{\ln \left(\delta_{p} / \delta\right)}{\ln \left(\delta_{p} / \delta_{e p}\right)}\right]
$$

The relation between the contact area $A_{e p}$ and the overlap $\delta$ is derived by joining the expressions for $A_{e}=\pi \beta w$ and $A_{p}=2 \pi \beta w$ smoothly using a cubic polynomial formula.

$$
A_{e p}=\pi \beta \delta\left[1+3 \lambda_{e p}^{2}(\delta)-2 \lambda_{e p}^{3}(\delta)\right]
$$

where

$$
\lambda_{e p}(\delta)=\frac{\delta-\delta_{e p}}{\delta_{p}-\delta_{e p}} ; \quad \lambda_{e p}\left(\delta_{e p}\right)=0 ; \quad \lambda_{e p}\left(\delta_{p}\right)=1
$$

Hence, the contact force of an asperity and the smooth sphere can be expressed as a function of the overlap $\delta$ by

$$
f(\delta)=p_{a} A=\left\{\begin{array}{lr}
\frac{4}{3} E \beta^{1 / 2} \delta^{3 / 2} ; & \delta \leq \delta_{e p} \\
\pi H \beta\left[1-(1-K) D_{1}(\delta)\right] D_{2}(\delta) ; & \delta_{e p}<\delta \leq \delta_{p} \\
2 \pi H \beta \delta ; & \delta>\delta_{p}
\end{array}\right.
$$

where

$$
\begin{aligned}
D_{1}(\delta) & =\frac{\ln \left(\delta_{p} / \delta\right)}{\ln \left(\delta_{p} / \delta_{e p}\right)} \\
D_{2}(\delta) & =\left[1+3 \lambda_{e p}^{2}(\delta)-2 \lambda_{e p}^{3}(\delta)\right] \delta
\end{aligned}
$$

Then, similar to to (3.15), the pressure distribution $p_{G}(r)$ after considering the whole rough surface can be expressed as

$$
p_{G}(r)=N \int_{d(r)}^{+\infty} f\left(z_{s}-d(r)\right) \phi\left(z_{s}\right) d z_{s}
$$

Or more explicitly

$$
\begin{aligned}
p_{G}(r) & =C \int_{d(r)}^{d(r)+\delta_{e p}}\left[\delta_{G}(r)\right]^{3 / 2} \phi\left(z_{s}\right) d z_{s} \\
& +\frac{3 C \sigma^{1 / 2}}{2 K \psi}\left\{\int_{d(r)+\delta_{e p}}^{d(r)+\delta_{p}}\left[1-(1-K) D_{1}\left(\delta_{G}(r)\right)\right] D_{2}\left(\delta_{G}(r)\right) \phi\left(z_{s}\right) d z_{s}\right. \\
& \left.+\int_{d(r)+\delta_{p}}^{+\infty} \delta_{G}(r) \phi\left(z_{s}\right) d z_{s}\right\}
\end{aligned}
$$

where $\psi$ is the plastic index defined by Greenwood and Williamson [64] as

$$
\psi=\left(\delta_{e p} / \sigma\right)^{-1 / 2}
$$


The critical overlap $\delta_{e p}$ can be expressed as

$$
\delta_{e p}=\frac{\sigma}{\psi^{2}}
$$

\subsubsection{Effects of plastic parameters}

Now this EP-GW model can consider the plastic deformation of the asperities by introducing two more parameters, the plastic index $\psi$ and the hardness coefficient $K$, compared to the E-GW model. The EP-GW model recovers the E-GW model when $\psi=0$ as $\delta_{e p}=\infty$.

The Hertz, E-GW and EP-GW models are compared below. Only the effects of the plastic parameters $\psi$ and $K$ are investigated here. The other parameters are fixed and have the same values for all the models: $\delta=0.01, \sigma=0.001, \mu=4$. The numerical solution is same to the one described in Section 3.4.

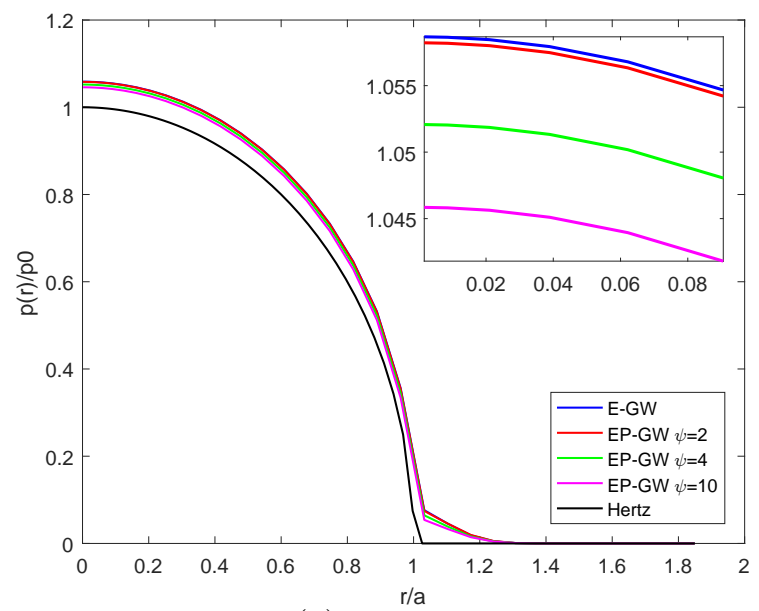

(a) $K=0.6$

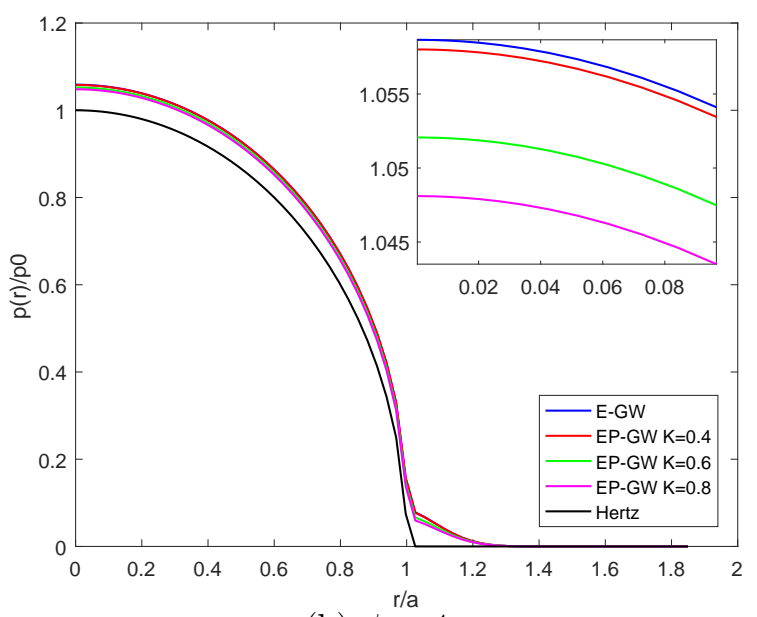

(b) $\psi \stackrel{1 / a}{=} 4$

Figure 4.23: Comparisons of the pressure distribution between different contact models

Figure 4.23(a) illustrates the pressure distribution for three different values of $\psi=2,4$ and 10 but the same value of $K=0.6$. As can be seen, the pressure calculated from the E-GW model (blue line) has the largest value as expected, which reflects the elastic effects of the surface roughness. The increase of the plastic index reduces the pressure increased by the surface roughness as more asperities deform plastically. Figure 4.23(b) illustrates the pressure distribution for three different values of $K=0.4,0.6$ and 0.8 but a fixed value of $\psi=4$. Similar results can be observed that the increase of $K$ reduces the pressure increased by the surface roughness.

The force-displacement relationship is the most interested issue when a rough surface contact model is applied to the DEM simulation. The relationships based on different models for a particularly given set of parameter values are illustrated in Figure 4.24. As expected, the increase of both $\psi$ and $K$ reduces the normal load increased by the surface roughness. Note, however, that the underlay smooth particle contact is still assumed linearly elastic. 


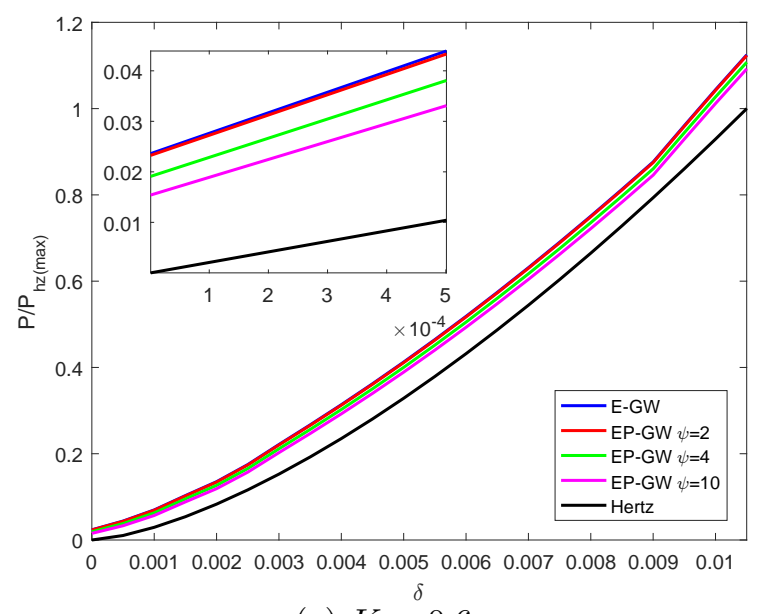

(a) $K=0.6$

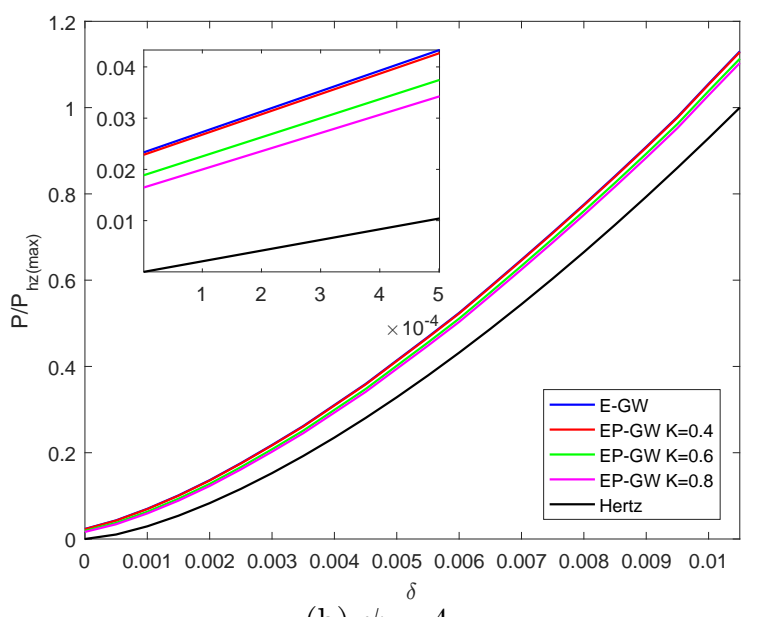

(b) $\psi=4$

Figure 4.24: Comparisons of the force-displacement relationship between different contact models

\subsection{The extended tangential GW model}

\subsubsection{Tangential contact force}

Frictional force is the resistive tangential force on the surfaces in contact, and for rough surfaces it develops on the areas of contacting asperities. Although the particle is assumed to be smooth in the DEM method, some tangential contact models are developed in DEM as introduced in Section 2.4.3 to calculate the tangential contact force between particles. To introduce the effects of surface roughness into the contact model in DEM, we must return to the basic understanding of the friction process.

Dry friction is a combined phenomenon of both mechanical deformation of surface irregularities and adhesive forces between two surfaces. The mechanical component of dry friction is governed by several factors such as normal load, surface mechanical properties, time of contact, temperature, surface roughness, and sliding velocity.

Amontons [186] made the most comprehensive contribution to modern ideas about frictionthe total friction force between two surfaces is directly proportional to the normal load and independent of the area of contact.

Back to the Coulomb's research on friction [187], the surface roughness is the major factor which contributes to the friction. Sometimes he showed the roughness as though they were like wire brushes, the bristles on one brush dragging their way through those on the other. Generally, it is suggested that if the average asperity angle is $\theta$, the coefficient of friction $\mu$ is equal to $\tan \theta$ and does not depend on the load or size of the bodies. The fact that the friction during sliding is often smaller than the static friction could be explained by assuming that the asperities on one surface could jump part of the way over the gap between asperities on the other [188], and the lubricants could reduce the friction by filling the hollows and reducing the effective roughness. The main weakness of the simple Coulomb model is that it 
is a non-dissipative process. The Coulomb model is illustrated in Figure 4.25, in which the action of rigid wedge-shaped asperities causes the two surfaces to move apart as they slide from position $\mathrm{A}$ to position $\mathrm{B}$. The coefficient of friction $\mu$ is equal to $\tan \theta$ by equating the work done by the frictional force to that done against the normal load. It is in considering the next phase of the motion. from $\mathrm{B}$ to $\mathrm{C}$, however, that the fundamental weakness of this model becomes apparent. Now the normal load does work on the system, and all the potential energy stored in the first phase of the motion (from A to B) is recovered. No net energy dissipation occurs in the complete cycle, thus no frictional force should be observable on a macroscopic scale if the interaction between real surfaces followed the Coulomb model exactly [189].

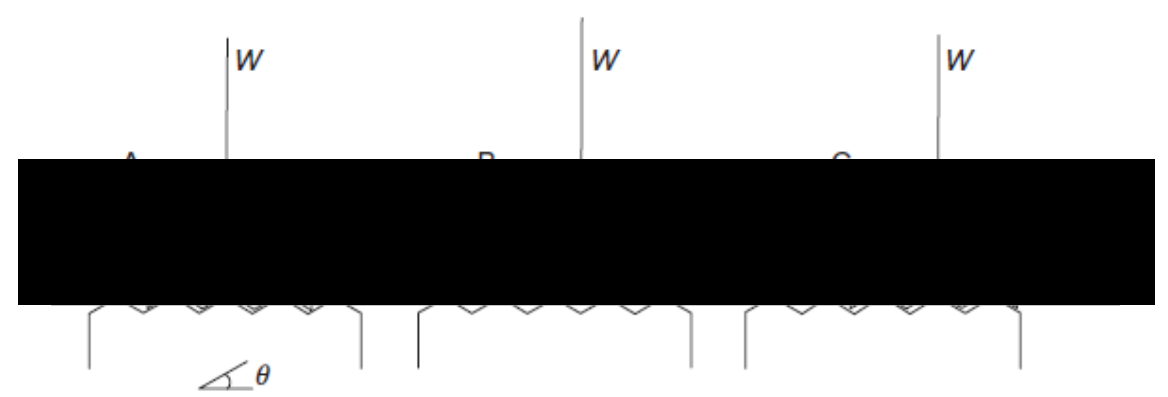

Figure 4.25: The schematic diagram of the Coulomb model [189]

Mindlin extended the Hertz theory by combining normal and tangential loadings to the bodies and considering slip exclusively at their contact interface [86]. In his theory, the contact interface consists of a central stick region surrounded by an annulus slip region. Mindlin and Deresiewicz proposed an incremental solution for the frictional elastic contact of two identical spheres that are subjected to varying normal and tangential forces. The MD theory for elastic frictional contact is limited to so-called simple loading histories, and includes eleven loading cases.

In more recent times, Tabor [190] experimentally verified and stated that the friction forces depended on three basic elements: (1) the area of true contact between the sliding surfaces; (2) the type of strength of bond that is formed at the interface where contact occurs; (3) the way in which the material in and around the contacting regions is sheared and ruptured during sliding.

Chang et al. [191] were the first to develop a static friction model for dry contact of rough surfaces in which Hamilton explicit formulae [192] are used to calculate the maximum tangential force that can be sustained before sliding inception. This tangential CEB model is not the same as the Mindlin approach because that the CEB model relates the start of full slip to material properties while the Mindlin model uses the local Coulomb friction law with a certain coefficient of friction. In this CEB model, the first yielding of a single material point is treated as a criterion for sliding inception. Kogut and Etsion [179] showed that this assumption underestimates the permissible tangential force because the first failed point is still surrounded by a considerable volume of elastic material which can support additional tangential loading. Roy Chowdhury and Ghosh [193] used slip and yield as two separate limiting criteria to obtain 
the total tangential force. They used Savkoor and Briggs [194] energy balance approach to attain the critical value of the tangential force at which slip occurs while for plasticity condition they used the von Mises criterion in conjunction with the Hamilton stress field. They assumed that with normally applied load some asperities get elastically deformed while others get plastically deformed and they ignored the intermediate elastic-plastic deformation of asperities. Again their model is for adhesive contact and as can be seen from most of the studies that the adhesion exists only on clean and very smooth surfaces and even in the adhesive contacts the contribution of adhesion effect in friction is not the domain one.

Waghmare and Sahoo [195] used the n-point asperity model framework [196] to study the friction at elastic-plastic contact of rough surfaces based on accurate finite element analysis of deformation of single asperity contact. In this model, the analysis of frictional force is carried out by following the energy balance approach of Johnson et al. [197] but with assumptions that (1) the surface energy or adhesion effect is negligible; and (2) plastically deformed asperities offer negligible resistance to motion. Thus friction force is offered only by the asperities that deform elastically and has two components. The first one is for the asperities which will slip before yielding under the combined effect of the normal load and the tangential force, and the second one for the asperities which will yield under the combined load. To obtain an expression for tangential force before slip, Savkoor and Briggs [194] followed the energy balance approach of JKR [197].

\subsubsection{Model description}

It can be seen that the mechanism of tangential force is very complicated and no widely accepted theory can be found now. In the current work, we try to tackle this problem based on the assumption in the GW model which leads to a straightforward cause of the resultant force in the tangential direction because of the asymmetric distribution of the normal pressure.

When a tangential displacement $\delta_{t}$ is defined between the sphere and the rough surface4.26, the asymmetric normal pressure will induce the tangential pressure along the contact area. $O$ is the initial origin point, $O^{\prime}$ is the new origin point with the tangential displacement $\delta_{t}$ and $C$ is the contact position between the sphere and the asperity. The distance from $C$ to $O$ is $r$ and the distance from $C$ to $O^{\prime}$ is $r_{1}$. The sphere is regarded as a rigid body. The contact force at point $C$ is defined as

$$
\begin{gathered}
p(r, \alpha)=\int_{\frac{r_{1}^{2}}{2 R}}^{+\infty}\left[z_{s}-\frac{r_{1}^{2}}{2 R}\right]^{3 / 2} \phi\left(z_{s}\right) d z_{s} \\
r_{1}=\sqrt{\left(r \cos \alpha-\delta_{t}\right)^{2}+(r \sin \alpha)^{2}}
\end{gathered}
$$




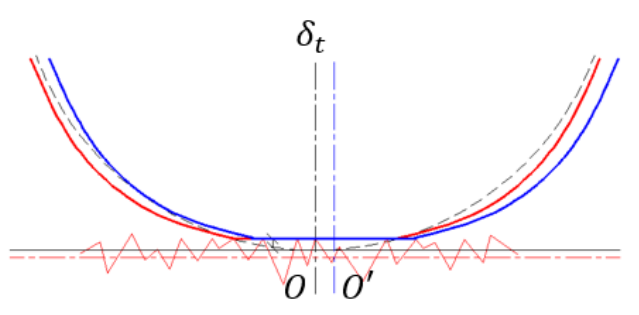

(a) Vertical view

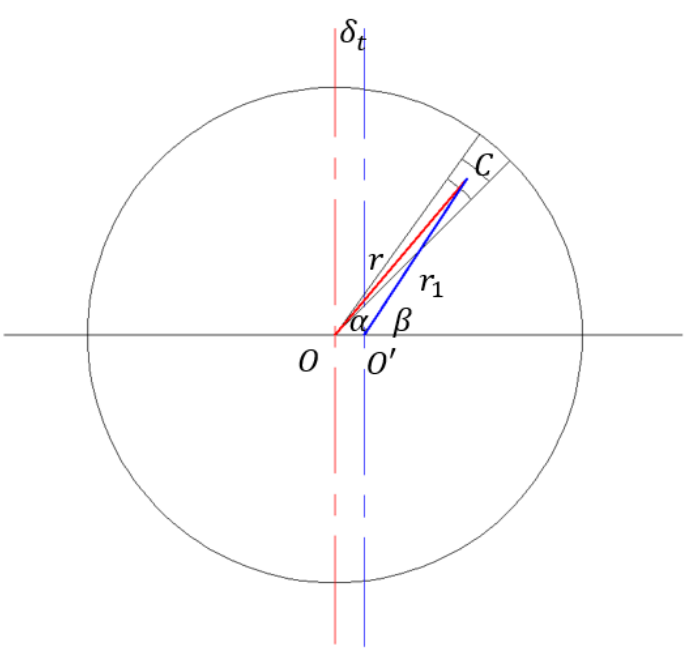

(b) Top view

Figure 4.26: Contact between sphere and rough surface with normal and tangential displacement

The above contact force can be decomposed into both normal and tangential directions

$$
\begin{aligned}
& p_{n}=p \frac{R-\delta}{\sqrt{(R-\delta)^{2}+r_{1}^{2}}} \\
& p_{t}=p \frac{r_{1}}{\sqrt{(R-\delta)^{2}+r_{1}^{2}}}
\end{aligned}
$$

The tangential force can then be decomposed into the $\mathrm{X}$ direction.

$$
\begin{gathered}
p_{t x}=p_{t} \cos \beta \\
\beta=\arccos \left(\frac{r \cos \alpha-\delta_{t}}{r_{1}}\right)
\end{gathered}
$$

The total normal force and tangential force can be obtained by integration

$$
\begin{gathered}
P_{n}=\int_{0}^{\pi} \int_{0}^{a} p_{n}(r, \alpha) r d r d \alpha \\
P_{t x}=\int_{0}^{\pi} \int_{0}^{a} p_{t x}(r, \alpha) r d r d \alpha
\end{gathered}
$$

The friction coefficient caused by the surface roughness is defined as

$$
\mu_{\text {rough }}=\frac{P_{t x}}{P_{n}}
$$

\subsubsection{Numerical results}

When $\delta=10^{-3}$ and $\sigma=10^{-3}$, the relation between $\mu$ and $\delta_{t}$ is shown in Figure 4.27. The normal and tangential pressure distributions when $\mu$ reaches the maximum are shown in 


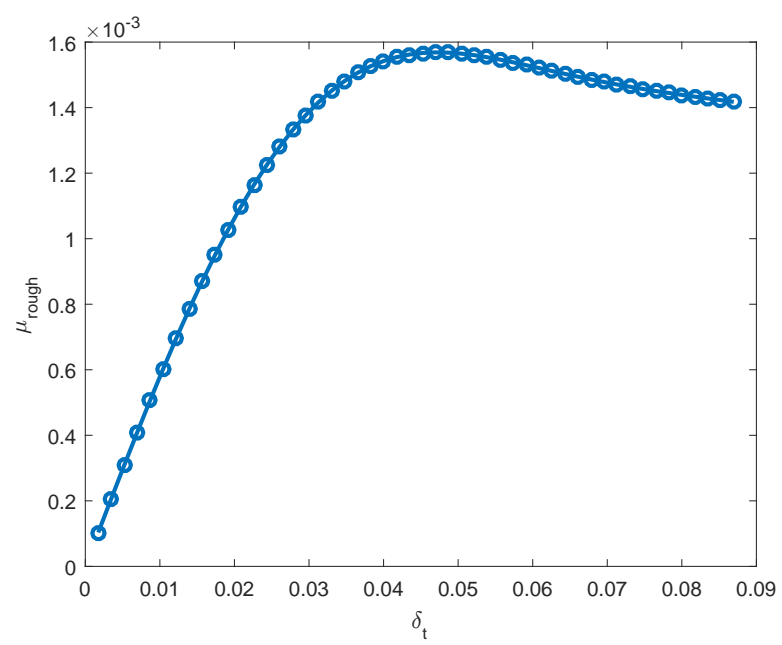

Figure 4.27: Coefficient of friction

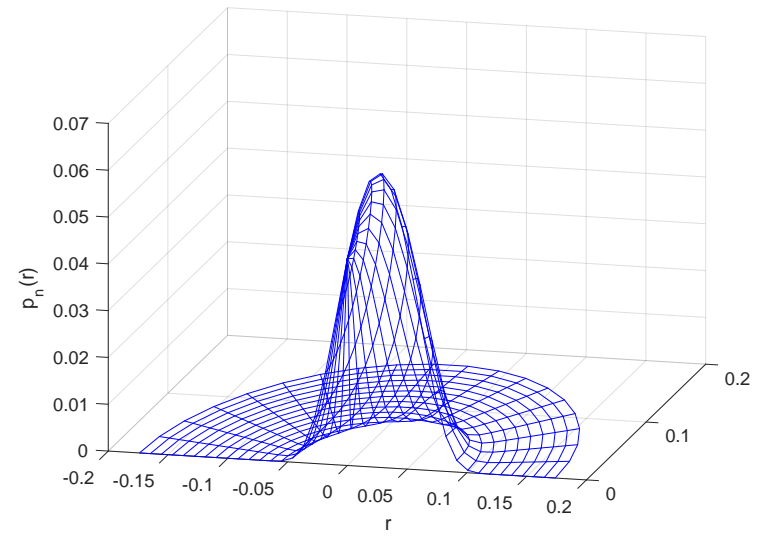

(a) Normal pressure distribution

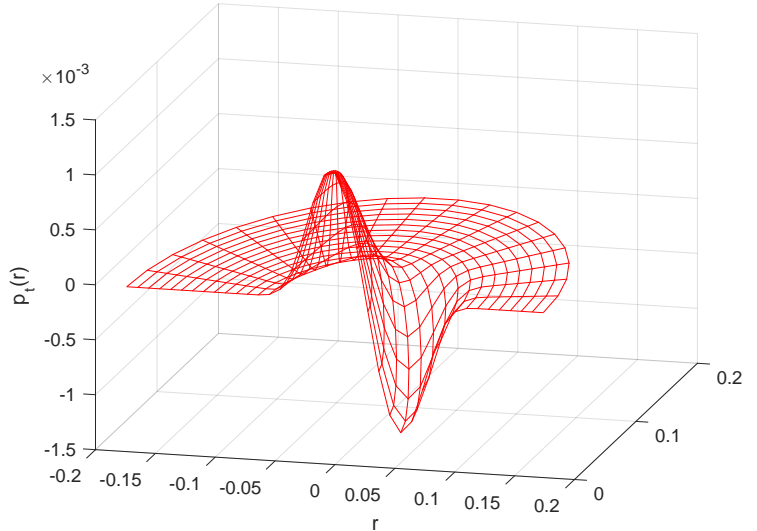

(b) Tangential pressure distribution

Figure 4.28: Pressure distribution

Figure 4.28. It can be seen that with the increase of displacement in the tangential direction, the phenomena of the asymmetric distribution of pressure is more and more obvious which leads to the corresponding increase of the coefficient of friction.

Figure 4.29 shows different $\delta_{t}-\mu$ curves with different $\sigma$ and $\mu$. When $\delta$ is fixed, $\sigma$ has a significant effect on the maximum of $\mu$ (three blue lines). Compared to $\sigma$, the effect of $\delta$ on $\mu$ is less obvious, especially when $\sigma$ is large (lines with same markers). The change of gradient of $\mu$ is not evident. When $\alpha<1$, slight change can be observed that when $\alpha$ increases the gradient of $\mu$ decreases. While when $\alpha>1$, the gradient of $\mu$ nearly stops changing. The relationship between $\alpha$ and gradient of $\mu$ is shown in Figure 4.30.

Figure 4.31(a) shows the relation between $\mu$ and $\sigma$ when $\delta$ is fixed. $\mu$ increases when $\sigma$ increases. Figure 4.31(b) shows the relation between $\mu$ and $\delta$ when $\sigma$ is fixed. $\mu$ keeps the same when $\delta$ increases.

One significant irrationality is that the magnitude of the coefficient of friction is very small. One possible explanation for it is that the tangential force caused by the asymmetric distribution of pressure distribution is only one factor that contributes to the total friction force. Although 


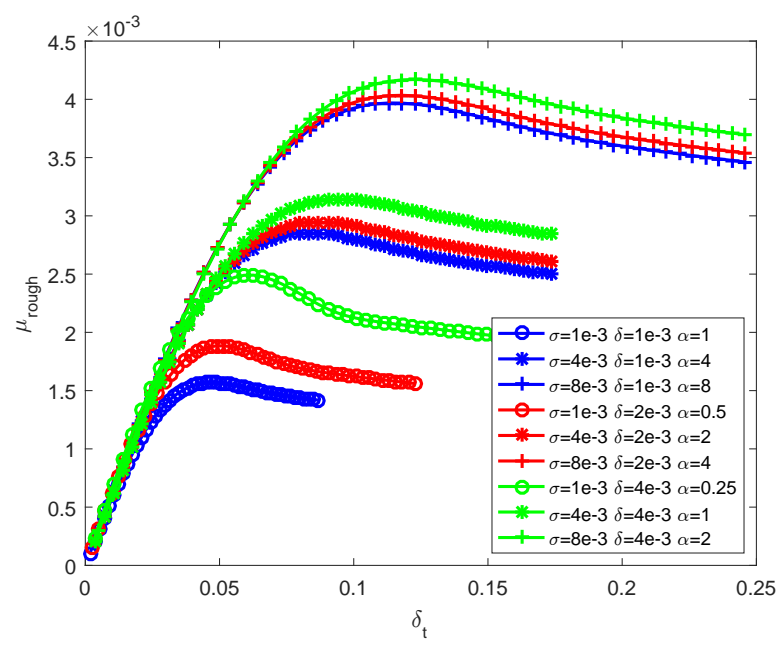

Figure 4.29: Coefficient of friction under different conditions

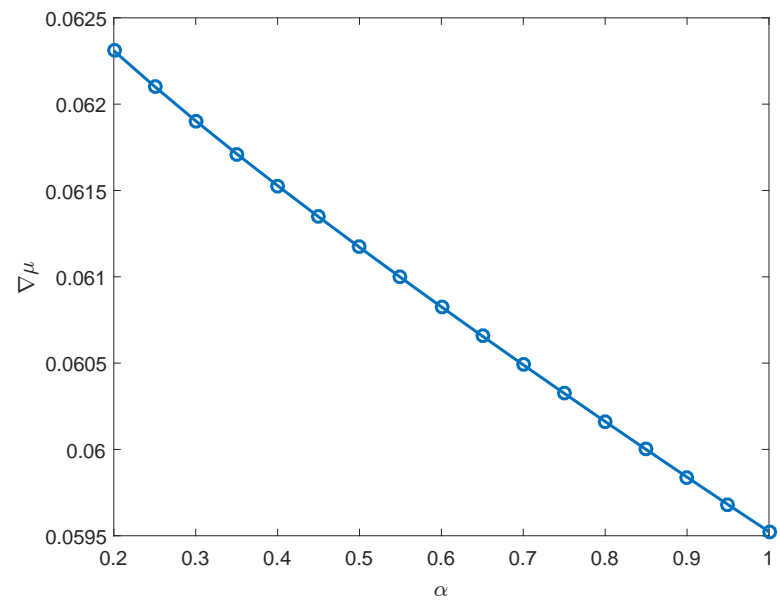

Figure 4.30: Variation of gradient of friction

it is not enough to analyse the friction force by only considering the influence of surface roughness by this mechanism, it is still worthy of being a useful complement to the existed theories of friction process. Therefore, the above simulations have been conducted to study the influences of the roughness parameters on the tangential force.

\subsection{The extended thermal conductivity GW model}

\subsubsection{Thermal resistance of rough surfaces}

Three types of heat transfer occur via a solid joint including radiative transfer, conduction through interstitial material in the gap, and conduction through the real contact area of which the thermal radiation can be neglected in most applications. As shown in Figure 4.32, heat transfer occurs through three main paths, the interstitial gas within the microgap $Q_{g}$, microcontacts $Q_{s}$, and the interstitial gas within the macrogap $Q_{G}$ [198]. As a result of the small real contact area and low thermal conductivities of interstitial gases, heat flow experiences a relatively large thermal resistance passing through the joint which leads to a 


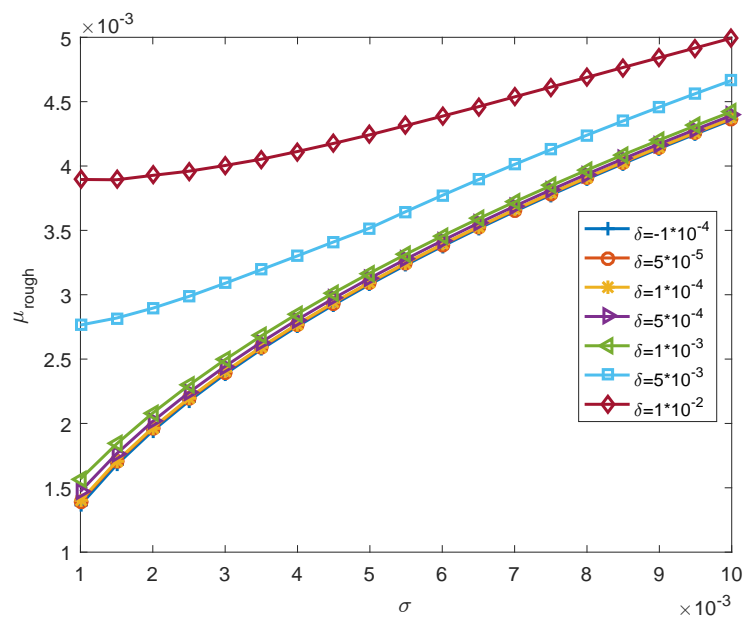

(a) Friction variation based on $\sigma$

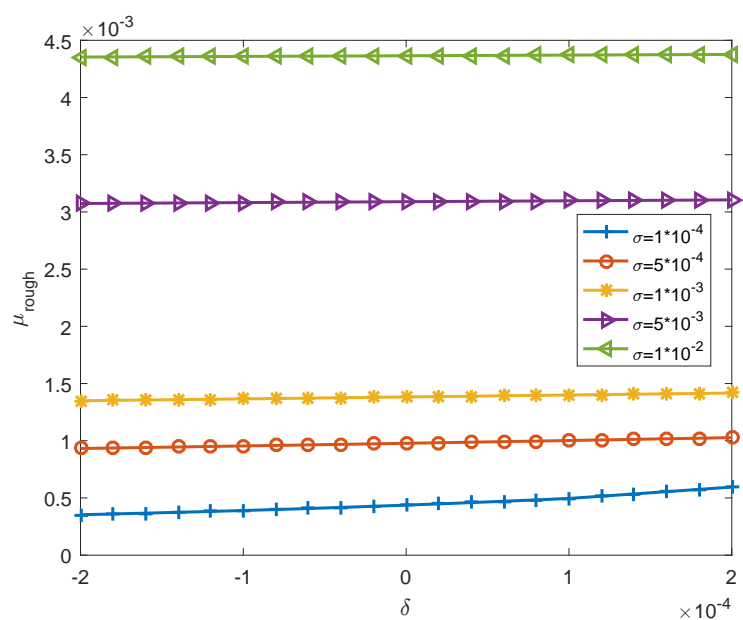

(b) Friction variation based on $\delta$

Figure 4.31: Variation of the coefficient of friction

relatively high-temperature drop across the interface.

Thermal constriction of the flux tube was employed as the basic theory for macro and micro thermal analysis. A simple accurate correlation is proposed for calculating the thermal spreading resistance of the isothermal flux tube [199].

$$
R=\frac{\psi(\varepsilon)}{2 k_{s} a}=\frac{(1-\varepsilon)^{1.5}}{2 k_{s} a}
$$

where $\psi(\cdot)$ is the spreading resistance factor, $k_{s}$ is the thermal conductivity, $a$ is the radius of the contact area, $b$ is the radius of the tube, and $\varepsilon=a / b$. When $a \ll b, R=1 / 2 k_{s} a$.

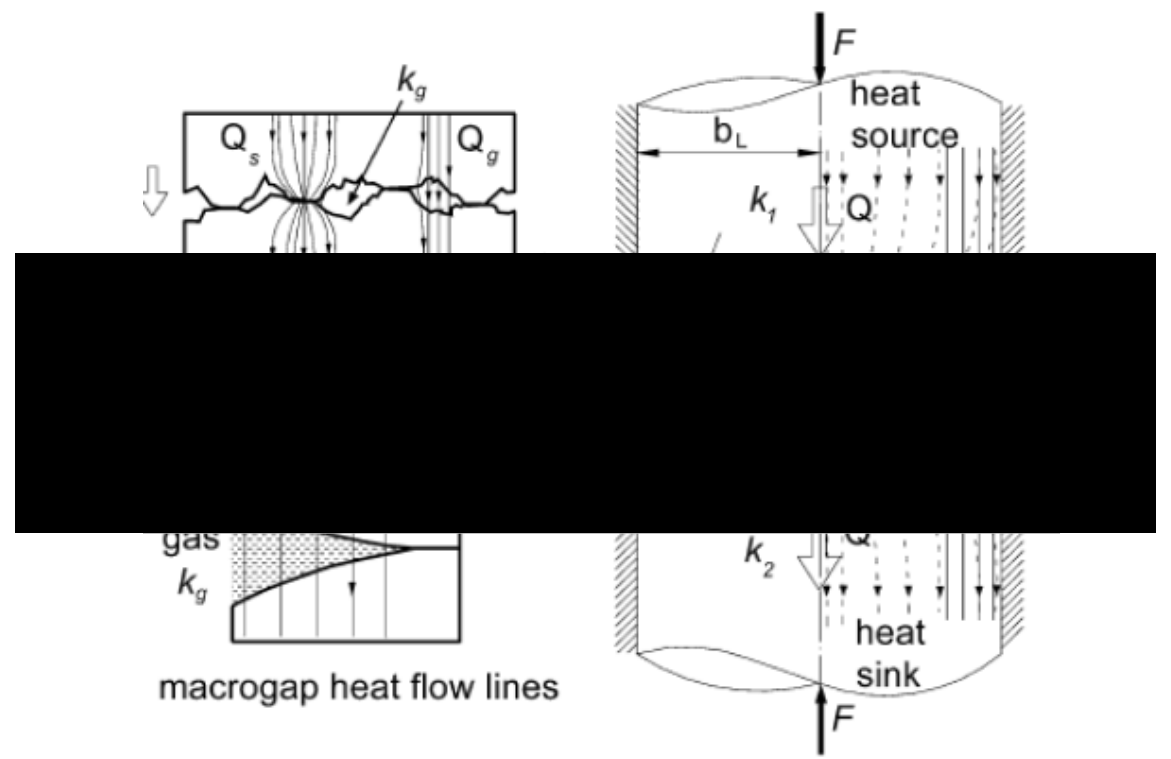

Figure 4.32: Conduction of nonconforming rough surfaces [198]

Several different thermal contact resistance models can be found in the literature. 
Thermal joint resistances model based on plastic asperities [198]

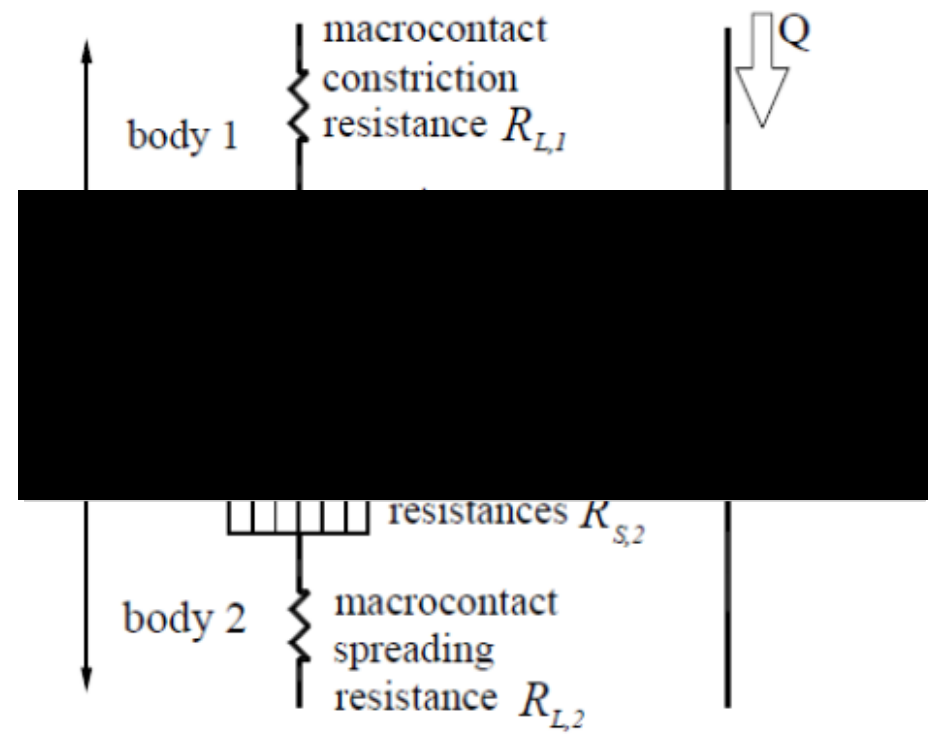

Figure 4.33: Thermal resistance network for nonconforming rough contacts in a vacuum [198]

Two sets of resistance in series can be used to represent the thermal contact resistance in a vacuum: the large-scale or macroscopic constriction resistance $R_{L}$, and the small-scale or microscopic constriction resistance $R_{S}$ as shown in Figure 4.33. Then the joint resistance $R_{j}$ can be expressed as

$$
\begin{gathered}
R_{j}=R_{\text {mic }}+R_{\text {mac }} \\
R_{j}=R_{L, 1}+R_{S, 1}+R_{S, 2}+R_{L, 2}
\end{gathered}
$$

where

$$
\left(\frac{1}{R_{S}}\right)_{1,2}=\left(\sum_{i=1}^{n_{S}} \frac{1}{R_{S, i}}\right)_{1,2}
$$

The flux tube solution is employed to determine the macrocontact and microcontact thermal resistances

$$
\begin{gathered}
R_{L}=\frac{\left(1-a_{L} / b_{L}\right)^{1.5}}{2 k_{s} a_{L}} \\
R_{S}(r)=\frac{\psi[\varepsilon(r)]}{2 k_{s} a_{s}(r)}
\end{gathered}
$$

In this model, asperities are assumed to deform plastically which means that the asperities are flattened during contact. That is to say that the asperities penetrate into the smooth surface in the equivalent model, without any change in shape of the parts of the equivalent rough surface not yet in contact. Therefore, bringing two rough surfaces together within a distance $Y$ is equivalent to removing the top of the asperities at a height $Y$ above the mean plane. The assumption of pure plastic microcontacts enables the micromechanics to be specified completely by the mean slope $m$ and the surface roughness $\sigma$, without having to assume some deterministic peak shapes. Cooper et al. [200] derived the following relationships for 
contact of nominal flat rough surfaces, assuming plastically deformed hemispherical asperities whose height and surface slopes have Gaussian distributions, where the mean separation $Y$ is constant throughout the contact plane

$$
\begin{gathered}
a_{s}=\sqrt{8 / \pi}(\sigma / m) \exp \left(\lambda^{2}\right) \operatorname{erfc}(\lambda) \\
n_{s}=\frac{1}{16}(m / \sigma)^{2}\left[\exp \left(-2 \lambda^{2}\right) / \operatorname{erfc} \lambda\right] A_{a} \\
A_{r} / A_{a}=\frac{1}{2} \operatorname{erfc} \lambda
\end{gathered}
$$

where $\lambda=Y / \sqrt{2 \sigma}, n_{s}, a_{s}, A_{r}$ and $A_{a}$ are respectively the dimensionless mean plane separation, number and average size of microcontacts, the real and the apparent contact area, and erfc $(\cdot)$ is the complementary error function.

YZ Li [201] correlated the surface slope $m$ and surface roughness $\sigma$ with a maximum error of $21 \%$.

$$
m=0.1399 \ln \sigma+0.1803
$$

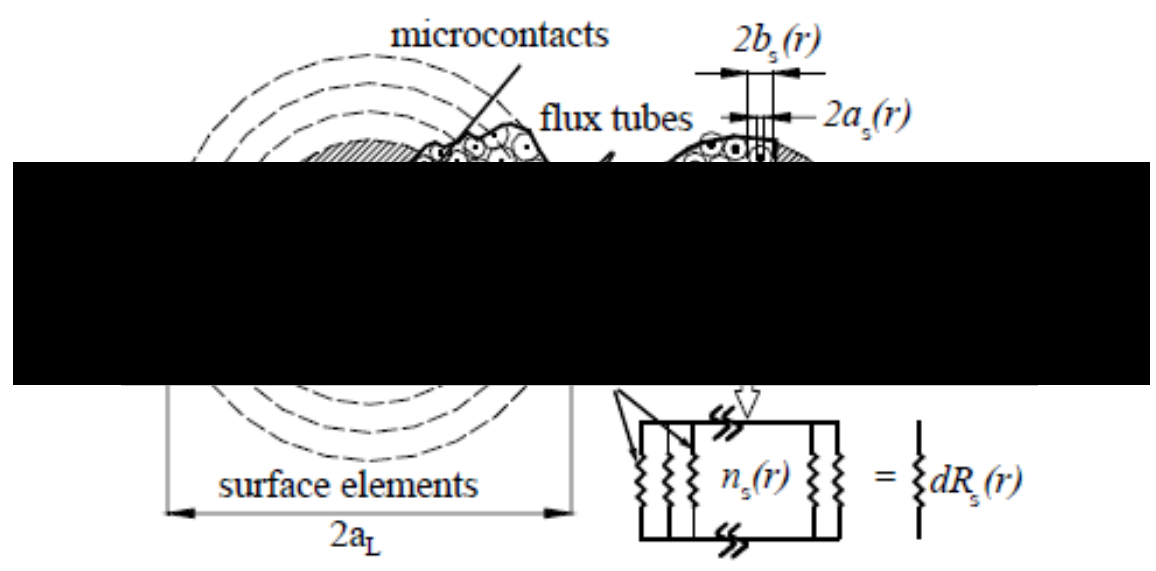

Figure 4.34: Microcontacts distribution in contact area and thermal resistance network for a surface element [198]

The thermal resistance network for the surface elements is shown in Figure 4.34. In each element $n_{s}(r)$ microcontacts exist which provide identical parallel paths for transferring thermal energy. Therefore, the microcontact thermal resistance for a surface element $d R_{s}(r)$ is

$$
d R_{s}(r)=\frac{R_{s}(r)}{n_{s}(r)}
$$

Surface elements form another set of parallel paths for transferring thermal energy in the macrocontact area. Therefore, the effective micro thermal resistance for the joint is

$$
R_{s}=\frac{1}{\sum 1 / d R_{s}(r)}
$$




\section{Thermal resistance based on the GW model}

Mikic [202, 203] derived expressions for the total thermal contact resistance (microscopic, $R_{c, S}$, plus macroscopic $R_{c, L}$ ) due to a nonuniform, axisymmetric contact pressure distribution

$$
\begin{gathered}
R_{c, S}=0.345 \frac{\sigma}{k m}\left[\int_{0}^{\infty} \frac{r}{b_{L}}\left(\frac{p}{H_{c}}\right)^{0.985} d\left(\frac{r}{b_{L}}\right)\right]^{-1} \\
R_{c, L}=8 \frac{b_{L}}{k} \sum_{\infty}^{n=1} \frac{\left[\int_{0}^{\infty} \frac{r}{b_{L}}\left(\frac{p}{p_{\text {avg }}}\right)^{0.985} J_{0}\left(\zeta_{n} \frac{r}{b_{L}}\right) d\left(\frac{r}{b_{L}}\right)\right]^{2}}{\zeta_{n} J_{0}^{2}\left(\zeta_{n}\right)}
\end{gathered}
$$

where $R_{c, S}$ is the resistance to heat flux caused by imperfect contact at the microscopic level due to roughness, $R_{c, L}$ is the resistance arising from macroscopic gaps between surfaces resulting from nonflatness, $k$ is the harmonic mean thermal conductivity, $H_{c}$ is the contact micro-hardness, $b_{L}$ is the radius of the contact surface, $J_{0}$ is the Bessel function, $\zeta_{n}$ is the $n^{\text {th }}$ root of the Bessel function, $p$ and $p_{\text {avg }}$ are the local and average contact pressures respectively. How to define the pressure distribution remains the crux of the problem. In this model, the pressure $p(r)$ is obtained by using the GW contact model.

\subsubsection{Model description}

From the above two models, we can see that when considering the thermal conductance (or resistance) of the contact, the contributions from the surface asperities and the bulk sphere are often evaluated respectively. This treatment is analogous to the basic idea in our extended GW model. Therefore, we attempt to apply the extended GW model in the thermal conduct problem. Equation (4.44) is used for calculating the conductance of every single asperity. Thus, the conductance of the asperity at the location $r$ is expressed as

$$
c_{G}(r)=2 k_{s} \beta^{1 / 2} \int_{d(r)}^{+\infty}\left[\delta_{G}(r)\right]^{1 / 2} \phi\left(z_{s}\right) d z_{s}
$$

The total conductance $C(\delta, \sigma)$ of the smooth sphere $C_{H}$ and the asperities $C_{G}$ can be expressed as

$$
C(\delta, \sigma)=\frac{C_{H}(\delta) C_{G}(\delta, \sigma)}{C_{H}(\delta)+C_{G}(\delta, \sigma)}
$$

where

$$
\begin{gathered}
C_{H}(\delta)=2 k_{s} \sqrt{R \delta} \\
C_{G}(\delta, \sigma)=\int_{0}^{\bar{a}} 2 \pi r c_{G}(r) d r
\end{gathered}
$$

The plastic contact model such as the ZMC model as described in Section 4.3.2 can also be applied to this thermal conductivity model to consider the plastic deformation of the asperities. 


\subsubsection{Numerical results}

A simple illustrative example is conducted to reflect this thermal conduct model based on the extended GW model. As shown in Figure 4.35, the influence of the asperities which will reduce the conductance of the smooth contact can be observed.

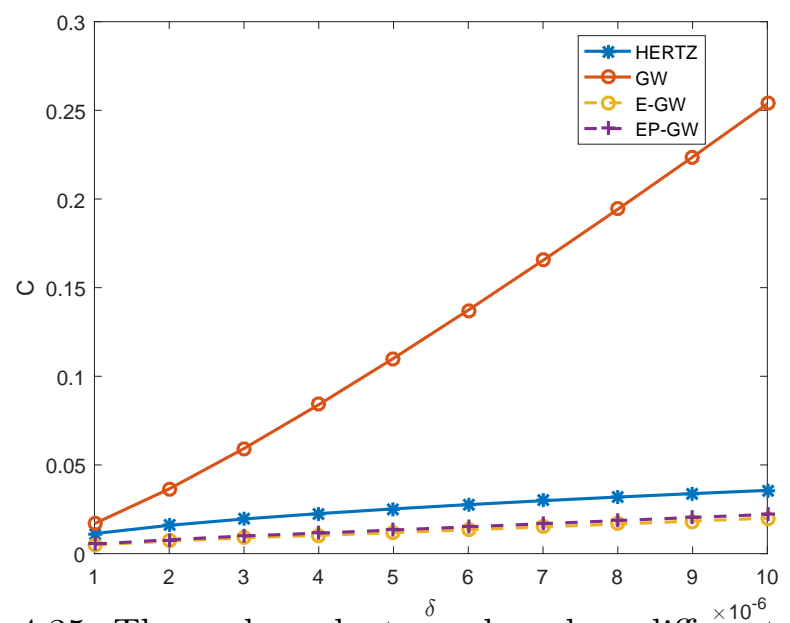

Figure 4.35: Thermal conductance based on different models

It is noted that this thermal model is only a preliminary result at the current stage. No comparison with other thermal resistance models is conducted as the parameters are not completely identical to other models.

\subsection{Concluding remarks}

Based on the theory and numerical solutions presented in Chapter 3, extensions of the classic GW model have been made from different perspectives in this chapter.

Firstly, as the classic GW model cannot handle the situation when the overlap between spheres is positive, an E-GW model is developed in Section 4.2 which considers the contribution of the asperities and the bulk sphere separately. Then the empirical normal contact law that can be used directly in DEM is derived by the curve-fitting method and incorporated in DEM code. Both one dimensional and three dimensional compression tests are conducted to illustrate the influence of the asperities of particles on the mechanical behaviour of the particulate system. Then, an EP-GW model is proposed in Section 4.3 in which the asperities can deform plastically using the ZMC elasto-plastic model. The numerical results show that the plastic deformation of the asperities reduces the pressure increased by the surface roughness.

Finally, the GW model is applied to consider influences of surface roughness on the tangential force and the thermal conductance in Section 4.4 and Section 4.5 respectively. However, this part of work is still preliminary. 


\section{Part III}

\section{Packing characterisation methods}




\section{Chapter 5}

\section{Characterising particle packings by PCA-2D}

\subsection{Introduction}

Particle packings play an important role in the discrete element method for modelling particulate systems as different packings can lead to different physical behaviour of the system, and therefore need to be properly characterised and controlled before being used for subsequent modelling. This is especially true for packings that are generated in a geometric fashion, see [204-210], for instance, for packing algorithms belonging to this category. Also in some critical applications, such as pebble bed reactors, detailed packing structures have an important impact on the system performance [211]. Therefore the spatial-statistical analysis of the geometrical structure of the system is of great scientific and engineering interests. As the topology of the system is highly complex, it is difficult to observe the way particles packed around each other by experiments. With the development of various particle based methods, such as Molecular Dynamics (MD) and the Discrete Element Method, more detailed information on the internal structure of particulate systems can be accessed.

Conventional means to characterise a particle packing are limited to: particle size distribution, packing density or porosity. A more computationally expensive method involves the use of a radial correlation function to exploit the spatial distribution of a packing, but it is less effective. It is also possible to check the coordination number distribution, and spatial isotropy via pairs of particle contacts, or their fabric tensor, when the packing is subject to (small) external loading. Nevertheless, in our opinion, there is still a lack of more general, comprehensive and quantitative approaches that can reveal some fundamental features of packings. In addition, several Monte Carlo samples of packing in DEM may need to be prepared for a given particle size distribution. However there are no existing approaches available that can compare and quantify the difference or similarity of such packings.

Currently, the techniques applied to investigate the features of particle packings focus on the 
packing density, orientations of the particle contacts, and internal (topological) structures of packings. Conventional means to characterise a particle packing are limited especially for loose packings without obvious contact chains. The classical characteristics such as particle size distribution and packing density cannot consider the spatial distribution of a packing. Besides, the method involves the use of a radial function is less effective [103]. When a packing is subject to external loading and generates contacts between particles, more features can be checked such as the coordination number, contact force distribution and the contact orientation distribution [212]. More advanced techniques have been developed to characterise a packing, see for instance [104].

These techniques may be classified into different categories from different perspectives. From the perspective of problem scale, there are macroscopic based (stress, strain and critical void ratio [105]) and microscopic based (coordination number and stress tensor [45]). While from the perspective of the specific information to be extracted from the particle system, the methods can be classified into two categories: One is focus on the void ratio (or the solid fraction) $[32,106]$ of the packing and the other pays attention on the contact network [107].

A particle packing can be viewed as a specific spatial variation of solid material (assigned a value of 1 ) and void (a value of 0 ). By discretisation of such a packing as a regular grid, and each grid cell is viewed as a pixel with a weighted value, the packing can be represented as a digitalised image with grey-scale ranging from 0 to 1 . Then the empirical covariance matrix of the image can be constructed and analysed by some techniques developed in the field of computer graphics and imaging processing [213, 214]. Furthermore, some measures [215, 216] are available that can be applied to compare the (dis)similarity of multiple images in a quantitative manner.

Principal component analysis (PCA) [217, 218], and its many variants under different names such as discrete Karhunen-Loeve or Hotelling transform is one of the most popular linear transform based statistical techniques, and has been extensively used in a wide variety of applications. It has proved to be a powerful tool that is often employed for data analysis in general, and dimension reduction in multi-variance analyses, and pattern recognition in signal and imaging processing in particular.

The current work attempts to develop a novel system that can characterise particle packings by using their principal components or variances obtained from PCA, and particularly it will prove that the principal variances can indeed qualify as the signature of a packing. Furthermore, a dissimilarity coefficient or a similarity index will be defined which provides a single valued metric to quantitatively compare two packings.

\subsection{Principal Component Analysis}

This section is devoted to the full description of the numerical procedure that is involved in principal component analysis of a packing and how such analysis can be applied to characterise 
particle packings. As only the main principles of PCA are adopted in the current work, some minor modifications are made and different terminologies are used when deemed to be more appropriate. As it is well known that PCA can be derived from Singular Value Decomposition [219], this is also applied for the current development.

\subsubsection{Packing digitalisation and formation of packing image}

First consider a circular particle assembly $\Omega_{p}=\bigcup_{i} \Omega_{i}$ where $\Omega_{i}$ is the domain of the $i$-th particle, and arbitrarily choose a rectangular region $\mathcal{A}$ of $L_{1} \times L_{2}$, termed the analysis window. The window can be divided into a regular grid of $M \times N$ square cells with spacing $h=L_{1} / M=L_{2} / N$. For a grid cell at $(i, j)$ with the area denoted as $\mathcal{A}_{i j}$, compute its average area ratio covered by particles, or grey-scale as

$$
a_{i j}=\frac{\left|\Omega_{p} \cap \mathcal{A}_{i j}\right|}{\left|\mathcal{A}_{i j}\right|}
$$

where $|\Omega|$ denotes the measure or area of a domain $\Omega ;|\mathcal{A}|=L_{1} L_{2}$; and $\left|\mathcal{A}_{i j}\right|=h^{2}$. An empty cell with no overlapping with any particle has $a_{i j}=0$; while a cell fully covered by a particle has $a_{i j}=1$. A cell partially covered by particles has $a_{i j}<1$. So in general $a_{i j} \in[0,1]$.

The collection of all the cell average area ratios $a_{i j}$ forms an $M \times N$ matrix $\mathbf{A}_{h}=\left\{a_{i j}\right\}$, which can be viewed as a digitalised grey-scale representation of the original packing $\Omega_{p}$ in the region $\mathcal{A}$, thus is termed as the packing matrix or image. Figure 5.1(b) illustrates such a digitalisation of a disc particle packing shown in Figure 5.1(a). Clearly, $\mathbf{A}_{h}$ is accurate within particles or void space of the packing, but may introduce approximation around particle boundaries. The accuracy of this representation for the original packing depends on the grid spacing $h$, and will be accurate in the limit case:

$$
\lim _{h \rightarrow 0} \mathbf{A}_{h}=\Omega_{p} \cap \mathcal{A}
$$

\subsubsection{Formulations and Numerical Procedures}

The mean value of the packing matrix $\mathbf{A}_{h}$, i.e. the packing density of the region $\mathcal{A}$, can be computed

$$
\rho_{\mathcal{A}} \equiv \frac{\left|\Omega_{p} \cap \mathcal{A}\right|}{|\mathcal{A}|}=\frac{1}{M N} \sum_{i=1}^{M} \sum_{j=1}^{N} a_{i j}
$$

Let $a(\mathbf{x})$ be the material distribution function with $a$ taking the value of 1 for a point within a particle, and 0 otherwise. It is not difficult to derive that the total variance of a packing in the region $\mathcal{A}$ is related to the packing density by

$$
\sigma_{\mathcal{A}}=\frac{1}{|\Omega|} \int_{\Omega}\left(a-\rho_{\mathcal{A}}\right)^{2} d \Omega=\rho_{\mathcal{A}}\left(1-\rho_{\mathcal{A}}\right)
$$




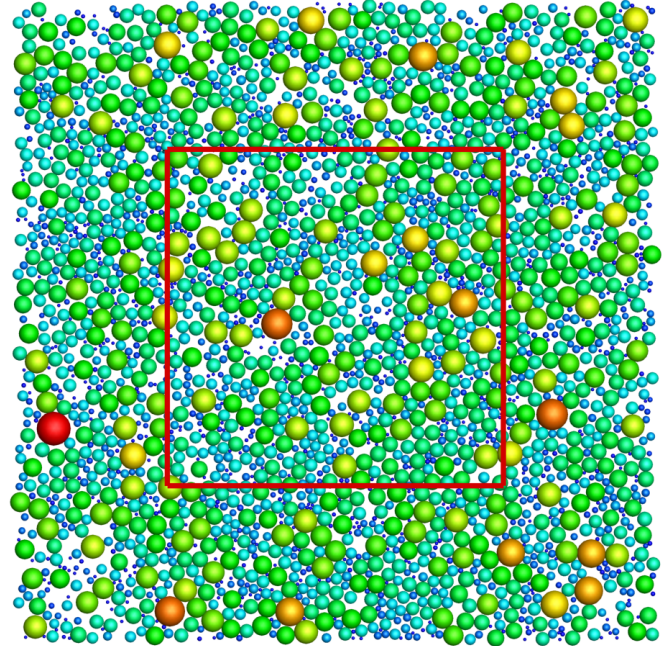

(a) a packing with a square analysis window

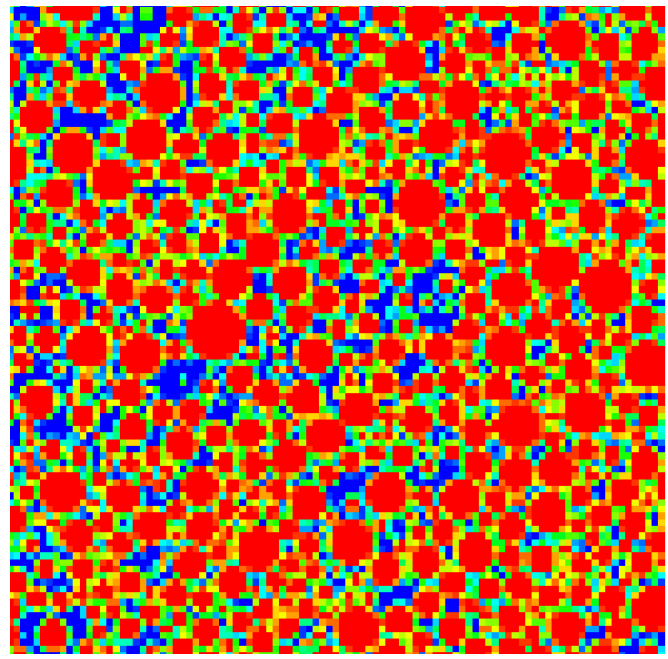

(b) a $100 \times 100$ digitalised image

Figure 5.1: A random particle packing and a digital representation within the analysis window

The total variance of the matrix is defined as

$$
\sigma_{h}=\frac{1}{M N} \sum_{i=1}^{M} \sum_{j=1}^{N}\left(a_{i j}-\rho_{\mathcal{A}}\right)^{2} \leq \sigma_{\mathcal{A}}
$$

i.e. the total variance of the packing $\sigma_{\mathcal{A}}$ is the upper bound of any packing image.

Let $m_{j}$ be the mean value of the $j$-th column of the packing matrix $\mathbf{A}_{h}$

$$
m_{j}=\frac{1}{M} \sum_{i=1}^{M} a_{i j}
$$

which also provides an alternative way to compute the density

$$
\rho_{\mathcal{A}}=\frac{1}{N} \sum_{j=1}^{N} m_{j}
$$

By subtraction of its mean from each column vector of $\mathbf{A}_{h}$, the column centralised matrix $\overline{\mathbf{A}}_{N}$ of $\mathbf{A}_{h}$ is obtained as:

$$
\overline{\mathbf{A}}_{N}=\mathbf{A}_{h}-\mathbf{e}_{M} \mathbf{m}_{N}
$$

where $\mathbf{e}_{M}$ is an $M \times 1$ column vector with all its elements being 1 's; and $\mathbf{m}_{N}$ is the $1 \times N$ mean value vector $\mathbf{m}_{N}=\left\{m_{j}\right\}$.

Define the covariance matrix of $\overline{\mathbf{A}}_{N}$ as

$$
\mathbf{S}_{N}=\frac{1}{M} \overline{\mathbf{A}}_{N}^{T} \overline{\mathbf{A}}_{N}
$$

where $\mathbf{S}_{N}$ is a $N \times N$ square matrix. Notice in the above that $M$ instead of $M-1$ is used. 
Further define the column-wise total variance as

$$
\sigma_{N}^{c}=\frac{1}{N} \operatorname{Tr}\left(\mathbf{S}_{N}\right)=\frac{1}{N} \sum_{i=1}^{N}\left(\mathbf{S}_{N}\right)_{i i}
$$

which may be (slightly) different from the total variance $\sigma_{h}$ in general.

By solving the eigenvalue problem of $\mathbf{S}_{N}$, it yields the following matrix decomposition

$$
\mathbf{S}_{N} \mathbf{V}_{N}=\mathbf{V}_{N} \mathbf{D}_{N}
$$

with

$$
\mathbf{D}_{N}=\mathbf{V}_{N}^{T} \mathbf{S}_{N} \mathbf{V}_{N}, \quad \mathbf{V}_{N}^{T} \mathbf{V}_{N}=\mathbf{I}_{N}
$$

where the diagonal matrix $\mathbf{D}_{N}=\operatorname{diag}\left\{d_{i}\right\}$ contains all the eigenvalues $d_{i}$ in descending order, which are termed the principal variances (PVs); and $\mathbf{V}_{N}=\left\{\mathbf{v}_{i}\right\}$ are the orthonormal vectors, termed the principal modes. As $\overline{\mathbf{A}}_{N}$ is column centralised, $\mathbf{S}_{N}$ is a semi-positive definite matrix with at least one zero principal variance. It is also well known that the sum of the $\mathrm{PVs}$ and the total column-wise variance is related by

$$
\frac{1}{N} \sum_{i=1}^{N} d_{i}=\sigma_{N}^{c}
$$

$\mathbf{S}_{N}$ can be recovered from the principal variances and modes as

$$
\mathbf{S}_{N}=\mathbf{V}_{N} \mathbf{D}_{N} \mathbf{V}_{N}^{T}=\sum_{i=1}^{N-1} d_{i} \mathbf{v}_{i} \mathbf{v}_{i}^{T}
$$

In many applications, only the first few principal variances are needed to approximate $\mathbf{S}_{N}$ to a reasonable degree, thereby significantly reducing the dimension of the problem concerned. This is often the main objective of PCA, but not an issue for the current problem.

Further define the projection $\mathbf{U}_{N}$ of $\overline{\mathbf{A}}_{N}$ onto the space spanned by $\mathbf{V}_{N}$ as

$$
\mathbf{U}_{N}=\overline{\mathbf{A}}_{N} \mathbf{V}_{N}
$$

Then it has

$$
\mathbf{D}_{N}=\frac{1}{N} \mathbf{U}_{N}^{T} \mathbf{U}_{N}
$$

and $\overline{\mathbf{A}}_{N}$ and $\mathbf{A}_{h}$ can be recovered by

$$
\overline{\mathbf{A}}_{N}=\mathbf{U}_{N} \mathbf{V}_{N}^{T} ; \quad \mathbf{A}_{h}=\overline{\mathbf{A}}_{N}+\mathbf{e}_{M} \mathbf{m}_{N}
$$

Similarly to $\mathbf{S}_{N}, \overline{\mathbf{A}}_{N}$ or $\mathbf{A}_{h}$ can be optimally approximated by the leading principal variances. The column-wise total variance $\sigma_{N}^{c}$, the mean value vector $\mathbf{m}_{N}$, the principal variance matrix $\mathbf{D}_{N}$ and the corresponding modes $\mathbf{V}_{N}$ form a unique set $\mathcal{S}_{\mathcal{N}}$, termed the column-wise 
characteristic set, that fully determines the packing in the vertical direction

$$
\mathcal{C}_{\mathcal{N}}=\left\{\sigma_{N}^{c}, \mathbf{m}_{N}, \mathbf{D}_{N}, \mathbf{V}_{N}\right\}
$$

As the PVs and the column-wise total variance is related by (5.12), and the total variance (and also the column-wise variance) is related to the density (see (5.4)), the PVs play a dominant role to characterise a packing image and therefore can be viewed as the (column-wise) signature of the packing.

The above are developed based on the column-wise consideration of the matrix $\mathbf{A}_{h}$. Equally, another set can be derived following a row-wise consideration. By labelling the mean value (column) vector for the rows of $\mathbf{A}_{h}$ as $\mathbf{m}_{M}$, and the row-centralised covariance matrix $\overline{\mathbf{A}}_{M}$ as

$$
\bar{A}_{M}=\mathbf{A}_{h}-\mathbf{m}_{M} \mathbf{e}_{N}^{T}
$$

where $\mathbf{e}_{N}$ is a $N \times 1$ vector of all 1's, and the corresponding covariance matrix of $M \times M$ as

$$
\mathbf{S}_{M}=\frac{1}{N} \bar{A}_{M} \bar{A}_{M}^{T}
$$

the row-wise total variance can be computed as

$$
\sigma_{M}^{r}=\frac{1}{M} \operatorname{Tr}\left(\mathbf{S}_{M}\right)=\frac{1}{M} \sum_{i=1}^{M}\left(\mathbf{S}_{M}\right)_{i i}
$$

The $M$ principal variances and modes can be found as

$$
\mathbf{S}_{M} \mathbf{V}_{M}=\mathbf{V}_{M} \mathbf{D}_{M}, \quad \mathbf{V}_{M}^{T} \mathbf{V}_{M}=\mathbf{I}_{M}
$$

Consequently, the row-wise characteristic set of $\mathcal{A}$ becomes

$$
\mathcal{C}_{\mathcal{M}}=\left\{\sigma_{M}^{r}, \mathbf{m}_{M}, \mathbf{D}_{M}, \mathbf{V}_{M}\right\}
$$

and $\mathbf{D}_{M}$ are the (row-wise) signature of the packing image. As $\mathbf{m}_{N}$ and $\mathbf{m}_{M}$ are generally different even when $N=M$, so $\mathbf{S}_{N} \neq \mathbf{S}_{M}$, and thus $D_{N} \neq D_{M}$. The difference will depend on $N$ and $M$, and the packing configuration.

For simplicity, only the column-wise related PCA is discussed below.

\subsubsection{Principal Variance Function}

To facilitate the comparison between different sets of principal variances, particularly when they are obtained from different resolutions $N$, the ordinal number $i$ of a principal variance $d_{i}$ is mapped from 1 to $N$ to a non-dimensional "position" variable $x \in[0,1]$ by

$$
x(i)=\frac{1}{N}\left(i-\frac{1}{2}\right)
$$


Then a continuous function $\mathrm{d}(x), x \in[0,1]$, termed the (column-wise) principal variance function, can be constructed to interpolate the discrete variances $d_{i}$ using piecewise linear or higher order interpolation functions such that

$$
\mathrm{d}\left(x_{i}\right)=d_{i}, \quad x_{i}=x(i), \quad i=1, \cdots, N
$$

The conversion from the ordinal number $i$ to the position $x$ play an essential role to quantitatively compare two images, with different or same resolutions.

\subsubsection{Packing Image Similarity}

Consider two packing images with their principal variance functions $\mathrm{d}_{1}(x)$ and $\mathrm{d}_{2}(x)$ obtained, define a so-called dissimilarity coefficient (DC) between these two images as

$$
\mathcal{D}_{c}=\left[\frac{1}{\Sigma_{1}+\Sigma_{2}} \int_{0}^{1}\left[\mathrm{~d}_{1}(x)-\mathrm{d}_{2}(x)\right]^{2} d x\right]^{1 / 2} \in[0,1]
$$

where $N_{1}$ and $N_{2}$ are respectively the (column) resolutions of the two images; and $\Sigma_{1}$ and $\Sigma_{2}$ are defined as

$$
\Sigma_{i}=\int_{0}^{1} \mathrm{~d}_{i}^{2}(x) d x \quad(i=1,2)
$$

Consequently, the degree of similarity of these two packing images can be quantified by the similarity index $\in[0,100]$ defined as

$$
\mathcal{S}_{\mathcal{I}}=\left(1-\mathcal{D}_{c}\right) \times 100
$$

\subsection{Principal Variances and Modes of Some Special Packings}

This section exploits what additional properties of the principal variances and modes that some special packing configurations/images may have. Illustrative examples are provided to validate the theoretical development in this section.

\subsubsection{Permuted, Mirrored Packings}

For a given packing matrix $\mathbf{A}_{N}$, assume that another matrix $\mathbf{A}_{N}^{\prime}$ is obtained by permutating columns. Let the permutation be represented by a permutation matrix $\mathbf{P}_{N}$ of order $N \times N$. As $\mathbf{P}_{N}^{T} \mathbf{P}_{N}=\mathbf{I}_{N}$ it is not difficult to prove that $\mathbf{A}_{N}^{\prime}$ will have the same principal variances but the principal modes are the permutation of the original ones

$$
\mathbf{D}_{N}^{\prime}=\mathbf{D}_{N} ; \quad \mathbf{V}_{N}^{\prime}=\mathbf{P}_{N} \mathbf{V}_{N}
$$

A similar conclusion can be drawn if a matrix is obtained from row permutation. 
Notice that permutation can mirror or reflect a packing and therefore it can be concluded that the mirror packing has the same principal variances as the original packing.

\subsubsection{Repetitive, Periodic and Symmetric Packings}

If the packing has a repetitive or periodic structure where a basic packing unit repeats multiple times along the horizontal direction, PCA can be applied to this basic packing and the principal variances and modes of the whole structure can be readily obtained, as derived below.

Consider the simplest 2-repetition case where the basic structure is repeated twice. Use the same grid spacing for both the basic structure and the whole packing, and let $\mathbf{A}_{N}$ be the packing matrix of the basic packing. Then the whole packing matrix, $\mathbf{A}_{2 N}$, consists of two identical sub-matrices $\mathbf{A}_{N}$

$$
\mathbf{A}_{2 N}=\left[\mathbf{A}_{N}, \mathbf{A}_{N}\right]
$$

Let $\mathbf{m}_{N}, \overline{\mathbf{A}}_{N}$ and $\mathbf{S}_{N}$ be respectively the column mean vector, the mean centralised matrix and the covariance matrix of $\mathbf{m}_{N}$ as defined before. Then for $\mathbf{A}_{2 N}$, the column mean vector is

$$
\mathbf{m}_{2 N}=\left\{\mathbf{m}_{N}, \mathbf{m}_{N}\right\}
$$

which leads to the mean centralised matrix

$$
\overline{\mathbf{A}}_{2 N}=\mathbf{A}_{2 N}-\mathbf{e}_{M} \mathbf{m}_{2 N}
$$

The covariance matrix takes the form

$$
\mathbf{S}_{2 N}=\frac{1}{M} \overline{\mathbf{A}}_{2 N}^{T} \overline{\mathbf{A}}_{2 N}=\frac{1}{M}\left[\begin{array}{ll}
\mathbf{S}_{N} & \mathbf{S}_{N} \\
\mathbf{S}_{N} & \mathbf{S}_{N}
\end{array}\right]
$$

Again let $\mathbf{V}_{N}$ and $\mathbf{D}_{N}$ be respectively the principal variances and modes of $\mathbf{S}_{N}$. Now construct a matrix of order $2 N \times N$

$$
\mathbf{V}_{2 N}=\frac{1}{\sqrt{2}}\left[\begin{array}{l}
\mathbf{V}_{N} \\
\mathbf{V}_{N}
\end{array}\right]
$$

Then

$$
\mathbf{S}_{2 N} \mathbf{V}_{2 N}=\frac{1}{\sqrt{2}}\left[\begin{array}{l}
2 \mathbf{S}_{N} \mathbf{V}_{N} \\
2 \mathbf{S}_{N} \mathbf{V}_{N}
\end{array}\right]=\frac{1}{\sqrt{2}}\left[\begin{array}{l}
2 \mathbf{V}_{N} \mathbf{D}_{N} \\
2 \mathbf{V}_{N} \mathbf{D}_{N}
\end{array}\right]=\mathbf{V}_{2 N}\left(2 \mathbf{D}_{N}\right)
$$

i.e. $\mathbf{V}_{2 N}$ are the principal modes of $\mathbf{S}_{2 N}$ and the corresponding principal variances are 2 times $\mathbf{D}_{N}$. Note, however, that $\left(\mathbf{V}_{2 N}, 2 \mathbf{D}_{N}\right)$ only account for $N$ or half principal pairs of $\mathbf{S}_{2 N}$.

Construct another matrix of order $2 N \times N$

$$
\mathbf{V}_{2 N}^{\prime}=\frac{1}{\sqrt{2}}\left[\begin{array}{c}
\mathbf{V}_{N} \\
-\mathbf{V}_{N}
\end{array}\right]
$$


Table 5.1: Total variances of three regular packings with different grid resolutions

\begin{tabular}{|c|c|c|c|c|}
\hline \multicolumn{2}{|c|}{ Grid } & \multicolumn{3}{c|}{ Packing } \\
\hline $\mathrm{N}$ & $\mathrm{h}$ & $\mathrm{R} 1$ & $\mathrm{R} 4$ & $\mathrm{R} 16$ \\
\hline 25 & 0.04 & $\mathbf{. 1 5 0 1}$ & .1362 & .1112 \\
\hline 50 & 0.02 & .1589 & $\mathbf{. 1 5 0 1}$ & .1362 \\
\hline 100 & 0.01 & .1636 & .1589 & $\mathbf{. 1 5 0 1}$ \\
\hline
\end{tabular}

Direct calculation leads to

$$
\mathbf{S}_{2 N} \mathbf{V}_{2 N}^{\prime}=0
$$

which means that $\mathbf{V}_{2 N}^{\prime}$ are also the principal modes, but the corresponding principal variances are all zero. Combination of (5.33) and (5.35) concludes that the half of the principal variances of the whole packing are 2 times those of the basic structure, but the rest are zero.

In general, for a $m$-repetition packing, $1 / m$ of the principal variances will be $m$ times those of the basic structure, and the remaining principal variances are zero.

If the repetition occurs in the vertical direction, the whole packing matrix admits the following partition for a 2-repetition packing

$$
\mathbf{A}_{2 N}=\left[\begin{array}{l}
\mathbf{A}_{N} \\
\mathbf{A}_{N}
\end{array}\right]
$$

Utilising the fact that $\mathbf{m}_{2 N}=\mathbf{m}_{N}$, one can prove that

$$
\mathbf{S}_{2 N}=\mathbf{S}_{N}
$$

hence the principal variances and modes remain the same. This repetitive feature will be exploited, nevertheless, when applying the row-wise PCA to the packing matrix.

If a packing is symmetric about a vertical line, one part can be obtained by mirroring the other part against the symmetric line through column permutation, and so both parts will have the same principal variances. Thus for the whole packing, the non-zero principal variances will be twice those from each part.

It is also obvious that an exact scaling of both the packing and the image grid together will lead to the principal variances and modes being unchanged.

\subsubsection{Numerical Validation}

Consider three regular packings within a unit square region as show in Figure 5.2(a-c), where there are respectively 1, 4 and 16 equal-sized particles in the packings that are labelled as R1, R4 and R16 respectively. The three packings have nested or exactly scaled configurations, and have the same packing density $\rho=\pi / 4=0.7854$. The region is then divided into a $N \times N$ square grid with spacing $h=1 / N$ for each packing, and three resolutions $N=25,50$ and 100 


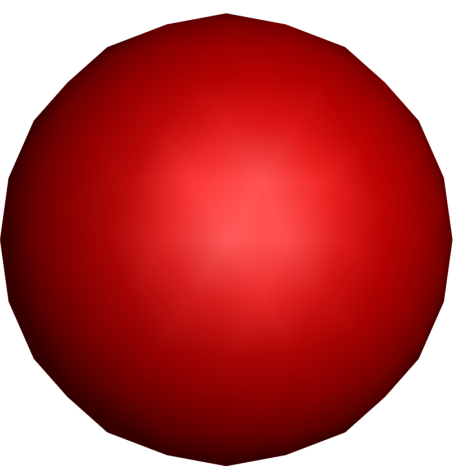

(a) Packing R1

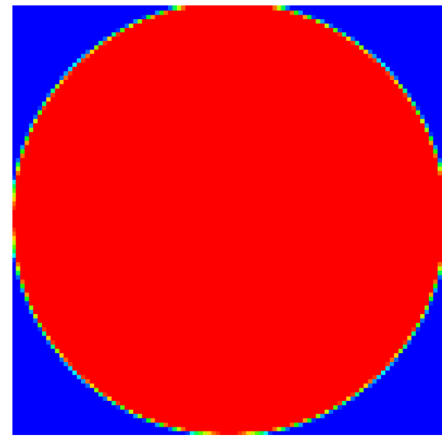

(d) Image $\mathrm{R} 1(\mathrm{~N})$

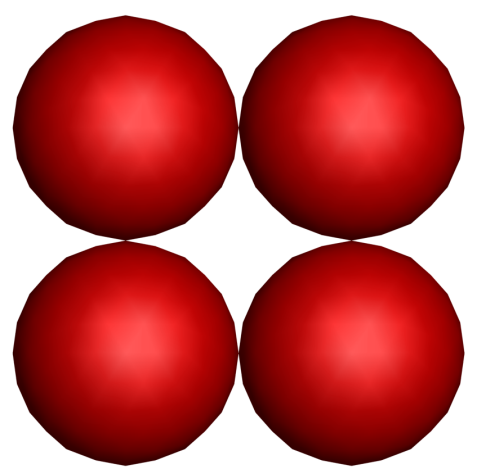

(b) Packing R4

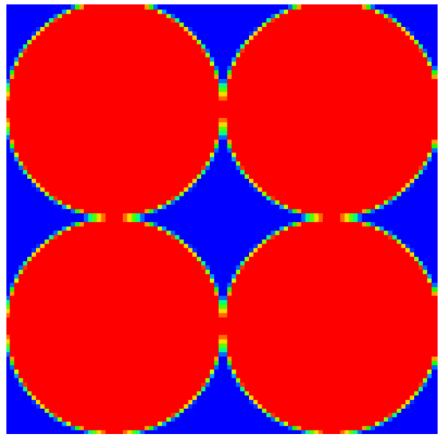

(e) Image $\mathrm{R} 4(\mathrm{~N})$

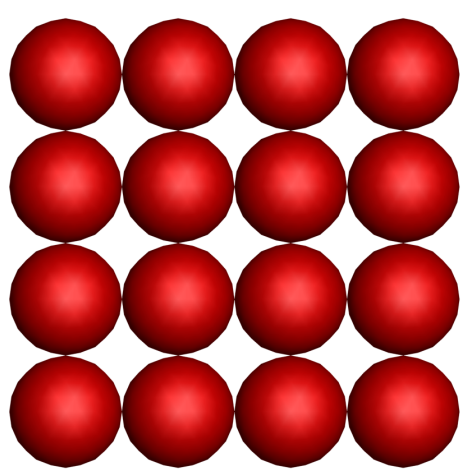

(c) Packing R16

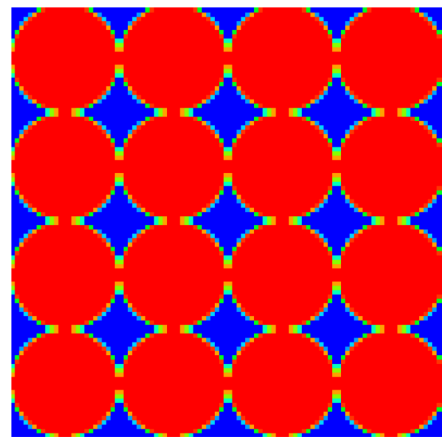

(f) Image R16(N)

Figure 5.2: Three regular packings (a-c) and their digitalised images (d-f) $(\mathrm{N}=100)$

Table 5.2: The first principal variances of three regular packings with different grid resolutions

\begin{tabular}{|c|c|c|c|c|}
\hline \multicolumn{2}{|c|}{ Grid } & \multicolumn{3}{c|}{ Packing } \\
\hline $\mathrm{N}$ & $\mathrm{h}$ & $\mathrm{R} 1$ & $\mathrm{R} 4$ & $\mathrm{R} 16$ \\
\hline 25 & 0.04 & $\mathbf{1 . 7 6 6 5}$ & 1.7050 & 1.5133 \\
\hline 50 & 0.02 & 3.5778 & $\mathbf{3 . 5 3 2 9}$ & 3.4099 \\
\hline 100 & 0.01 & 7.1816 & 7.1556 & $\mathbf{7 . 0 6 5 7}$ \\
\hline
\end{tabular}

are considered. The resulting packing images are labelled as $\operatorname{Ri}(N)(i=1,4,16 ; N=1,50,100)$. The images with $N=100$ are depicted in Figure 5.2(d-f). The total column-wise variances and first or maximum principal variances of the three packings for different grid resolutions are respectively given in Tables 5.1 and 5.2.

Table 5.1 shows that the total column-wise variance exhibits a clear pattern: the values along the main diagonal and two off-diagonal lines are the same. This can be easily explained due to the nested relationship between the three packings.

For the first principal variances listed in Table 5.2, a pattern also emerges along the main diagonal and two off-diagonal lines: the ratio between two consecutive values on a line is exactly 2 . This validates the theoretical derivation in this section for repetitive packings. For instance, Image R4(50) is a 2-repetition of Image R1(25) (with an additional 2-repetition in the vertical direction but having no contribution to the PVs), leading to doubled PVs for the 


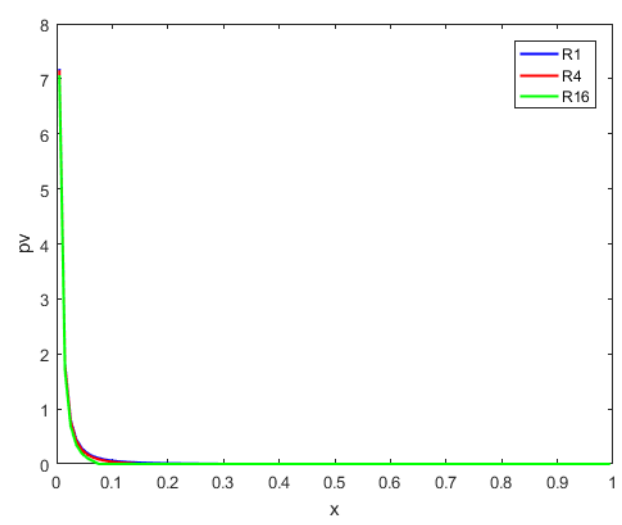

(a) Normal view

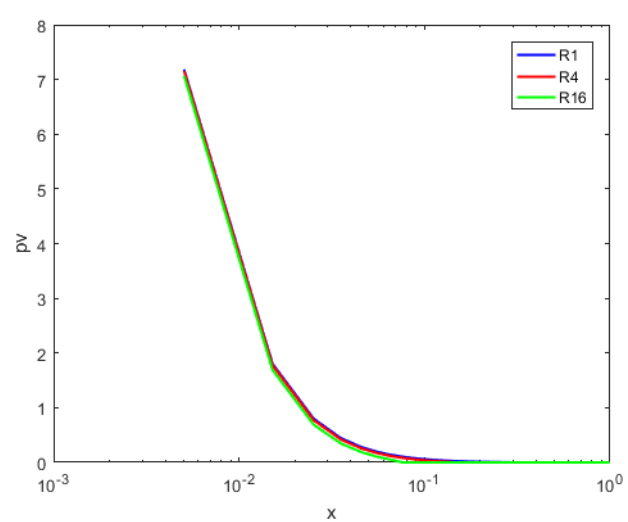

(b) Zoomed view

Figure 5.3: Principal variances of $\mathrm{R} 1, \mathrm{R} 4$ and $\mathrm{R} 16$ at $\mathrm{N}=100$

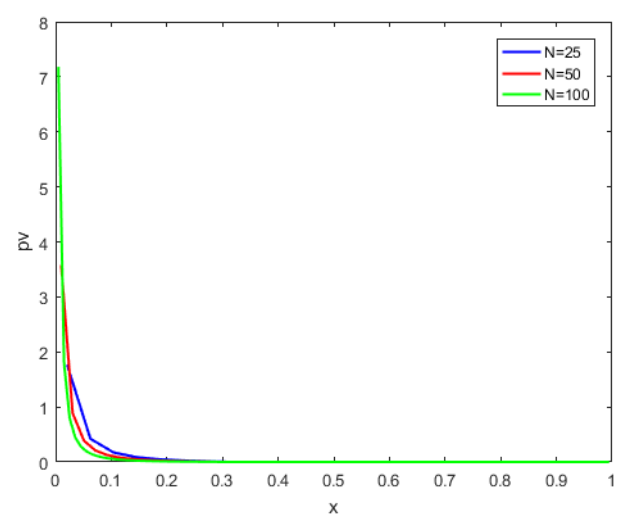

(a) Normal view

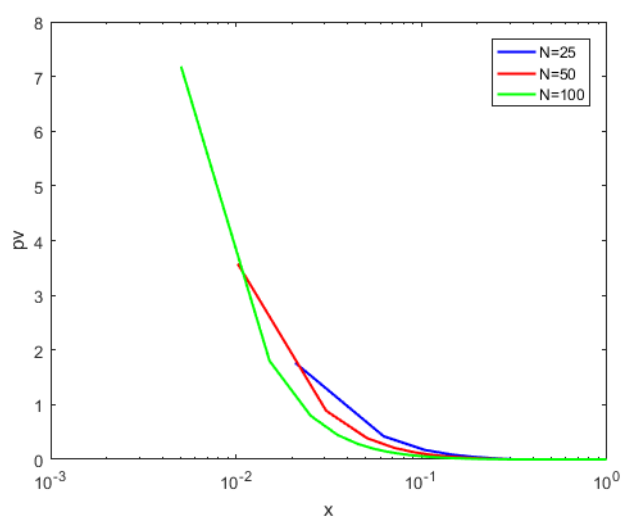

(b) Zoomed view

Figure 5.4: Principal variances of $\mathrm{R} 1$ at $\mathrm{N}=25,50,100$

former; While Image R16(100) is also a 2-repetition packing of Image R4(50), thus having PVs which are double of those of R4.

It is also observed, but without offering a rigorous proof, that for a quarter of disc which has a diagonal symmetry and is the basic building block of a disc, the number of non-zero principal variances is equal to $\lfloor N(2-\sqrt{2}) / 2\rfloor$, where $\lfloor\cdot\rfloor$ is the floor function.

The PVs of the three packings with $N=100$ are plotted in Figure 5.3(a), and also in Figure 5.3(b) with a logarithmic scale of $x$ to achieve a zoomed view for the leading PVs. Both Table 5.2 and Figure 5.3 show that very similar principal variances are obtained from the three packings with the same grid resolution $N$. In fact, the dissimilarity coefficients of R4 and R16 against R1 are 0.0081 and 0.0275 respectively. The increased dissimilarity for $\mathrm{R} 16$ is due to the reduced relative resolution in terms of ratio $r / h$.

Figure 5.4(a) displays all the PVs of Packing R1 for the three grid resolutions, with a zoomed view for the main PVs shown in Figure 5.4(b). Both Table 5.2 and Figure 5.4 demonstrate the dependence of the VPs on the grid resolution $N$. This issue will be further investigated in 


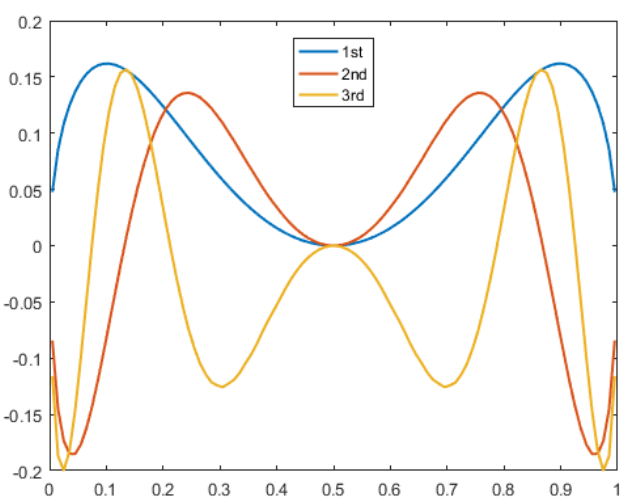

(a) First three modes of R1(100)

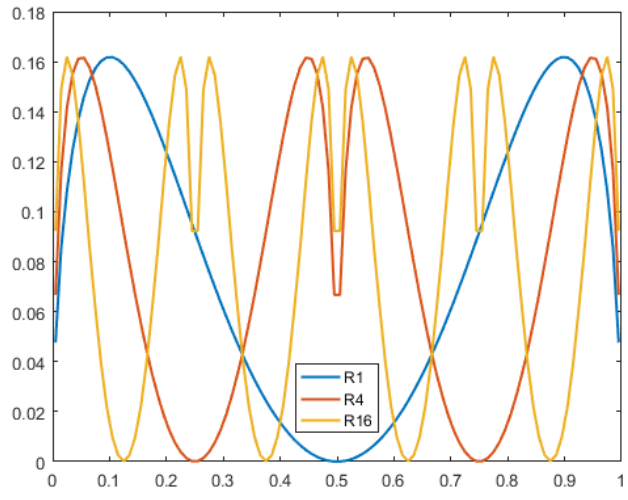

(b) First models of R1(100), R4(100) and $\mathrm{R} 16(100)$

Figure 5.5: Some leading principal modes of R1, R4 and R16 at N=100

Section 5.4.5.

The first three principal modes of R1(100), and the first modes of R1, R4 and R16 with $\mathrm{N}=100$ are plotted in Figure 5.5(a) and (b) respectively. The symmetric nature of all three modes of R1(100) is evident as expected, while the first modes of R4(100) and R16(100) are compressed and repetitive versions of the base case $\mathrm{R} 1(100)$.

\subsection{Packing Characterisation using Principal Variances}

As the main signature of a packing, principal variances will be comprehensively exploited in this section to understand how they can be applied to characterise a packing or to quantitatively compare the similarity or difference between different packings. In addition to the three regular packings that have been used to validate some of our theoretical developments for repetitive packings, additional two sets of random packings will be utilised to provide new evidence as the basis for further exploitation. The detail of these packings are given in the next subsection, while a number of characterisation issues will be developed and discussed in other subsections.

\subsubsection{Numerical Examples: Two Sets of Random Packings}

Two sets of random but periodic particle packings are generated within the domain $[-0.1,1.1] \times$ $[-0.1,1.1]$ with the periodic condition applied to both directions. The first set, or U-set, has particle sizes uniformly distributed within a range; the second set, or G-set, has particle sizes obeying Gaussian distributions with limited minimum and maximum sizes. Each set has four groups each having the particle size range doubled from the previous group, while within each group 10 random packing samples with the same size distribution are generated. The packings and their images at $N=100$ of the $\mathrm{U}$ set are displayed in Figure 5.6; while the 
Table 5.3: Some properties of two sets of random packings

\begin{tabular}{|c|c|c|c|c|c|c|c|c|}
\hline Set & \multicolumn{3}{|c|}{ Uniform Distribution (U-set) } & \multicolumn{4}{|c|}{ Gaussian Distribution (G-set) } \\
\hline Group Name & U1 & U2 & U4 & U8 & G1 & G2 & G4 & G8 \\
\hline Mean Density & .7074 & .7132 & .7143 & .6933 & .7141 & .7156 & .6999 & .7067 \\
\hline Particle No. & 19617 & 4894 & 1216 & 291 & 12950 & 3214 & 791 & 210 \\
\hline$r_{\min }$ & 0.003 & 0.006 & 0.012 & 0.024 & 0.001 & 0.002 & 0.004 & 0.008 \\
$r_{\max }$ & 0.005 & 0.010 & 0.020 & 0.040 & 0.007 & 0.014 & 0.028 & 0.056 \\
\hline$r$ & 0.004 & 0.008 & 0.016 & 0.032 & 0.004 & 0.008 & 0.016 & 0.032 \\
\hline
\end{tabular}

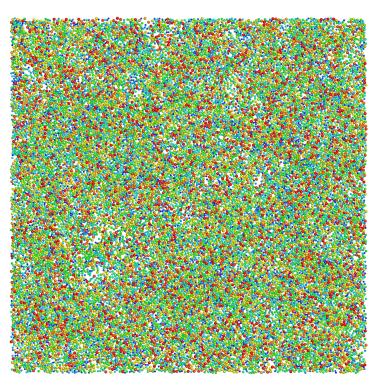

(a) Packing U1

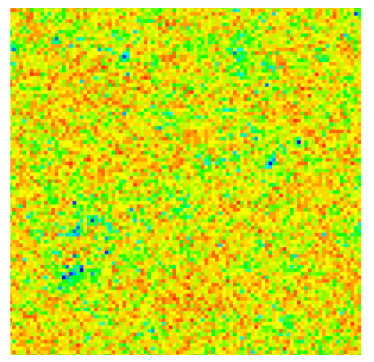

(e) Image U1(N)

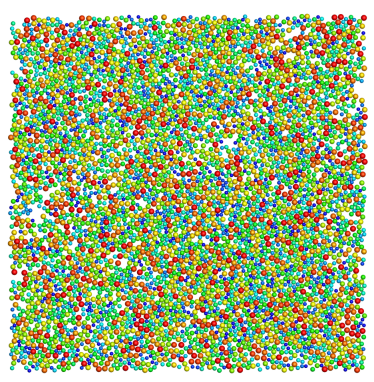

(b) Packing U2

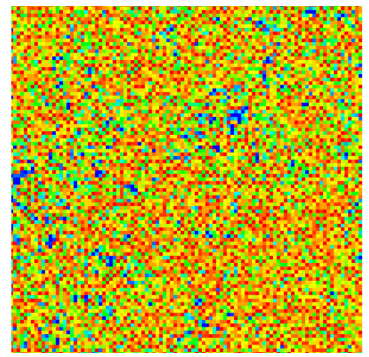

(f) Image U2(N)

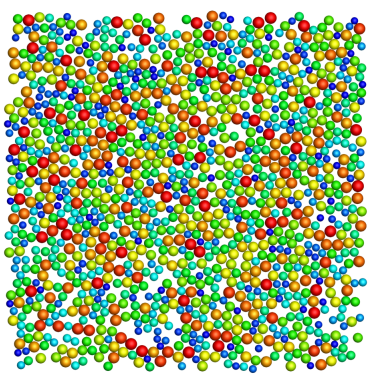

(c) Packing U4

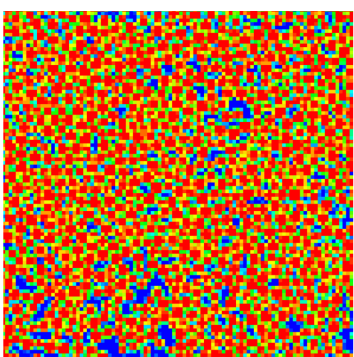

(g) Image U4(N)

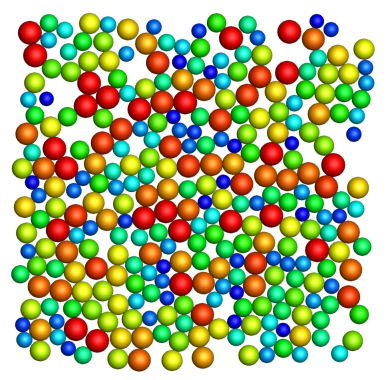

(d) Packing U8

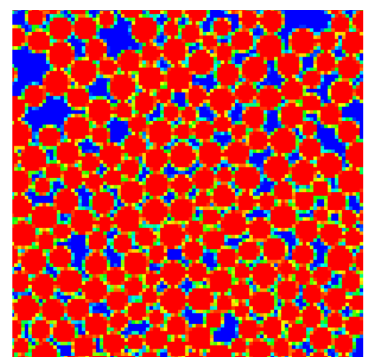

(h) Image U8(N)

Figure 5.6: U-set: Four uniform packing groups in region $[-0.1,1.1] \times[-0.1,1.1](\mathrm{a}-\mathrm{d})$; and their digital images (with $\mathrm{N}=100)$ within region $[0,1] \times[0,1](\mathrm{e}-\mathrm{h})$

packings of the G set are displayed in Figure 5.7.

Table 5.3 lists all the details about the packings up to the group level, including the minimum, maximum and average particle sizes, $r_{\min }, r_{\max }, r$, and the average number of particles in each group. In the U-set, the size distributions of groups U2, U4, U8 are respectively 2, 4, and 8 times of the base group U1. This also applies to the G-set. The average particle size $r$ is the same for the corresponding groups between the two sets: Ui and $\mathrm{Gi}(\mathrm{i}=1,2,4,8)$ have the same $r_{i}$. For the G-set, the standard deviation of the Gaussian distribution for a group is taken to be $\left(r_{\max }-r_{\min }\right) / 2$.

The default analysis window is chosen to be the unit square region $[0,1] \times[0,1]$, so $M=N$. Although the packing density is set to be $\rho=0.7$, the packing density within the analysis window is slightly different from 0.7 , as the window is smaller than the packing region. The mean density for each group is listed in Table 5.3. Because all the mean densities are very close to 0.7 , no significant effect on principal variances is expected. 


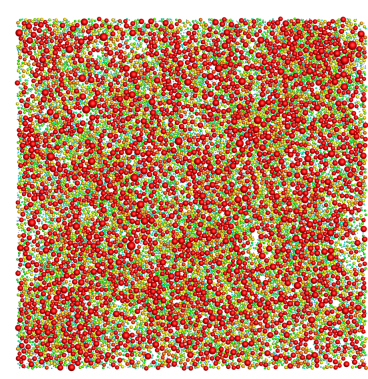

(a) Packing G1

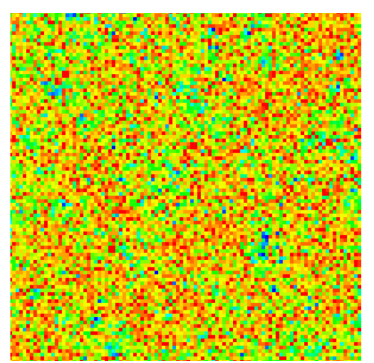

(e) Image G1

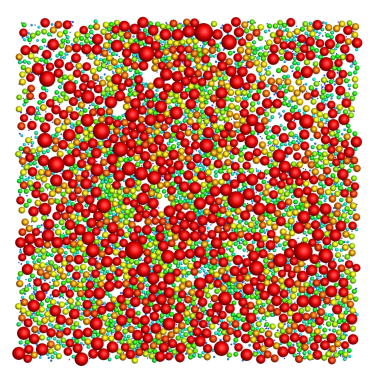

(b) Packing G2

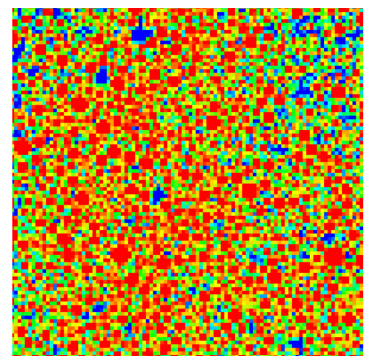

(f) Image G2

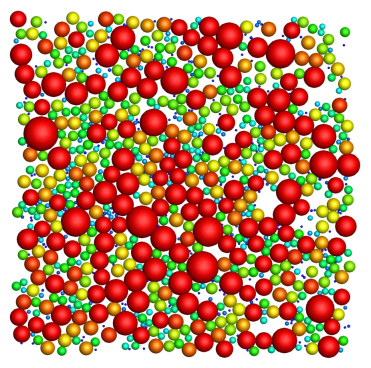

(c) Packing G4

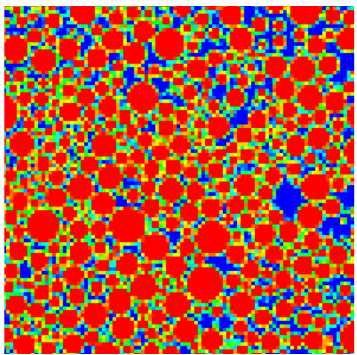

(g) Image G4

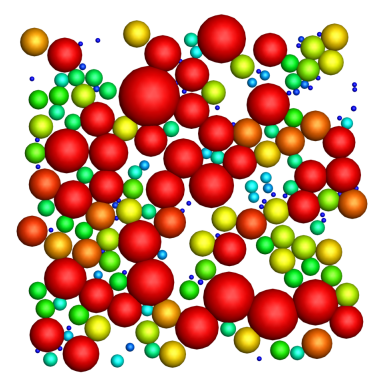

(d) Packing G8

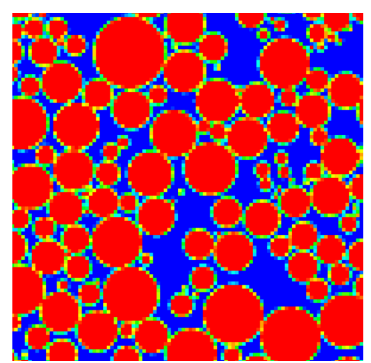

(h) Image G8

Figure 5.7: G-set: Four Gaussian packing groups in region $[-0.1,1.1] \times[-0.1,1.1](\mathrm{a}-\mathrm{d})$; and their digital images (with $\mathrm{N}=100)$ within region $[0,1] \times[0,1](\mathrm{e}-\mathrm{h})$

A column-wise PCA is applied to each sample with required grid resolutions $N$. For each group, the principal variances at a given resolution $N$ are taken to be the average of all the samples in the group.

The first three principal modes of U1(100) and G1(100) are also plotted in Figure 5.8(a) and (b) respectively for illustrative purpose. The randomness/irregularity of the modes are the dominant feature compared to the regular packing case (see Figure 5.5). No further discussion regarding the principal modes will be conducted.

\subsubsection{Dis/Similarity between Packing Samples and Groups}

For each packing group, the PVs of all 10 samples are computed and their averages are taken to be the PVs of the group. For illustrative purpose, the principal variance functions of the 10 samples for groups U1 and G1 at three grid resolutions $N=100,400$, and 1600 are displayed in Figure 5.9. Clearly the PV functions of the 10 samples at each set are located within a narrow band around the group mean value where the maximum difference appears at the leading variances but the difference is much reduced for smaller PVs. This indicates that these samples randomly generated from the same distribution indeed have very similar statistical features.

To quantify the difference, the dissimilarity coefficient of 10 samples in each group are calculated based on the formula (5.25) against their group average for three resolutions: $N=100,400$ and 1600 . The average dissimilarity coefficients of 10 samples in each group for the three resolutions are provided in Table 5.4. The DCs between the corresponding groups, 


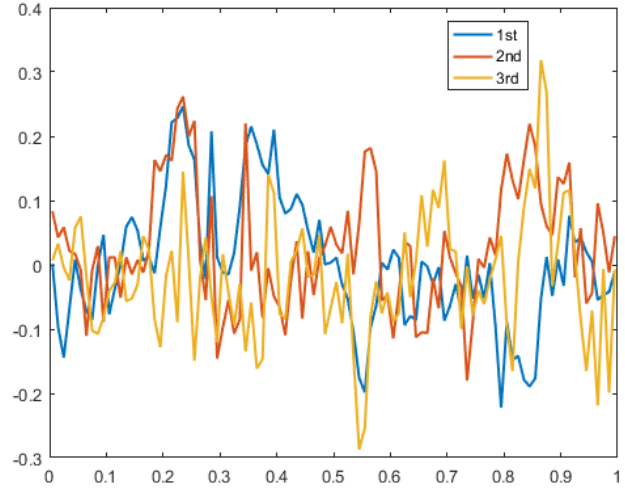

(a) U1(100)

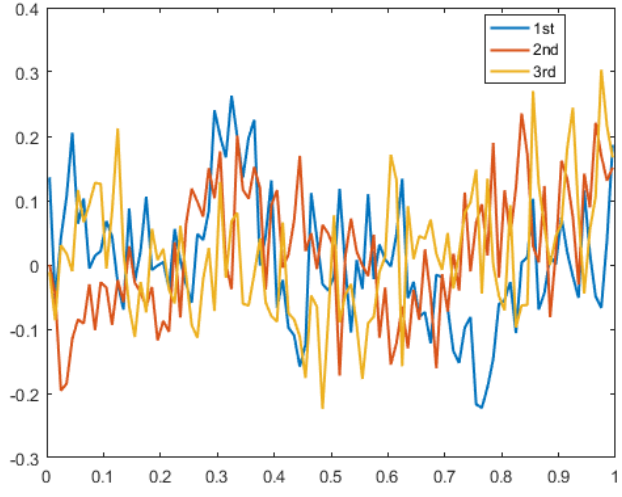

(b) G1(100)

Figure 5.8: First three principal modes of $\mathrm{U} 1$ and G1 at $N=100$

Table 5.4: Average dissimilarity coefficient of each group in two sets of random packings

\begin{tabular}{|c|c|c|c|c||c|c|c|c|}
\hline $\mathrm{N}$ & $\mathrm{U} 1$ & $\mathrm{U} 2$ & $\mathrm{U} 4$ & $\mathrm{U} 8$ & $\mathrm{G} 1$ & $\mathrm{G} 2$ & $\mathrm{G} 4$ & $\mathrm{G} 8$ \\
\hline 1600 & 0.0094 & 0.0196 & 0.0374 & 0.0621 & 0.0160 & 0.0239 & 0.0401 & 0.1301 \\
800 & 0.0101 & 0.0199 & 0.0366 & 0.0599 & 0.0161 & 0.0231 & 0.0371 & 0.1193 \\
400 & 0.0126 & 0.0215 & 0.0372 & 0.0602 & 0.0178 & 0.0238 & 0.0371 & 0.1200 \\
200 & 0.0201 & 0.0261 & 0.0397 & 0.0622 & 0.0223 & 0.0266 & 0.0387 & 0.1235 \\
100 & 0.0378 & 0.0379 & 0.0465 & 0.0662 & 0.0286 & 0.0335 & 0.0435 & 0.1303 \\
\hline
\end{tabular}

Ui-Gi $(\mathrm{i}=1,2,4,8)$, of the two distribution sets are also computed and given in Table 5.5, where their group averaged PVs are used for the calculation.

To visualise the DCs, the sample DCs of the corresponding groups of the two sets for each grid resolution, together with the group DC as a reference value, are all plotted in Figure 5.10. Clearly, dissimilarity reduces when $N$ increases, and in all cases the coefficient seems to converge to a value of around 0.16 , or a similarity index $=84$, regardless of groups.

It may be concluded: 1) 10 samples within each group have very small differences, and the similarity indices of most groups can reach 99; but the difference increases when the number of particles in the packing decreases. 2) The dissimilarity between the corresponding groups of the two distributions are substantially larger than that of the samples within each group, indicating that the PVs can indeed be utilised to effectively classify packings.

Table 5.5: Dissimilarity coefficients between groups in two sets of random packings

\begin{tabular}{|c|c|c|c|c|}
\hline $\mathrm{N}$ & $\mathrm{U} 1-\mathrm{G} 1$ & $\mathrm{U} 2-\mathrm{G} 2$ & $\mathrm{U} 4-\mathrm{G} 4$ & $\mathrm{U} 8-\mathrm{G} 8$ \\
\hline 1600 & 0.1610 & 0.1616 & 0.1835 & 0.1471 \\
800 & 0.1792 & 0.1672 & 0.1892 & 0.1421 \\
400 & 0.2329 & 0.1851 & 0.1970 & 0.1439 \\
200 & 0.3459 & 0.2359 & 0.2153 & 0.1482 \\
100 & 0.5021 & 0.3427 & 0.2634 & 0.1577 \\
\hline
\end{tabular}




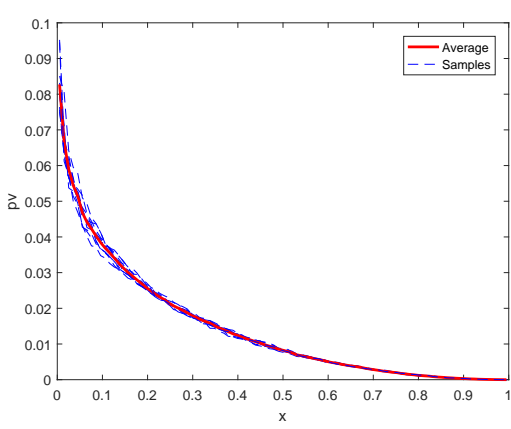

(a) $\mathrm{U} 1(100)$

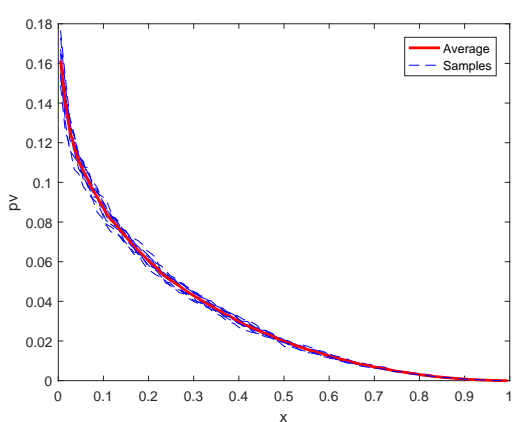

(a) G1(100)

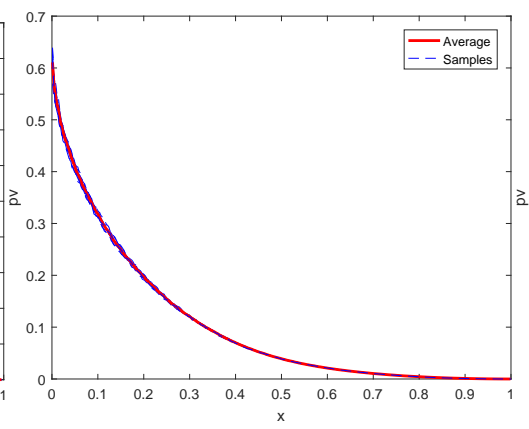

(b) U1(400)

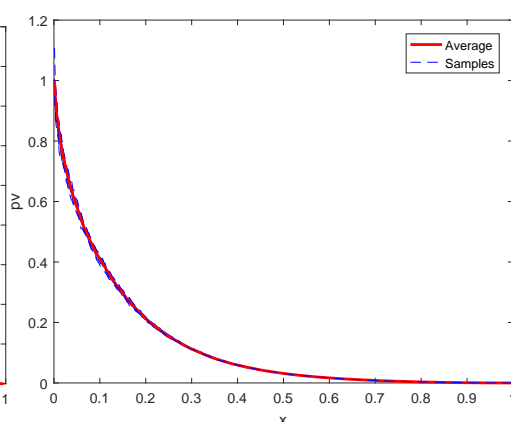

(b) G1(400)

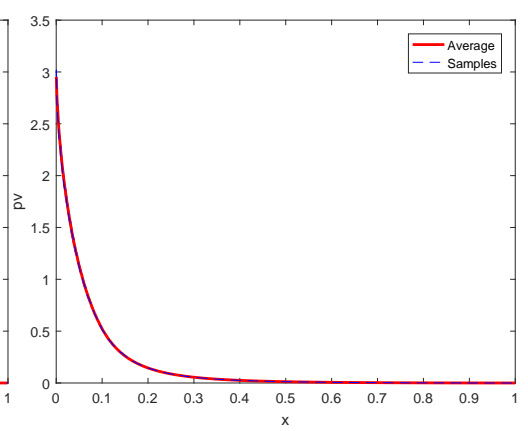

(c) U1(1600)

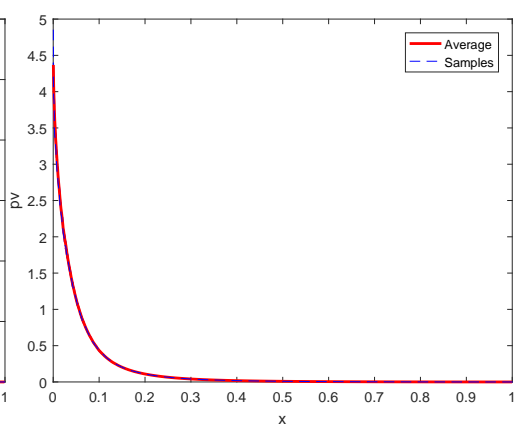

(b) G1(1600)

Figure 5.9: Average principal variance functions of groups U1 and G1 for three resolutions: $\mathrm{N}=100,400,1600$

Principal variances can be employed to exploit the properties of a packing in more detail, such as its spatial uniformity or homogeneity and isotropy.

\subsubsection{Packing Uniformity and Isotropy}

\section{Uniformity}

For one packing, its uniformity in space can be checked by applying a moving analysis window and obtaining the PVs at some selected locations, and then compute the DCs of these PVs by (5.25). A uniformly small DC indicates that the packing may be statistically homogeneous.

Alternatively, if the image $\mathbf{A}_{h}$ of a packing within a large analysis window is given, by selecting smaller analysis windows with the same grid spacing $h$, or by simply selecting sub-matrices/subimages from $\mathbf{A}_{h}$, the spatial uniformity of the packing can also be investigated at a smaller scale. To illustrate this approach, U1(1600) and G1(1600) are used as examples. A number of equally-sized sub-matrix blocks are randomly extracted from the two packing images and PCA is applied to every sub-matrix to obtain its PVs. Three different sizes of sub-matrices $800 \times 800,400 \times 400$, and $200 \times 200$, equivalent to analysis windows of [0.5 $\times 0.5],[0.25 \times 0.25]$, and $[0.2 \times 0.2]$, are considered and their numbers are 10, 20, 40 respectively. For the same sized sub-matrices, their dissimilarity coefficients against the average PVs are evaluated, and the averaged dissimilarity coefficients are also obtained. 

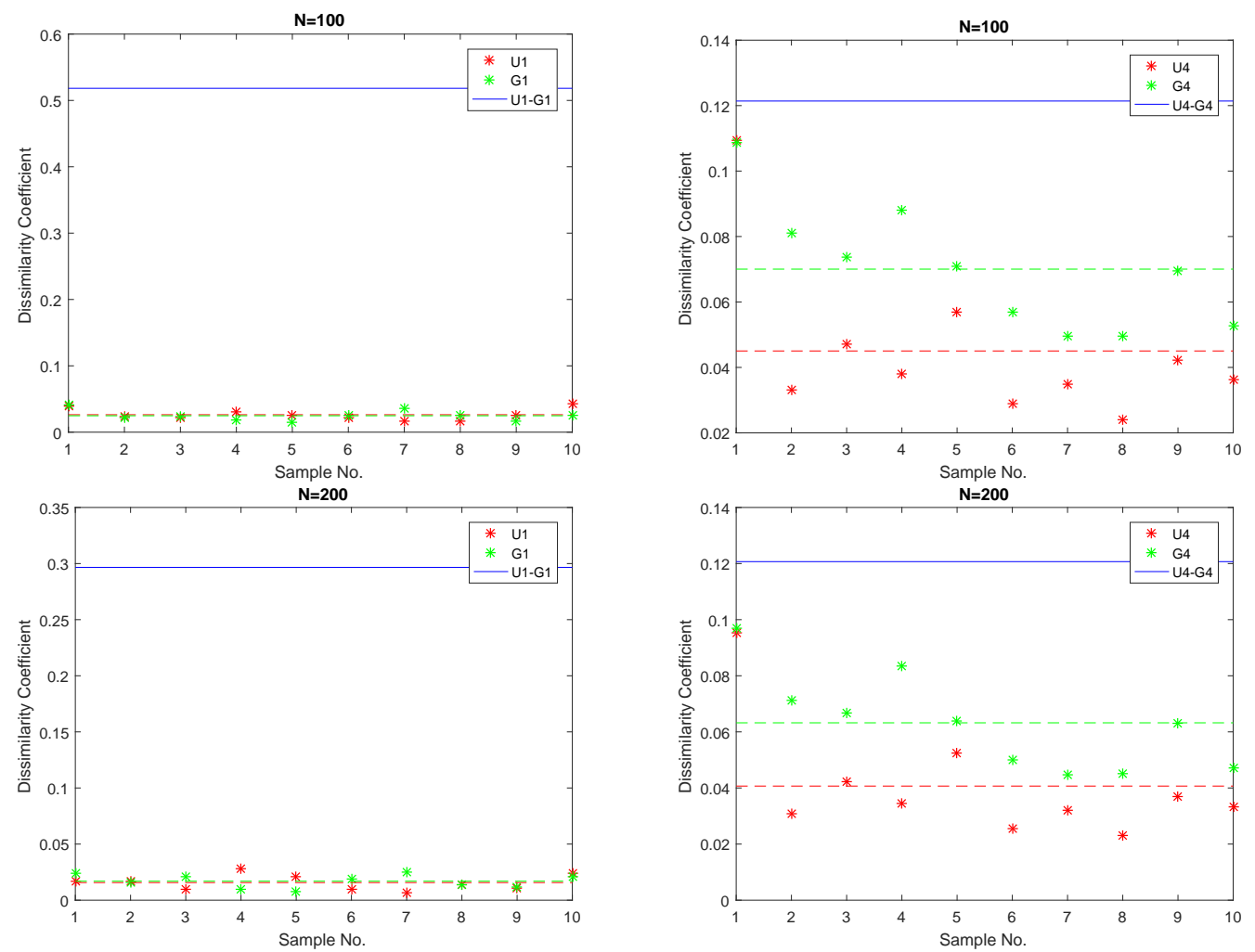

(a) U1, G1 Groups

(b) U4, G4 Groups

Figure 5.10: Dissimilarity coefficients of four packing groups U1, U4, G1 and G4 with four different image resolutions $N=100,200,400$, and 800

Figure 5.11 depicts the average PVs of the three different sized sub-matrices against those of 4 images U1(1600), U4(1600), G1(1600), and G4(1600) respectively. The logarithmic scale for $x$ is used to enlarge the differences around the leading PVs, otherwise almost identical curves are observed at a normal scale. Both dissimilarity coefficients within each sub-matrix group and against the associated whole image are presented in Table 5.6. Furthermore, the individual dissimilarity coefficients of the sub-matrices against the averaged value of each group are displayed in Figure 5.12.

Table 5.6 shows that for U1(1600) and G1(1600), the differences between the submatrices and their associated whole images are generally very small, and no larger than $5 \%$ even with the smallest block size of 200; while for U4(1600) and G4(1600) which have much fewer particles,

Table 5.6: Dissimilarity coefficients of Submatrices of 4 packing images at $N=1600$

\begin{tabular}{|c|c|c|c|c|c|c|}
\hline Comparison & \multicolumn{3}{|c|}{ Within Group } & \multicolumn{3}{c|}{ With the whole image } \\
\hline Submatrix size & 800 & 400 & 200 & 800 & 400 & 200 \\
\hline U1(1600) & 0.012 & 0.037 & 0.050 & 0.010 & 0.009 & 0.014 \\
G1(1600) & 0.015 & 0.029 & 0.052 & 0.009 & 0.022 & 0.022 \\
U4(1600) & 0.054 & 0.095 & 0.167 & 0.038 & 0.069 & 0.109 \\
G4(1600) & 0.140 & 0.194 & 0.221 & 0.139 & 0.221 & 0.318 \\
\hline
\end{tabular}



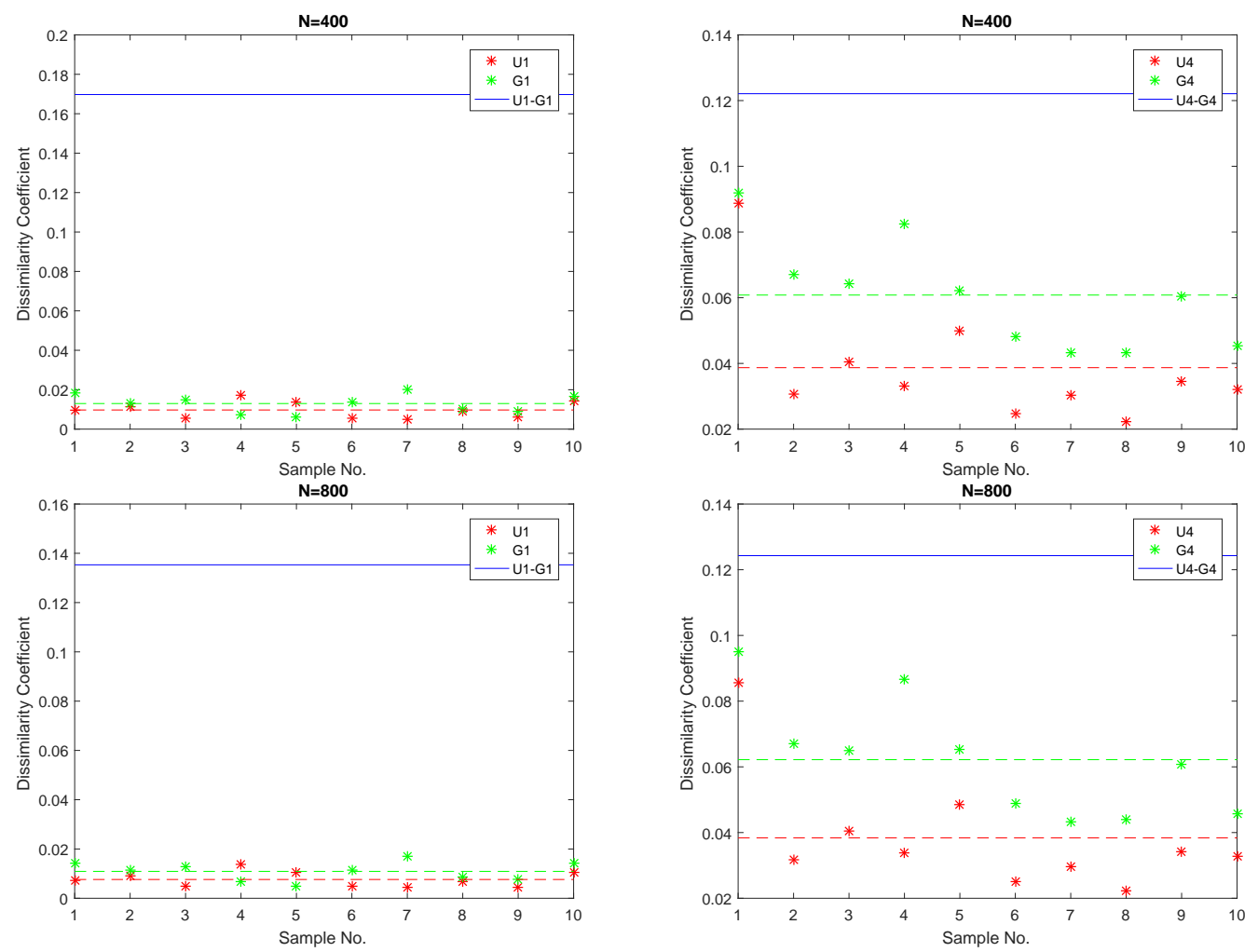

(a) U1, G1 Groups

(b) U4, G4 Groups

Figure 5.10: Dissimilarity coefficients of four packing groups U1, U4, G1 and G4 with four different image resolutions $N=100,200,400$, and 800-Continued

the differences are higher and reach about $20 \sim 30 \%$ for the block size of 200 , indicating that it is more difficult to generate statistically equivalent particle packings with a small number of particles.

It can be seen from Figure 5.12 that the distribution of the dissimilarity coefficient over the entire selection samples for each case is not constant. For U1 and G1 cases, a small level of spatial in-homogeneity exists in the four packings concerned, but for U4 and G4, a large degree of in-homogeneity is observed, as expected.

It is worth in highlighting that the above similarity comparisons between images with different resolutions can not be properly done without using the principal variance function defined earlier.

\section{Isotropy}

By comparing a column-wise and a row-wise PCA to a packing image can reveal if the packing within the analysis window is (an)isotropic in these two directions. A more detailed isotropy check of the packing may be conducted by rotating the analysis window from $0^{\circ}$ to $180^{\circ}$, and applying PCA to each angle, as illustrated by Figure 5.13(a). Then the DCs of the PVs of these rotated packings against their averaged PVs will reveal if the given packing is generally isotropic or not in a broader sense. Take G1 as an example, and choose an analysis window of 


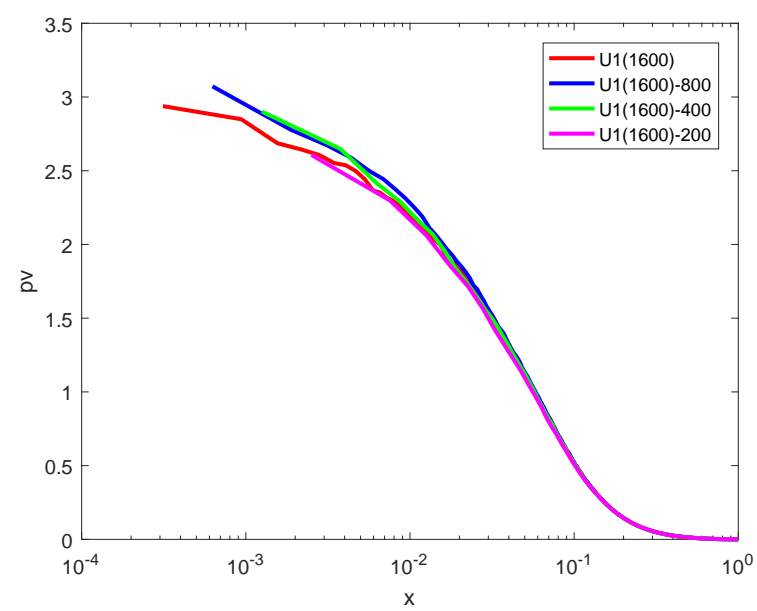

(a) U1(1600)

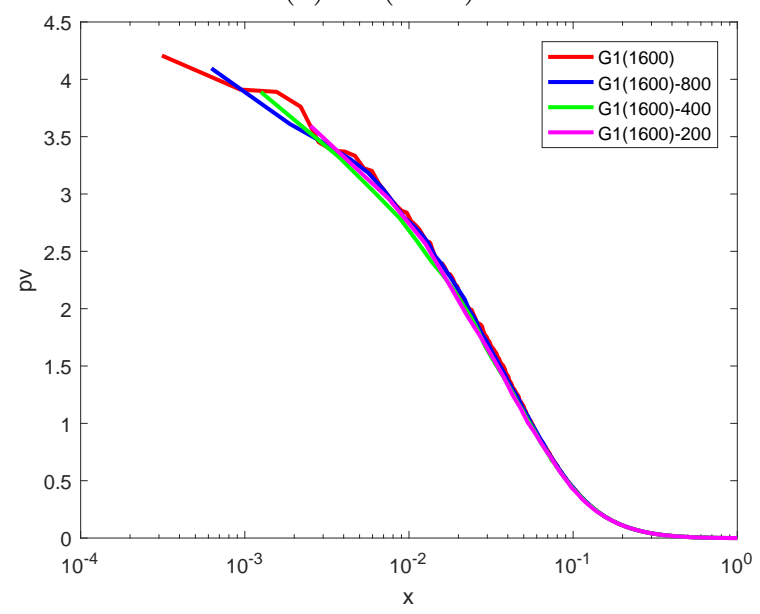

(a) G1(1600)

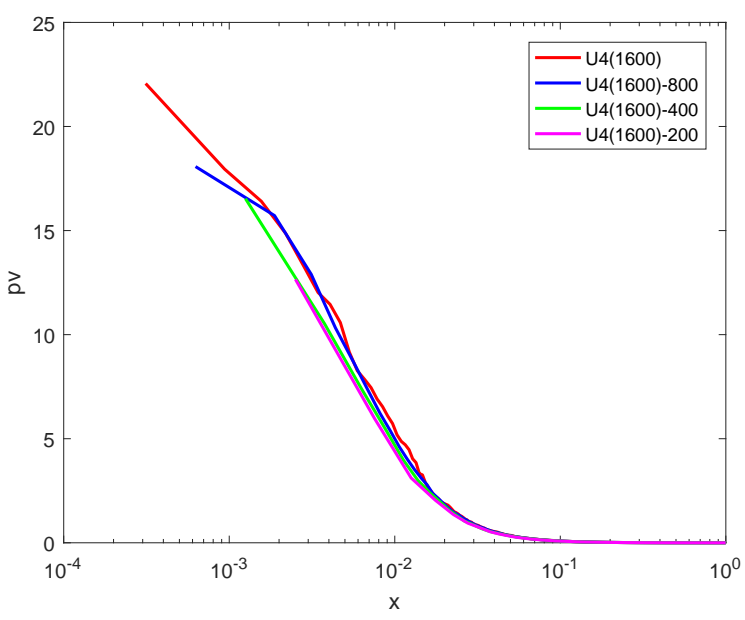

(b) U4(1600)

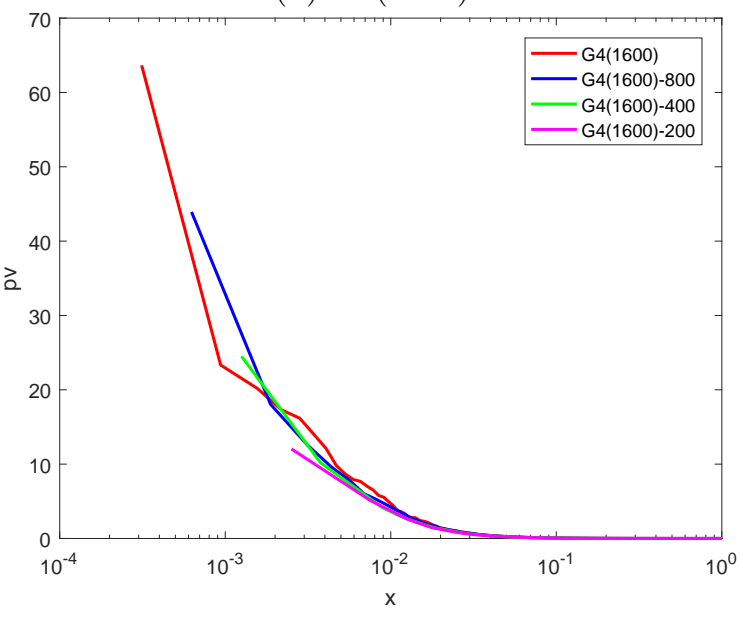

(b) G4(1600)

Figure 5.11: Principal coefficients of four images U1, U4, G1, G4 at N=1600 with three different sub-matrix blocks: $800 \times 800,400 \times 400$, and $200 \times 200$

$[0.5 \times 0.5]$, the DCs of the rotated packings with two resolutions $N=100$ and 400 are displayed in Figure 5.13(b). It indicates that weak isotropy exists for the packing.

In a similar fashion, strong anisotropy of a packing can also be identified by PVs. Figure 5.14(a) shows a packing generated from U4 but some particles being removed from a central strip to make it anisotropic. By applying both a column-wise and a row-wise PCA to the packing image at $N=100$, the two sets of PVs in the two perpendicular directions are attained and displayed in Figure 5.14(b), demonstrating that the strong anisotropy is indeed captured by significant differences in the leading PVs.

\subsubsection{Packing Density Effects}

It is not obvious how packing density affects PVs and more specifically, if two different densities could lead to two very similar PV sets. Such an effect is briefly considered by examples. For each group of U4 and G4, another group of 10 samples is also generated with a smaller packing 


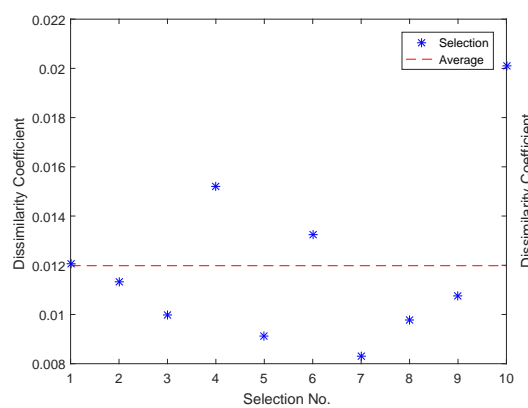

(a) $\mathrm{U} 1-800$

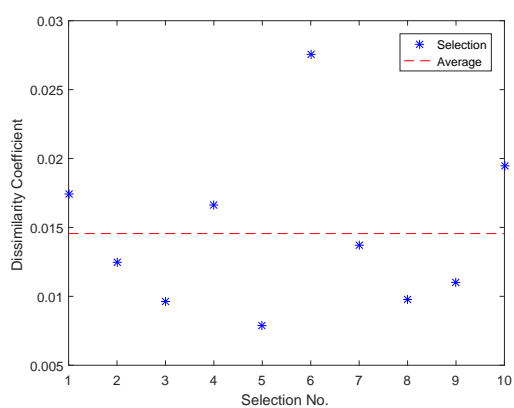

(d) G1-800

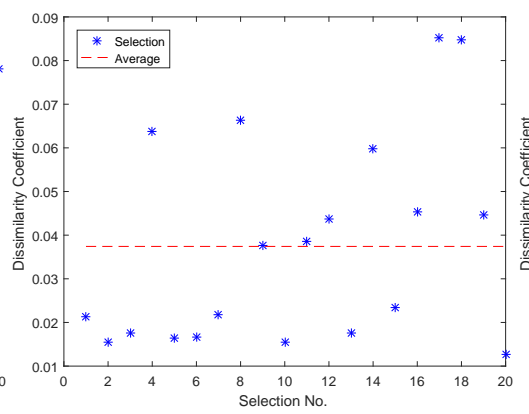

(b) U1-400

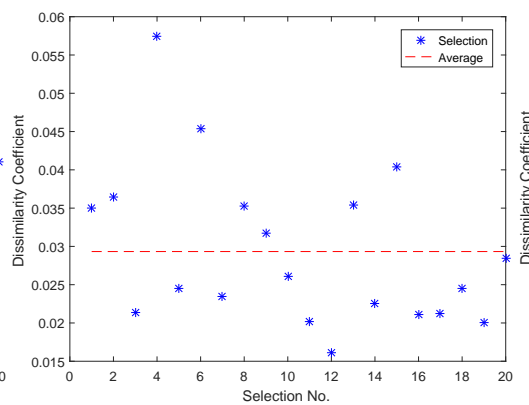

(e) G1-400

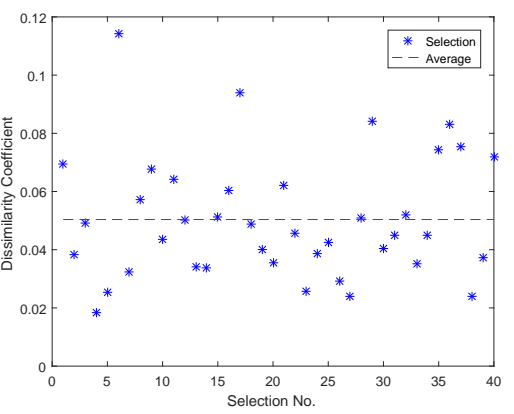

(c) U1-200

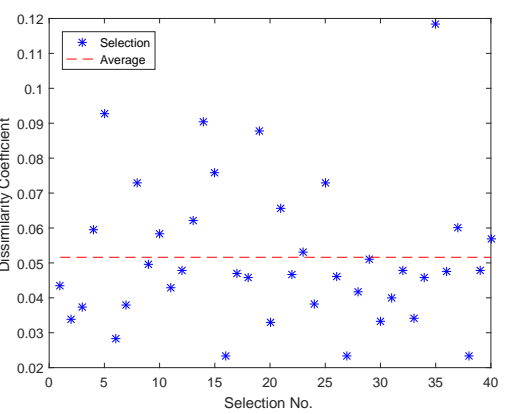

(f) G1-200

Figure 5.12: Dissimilarity coefficient distributions at randomly selected positions of groups U1, G1, U4 and G4 for 3 sizes of sub-matrices: $\mathrm{N}=800,400,200$

Table 5.7: Dissimilarity coefficients of two groups with two packing densities

\begin{tabular}{|c|c|c|c|c|c|}
\hline Group & $\mathrm{N}=1600$ & $\mathrm{~N}=800$ & $\mathrm{~N}=400$ & $\mathrm{~N}=200$ & $\mathrm{~N}=100$ \\
\hline U4 & 0.0659 & 0.0670 & 0.0684 & 0.0714 & 0.0783 \\
\hline G4 & 0.0986 & 0.0976 & 0.0983 & 0.1019 & 0.1106 \\
\hline
\end{tabular}

density of $\rho=0.65$. The dissimilarity coefficients between the groups with different densities for five resolutions $N=1600,800,400,200,100$ are computed and given in Table 5.7, showing around $10 \%$ difference on average. It also appears that reducing the packing density tends to increase the (total and principal) variances. This may be explained by the density-variance relationship (5.4) from which the maximum total variance is reached when $\rho_{\mathcal{A}}=0.5$, i.e. achieve a maximum spatial material variance in the packing. As in the current case, reducing the density from 0.7 to 0.6 should increase the variances in general, leading to increased dissimilarity coefficients. This provides further evidence that PVs can indeed be taken as the signature of a packing which can differentiate packings with different densities.

\subsubsection{Principal Variances via Grid Resolutions}

Many results presented earlier clearly demonstrate a strong dependence of PVs on the grid resolution $N$ or spacing $h$. Generally speaking, both the total variance and the PVs increase with the increase of $N$ or decrease of $h$, but their limits exist. Further investigations reveal 


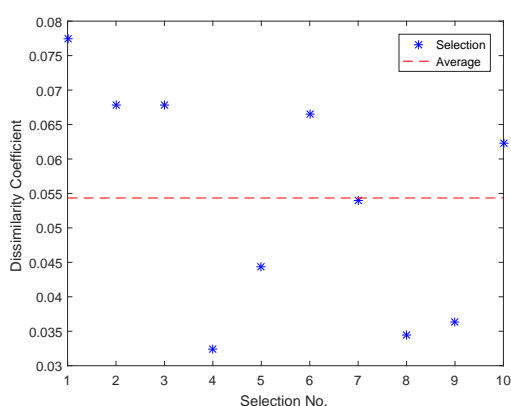

(g) U4-800

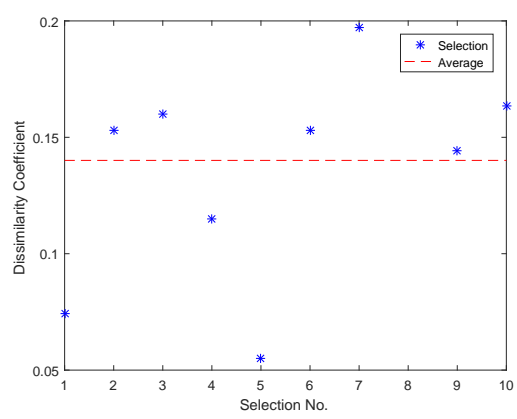

(j) G4-800

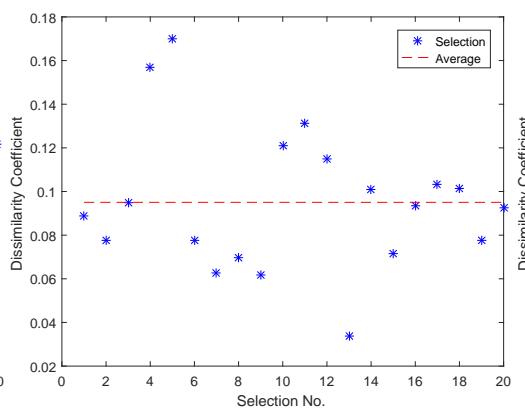

(h) U4-400

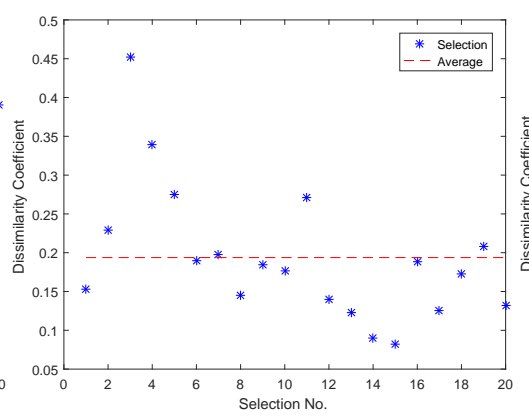

(k) G4-400

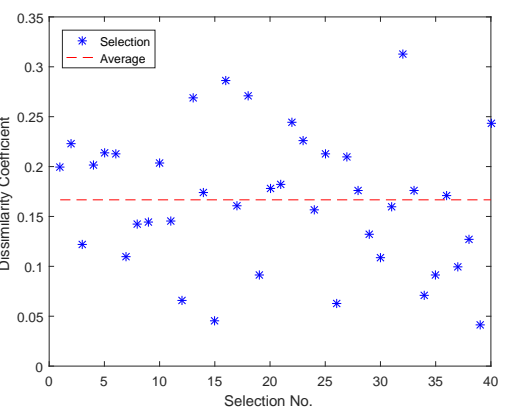

(i) U4-200

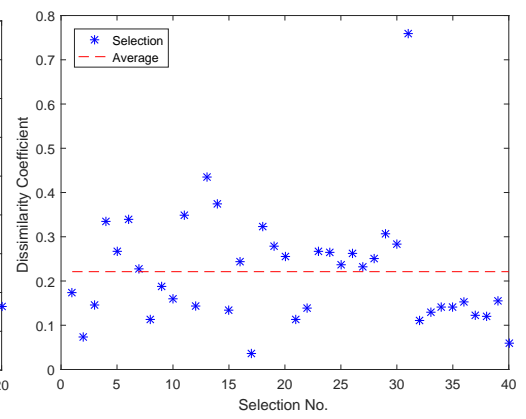

(l) G4-200

Figure 5.12: Dissimilarity coefficient distributions at randomly selected positions of groups U1, G1, U4 and G4 for 3 sizes of sub-matrices: $N=800,400,200$-Continued

that the total variance and the leading PVs converge almost linearly towards the limits as $1 / N$ or $h$ tends to zero. A similar convergent behaviour has already been observed for DCs within samples of the same group, as shown in Table 5.4, and between different groups of the two sets as shown in Table 5.5. Note, however, that these DCs are computed for two packings with the same sized analysis window and grid resolution. While in Section 5.4.3, the comparison is conducted for different sized analysis windows and resolutions but with the same spacing $h$, showing a convergence of PVs as the size of the selected analysis window increases.

\subsubsection{Principal Variances for Scaled Random Packings}

The packing groups within each set considered are deliberately generated to have their size distributions scaled from the base group by a factor of $2^{m}(m=1,2,3)$ so that the relationship between PVs (and DCs) and the scaling factor can be easily established.

The principal variance functions of U2(800), U4(400) and U8(200) against U1(1600) are plotted in Figure 5.15(a) and the corresponding DCs against U1(1600) are also shown; while the PV functions of G2(800), G4(400) and G8(200) against G1(1600), together with their DCs, are displayed in Figure 5.15(b). Notice that the ratio between the average radius and the spacing $r / h$ is kept to be 0.15 for all the cases. The figure clearly indicates that the PVs of U1(1600), U2(800), U4(400) and U8(200) are very similar, with the maximum difference being 


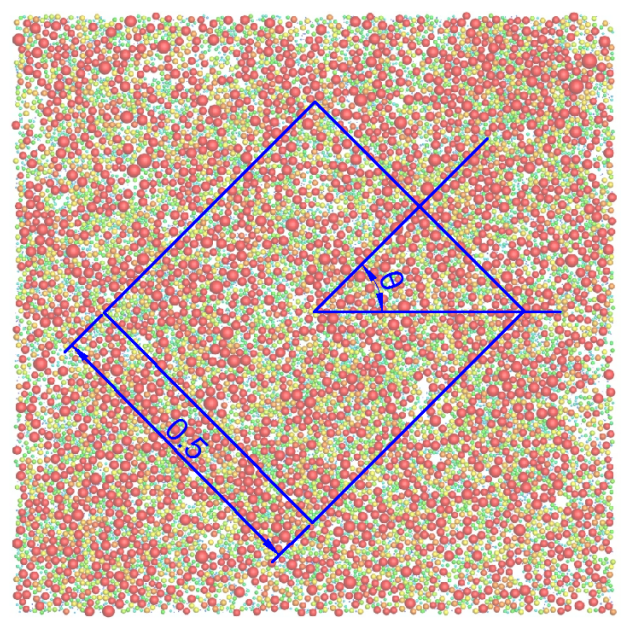

(a) A rotating $0.5 \times 0.5$ analysis window

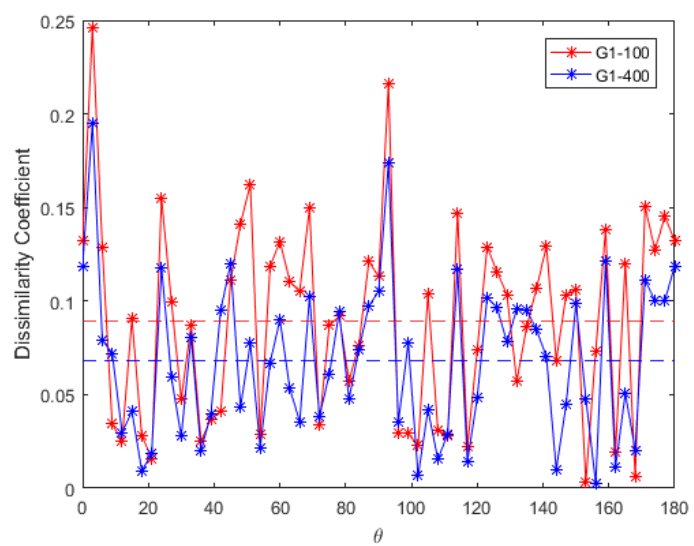

(b) Dissimilarity coefficients for different angles

Figure 5.13: Isotropic check for G1 using a rotating analysis window

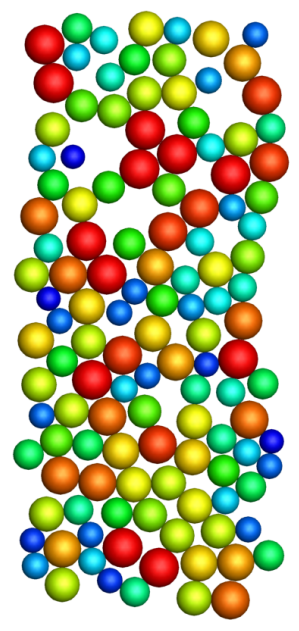

(a) An anisotropic packing
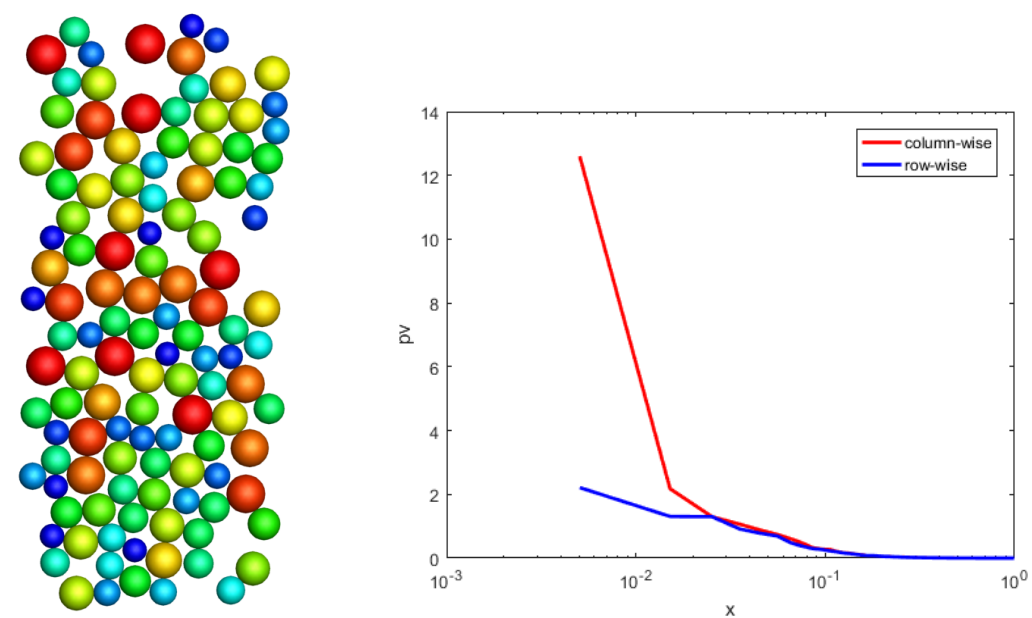

(b) Dissimilarity coefficients for two directions

Figure 5.14: Packing anisotropic checking in two directions

about $10 \%$, or the minimum similar index $=90$. The same applies to G1(1600), G2(800), G4(400) and G8(200). In other words, the PVs of a set of scaled packings may be very close if different resolutions are chosen such that $r * N$ or $r / h$ is a constant.

It is, however, not the case for the regular packings discussed in Section 5.3.3, where similar PVs are obtained for the same grid resolution $N$ or spacing $h$. This apparent contradiction can be explained without offering a rigorous proof as follows.

Packings R4 and R16 are scaled and repetitive versions of R1, making them $100 \%$ correlated. The relationship in their PVs has been fully established in Section 5.3.2. While for random packing U1(1600) or G1(1600), when it is split into $4800 \times 800$ blocks, these sub-matrices are (almost) statistically independent but have very similar PVs, making their PV functions very close to that of U1(1600) or G1(1600), as demonstrated earlier in Figure 5.12. On the other hand, U2(800) or G2(800) is statistically equivalent to a 2-time up-scaled $800 \times 800$ 


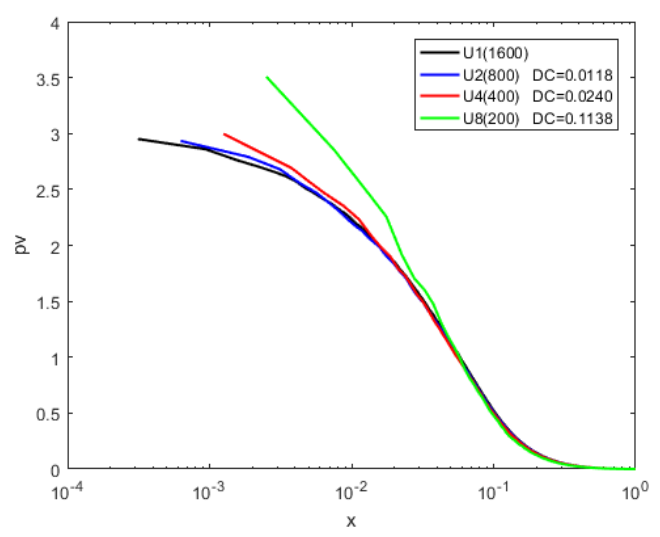

(a) U-set

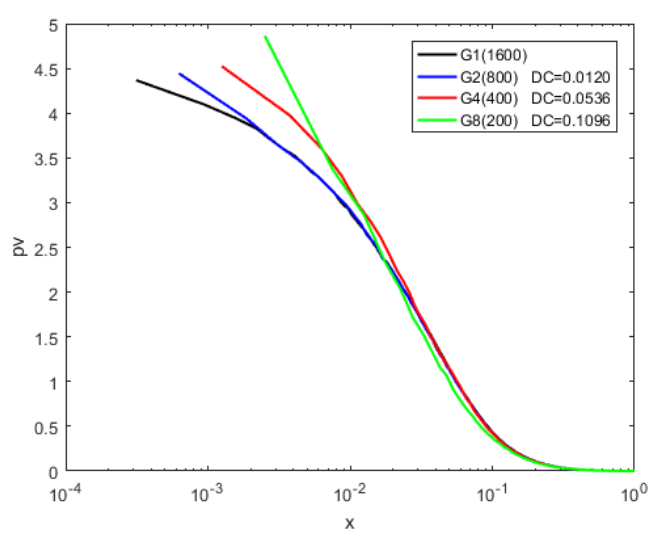

(b) G-set

Figure 5.15: Comparison of the principal variance functions and dissimilarity coefficients of scaled packing groups with different resolutions but with the ratio $r / h$ kept the same

block of U1(1600) or G1(1600), thereby having a similar PV function to the block (refer to Section 5.3.2 for the reason), and therefore also close to U1(1600) or G1(1600).

\subsection{Concluding remarks}

This chapter has proposed a Principal Component Analysis based novel methodology to characterise particle packings. It involves first the digitalisation of a packing into a greyscale image; and then the application of PCA to obtain the principal variances of the image. From comprehensive investigations on the effectiveness of characterising some purposefully generated regular and random packings, it can be concluded that the principal variances are the signature of a packing image. Another important development is the definition of a dissimilarity coefficient or equivalently a similarity index, by which the degree of (dis)similarity of two packing images can be quantitatively compared and evaluated. 


\section{Chapter 6}

\section{Characterising particle packings by PCA-3D}

\subsection{Introduction}

In this chapter, the PCA based packing characterising method is extended from 2D particle packings to 3D cases. Gaussian Quadrature is adopted to obtain the volume matrix representation of a particle packing. Then the digitalised image of the packing is obtained by converting cross-sectional images along one direction to column vectors of the packing image. Comprehensive investigations for several sets of purposefully generated particle packings are conducted to understand relationships of their principal variances with packing features. Differences between two packings with different packing features can be revealed by the principal variances (PV) and dissimilarity coefficient (DC). Furthermore, the values of PV and DC can indicate different levels of effects on packing caused by configuration randomness, particle distribution, packing density and particle size distribution. The uniformity and isotropy of a packing can also be investigated by the PCA based approach. The relationship between the macroscopic response and the DC of a granular system subject to the tri-axial loading is also presented.

\subsection{Principal component analysis}

This section is devoted to the full description of the numerical procedure that is involved in principal component analysis of a packing and how such analysis can be applied to characterise particle packings. The main principals and terminologies adopted in the current work is derived from Section 5.2. 
(a)

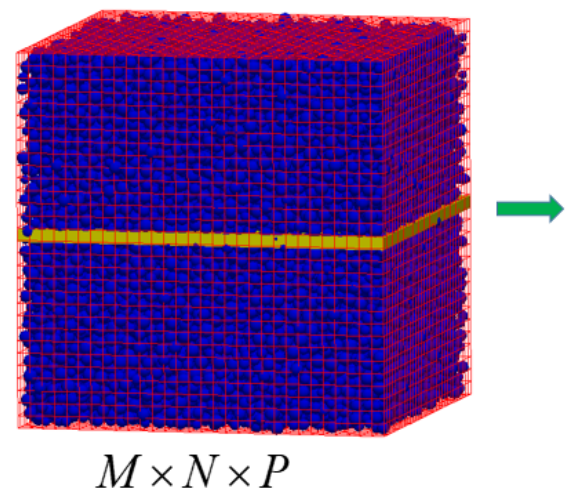

(b)

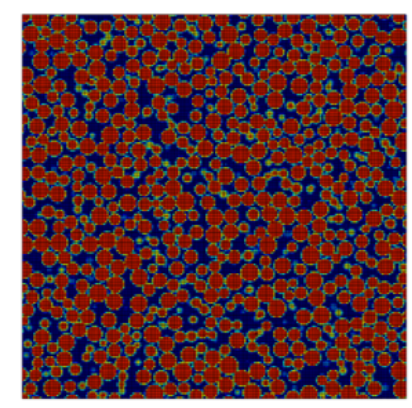

$M \times N$ (c)

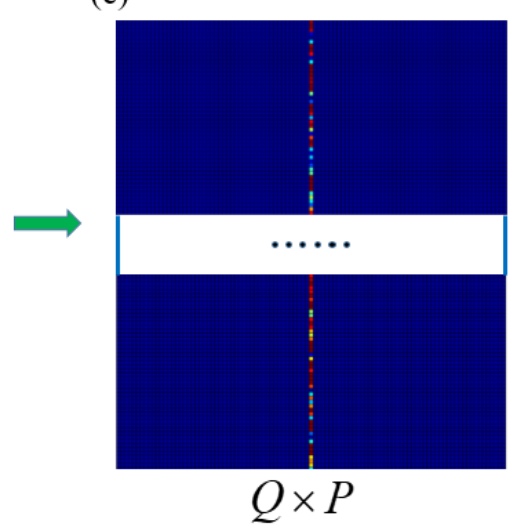

Figure 6.1: A random particle packing and the corresponding digital representation

\subsubsection{Packing digitalisation and formation of packing image}

First consider a particle assembly $\Omega_{p}=\bigcup_{i} \Omega_{i}$ where $\Omega_{i}$ denotes the domain of the $i$-th particle, and arbitrarily choose a cuboid solid region $\mathcal{V}$ of dimensions $L_{x} \times L_{y} \times L_{z}$, termed the analysis region. The region can be divided into a regular grid of $M \times N \times P$ cubic cells with spacing $h=L_{x} / M=L_{y} / N=L_{z} / P$ (Figure 6.1(a)). For a cubic cell at $(i, j, k)$ with the volume denoted as $\mathcal{V}_{i j k}$, compute its average volume covered by particles, or grey-scale as

$$
v_{i j k}=\frac{\left|\Omega_{g} \cap \mathcal{V}_{i j k}\right|}{\left|\mathcal{V}_{i j k}\right|}
$$

where $|\Omega|$ denotes the measure or volume of a domain $\Omega ;|\mathcal{V}|=L_{x} L_{y} L_{z}$; and $\left|\mathcal{V}_{i j k}\right|=h^{3}$. An empty cell with no overlapping with any particle has $v_{i j k}=0$; while a cell fully covered by a particle has $v_{i j k}=1$. A cell partially covered by particles has $v_{i j k}<1$. So in general $v_{i j k} \in[0,1]$. The computation of $v_{i j k}$ is however not straightforward. Different numerical schemes used to evaluate $v_{i j k}$ are discussed below.

The collection of all the average volumes $v_{i j p}$ at the layer $p$ in the $z$-direction (highlighted in Figure 6.1(a)) forms an $M \times N$ matrix, which can be viewed as a digitalised grey-scale representation of the origin 3D packing at the height of $z(p)$ (as shown in Figure 6.1(b)). The matrix is then re-shaped into a vector simply by concatenating the rows into a single vector with $Q=M N$ elements, and placed as the $p$-th column vector in a larger $Q \times P$ matrix $\mathbf{V}_{h}$. By processing all the $P$ layers in the $z$-direction, the resulting matrix $\mathbf{V}_{h}$ can be viewed as a digitalised grey-scale representation of the original packing $\Omega_{g}$, thus is termed as the packing matrix or image, as shown in Figure 6.1(c). 


\subsubsection{Formulations and numerical procedures}

The mean value of the packing matrix $\mathbf{V}_{h}$, i.e. the packing density of the region $\mathcal{V}$, can be computed

$$
\rho_{\mathcal{V}} \equiv \frac{\left|\Omega_{g} \cap \mathcal{V}\right|}{|\mathcal{V}|}=\frac{1}{Q P} \sum_{i=1}^{Q} \sum_{j=1}^{P} v_{i j}
$$

Let $v(\mathbf{x})$ be the material distribution function with $v$ taking the value of 1 for a point located within a particle, and 0 otherwise. It is not difficult to derive that the total variance of a packing in the region $\mathcal{V}$ is related to the packing density by

$$
\sigma_{\mathcal{V}}=\frac{1}{|\Omega|} \int_{\Omega}\left(v-\rho_{\mathcal{V}}\right)^{2} d \Omega=\rho_{\mathcal{V}}\left(1-\rho_{\mathcal{V}}\right)
$$

The total variance of the matrix is defined as

$$
\sigma_{h}=\frac{1}{Q P} \sum_{i=1}^{Q} \sum_{j=1}^{P}\left(v_{i j}-\rho_{\mathcal{\nu}}\right)^{2} \leq \sigma_{\mathcal{V}}
$$

i.e. the total variance of the packing $\sigma_{\mathcal{V}}$ is the upper bound of any packing image.

Let $q_{j}$ be the mean value of the $j$-th column of the packing matrix $\mathbf{V}_{h}$

$$
q_{j}=\frac{1}{Q} \sum_{i=1}^{Q} v_{i j}
$$

By subtraction of its mean from each column vector of $\mathbf{V}_{h}$, the column centralised matrix $\overline{\mathbf{V}}_{P}$ of $\mathbf{V}_{h}$ is obtained as:

$$
\overline{\mathbf{V}}_{P}=\mathbf{V}_{h}-\mathbf{e}_{Q} \mathbf{q}_{P}
$$

where $\mathbf{e}_{Q}$ is an $Q \times 1$ column vector with all its elements being 1's; and $\mathbf{m}_{P}$ is the $1 \times P$ mean value vector $\mathbf{q}_{P}=\left\{q_{j}\right\}$.

Define the covariance matrix of $\overline{\mathbf{V}}_{P}$ as

$$
\mathbf{S}_{P}=\frac{1}{Q} \overline{\mathbf{V}}_{P}^{T} \overline{\mathbf{V}}_{P}
$$

where $\mathbf{S}_{P}$ is a $P \times P$ square matrix. Notice in the above that $Q$ instead of $Q-1$ is used. Further define the column-wise total variance as

$$
\sigma_{P}^{c}=\frac{1}{P} \operatorname{Tr}\left(\mathbf{S}_{P}\right)=\frac{1}{P} \sum_{i=1}^{P}\left(\mathbf{S}_{P}\right)_{i i}
$$

which may be (slightly) different from the total variance $\sigma_{h}$ in general.

By solving the eigenvalue problem of $\mathbf{S}_{P}$, it yields the following matrix decomposition

$$
\mathbf{S}_{P} \mathbf{U}_{P}=\mathbf{U}_{P} \mathbf{D}_{P}
$$


with

$$
\mathbf{D}_{P}=\mathbf{U}_{P}^{T} \mathbf{S}_{P} \mathbf{U}_{P}, \quad \mathbf{U}_{P}^{T} \mathbf{U}_{P}=\mathbf{I}_{P}
$$

where the diagonal matrix $\mathbf{D}_{P}=\operatorname{diag}\left\{d_{i}\right\}$ contains all the eigenvalues $d_{i}$ in descending order, which are termed the principal variances $(\mathrm{PVs})$; and $\mathbf{U}_{N}=\left\{\mathbf{u}_{i}\right\}$ are the orthonormal vectors, termed the principal modes. As $\overline{\mathbf{V}}_{P}$ is column centralised, $\mathbf{S}_{P}$ is a semi-positive definite matrix with at least one zero principal variance. It is also well known that the sum of the PVs and the total column-wise variance is related by

$$
\frac{1}{P} \sum_{i=1}^{P} d_{i}=\sigma_{P}^{c}
$$

$\mathbf{S}_{P}$ can be recovered from the principal variances and modes as

$$
\mathbf{S}_{P}=\mathbf{U}_{P} \mathbf{D}_{P} \mathbf{U}_{P}^{T}=\sum_{i=1}^{P-1} d_{i} \mathbf{u}_{i} \mathbf{u}_{i}^{T}
$$

The column-wise total variance $\sigma_{P}^{c}$, the mean value vector $\mathbf{q}_{P}$, the principal variance matrix $\mathbf{D}_{P}$ and the corresponding modes $\mathbf{U}_{P}$ form a unique set $\mathcal{C}_{\mathcal{P}}$, termed the column-wise characteristic set, that fully determines the packing image.

$$
\mathcal{C}_{\mathcal{P}}=\left\{\sigma_{P}^{c}, \mathbf{q}_{P}, \mathbf{D}_{P}, \mathbf{U}_{P}\right\}
$$

As the PVs and the column-wise total variance is related by (6.10), and the total variance (and also the column-wise variance) is related to the density (see (6.3)), the PVs play a dominant role to characterise a packing image and therefore can be viewed as the signature of the packing.

\subsubsection{Different Schemes to compute the volume average of each cubic cell}

The most time consuming part of the above proposed procedure is the evaluation of the density, or the particle covered volume of a cubic cell (6.1). In 2D cases, the covered area of a square cell by circular particle(s) may be computed analytically depending on their overlapping situations which can be classified by the number of grid nodes contained in the circle(s), while it is difficult to follow a similar procedure to obtain the exact overlap volume between spherical particle(s) and a cubic cell due to the complexity of the contact situations in $3 \mathrm{D}$ cases. Two different computational schemes are therefore adopted to numerical compute the volume average of each cubic cell.

The first scheme, termed the area-based, is derived from the method used in the 2D case which can determine the overlap area between a circle and a square cell. The 3D problem can be reduced to the $2 \mathrm{D}$ one by integrating the overlap area in the $x-y$ plane along the $z$-direction. Gaussian Quadrature is implemented to numerically evaluate the integration. The volume 
Table 6.1: The overall packing density of three regular packings computed by different computational schemes

\begin{tabular}{|c|c|c|c|c|c|c|c|c|c|}
\hline \multirow{2}{*}{$\mathrm{N}$} & \multicolumn{3}{|c|}{ R1 } & \multicolumn{3}{c|}{ R8 } & \multicolumn{3}{c|}{ R64 } \\
\cline { 2 - 10 } & 1-Point & 3-Point & Area & 1-Point & 3-Point & Area & 1-Point & 3-Point & Area \\
\hline 25 & 0.5259 & 0.5231 & 0.5236 & 0.5212 & 0.5230 & 0.5232 & 0.5212 & 0.5232 & 0.5235 \\
\hline 50 & 0.5260 & 0.5238 & 0.5236 & 0.5259 & 0.5231 & 0.5236 & 0.5212 & 0.5230 & 0.5232 \\
\hline 100 & 0.5240 & 0.5236 & 0.5236 & 0.5260 & 0.5238 & 0.5236 & 0.5259 & 0.5231 & 0.5236 \\
\hline
\end{tabular}

average of a cubic cell of side $h$ and centred in a local coordinate system is obtained by

$$
v_{i j k}=\int_{-h / 2}^{h / 2} a_{i j}(z) d z=\sum_{l=1}^{n} w_{l} a_{i j}\left(z_{l}\right)
$$

where $a_{i j}(z)$ is the average area covered by circles at $z$, and $z_{l}$ and $w_{l}$ are the position and corresponding weight of a Gaussian point.

The second scheme, termed the point-based, simplifies the problem by checking whether a point is inside a particle or not. A certain number of points are selected in each cubic cell. Two values 0 (point outside the particle) or 1 (inside the particle) can be assigned to each point. Gaussian Quadrature is again applied in this scheme to select the position and weight of each point in the cubic cell. The volume average of each cubic cell is the integration of the point values. Using $n$ Gaussian points in each directions gives rise to the formula

$$
v_{i j k}=\sum_{l=1}^{n} \sum_{s=1}^{n} \sum_{t=1}^{n} w_{l s t} v\left(x_{l}, y_{s}, z_{t}\right)
$$

where $w_{l s t},\left(x_{l}, y_{s}, z_{t}\right)$ and $v\left(x_{l}, y_{s}, z_{t}\right)$ are the weight, coordinates and material distribution value of each Gaussian point.

Several numerical tests are conducted to compare the above two schemes. Three regular packings are generated in a unit cubic region where there are respectively 1, 8 and 64 equalsized particles in the packings that are labelled as R1, R8 and R64 respectively. It is obviously that the three packings have the same overall packing density $\rho=3 / 4 \pi / 8=0.5236$. The region is then divided into a regular grid of $N \times N \times N$ cubic cells, and three resolutions $N=25,50,100$ are considered. The packing density can also be computed by (6.2). In the first scheme (area-based), the number of sections along the $z$-direction in each cubic for integrating is 3 which is able to obtain sufficiently accurate results. In the second scheme, two different numbers of Gaussian integration points $n=1$ and 3 are tested. Table 6.1 provides the overall packing densities of the three regular packings for different grid resolutions computed by the two computational schemes, while the first principal variances of the three packings with different resolutions are listed in Table 6.2. These results indicate that the two computational schemes can have a similar accuracy when the number of Gaussian points used is reasonably large. 
Table 6.2: The first principal variances of three regular packings computed by different computational schemes

\begin{tabular}{|c|c|c|c|c|c|c|c|c|c|}
\hline \multirow{2}{*}{$\mathrm{N}$} & \multicolumn{3}{|c|}{ R1 } & \multicolumn{3}{c|}{ R8 } & \multicolumn{3}{c|}{ R64 } \\
\cline { 2 - 11 } & 1-Point & 3-Point & Area & 1-Point & 3-Point & Area & 1-Point & 3-Point & Area \\
\hline 25 & 11.715 & 11.623 & 11.587 & 12.043 & 11.187 & 11.140 & 12.043 & 9.817 & 9.781 \\
\hline 50 & 23.291 & 23.434 & 23.450 & 23.401 & 23.217 & 23.146 & 24.056 & 22.347 & 22.253 \\
\hline 100 & 47.026 & 47.053 & 47.054 & 46.567 & 46.853 & 46.885 & 46.788 & 46.420 & 46.279 \\
\hline
\end{tabular}

\subsubsection{Properties of repetitive packings}

In Section 5.3 for $2 \mathrm{D}$ cases, the relationship between packings with repetitive, periodic and symmetric natures has been established, and it is theoretically derived that for a $m$-repetition packing, $1 / m$ of the principal variances will be $m$ times those of the basic structure. This conclusion is also valid for $3 \mathrm{D}$ cases. Take the previous three regular packings as an example: Packing $\mathrm{R} 1$ can be regarded as the basic structure, $\mathrm{R} 8$ is a 2-repetition packing of $\mathrm{R} 1$, and $\mathrm{R} 64$ is a 2-repetition packing of R8 and a 4-repetition packing of R1. From the first principal variances of the three regular packings $\mathrm{R} 1, \mathrm{R} 8$ and $\mathrm{R} 64$ given in Table 6.2, it is evident that, regardless of the integration scheme used, the first PV of R64 with N=100 is about twice of that of $\mathrm{R} 8$ with $\mathrm{N}=50$ which itself is also about twice of that of $\mathrm{R} 1$ with $\mathrm{N}=25$. Note that different resolutions $\mathrm{N}$ are used for different packings to ensure that a constant relative resolution is maintained for the comparison. It can also be observed that the accuracy of the computational scheme used does not influence the relative relationship of two packings.

\subsubsection{Analysis Region and Formation of Packing Matrix}

The analysis region of a packing is not necessarily a cuboid as is assumed earlier. In fact the analysis region can be, in general, a prism which has the same cross-section along one direction (defined as the $z$-direction). Then each section image can be reshaped to a vector with the same number of elements which forms one column of the final analysis matrix $\mathbf{V}_{h}$. For instance, the analysis region could be a cylinder and the cross section along the $z$-direction is a disc as shown in Figure 6.2. Only the elements in the circular area are selected to construct the column vector of the packing image matrix.

Alternative to representing each section image as a column vector in the final image matrix, all the pixels with the same $(x, y)$ coordinates along the $z$-direction can instead be represented by a column vector, resulting in an different packing matrix which is nothing but $\mathbf{V}_{h}^{T}$, the transpose of the original image matrix $\mathbf{V}_{h} . \mathbf{V}_{h}$ and $\mathbf{V}_{h}^{T}$ may have different principal variances in general, but both can be used to characterise particle packings. In the following discussions, $\mathbf{V}_{h}$ is assumed. 

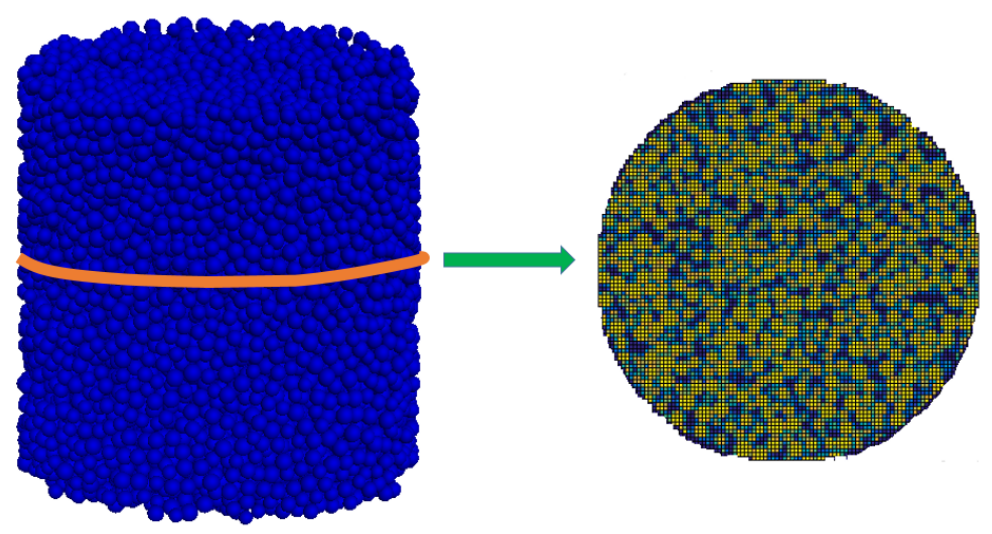

Figure 6.2: A cylindrical analysis region with a circular cross section image

\subsubsection{Evaluation of main principal variances}

The computational cost associated with the solving eigen-value problem (Equation (6.9)) may be high particularly when the resolution $P$ (in the $z$-direction) is large. One option to reduce this cost is to evaluate only a specified number of main principal variances, as will be demonstrated below that the principal variances with largest values play a more dominant role in characterising packings and qualitatively comparing different packings using the (dis)similarity coefficient/index defined earlier.

Note from the definition of the principal variance function that compares images with different resolutions (in the z-direction), the same proportion of the selected number of principal variances over the resolutions should be specified.

\subsection{Packing Characterisation}

This section is devoted to illustrating how the principal variance function and dissimilarity coefficient defined in the previous section can be applied to quantitatively characterise the features of different packings. As the same relative relation of two packings can be obtained by different volume average computational schemes, only the results obtained by the area-based integration scheme are shown below. Several packing samples to be used are given in the next subsection, while a number of characterisation issues will be discussed in later subsections.

\subsubsection{Numerical samples}

Two sets of random particle packings are generated within a unit cubic domain $[-0.5,-0.5,-$ $0.5] \times[0.5,0.5,0.5]$ with the periodic condition applied to all three directions. The first set, termed U-set, has particle sizes uniformly distributed within a range; the second set, termed G-set, has particle sizes obeying a Gaussian distribution with limited minimum and maximum values. Each set has three groups of packing with different particle sizes or densities, while within each group, 10 random packing samples with the same packing properties are generated. 
Table 6.3: Properties of random packings: U-set and G-set

\begin{tabular}{|c|c|c|c|c|c|c|}
\hline Set & \multicolumn{2}{|c|}{ Uniform Distribution (U-set) } & \multicolumn{3}{c|}{ Gaussian Distribution (G-set) } \\
\hline Group Name & U1 & U2 & UL2 & G1 & G2 & GL2 \\
\hline Mean Density & 0.6038 & 0.6062 & 0.5664 & 0.6026 & 0.6053 & 0.5673 \\
\hline Particle No. & 16804 & 2094 & 1939 & 14770 & 1841 & 1701 \\
\hline$r_{\min }$ & 0.015 & 0.030 & 0.030 & 0.015 & 0.030 & 0.030 \\
$r_{\max }$ & 0.025 & 0.050 & 0.050 & 0.025 & 0.050 & 0.050 \\
\hline$r$ & 0.020 & 0.040 & 0.040 & 0.020 & 0.040 & 0.040 \\
\hline
\end{tabular}

Table 6.4: Packing Properties of the L-set

\begin{tabular}{|c|c|c|c|c|c|c|}
\hline Layer & 1 & 2 & 3 & 4 & 5 & 6 \\
\hline$r_{\min }$ & 0.010 & 0.020 & 0.030 & 0.040 & 0.050 & 0.060 \\
$r_{\max }$ & 0.015 & 0.030 & 0.045 & 0.060 & 0.075 & 0.090 \\
\hline$r$ & 0.0125 & 0.025 & 0.0375 & 0.050 & 0.0625 & 0.075 \\
\hline
\end{tabular}

The packing properties used are listed in Table 6.3, and one generated packing sample for each group is illustrated in Figure 6.3. In the U-set, groups U1 and U2 have the same density of 0.6 but the particle size distribution range of U2 is double of U1; groups U2 and UL2 have the same particle size range but different densities with 0.60 for U2 and 0.57 for UL2. The same goes for the groups in the G-set.

The third set, or L-set, is a group of layered particle packings randomly generated within the same domain. Each packing is divided into six layers along the $z$-direction, having particle sizes obeying the uniform distribution within each layer. The particle size distribution ranges of the layers form an arithmetic sequence. The packing density is 0.6 which is the same as those of U1, U2, G1 and G2. The packing properties are given in Table 6.4 and one packing sample is displaced in Figure 6.4.

The principal component analysis is applied to each sample of the U-set, G-set and L-set.

\subsubsection{Different effects on packing samples}

\section{Packing randomness effects}

For each group of U1, U2, G1 and G2, 10 random packing samples are generated with the same packing properties. It is obvious that the packing configurations of these samples in each group are statistically different, but the difference can hardly be evaluated by existing conventional methodologies. As will be shown below, the currently proposed novel PCA based approach has the capability of measuring the effects caused by the randomness of packing configurations.

For each packing group, the PVs of all 10 samples are computed and their averages are taken to be the PVs of the group. The principal variance functions of the 10 samples for groups U1 


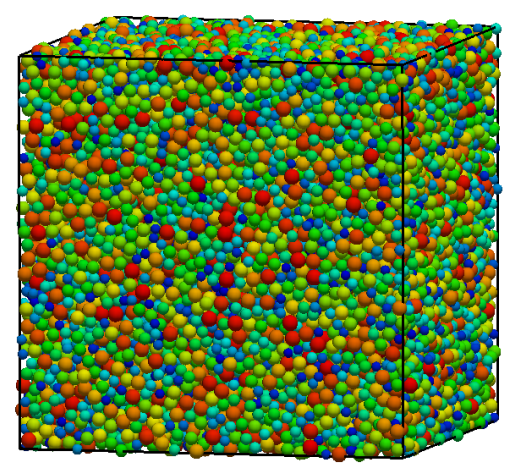

(a) Packing U1

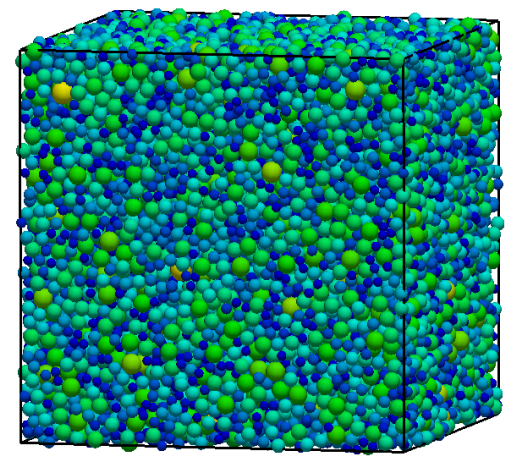

(d) Packing G1

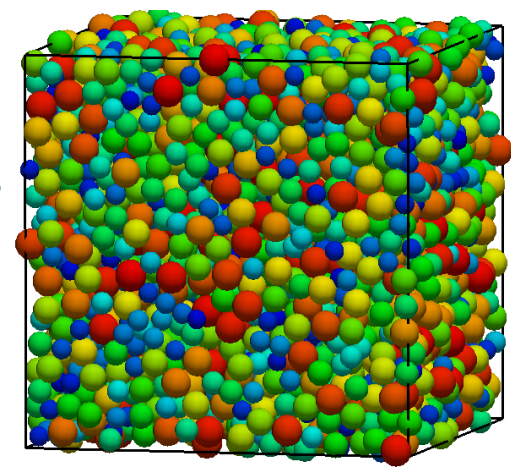

(b) Packing U2

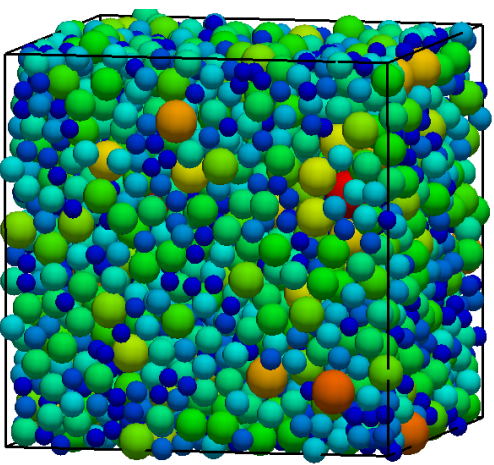

(e) Packing G2

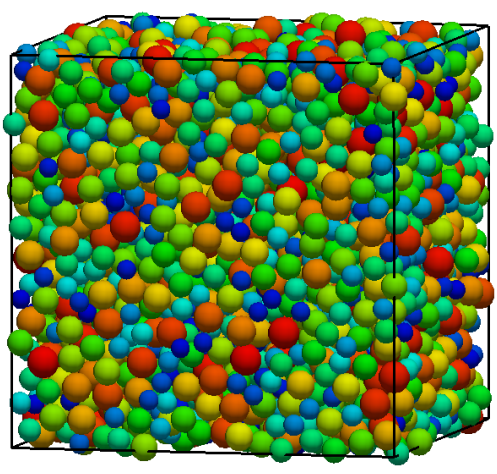

(c) Packing UL2

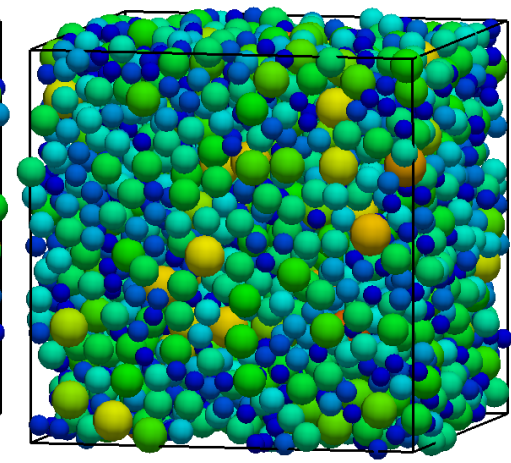

(f) Packing GL2

Figure 6.3: Sample Packings of both U-set and G-set

Table 6.5: Average dissimilarity coefficients of each group in U-set and G-set

\begin{tabular}{|c|c|c||c|c|}
\hline P & U1 & U2 & G1 & G2 \\
\hline 100 & 0.0064 & 0.0119 & 0.0070 & 0.0123 \\
200 & 0.0061 & 0.0114 & 0.0062 & 0.0117 \\
\hline
\end{tabular}

and G1 at two grid resolutions $M(=N=P)=100,200$ are displayed in Figure 6.5. Clearly the PV functions of the 10 samples in each group are located within a narrow band around the group mean value. This indicates that these randomly generated samples from the same particle size distribution indeed have very similar statistical features.

To quantify the difference, the dissimilarity coefficient of 10 samples in each group are calculated based on the formula (5.25) against their group average for two resolutions: 100 and 200. The dissimilarity coefficients of groups U1 and G1 are displayed in Figure 6.6 and the group average of the 10 samples are provided in Table 6.5. As Figure 6.5 visually shows a minor difference among PVs functions of samples in each group, which is confirmed by very small dissimilarity coefficients in Table 6.5, it can be concluded that the effects of the particle distribution randomness is indeed insignificant. It has also been found that the difference decreases when the number of particles in the packing increases. 


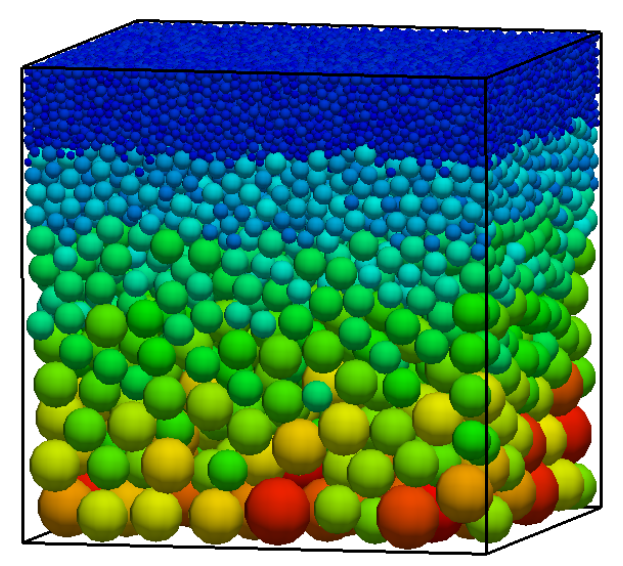

Figure 6.4: A sample packing of the L-set

\section{Particle distribution effects}

The effects of different particle distributions on packings can be illustrated by the comparison between the corresponding groups in both U-set and G-set. For each packing group, the average PVs are taken to be the PVs of the group. The principal variance functions of groups U1, U2, G1 and G2 at two grid resolutions $P=100$ and 200 are displayed in Figure 6.7. To quantify the difference caused by different particle distributions, the dissimilarity coefficients of U1-G1 and U2-G2 are provided in Table 6.6. The dissimilarity between the corresponding groups of the two distributions are larger than that of the samples within each group. In other words, the particle distribution has a more significant influence on the packing features than the packing configuration randomness.

The density profiles of the U1 packing for different resolutions are also presented in Figure 6.8, clearly indicating that the profile is strongly dependent of the resolution $N$, while the general shapes of the PV distribution remain very similar for different $N$, as shown in Figure 6.7.

Table 6.6: Dissimilarity coefficients between different groups

\begin{tabular}{|c|c|c||c|c||c|c|}
\hline P & \multicolumn{2}{|c||}{ Particle distribution } & \multicolumn{2}{c||}{ Packing density } & \multicolumn{2}{c|}{ Particle size } \\
\hline & U1-G1 & U2-G2 & U2-UL2 & G2-GL2 & U1-U2 & G1-G2 \\
\hline 100 & 0.0592 & 0.0467 & 0.1397 & 0.1392 & 0.4798 & 0.4664 \\
\hline 200 & 0.0463 & 0.0423 & 0.1353 & 0.1346 & 0.4111 & 0.4041 \\
\hline
\end{tabular}

\section{Packing density effects}

The effects of different packing densities are observed by comparing U2 against UL2, and G2 against GL2. The PVs of UL2 and GL2 are displayed in Figure 6.7 and the corresponding dissimilarity coefficients are listed in Table 6.6. Clearly, the dissimilarity caused by the packing density is larger than that of the particle distribution. 


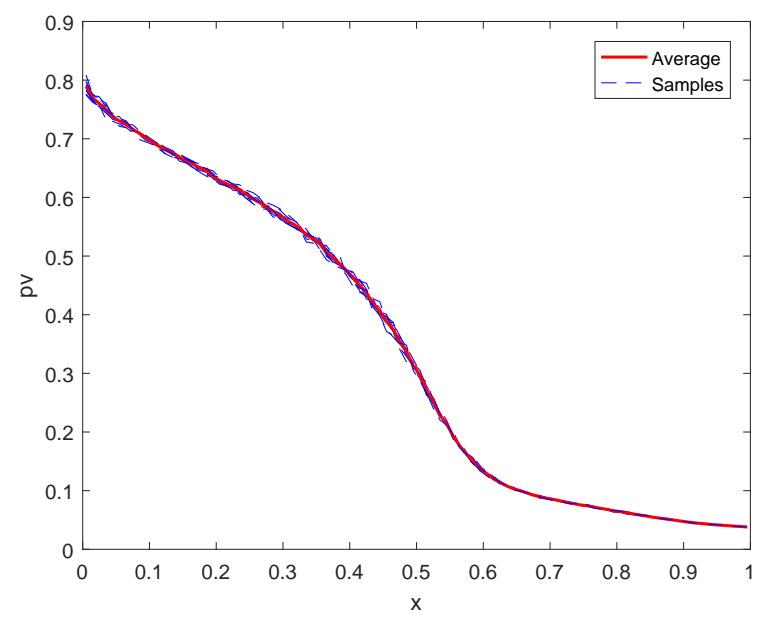

(a) $\mathrm{U} 1(100)$

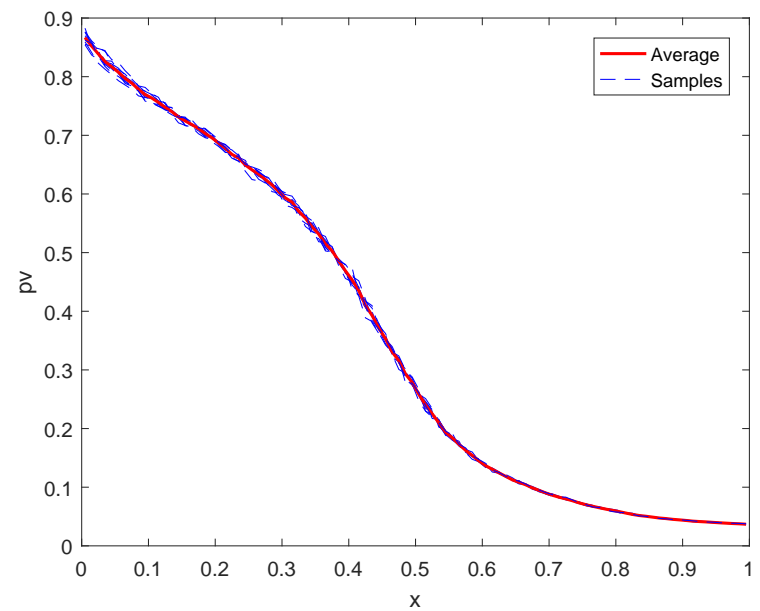

(c) G1(100)

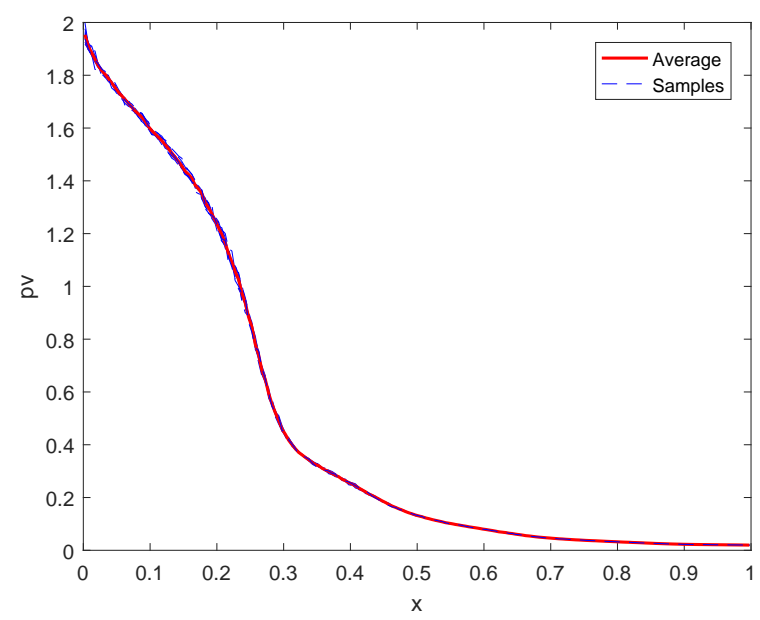

(b) $\mathrm{U} 1(200)$

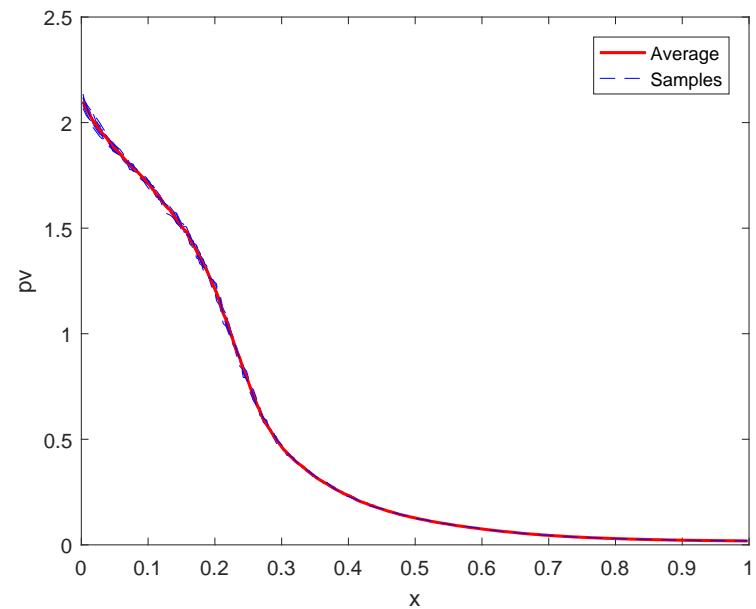

(d) G1(200)

Figure 6.5: Average principal variance functions of groups U1 and G1 for N=100, 200

\section{Particle size effects}

Finally, the effects of particle sizes with the same distribution nature (uniform or Gaussian, for instance) are investigated by comparing U1 against U2, and G1 against G2. The dissimilarity coefficients of U1-U2 and G1-G2 are also listed in Table 6.6 which are significantly larger than the others.

\section{Summary}

To visualise the effects caused by different packing properties, all DCs are plotted in Figure 6.9. It can be addressed that the randomness and particle distribution of the packings have minor influence on the packing features compared to packing density and particle size. 


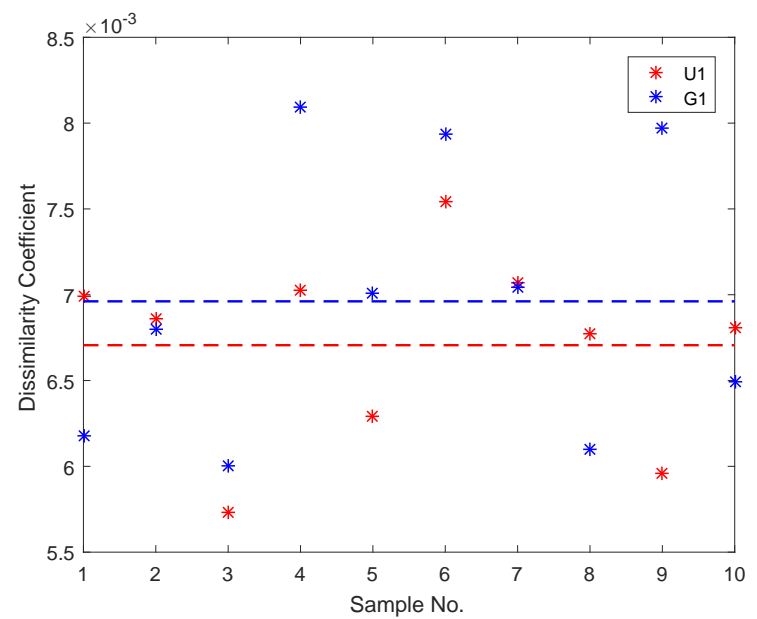

(a) $\mathrm{N}=100$

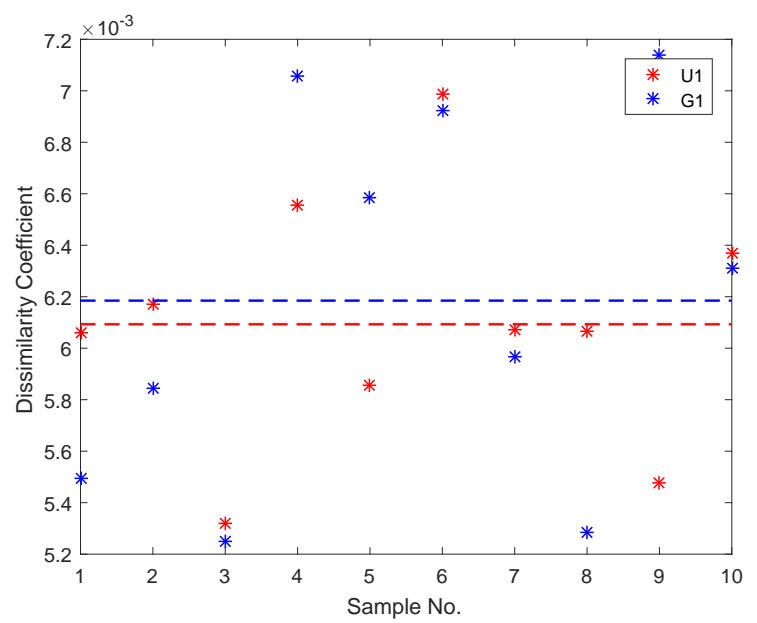

(b) $\mathrm{N}=200$

Figure 6.6: Dissimilarity coefficients of groups U1 and G1 for N=100, 200

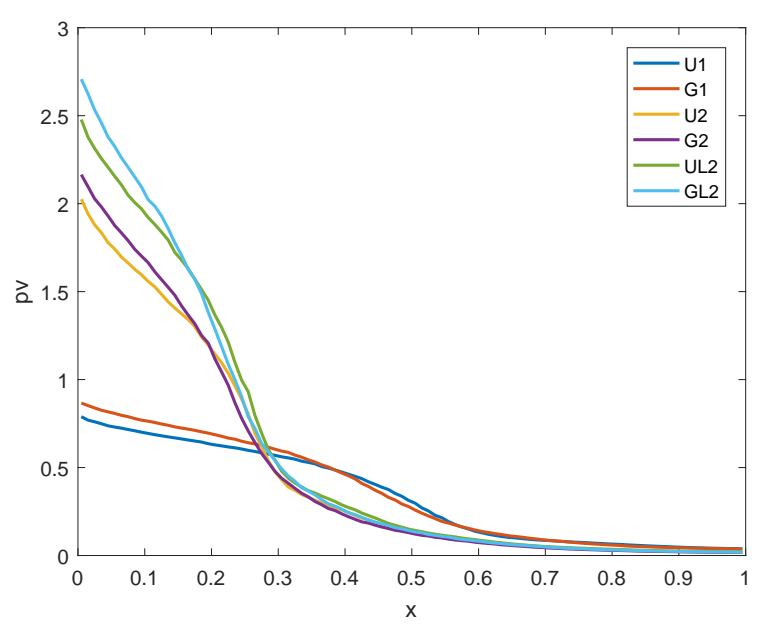

(a) $\mathrm{N}=100$

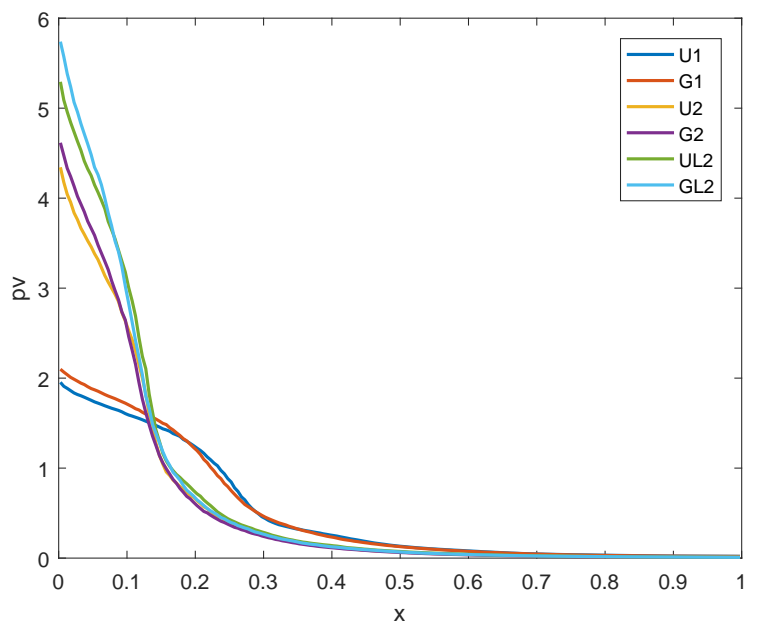

(b) $\mathrm{N}=200$

Figure 6.7: Average principal variance functions of different groups

\subsubsection{Packing uniformity and isotropy}

Besides having capability of comparing the features of two particle packings, this PCA based approach can also be employed to exploit the properties of a packing in more detail, such as its spatial uniformity and isotropy.

\section{Uniformity}

For one packing, its uniformity in space can be checked by applying a moving analysis region and obtaining the PVs at some selected locations (as shown in Figure 6.10), and then compute the DCs of these PVs by (5.25). A uniformly small DC indicates that the packing may be statistically homogeneous. Alternatively, if the volume matrix $\mathbf{V}_{h}$ of a packing within a large analysis region is obtained, by selecting smaller analysis regions with the same grid spacing $h$, 


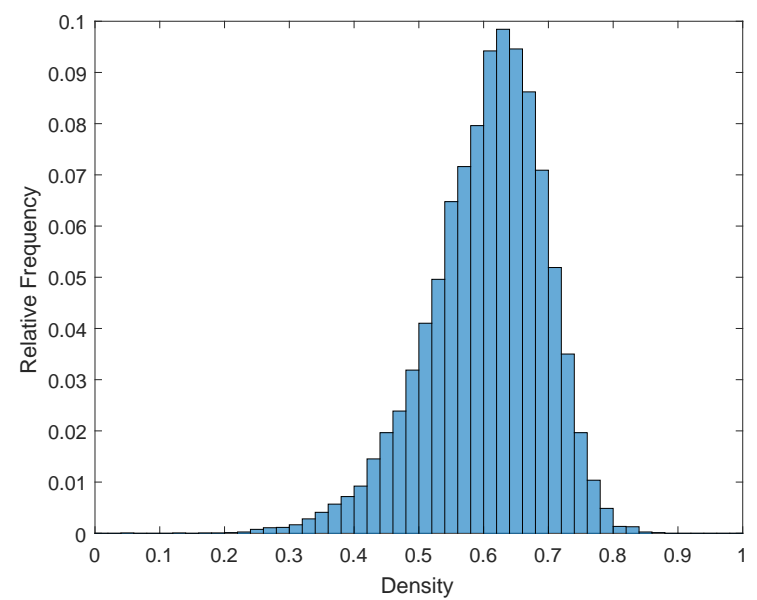

(a) $\mathrm{N}=25$

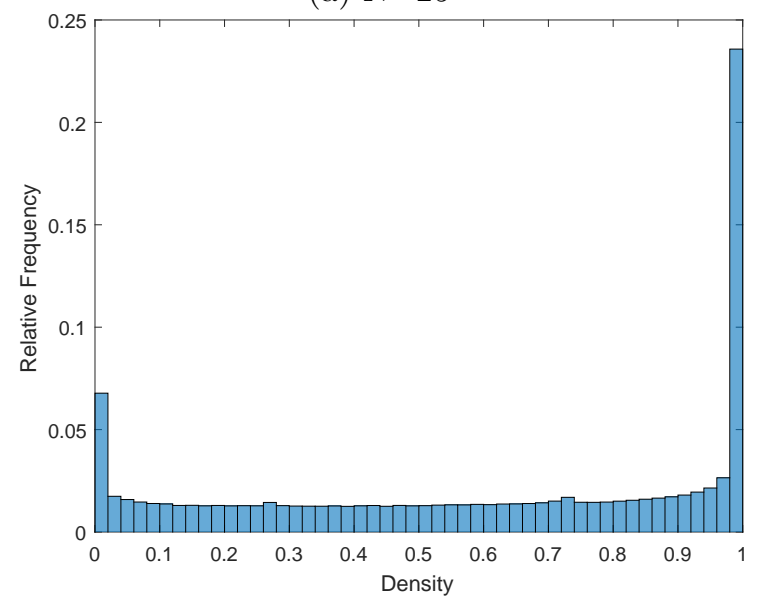

(c) $\mathrm{N}=100$

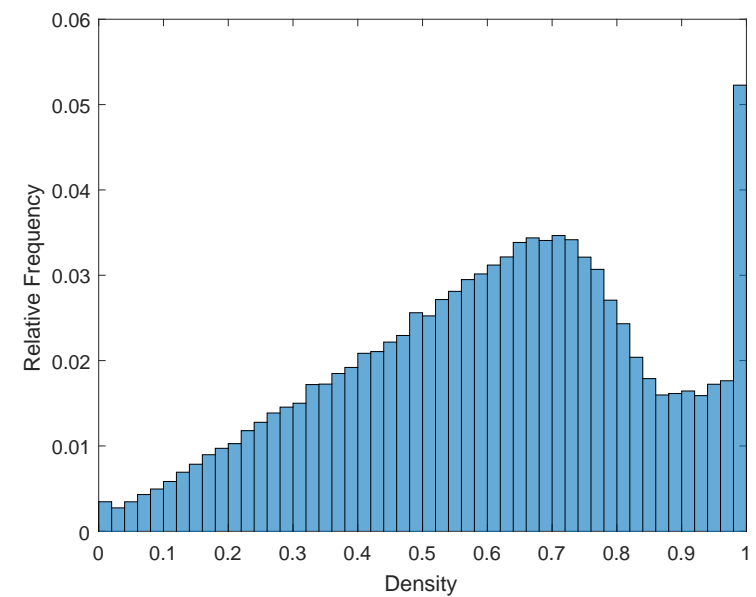

(b) $\mathrm{N}=50$

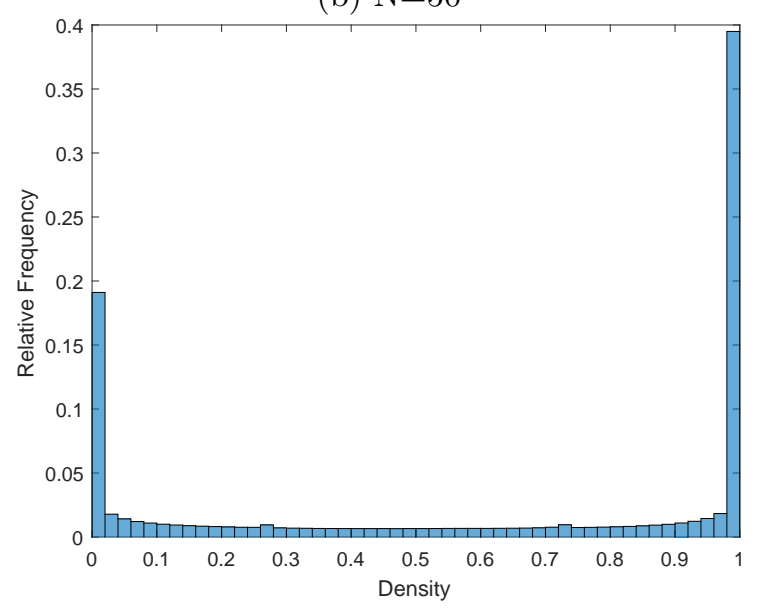

(d) $\mathrm{N}=200$

Figure 6.8: Density profiles of the U1 packing with different resolutions

or by simply selecting sub-matrices from $\mathbf{V}_{h}$, the spatial uniformity of the packing can also be investigated at a smaller scale.

To illustrate this approach, U1(200) and L(200) are used as two examples. 10 of $[100 \times 100 \times 100]$ sub-matrix blocks are randomly extracted from the two packing volume matrices and principal component analysis is applied to every sub-matrix to obtain its PVs. Figure 6.11(a) shows PVs of the sub-matrices and the averages of different packings. Clearly the PV functions of 10 sub-matrices of Set U1 are located within a narrow band around the mean value while those of Set L show notable variations. The dissimilarity coefficients of the sub-matrices against the averaged value are depicted in Figure 6.11(b). A small level of spatial in-homogeneity exists in Packing U1 while a large degree of in-homogeneity is observed in Packing L, as expected.

\section{Isotropy}

The isotropy of a packing can be examined by rotating an analysis region in three directions, and applying principal component analysis to each analysis region, as displayed in Figure 6.10(b). 


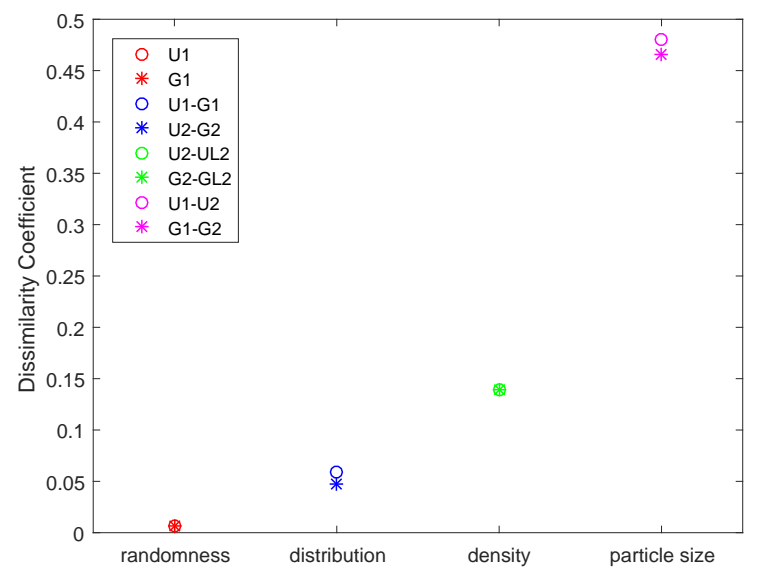

(a) $\mathrm{N}=100$

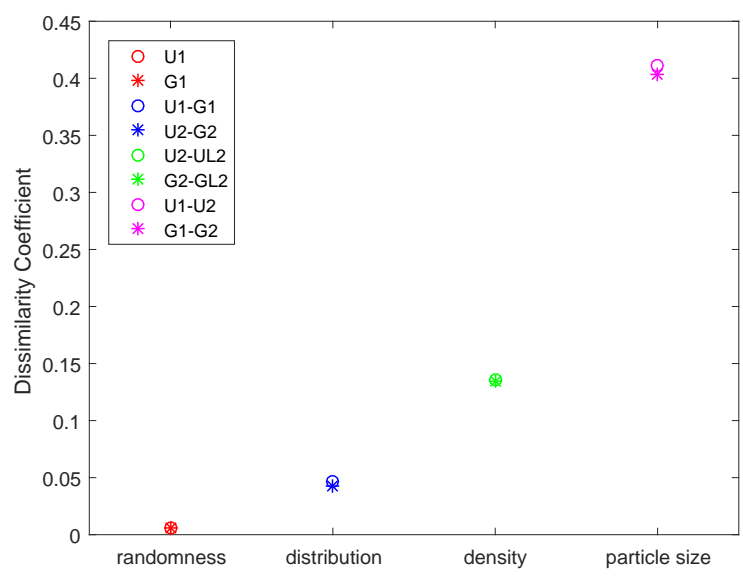

(b) $\mathrm{N}=200$

Figure 6.9: Dissimilarity coefficients of different samples and groups

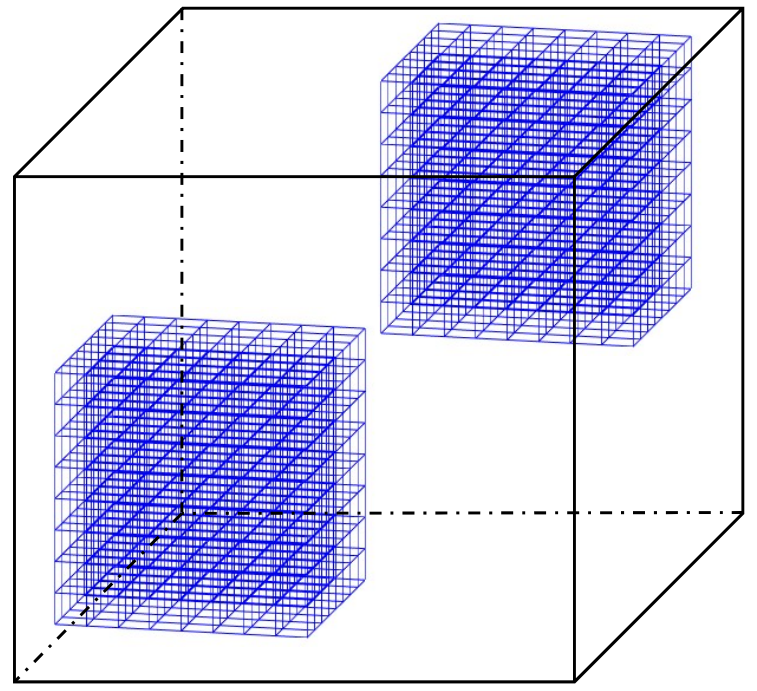

(a) Uniformity

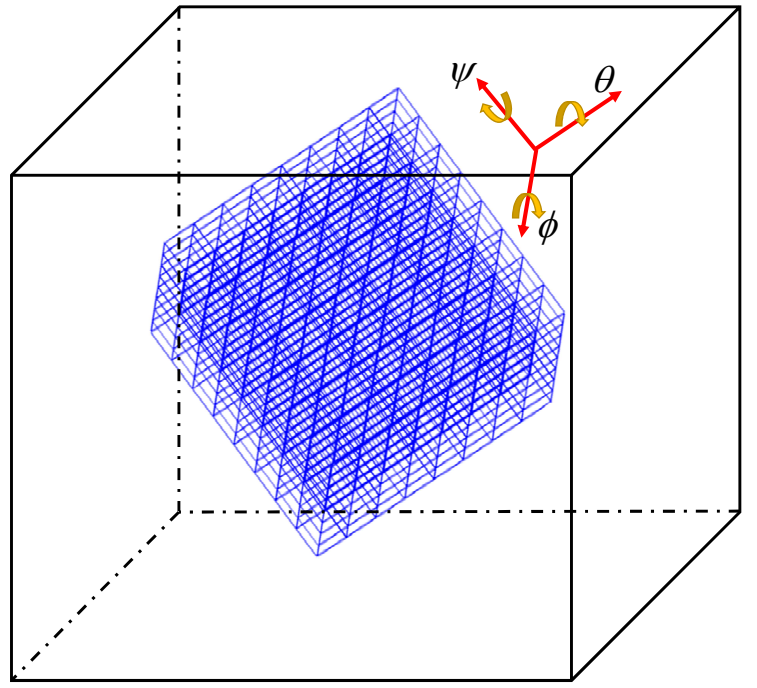

(b) Isotropy

Figure 6.10: Uniformity and isotropy checks of a packing

Then the DCs of the PVs of these rotated analysis regions against their averaged PVs will reveal if the given packing is generally isotropic or not.

For illustrative purpose, this approach is applied to Packings U1 and L. The analysis region is dimensioned as $[0.5 \times 0.5 \times 0.5]$ with the resolution $[50 \times 50 \times 50]$. Seven values of rotate angles $\theta, \phi, \psi$ are chosen from $0^{\circ}$ to $180^{\circ}$ as $\left[0^{\circ}, 30^{\circ}, 60^{\circ}, 90^{\circ}, 120^{\circ}, 150^{\circ}, 180^{\circ}\right]$. Then the total number of rotated analysis regions is 343 . Figure 6.12 shows PVs of the rotated regions and the averages of Packings U1 and G. Obviously, the PV function of Packing U1 is smaller and stabler than that of Packing L. The dissimilarity coefficients of the rotated analysis regions against the averaged value are plotted in Figure 6.13. The average DC of Packing U1 is 0.0186 but that of Packing L is 0.0443 , which indicates that a weak isotropy exists in Packing U1 while a notable an-isotropy exists in Packing L. 


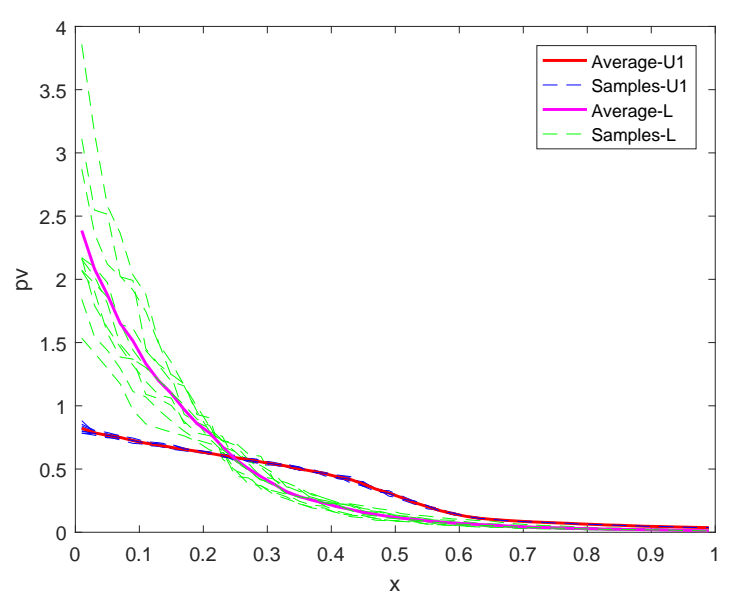

(a) Principal variances

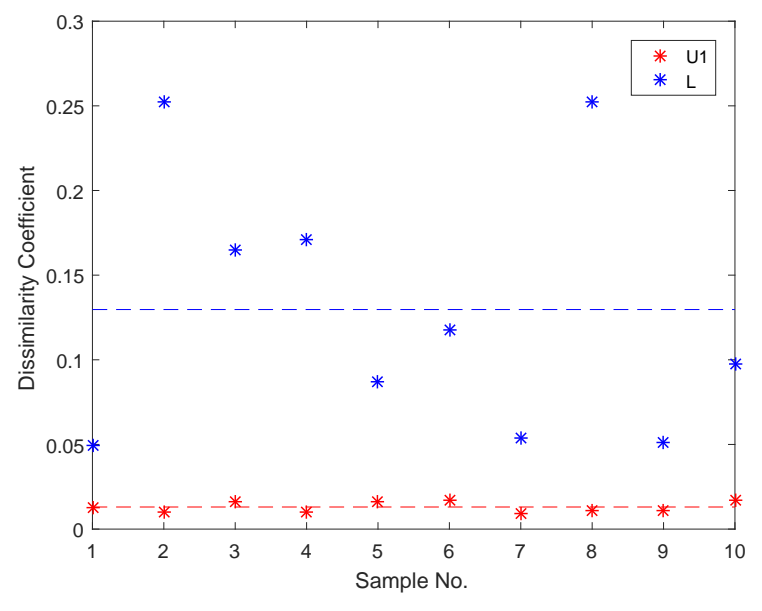

(b) Dissimilarity coefficients

Figure 6.11: Packing uniformity checkings for U1 and L

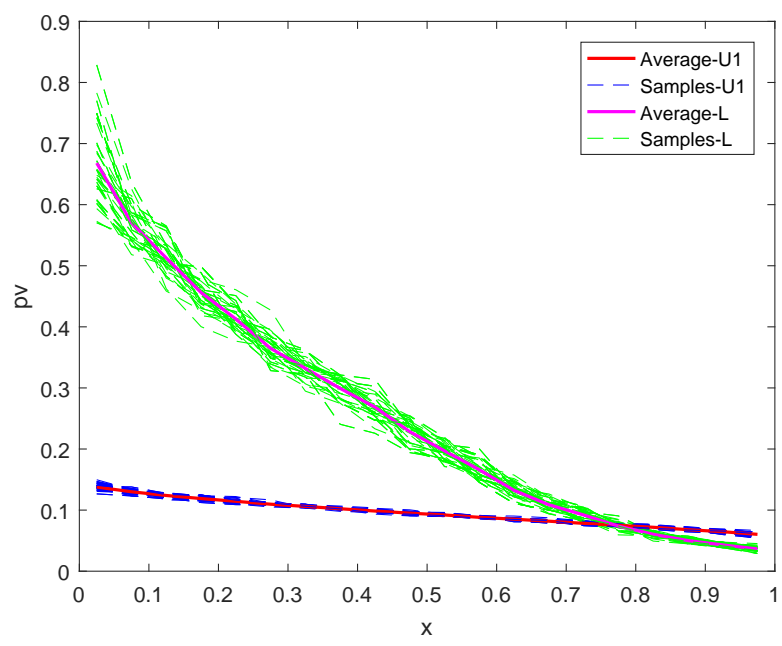

Figure 6.12: Principal variances for isotropic checking

\subsection{Packing Evolution of a Particle System Subject to Tri- axial Loading}

To illustrate how the principal component analysis can investigate the microstructure evolution of a particle packing, a triaxial compression test is conducted on the packing U1 using DEM simulation (Figure 6.14(a)). By applying the so-called servo-control algorithm, the packing is isotropically compacted to reach two isotropic confinement stress levels of $0.5 \mathrm{MPa}$ and 0.6 MPa. The linear contact model is adopted, and the numerical parameters used are as follows: particle density $\rho=2500 \mathrm{~kg} / \mathrm{m}^{3}$, normal contact stiffness $k_{n}=1.5 \times 10^{8} \mathrm{~N} / \mathrm{m}$, shear contact stiffness $k_{s}=1.5 \times 10^{8} \mathrm{~N} / \mathrm{m}$ and frictional coefficient $f=0.3$. The vertical compression, with the confinement stress remaining constant in the two lateral directions, is performed on the packing to reach the final axial strain of 0.3. Figure 6.14(b) shows the stress curves and the corresponding variations of the Dissimilarity Coefficient under the lateral compression stresses of $0.5 \mathrm{Mpa}$ and $0.6 \mathrm{Mpa}$. 35 different states are chose during the compression test and 


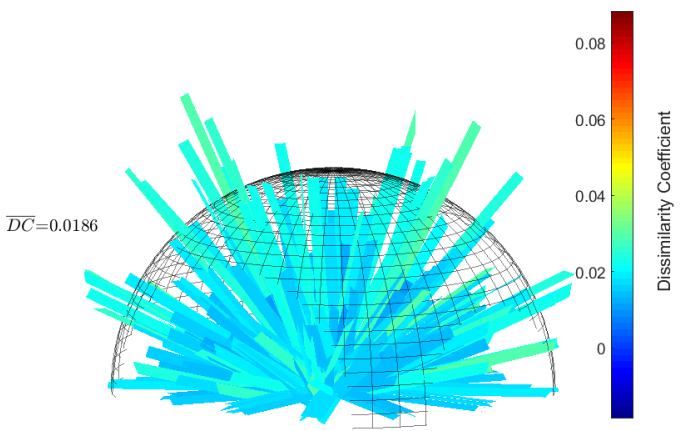

(a) Packing U1

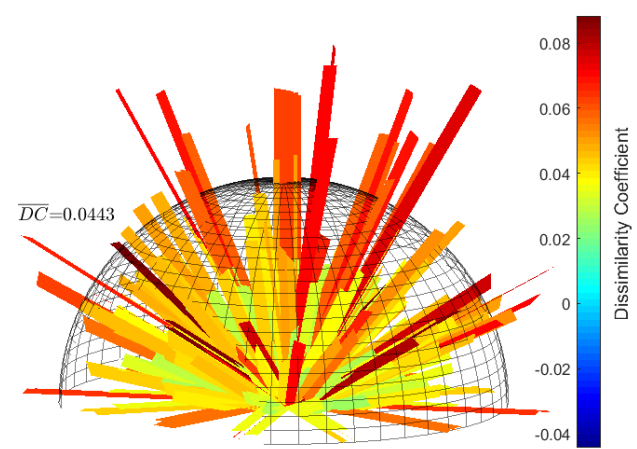

(b) Packing L

Figure 6.13: Dissimilarity coefficient for isotropic checking

the corresponding PV functions are obtained respectively. The DC values are calculated by regarding the initial packing under the isotropic stress state as the reference packing.

The stress curves exhibit the typical behaviour of granular materials. It can be observed that the DC curves have the same variation tendency as the stress curves. At the initial stage of the compression, the DC increases rapidly, indicating that an obvious configuration change occurs in the sample. The state of the sample under the peak strength shows the largest configuration difference from the initial state. Then as the sample reaches the critical state, the configuration of the packings fluctuates. It is demonstrated that the macroscopic response stress may have an internal relation with the spatial distribution of the void ratio and this relation may be revealed by the Principal component analysis. At the current stage, however, it is not easy to explain the mechanism of the effects of spatial distribution of void ratio on the bulk response of the particulate packing.

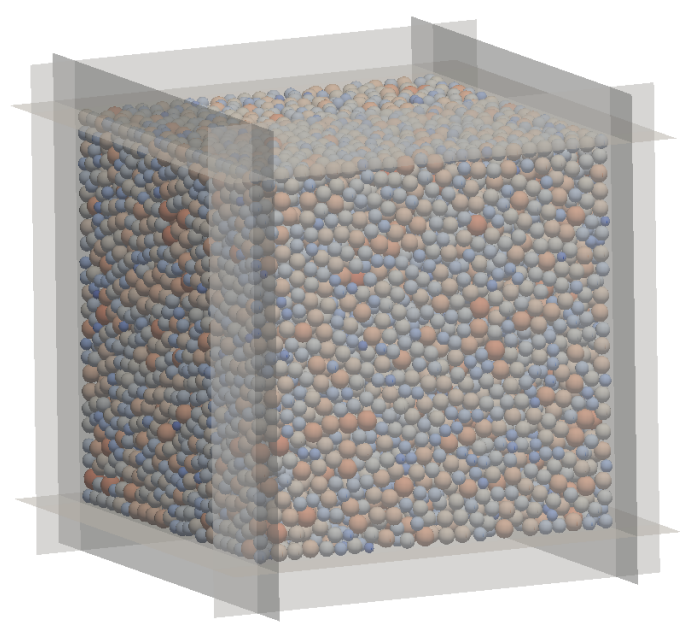

(a)

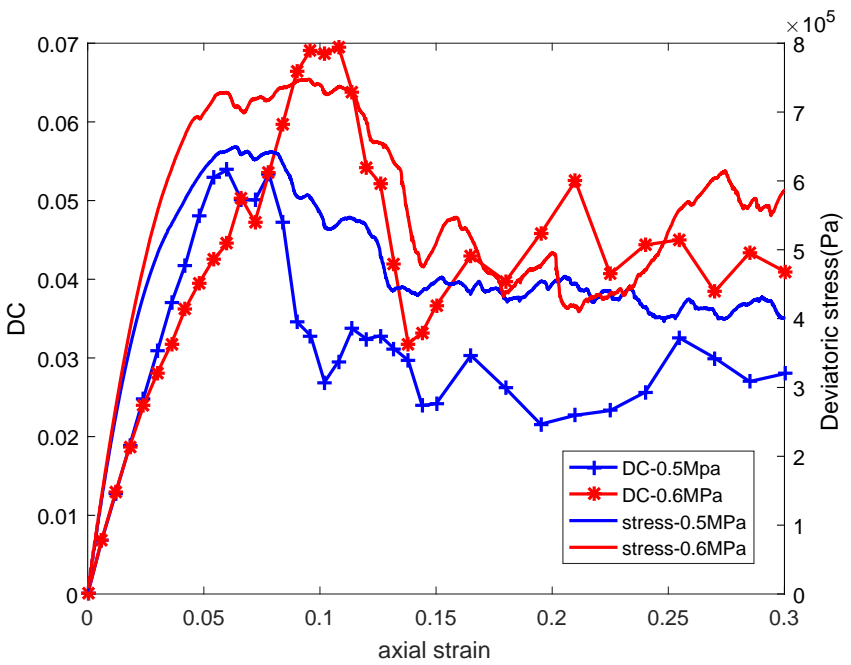

(b)

Figure 6.14: (a)Three dimensional compression (b)Deviatoric stress and Dissimilarity coefficient (DC) evolution 


\subsection{Concluding remarks}

This chapter has extended the methodology which characterises the particle packing based on Principal Component Analysis to 3D cases. Gaussian Quadrature is adopted to obtain the volume matrix of a particle packing. Then the digitalised image of the packing is obtained by converting the image matrix along the $z$-direction into a column vector of the image matrix of the packing. Both principal variance function and dissimilarity coefficient are proposed to compare different packing images.

This approach has been applied to several purposefully generated packings. Numerical investigations have shown that differences between two packings with different features can be characterised by the principal variance and dissimilarity coefficient. The values of PVs and DC can indicate different levels of effects on packing caused by configuration randomness, particle distribution, packing density and particle size. The uniformity and isotropy of a packing can also been investigated by this PCA based approach. Although only spherical particle packings are considered, other non-spherical packings can equally be treated within the proposed characterisation methodology.

It has also showed a correction between the macroscopic response and the difference of the packing configurations of a sample, while the mechanism of how microscopic structures affect the macroscopic strength of the packing using PCA is unclear at the current stage. The packing characterisation through the illustrative examples has been mainly focused on the quantitative comparison between different packings or different regions/orientations of a single packing using their principal variances or the dissimilarity coefficient. More work is being pursued to directly relate PVs and other values in the characteristic sets to packing features of a packing. 


\section{Part IV}

\section{Multiscale modelling}




\section{Chapter 7}

\section{Exact scaling laws and coarse graining methods}

\subsection{Introduction}

When the DEM simulation is performed by using larger than real sized particles, or coarse grained particles, some theoretical issues need to be addressed to understand the difference between the original system and the coarse grained system. Moreover, a systemic framework needs to be proposed to develop a reliable coarse grained system which can effectively represent the original system.

Consider a simple regular packed particle system and a coarse grained system as shown in Figure 7.1. It should be noted that the regular arrangement of the particles here is just for illustrative purposes. As can be seen, the characteristic dimension of both systems keep the same while the characteristic dimension of the discrete elements has been enlarged by a factor of 2 in the coase grained system. In the previous work of Feng [220], a parameter called the scale number $S_{n}$ is defined as the ratio of the characteristic dimension of the system to the the characteristic dimension of the particles in the system, and has been introduced to represent the difference between the original system and the coarse grained system. It is obvious that the scale number of the original system is larger than that of the coarse grained system. While how does this scale number affect the performance of the whole system and how to guarantee the two systems with a similar performance need more discussion.

This problem can be treated from two aspects. On one hand, from the particle level, the governing equation for particle motion and the interaction law involved should be scaled properly. On the other hand, from the system level, the difference caused by the configuration of the particles should be considered.

To solve this problem step by step, the exact scaled system is presented in the previous work $[220,221]$, in which the characteristic dimension of the system and the elements are scaled simultaneously (see Figure 7.1). The exact scaled model is a geometrically exact 


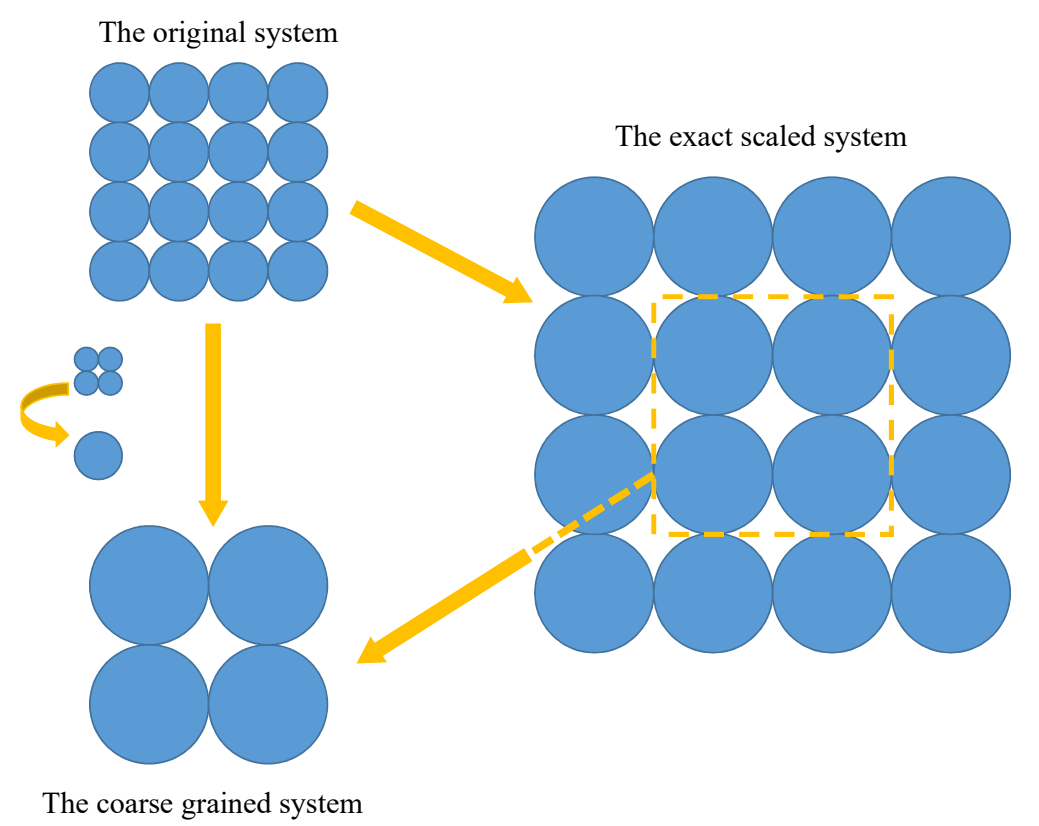

Figure 7.1: The original system, the exact scaling system and the coarse graining system

representation of the original problem, i.e. both models have the same particle number and particle packing configuration while this condition cannot be kept between the coarse grained system and the original system. The coarse grained system could be seen as a part of the exact scaling system. The particle sizes and the domains in the original and exact scaled models are different only by a constant (spatial) scale factor $h$. In what follows, the overbar ${ }^{-}$ will be used to denote a quantity associated with the exact scaled system.

Let $R$ and $\bar{R}$ be the radii of an arbitrary particle in both the original and exact scaled models respectively, and $D$ and $\bar{D}$ denote the characteristic lengths of the domains in both models. Then

$$
\bar{R}=h R ; \bar{D}=h D ; \bar{S}_{n}=S_{n}
$$

In this situation, the scale number $S_{n}$ remains the same for both models. A set of scaling laws has been proposed to assure that the exact scaled system can exactly represent the original system. The scaling conditions for the exact scaled system is briefly introduced in Section 7.2. The existing coarse graining techniques developed by other researchers are all intended to keep the similarity between the original system and the scaled system on the particle level. Section 7.3 aims to revisit some of existing coarse grain technologies and evaluate them within our generic theoretical framework based on the exact scaled system.

Then the next step is to tackle this problem on the system level. The equivalent dynamical properties of the original system and the exact scaled system can be guaranteed by applying the scaling conditions on the exact scaled system. However, there will be inevitable difference between the coarse grained system and the original system caused by the different packing configurations. It could also be explained that the coarse grained system is only a part of the exact scaled system (see Figure 7.1) which will show some differences compared to the whole 
system.

\subsection{Scaling conditions for exact scaled system}

A complete set of scaling conditions under which the exact scaled system can exactly reproduce the behaviour of the original system has been established in the previous work [220]. The conditions are derived from the governing equations in a straightforward manner, based on simple unit conversions of all the physical quantities involved in both physical and scaled models.

The mechanical motion of the particle system is fully governed by Newton's second law. The governing equations for an arbitrary particle can be generally expressed as

$$
m \ddot{\mathbf{u}}(t)+\mathbf{F}_{\mathrm{d}}(t)+\mathbf{F}_{\text {int }}(t)=\mathbf{F}_{\text {ext }}(t)
$$

where $m$ is the mass of the particle, $\mathbf{F}_{\mathrm{d}}$ is the damping force accounting for any energy dissipation in the system, $\mathbf{F}_{\text {int }}$ is the resultant of all the interaction forces from other particles or other phases defined by the interaction laws, $\mathbf{F}_{\text {ext }}$ is the resultant external force applied if any, and $\ddot{\mathbf{u}}$ is the acceleration.

The governing equations for the particle in the exact scaled system could be written as

$$
\bar{m} \overline{\mathbf{u}}(t)+\overline{\mathbf{F}}_{\mathrm{d}}(t)+\overline{\mathbf{F}}_{\text {int }}(t)=\overline{\mathbf{F}}_{\text {ext }}(t)
$$

To ensure the results obtained in the exact scaled system can be exactly scaled back to the results for the original system, the above two equations should be mathematically equivalent, or simply differ only by a constant factor. Unlike the classic dimensional analysis [222, 223] or some earlier work on the development of scaling laws for particle systems [224], Feng's previous work takes a relatively simple approach which ensures that all the corresponding forces involved in both systems are proportional:

$$
\frac{\bar{m} \overline{\ddot{u}}}{m \ddot{u}}=\frac{\bar{F}_{\mathrm{d}}}{F_{\mathrm{d}}}=\frac{\bar{F}_{\mathrm{int}}}{F_{\mathrm{int}}}=\frac{\bar{F}_{\mathrm{ext}}}{F_{\mathrm{ext}}}=\lambda
$$

A set of scaling laws are established on this basis by a more straightforward approach which aims to determine the scale factors for all the individual physical quantities involved in the particulate system. Let $q$ be an arbitrary physical quantity in the system, and $\lambda_{q}$ is the scale factor for $q$

$$
\bar{q}=\lambda_{q} q
$$

Physical quantities are interdependent and can be derived from a few basic quantities. The three basic quantities in our work are Length $[L]$, Mass density $[\rho]$ and Time $[T]$ and the 
Table 7.1: Scale factors for some physical quantities in the exact scaling system

\begin{tabular}{|c|c|c|c|}
\hline Quantity & Symbol & $\begin{array}{c}\text { Dimension } \\
\lambda\end{array}$ & Scale factor \\
\hline Length & $L$ & {$[L]$} & $h$ \\
Time & $t$ & {$[T]$} & $h$ \\
Density & $\rho$ & {$[\rho]$} & 1 \\
Force & $F$ & {$[\rho][L]^{4}[T]^{-2}$} & $h^{2}$ \\
Strain & $\epsilon$ & {$[1]$} & 1 \\
Stress & $\sigma$ & {$[\rho][L]^{2}[T]^{-2}$} & 1 \\
Kinetic energy density & $e_{k}$ & {$[\rho][L]^{2}[T]^{-2}$} & 1 \\
Strain energy density & $e_{s}$ & {$[\rho][L]^{2}[T]^{-2}$} & 1 \\
\hline
\end{tabular}

corresponding scale factors for these three quantities are specifically chosen as

$$
\lambda_{L}=h ; \lambda_{T}=h ; \lambda_{\rho}=1
$$

i.e. the scale factors for length and time are the same as the spatial scale factor.

After the scale factors for the basic quantities between the original and exact scaled systems are defined, the scale factors for other quantities can be derived easily as shown in Table 7.1 (see [220] for details). These choices of the scale factor for the basic quantities lead to a desirable result. The scale factors for particle stress, strain, kinetic energy density, strain energy density are unity, which ensures the similarity between the original system and the exact scaled system.

Applying these scaling laws into the DEM simulation could be accomplished by two possible ways. One desirable way is to apply the required scale factor to all the quantities involved. Another way named partial scaling approach has been explained elaborately in [220]. Some existing coarse graining approaches of other researchers could be treated as tackling the problem by the latter way.

As the same time-stepping integration scheme will be adopted for the original system and the exact scaled system, it is not difficult to deduce that the scale factor for the time step associated with the scheme should be $\lambda_{\Delta t}=\lambda_{T}=h$, which implies that the same number of time steps are required in the exact scaled model as in the original model. In other words, the same computational costs will be involved in solving both original and exact scaled models, and thus the exact scaling offers no computational cost savings.

\subsection{Coarse graining methods revisited}

To reduce computational costs for a large scale problem, some non-exact scaling approaches, named as coarse graining methods, have been proposed which can be classified into three categories. The first method develops a specific model in which a large-sized particle is used 
to represent a group of original particles and then a series of relationships between the original system and the coarse grained system are derived. The particle-fluid system is the main study object of this method. The second method applies dimensional analysis to guarantee the equivalent properties of the two systems based on the motion equation. The third method focus on the interaction law directly.

Although the exact scaling approach cannot improve the calculation efficiency, the scaling laws proposed can serve as the basic principles to guarantee the similar behaviour of particles with different sizes, which is also the objective of other coarse graining approaches. Therefore, it is reasonable to incorporate those coarse graining approaches into the theoretical framework based on the exact scaling system considering its simplicity and general applicability. This feature can be seen more clearly when compared with those existing coarse graining approaches.

\subsubsection{Representative model}

Coarse graining is based on an intuitive thought that a group of small particles could be represented by a large particle to reduce the problem size. The coarse grained model in Figure 7.1 is obtained by replacing four small particles by a large particle. The approach based on this concept has been the most commonly used method for coarse graining. The most important feature of this approach is that the particle-particle and fluid-particle interaction forces of the representative particles are calculated using the physical properties of the original particles. Only the detection of contacts or collisions between particles is performed using the diameter of the representative particles. Such forces need to be scaled properly to ensure the similarity between the original system and the coarse grained system. The governing equation of this approach is similar to Equation (7.2). Unlike the systemic way that scale factors are obtained in the dimensional analysis, the scale factors here are derived based on a number of assumptions. Here, two models in this category which are cited frequently are investigated in detail.

\section{1) Similar particle assembly (SPA)}

The first one is the similar particle assembly (SPA) model developed by Kuwagi [139] as shown in Figure 7.2. The original system consists of a bed of particles. These particles are grouped together in which their size, density, and chemical composition are similar. Their velocity and direction of motion are also similar. The representative system contains a set of representative particles that are assumed to occupy an equal space size with the size $h$ times larger than the original particles. A similar particle assembly model is established because the spatial arrangement of the original system and that of the representative system is analogous. Use $i$ and $i^{\prime}$ to denote particle indices in the original system and in the representative system respectively below. 


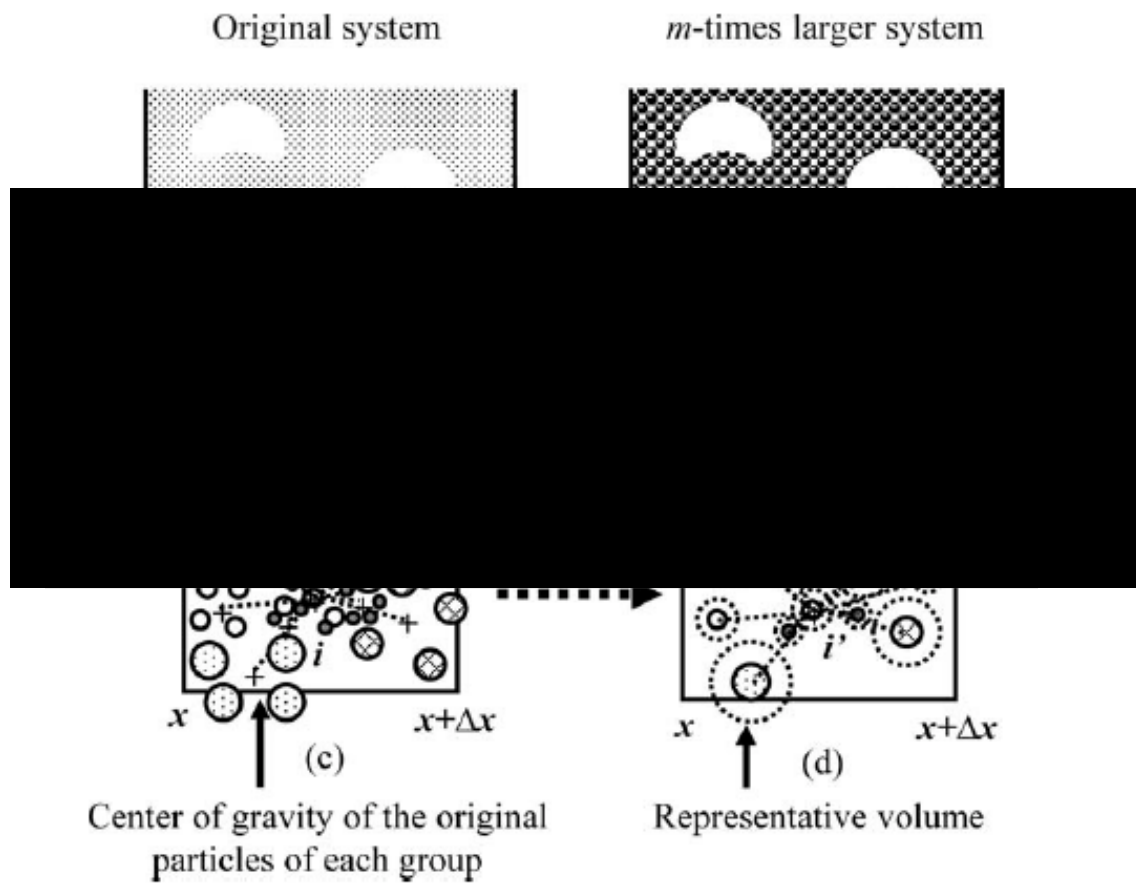

Figure 7.2: Diagram of the scaling law for the similar particle assembly (SPA) model [141]

The governing equation for particle motion in the original system takes the following form

$$
\rho_{\mathrm{p}(i)}\left(\frac{\pi}{6} d_{\mathrm{p}(i)}^{3}\right) \frac{d \mathbf{v}(i)}{d t}=\sum_{i \neq j} \mathbf{F}_{\mathrm{p}(i j)}+\mathbf{F}_{\mathrm{fp}(i)}+\rho_{\mathrm{p}(i)}\left(\frac{\pi}{6} d_{\mathrm{p}(i)}^{3}\right) \mathbf{g}+\mathbf{F}_{\mathrm{coh}}
$$

where $\rho_{\mathrm{p}(i)}$ is the particle density, $d_{\mathrm{p}(i)}$ is the particle diameter, $i, j$ are the particle indices, $\mathbf{F}_{\mathrm{p}(i j)}$ is the contact force between two contacting particles $i$ and $j, \mathbf{F}_{\mathrm{fp}(i)}$ is the fluid-particle interaction force, $\mathbf{F}_{\mathrm{coh}}$ is the liquid bridge force. It is easy to figure out that this equation is just another equivalent expression of Equation (7.2).

The particle sizes in the representative system and the original system follow the scaling relationship

$$
d_{\mathrm{p}\left(i^{\prime}\right)}=h d_{\mathrm{p}(i)}
$$

The corresponding equation of (7.7) for the coarse grained (representative) system becomes

$$
h^{3} \rho_{\mathrm{p}\left(i^{\prime}\right)}\left(\frac{\pi}{6} d_{\mathrm{p}(i)}^{3}\right) \frac{d \mathbf{v}\left(i^{\prime}\right)}{d t}=\sum_{i^{\prime} \neq j^{\prime}} \overline{\mathbf{F}}_{\mathrm{p}\left(i^{\prime} j^{\prime}\right)}+\overline{\mathbf{F}}_{\mathrm{fp}\left(i^{\prime}\right)}+h^{3} \rho_{\mathrm{p}\left(i^{\prime}\right)}\left(\frac{\pi}{6} d_{\mathrm{p}(i)}^{3}\right) \mathbf{g}+\overline{\mathbf{F}}_{\mathrm{coh}}
$$

The scaling law for the terms in this equation is proposed subjectively without theoretical analysis. It is believed that the particle diameter has a major impact on the hydrodynamic behaviour of the particle, so the scaling law can be applied directly to particle-particle and particle-fluid interaction forces, as shown below 


$$
\sum_{i^{\prime} \neq j^{\prime}} \overline{\mathbf{F}}_{\mathrm{p}\left(i^{\prime} j^{\prime}\right)}+\overline{\mathbf{F}}_{\mathrm{fp}\left(i^{\prime}\right)}+\overline{\mathbf{F}}_{\mathrm{coh}}=h^{3}\left(\sum_{i \neq j} \mathbf{F}_{\mathrm{p}(i j)}+\mathbf{F}_{\mathrm{fp}(i)}+\mathbf{F}_{\mathrm{coh}}\right)
$$

The author claimed that if Equation (7.10) is satisfied and the density of the space represented by a representative particle is the same as the original particle, then the velocity congruity can be established by further comparing Equations (7.7) and (7.9) as

$$
\mathbf{v}_{\left(i^{\prime}\right)}=\mathbf{v}_{(i)}
$$

The advantage of this model is obvious that in the coarse graining system the physical properties of the original particles can be used directly. The diameter of the representative particle is only used when perform the detection of contacts or collisions between particles. To put it simply, the author just applies a scale factor of $h^{3}$ to all the terms in the governing equation, but there is a contradiction in this SPA model.

From Equation (7.11), the author draws the conclusion that particles in the coarse grained system has a motion that is similar to the that of the corresponding original particles. If based on the exact scaling system, the scale factor of length for the SPA model is $h$, as the scale factor of velocity is 1 , thus the scale factor of time is also $h$, and the same for the scale factor of time step. If we compare the left hand sides of Equations (7.7) and (7.9), no scaling is applied to the term of $d t$ which is a contradiction between the SPA model and the conclusion based on the exact scaling laws. If a scale factor $h$ is applied to $d t$, then the total scale factor of the left hand side of Equation (7.10) is $h^{2}$, therefore the force scale factor for the SPA should be changed from $h^{3}$ to $h^{2}$.

\section{2) Coarse grain model}

To simulate a pneumatic conveying, Sakai $[140,225]$ developed the coarse grain model based on the SPA approach with more details. As described in Figure 7.3, there are $h^{3}$ original particles in the (3D) coarse grain particle whose size is $h$ times larger than the original particle. As shown in Figure 7.3(a), the translational motion of the coarse grain particle is assumed to be the same as that of the group of original particles. Therefore, the velocity and displacement of the coarse grain particle is assumed to be the average of those of the original particles. As far as the rotational motion is concerned, the original particles existing in the coarse grain particle each are assumed to rotate around their own center of mass with the same angular velocity, as illustrated in Figure 7.3(b). The contact force acting on the coarse grain particle is estimated under the assumption that the kinetic energy of the coarse grain particle agrees with that of the original particles. The drag force and external force are modeled by balancing the coarse grain particle with the group of the original particles. For the cohesive particles, the van der Waals force is modelled based on the assumption that the potential energy of the coarse grain particle is the same as the original particles. Consequently, the following 


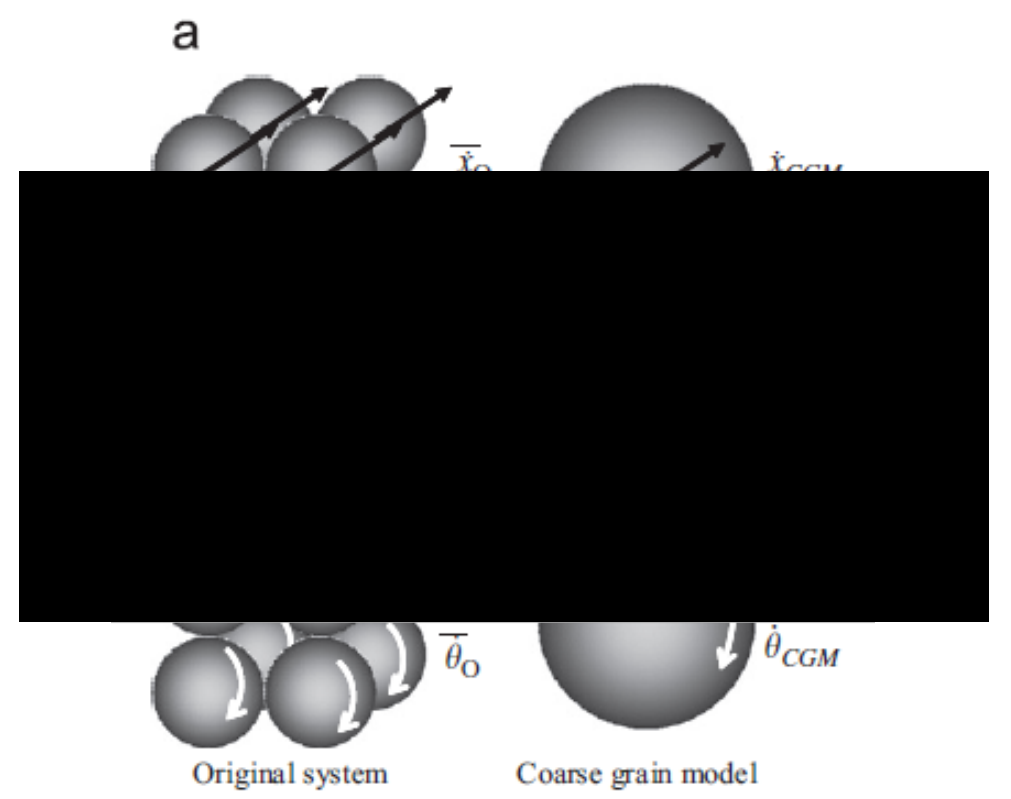

Figure 7.3: Coarse grain model (a)Translation; (b)Rotation [140]

relationship is obtained between the coarse grain particles and the original particles

$$
\begin{aligned}
m_{\mathrm{CGM}} \dot{\mathbf{v}}_{\mathrm{CGM}} & =\mathbf{F}_{f_{\mathrm{CGM}}}-V_{\mathrm{CGM}} \nabla p+\sum \mathbf{F}_{C_{\mathrm{CGM}}}+\mathbf{F}_{g_{\mathrm{CGM}}}+\mathbf{F}_{v w_{\mathrm{CGM}}} \\
& =h^{3} \mathbf{F}_{f_{\mathrm{O}}}-h^{3} V_{\mathrm{O}} \nabla p+h^{3} \sum \mathbf{F}_{C_{\mathrm{O}}}+h^{3} \mathbf{F}_{g_{\mathrm{O}}}+h^{2} \mathbf{F}_{v w_{\mathrm{O}}}
\end{aligned}
$$

where $m, \mathbf{v}, h, \mathbf{F}_{f}, V, p, \mathbf{F}_{C}, \mathbf{F}_{g}$ and $\mathbf{F}_{v w}$ indicate mass, velocity, scale factor, drag force, particle volume, pressure, contact force, gravitational force and van der Waals force respectively. The subscripts of CGM and O refer to coarse grain particles and original particles respectively.

According to the author, the scale factor $h^{3}$ for the contact force is obtained under the assumption that when a binary collision in coarse grain particles occurs, the binary collisions due to all the original particles (i.e. $h^{3}$ binary collisions) are assumed to occur simultaneously. While based on the exact scaling laws, to ensure that the scale factors for particle stress, strain, kinetic energy density, strain energy density are unity, the scale factor for force should be $h^{2}$ rather than $h^{3}$. The contact forces caused by the binary collisions cannot be added up directly because contact forces of the interior particles counteracts with each other and are cancelled out. The resultant force for a group of original particles is only the sum of the forces provided by the particles on the exterior boundary with other groups of particles. It means that the force of the coarse grain particle is more like an area integral rather than a volume integral.

The scale factor of the van der Waals force is $h^{2}$ which satisfies the exact scaling laws. It is derived by the assumption that the potential energy of the coarse grain particle is the same as that of the original particles. The relationship of the potential energy between the two systems is given by 


$$
\int \mathbf{F}_{i \mathrm{pCGM}}\left(l_{\mathrm{CGM}}\right) d l_{\mathrm{CGM}}=h^{3} \int \overline{\mathbf{F}}_{i \mathrm{pO}}\left(l_{\mathrm{O}}\right) d l_{\mathrm{O}}
$$

where $l$ indicates the inter-surface distance.

The relation between the inter-surface distance of the coarse-grain particles and the original particles is expressed as

$$
l_{O}=\frac{l_{C G M}}{h}
$$

From the above two equations, a long-range force acting on the coarse grain particle can be generally expressed as

$$
\mathbf{F}_{i \mathrm{pCGM}}\left(l_{\mathrm{CGM}}\right)=h^{2} \overline{\mathbf{F}}_{i \mathrm{pO}}\left(l_{\mathrm{O}}\right)=h^{2} \overline{\mathbf{F}}_{i \mathrm{pO}}\left(\frac{l_{\mathrm{CGM}}}{h}\right)
$$

Consequently, the long-range van der Waals force acting on the coarse-grain particles is given as

$$
\mathbf{F}_{\mathrm{vdwCGM}}=h^{2} \overline{\mathbf{F}}_{\mathrm{vdwO}}
$$

The scale factors for different types of force are different in this coarse grain model because different assumptions are used to derive the scale factors. The assumption which leads to the scale factor $h^{2}$ is from the point of energy equivalence between the two systems, a fundamental physical quantity that should be kept similar. With this consideration, the scaling laws for the drag force and other external forces are also inappropriate.

Another problem in this particular coarse grain model is the time step $\Delta t$. We can find the following relation in $[140]$

$$
\Delta t<2 \pi \sqrt{m_{\mathrm{CGM}} / k_{\mathrm{CGM}}}=2 \pi \sqrt{h^{3} m_{\mathrm{O}} / h^{3} k_{\mathrm{O}}}=2 \pi \sqrt{m_{\mathrm{O}} / k_{\mathrm{O}}}
$$

This relation is not correct since all the original physical properties are used in the coarse grain model which means that $k_{\mathrm{CGM}}$ is equal to $k_{\mathrm{O}}$ not $h^{3} k_{\mathrm{O}}$. Then the relationship of the time step in the two systems can be written as

$$
\Delta t_{\mathrm{CGM}}=h^{3 / 2} \Delta t_{\mathrm{O}}
$$

From this relation, we know that the coarse graining method improves the computational efficiency by both reducing the number of particles and enlarging the time step.

In conclusion, when all the physical properties of the original particles are used in the coarse grain model, every force term in the equation of motion should be scaled by $h^{2}$. 


\subsubsection{Dimensionless analysis}

This approach could be regarded as the extension of the classic dimensional analysis [222, 223] to the DEM simulation. Firstly, the governing equation of the particulate system is written in dimensionless form. A set of non-dimensional quantities are sought from which a set of scaling laws can be identified. The detail of the dimensionless analysis can be found in the paper of Pöschel [224] which presents the method to scale down experiments to lab-size. The procedure is shown below along with the comparison with the exact scaling approach.

Consider the equation of motion below

$$
\begin{aligned}
\frac{d^{2} \delta}{d t^{2}}+\frac{B}{m \sqrt{R}}\left(\delta^{3 / 2}+\frac{3}{2} A \sqrt{\delta} \frac{d \delta}{d t}\right) & =0 \\
\left.\delta\right|_{t=0} & =0 \\
\left.\frac{d \delta}{d t}\right|_{t=0} & =v_{0}
\end{aligned}
$$

which is an equivalent form of Equation (7.2) for a contact pair. The interaction force is given by Hertz's law shown in the second term on the left side where $B$ is the elastic constant

$$
B=\frac{2 E}{3\left(1-\nu^{2}\right)}
$$

The damping force (refer to [138]) is shown in the third term where $A$ is the dissipative material constant as a function of the viscous constants $\eta_{1,2}$, the Young modulus $E$ and the Poisson ratio $\nu$.

$$
A=\frac{1}{3} \frac{\left(3 \eta_{2}-\eta_{1}\right)^{2}}{3 \eta_{2}+2 \eta_{1}} \frac{(1-\nu)(1-2 \nu)}{E \nu^{2}}
$$

To write down the above equation of motion in a dimensionless form, a characteristic length and a characteristic time are needed. The maximal compression $\delta_{0}$ is chosen as the characteristic length. It can be found by equating the kinetic energy of the impact with the elastic energy at the instant of the maximal compression

$$
m \frac{v_{0}^{2}}{2}=m \frac{B}{m \sqrt{R}} \frac{2}{5} \delta_{0}^{5 / 2}
$$

which yields

$$
\delta_{0}=\left(\frac{5}{4} \frac{m \sqrt{R}}{B}\right)^{2 / 5} v_{0}^{4 / 5}
$$

The characteristic time is defined as the time in which the particles move the distance of the characteristic length just before the collision starts

$$
t_{0}=\frac{\delta_{0}}{v_{0}}
$$


the rescaled length, time, velocity, and acceleration are expressed as

$$
\begin{gathered}
\hat{\delta}=\frac{\delta}{\delta_{0}} \\
\hat{t}=\frac{t}{t_{0}}=\frac{t v_{0}}{\delta_{0}} \\
\frac{d \hat{\delta}}{d \hat{t}}=\frac{d \hat{\delta}}{d \delta} \frac{d \delta}{d t} \frac{d t}{d \hat{t}}=\frac{1}{v_{0}} \frac{d \delta}{d t} \\
\frac{d^{2} \hat{\delta}}{d \hat{t}^{2}}=\frac{d\left(\frac{1}{v_{0}} \frac{d \delta}{d t}\right)}{d t} \frac{d t}{d \hat{t}}=\frac{\delta_{0}}{v_{0}^{2}} \frac{d^{2} \delta}{d t^{2}}
\end{gathered}
$$

So, the equation of motion can be written in the dimensionless form below

$$
\begin{aligned}
\frac{d^{2} \hat{\delta}}{d \hat{t}^{2}}+\frac{5}{4} \hat{\delta}^{3 / 2}+\frac{3}{2}\left(\frac{5}{4}\right)^{3 / 5} A\left(\frac{B}{m \sqrt{R}}\right)^{2 / 5} v_{0}^{1 / 5} \sqrt{\hat{\delta}} \frac{d \hat{\delta}}{d \hat{t}} & =0 \\
\left.\hat{\delta}\right|_{\hat{t}=0} & =0 \\
\left.\frac{d \hat{\delta}}{d \hat{t}}\right|_{\hat{t}=0} & =1
\end{aligned}
$$

To make the two systems behave identically, the value of the elastic constant $B$ and dissipative constant $A$ should be conserved by scaling the material properties involved. After conducting a complicated dimension analysis, the author derives that the scale factor of elastic constant is $h$ and that of the dissipative constant is $\sqrt{h}$ when the scale factors of length and time are $h$ and $\sqrt{h}$ respectively.

The above procedure can be incorporated into the exact scaling system. The three basic quantities are also length, time and density. If we set $\delta_{0}=h$ then $t_{0}=\sqrt{h}$, it is easy to derive the scale factors of the elastic constant and the dissipative constant based on the exact scaling system. The dimension of the elastic constant $B$ is $[\rho][L]^{2}[T]^{-2}$, therefore the scale factor should be $h$. Similarly, the dimension of the dissipative constant $A$ is $[\rho][L]^{2}[T]^{-1} /[\rho][L]^{2}[T]^{-2}$, thus the scale factor should be $\sqrt{h}$.

In the work of Bierwisch [226], such dimensional analysis has been adopted and three dimensionless numbers are obtained as

$$
\Pi_{1}=\frac{w}{R E}, \Pi_{2}=\frac{\gamma_{n}}{R \sqrt{\rho E}}, \Pi_{3}=\frac{\kappa_{t}}{R E}
$$

where $w$ is the surface energy density, $\gamma_{n}$ is the empirical damping parameter and $\kappa_{t}$ is the tangential spring constant. Thus, the scaling laws for the material properties involved are

$$
w \propto R, \gamma_{n} \propto R, \kappa_{t} \propto R
$$

There is a slight difference on the choice of the basic quantities when using our scaling system to explain the above approach. The three basic quantities in their work are length, density 
and Young's modulus with the three scale factors $h, 1$ and 1 . The scale factor for Young's modulus is decided to ensure that the energy density dissipation rates are unaffected by coarse graining.

The three basic quantities chosen here is an equivalent form to the choice of the exact scaling system. That is why the scaling factor for the material properties here are the same with the corresponding term listed in Table 7.1. It is necessary to point out that the scaling laws in that exact scaling system keeps the energy density conserved between the original system and the scaled system.

This dimensionless analysis was also applied to the simulation of gas-particle flows for dense particle regions by Radl [227]. This work is continued by Nasato [228] who extended the analysis for the Hertz model and a limited analysis in the cohesive contact model.

\subsubsection{Modification of interaction laws}

Some researchers focused on the scaling of model parameters in contact interaction laws to produce scale independent predictions in numerical simulations. Thakur [229] investigated the scaling law for cohesionless and cohesive solids under quasi-static simulation of confined compression and unconfined compression to failure. The work of Yun [230] suggests the scaling rule for static liquid bridge model to reasonably simulate the fluid-like behaviour of particles.

This type of approach is even more a one-sided methodology which cannot take the entire features of the whole system into consideration. Different scaling laws for just one quantity may be obtained by this method. We can find this situation in [229] for cohesive systems, where different equations for inter-particle forces are proposed, leading to a contradiction conclusion that the cohesive force should be scaled linearly, squarely or cubically with the radius of the particle respectively based on different interaction laws.

Consider the adhesive force $f_{0}$ that can be calculated based on three different equations.

1) From the equation,

$$
f_{0}=\frac{A R}{6 s^{2}}
$$

where $A$ is the Hamaker's constant, $s$ is the separation distance between the particles.

The author only realised that the radius of the particle $R$ is a scale related quantity while ignored the scale factor for the distance $s$. It is suggested that the adhesive force should be linearly proportional to the radius of the particle.

Considering the dimension of distance $s$, the force should be scaled squarely proportional to the radius of the particle.

2) From the equation

$$
f_{0}=\frac{4 \pi R^{2}}{\phi k} \sigma_{t}
$$

where $\phi$ is the packing fraction, $k$ is the coordination number and $\sigma_{t}$ is the tensile strength. 
Based on this equation, it is suggested that the force should be scaled with the square of the particle radius, which is correct as all the quantities apart from $R$ in the equation are dimensionless.

3) From the equation

$$
f_{0}=f_{g}=\frac{4}{3} \pi R^{3} \rho g
$$

This suggests that the force should be scaled cubically with the radius of the particle. The dimension of gravity $g$ is neglected because the author regards the radius $R$ as the only factor which has an influence on the cohesive force with upscaling.

The simulation results based on the three methods above show that the quadratic scaling produces very similar behaviour between the original system and the scaled system which demonstrates again that the force should be scaled by a factor $h^{2}$.

\subsection{Concluding remarks}

In this chapter, some existing coarse graining methods have been analysed by the exact scaling law. The basic principle to guarantee the original system equivalent to the scaled system is to make the equation of motion for each system proportional to each other. In the exact scaling system, it can be achieved by simply scaling each quantity involved by a scale factor which can be easily derived according to its dimension. The technique used in the representative model is to keep all the quantities with the same values in both the original system and the scaled system. Then the equation of motion for the scaled system is scaled by a factor proposed artificially based on different assumptions. Unreasonable assumptions easily lead to an inappropriate scale factor. The dimensionless analysis reaches this goal by extending the equation of motion based on the specific interaction laws used in different applications and proposing different dimensionless parameters accordingly. This procedure is complicated sometimes and may not be applied directly to other applications. The method of modifying the interaction law has not considered the scaling problem from this basic principle. The scale factor of the force is proposed only based on the relationship between the force and the particle size of the specific interaction law. 


\section{Chapter 8}

\section{Effective thermal properties of particulate phase change materials}

\subsection{Introduction}

The use of renewable energy, such solar energy has received much attention worldwide with the increasing pressure of energy shortage and environmental pollution in recent years. To eliminate the time discrepancy between the energy production and consumption, it is necessary to improve the thermal energy storage (TES) techniques. Generally, solar heat energy can be stored in different ways including sensible heat, latent heat, or thermochemical reaction heat. In latent heat storage system, the thermal energy is stored when the phase change material (PCM) undergoes a phase change, usually from solid to liquid. With the advantages of high density of energy storage and narrow range of the operational temperature, the latent thermal energy storage is a particularly attractive technique.

The PCM needs to be contained properly for the successful utilization of the latent thermal energy storage system. The most common type of PCM containment is macro-encapsulation in which a significant quantity of PCM is packaged in tubes, pouches, spheres, panels or other receptacles [231]. An undesirable property of PCM is their relatively low thermal conductivity. Therefore, it is needed to increase surface/volume ratio to improve the heat transfer rate. The packed bed TES system is developed by packing a large number of small PCM particles prepared by micro-encapsulation techniques with the heat transfer fluid [232]. This packed bed TES system can be found in a variety of applications, such as heating and cooling systems in buildings [233], solar thermal energy storage [234], solar cooling [235] and compressed air energy storage [236].

Since its wide applications, understanding the nature of the packed bed TES system has long been of a keen research interest. The main research methods in the previous investigations contain experiment $[237,238]$ and numerical calculation. Considering the technological and economical barriers of the experimental works, numerous contributions have been made in the 
field of numerical models to study the performance of this system. These numerical models can be mainly divided into two groups: single-phase model and separate-phase model. In the single-phase model [239], the packed bed is regarded as a quasi-homogeneous medium; and in the separate-phase model [240], the solid and fluid phases are considered separately with two energy conservation equations and coupled by a heat exchange term.

An extensive comprehensive revision of different numerical models of a PCM packed bed system can be found in the review [241]. Fixed grid method [242] and adaptive mesh method [243] are adopted to solve this kind of boundary value problems for partial differential equations, where phase boundary can move with time. The solutions of the differential equations was realized by finite-difference or finite element approximation [244]. It can be realised that the solid material (granular phase changing composites obtained by micro or macro-encapsulating PCMs) of the packed bed TES system is approximated as continuous phase in the previous studies while it is actually the discontinuous particulate system. Therefore, it is necessary to investigate this problem from the discontinuous point of view.

Since it was originated in the 1970s by Cundall and Strack [34], the discrete element method (DEM) has emerged as a reliable and effective numerical technique to model scientific and engineering problems involving particulate systems. Despite its extensive utilizations in the field of mechanical properties of the dynamic particulate system, DEM can also be used to simulate the heat transfer in granular assemblies. Hunt [245] first introduced DEM to solve heat transfer problems of granular material flows and determined the effective thermal conductivity and self-diffusivity. Vargas et al. developed the Thermal Particle Dynamics (TPD) method based on DEM to study the heat conduction in granular materials [246] and stress effects on the conductivity of particulate beds [247]. Chaudhuri et al. [248] simulated heat transfer in granular flow in rotating vessels. Granular flow and heat transport properties are taken simultaneously into account to understand their effect on dryer and calcination performance.

A simple particle-particle heat transfer model is used in the above researches to account for the thermal effects and inevitably suffers from poor accuracy. Feng et al. [249] proposed the discrete thermal element method (DTEM) for accurate and effective modelling of heat conduction in individual particles as well as particulate systems in $2 \mathrm{D}$ cases. This method was extended to a simplified version, termed the pipe-network model, which significantly simplifies the solution procedure of the original DTEM [250]. These studies and others, have demonstrated the capability of the DEM as a powerful tool for investigating heat transfer problems of particulate system [251] and determining the corresponding effective thermal conductivity [252-254]. However, little effort has been made to investigate the particulate system with phase change materials.

The main objective of this paper is therefore to develop an enthalpy based discrete thermal formulation that can take both thermal conductivity and phase change into consideration. The equivalent thermal properties of bulk particle materials with phase change will also be derived based on the combination of a multi-scale modelling scheme and the classic one-phase 


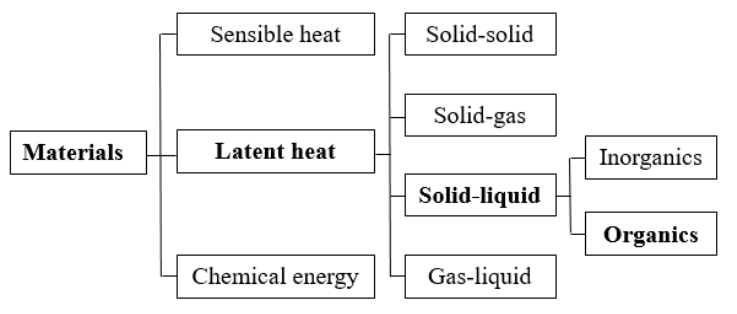

(a) Classification of energy storage materials

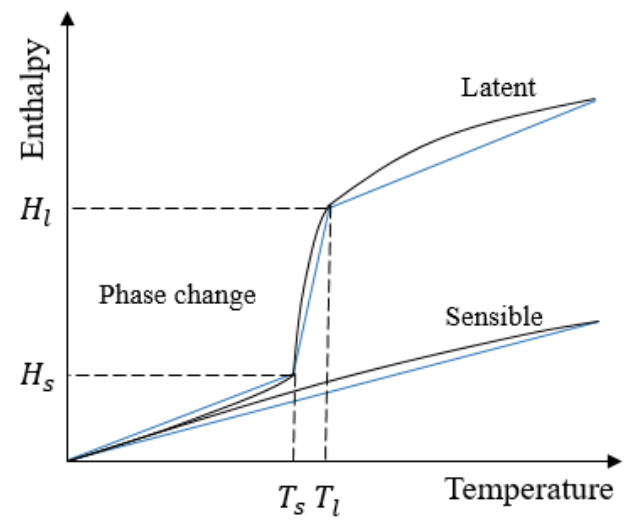

(b) Enthalpy variation with temperature

Figure 8.1: Energy storage materials

Stenfan melting problem.

\subsection{Phase change material}

Thermal energy storage is an important technology to balance the supply and demand of energy. It has been widely used in the applications such as building, concentrated solar plants and thermal management of batteries [255]. The TES systems can be sensible, latent or chemical heat storage as shown in Figure 8.1(a). The sensible heat storage applies a temperature gradient to the material to store or release heat. The disadvantage of the sensible heat storage is the low storage capacity which leads to the huge volume of the systems. The latent heat storage can provide hign storage density with a small temperature difference between storing and releasing heat. The materials used for latent heat storage are called phase change materials and are characterized by storing a large amount of thermal energy while changing from one phase to other (usually solid-liquid states), at a narrow temperature range which has been regarded as a constant temperature in some research, and presenting high heat of phase change (latent heat) [256]. The enthalpy variation with temperature of both sensible and latent materials are shown in Figure 8.1(b). The enthalpy-temperature relation of real material in nature for all the solid, liquid or phase change period is non-linear as shown in the black line while it's often regarded as linear relation (blue line) for convenience in research and engineering application. PCMs can be classified as organic and inorganic of which the organic material paraffin is the most popular commercial PCM due to its long-term stability, suitable phase change temperature and acceptable price.

\subsubsection{Encapsulation of PCM}

The drawbacks of the PCMs include the low thermal conductivity, leakage, subcooling and flammability. Appropriate methods need to be developed to ensure the successful utilisation of PCMs. The containment methods can be classified as the bulk storage in tank heat 
exchangers, the macro or micro encapsulation. In the encapsulation process, the individual particles or droplets of solids or liquid PCMs (the core) are surrounded or coated with a film of polymeric materials (the shell). This method has the advantage of providing a large heat transfer area and reduce the reactivity of the materials with the outside environment. The characteristic diameter of such granular phase change composites range from few centimeters to few millimeters. GPCC materials offer the advantage of maintaining their macroscopic solid form during phase change which are the solid component of the latent heat thermal energy storage system (LHTES) [257].

\subsubsection{Materials characterisation}

The phase change of most PCMs occurs in a temperature range rather than at a constant temperature. From the heat flow results of the differential scanning calorimeter (DSC) measurements, the apparent heat capacity of granular PCMs is acquired as [242]

$$
C_{a p p}(T)=\frac{q(T)}{m_{P C M} \theta}+C_{p}
$$

where $m_{P C M}$ is the mass of PCM used in the DSC, $\theta$ is the heating or cooling rate of the DSC measurement and $C_{p}$ is the specific heat of the solid or liquid PCM.

Packed-bed column experiments during charging and discharging modes have been carried out to analyse the granular phase change material [231].

\subsubsection{Mathematical models}

Besides the experimental works, the complex transient nature of the granular PCM system and the high cost of the set up makes necessary the use of numerical models to study it deeply. These numerical models can be mainly divided into two groups: single phase and two phase models [258].

In the single phase model, the solid phase (PCM spheres) and the liquid phase are considered as a unique phase. Ismail and Stuginsky [259] demonstrate that this model is useful in analyzing fixed beds of both high thermal conductivity and thermal capacity in comparison to the working fluid. In the two phase model, solid and liquid phase are regarded separately. And it can be classified into three categories: the concentric dispersion model [235], the continuous solid phase model [260], and the Schumann's model [240].

All four models assume the solid phase as porous material but not as medium comprised of individual particles. Thus, the accuracy of these models depends on the effective thermal conductivity of the porous material and the total heat transfer coefficient between the fluid and the solid which are usually determined from empirical correlations. 


\subsection{The enthalpy based DTEM framework}

Considering the limitations of the previous continuous model and the discontinuous nature of granular PCMs, it's a potential direction to simulate the particulate system with PCMs from the discontinuous point of view. Therefore, an enthalpy based discrete thermal modelling framework is developed in the current work. It should be noted that only the solid component (PCM spheres) are considered as the main object of this work is to propose a novel method to treat the heat conduction and phase change transition in the particulate system with PCMs rather than model a specific packed-bed column experiment.

\subsubsection{Heat conduction modelling}

Different mechanisms exist in heat transfer process of particulate system, including: thermal conduction through the solid; thermal conduction through the contact area between two particles; thermal conduction to the interstitial fluid; heat transfer by fluid convection; and radiant heat transfer between the surfaces of particles [261]. According to the work of Batchelor and O'Brien [262], when the interstitial medium is stagnant with smaller thermal conductivity compared to that of the particles, thermal conduction through the contact area plays the dominate role in heat transfer process. Thus, the current work of heat transfer in particulate system with phase change material is focused on contact conductance between particles.

\section{Thermal DEM}

To simulate the heat transfer process, temperature is introduced into the DEM as an additional degree of freedom. For the particulate system with a large number of particles, it's extremely time consuming to determine the temperature distribution of each particle. Thus, each particle has only one temperature in DEM simulations. Heat flow occurs via conduction in the active contacts that connect the two particles ( $i$ and $j$ ) whose temperatures are given as $T_{i}$ and $T_{j}$. The Fourier's law of heat conduction denotes the amount of heat transported across their mutual boundary per unit time as

$$
Q_{i j}=K_{i j}\left(T_{j}-T_{i}\right)
$$

where $K_{i j}$, the contact conductance, is the amount of heat transported per unit temperature difference per unit time. The evolution of the temperature of particle $i$ is given as

$$
\frac{d T_{i}}{d t}=\frac{Q_{i}}{C_{i}}
$$

where $Q_{i}$ is the total amount of heat transported to particle $i$ from its neighbor particles calculated from Equation (8.2) as

$$
Q_{i}=\sum_{j=1}^{N} Q_{i j}
$$


in which $N$ is the number of particles contacted with particle $i$; and $C_{i}$ is the total heat capacity of particle $i$ which is defined as

$$
C_{i}=\rho_{i} c_{i} V_{i}
$$

where $\rho_{i}, c_{i}$ and $V_{i}$ are the density, specific capacity and volume of particle $i$. We can discretise Equation (8.3) by expressing the time derivative using forward finite difference to update the particle temperature as

$$
T_{i\langle t+\Delta t\rangle}=T_{i\langle t\rangle}+\frac{Q_{i}}{C_{i}} \Delta t
$$

There are two criteria that need to be satisfied in this thermal DEM method: (1) The temperature of particle $i$ keeps the same value at different contact points with all the neighbor particles which requires that the resistance to heat transfer inside the particle is significantly smaller than the resistance between the particles. This can be achieved because the contact area is often far smaller than the size of particles. (2) The stability criterion of the explicit scheme requires that the temperature of each particle should change slowly enough so thermal disturbances do not propagate further than its immediate neighbors during one time-step. This can be satisfied by choosing a sufficiently small time-step.

Another aspect considered in modelling of thermal conduction is the thermal expansion and contraction of the particle size. Similar to the previous treatment [263-265], the radius expansion/contraction is considered as

$$
r=r_{0}(1+\beta \Delta T)
$$

where $r_{0}$ is the initial radius of particles at the reference temperature $T_{0}, r$ is the current radius, $\Delta T=T-T_{0}$ is the temperature change, and $\beta$ is the thermal expansion coefficient (assuming constant in the current work).

When the thermal expansion of particles is taken into account, granular materials would exhibit the settling behavior under thermal excitations which needs to be simulated by considering kinematic of particles in conjunction with the thermal modelling. This has two consequences. One is the increase of the packing density and the other is the particle rearrangement. The packing density has a major influence on thermal properties of granular phase change materials as can be seen in Section 8.6.2, while the effect of particle rearrangement on thermal properties is less clear for randomly packed particle configurations.

Nevertheless, the effect of particle size change may not be significant in the thermal modelling of phase change materials as the narrow range of the operational temperature during the charging and discharging processes and the randomness of particle arrangement under thermal excitations. Thus most of the thermal simulations conducted in the current work, unless those mentioned specifically, do not consider the size expansion to reduce the computation time because the required time-step in the thermal DEM simulation is often orders of magnitude larger than the corresponding time-step in the mechanical DEM [252]. The thermal induced 
size change effect will be discussed in detail in Subsection 8.6.4.

\section{Thermal contact model}

To accomplish the above thermal DEM simulation, the contact conductance $K_{i j}$ in Equation (8.2) needs to be determined properly. Contact conductance refers to heat transmit ability of two touching materials. Most work related to this topic can be found in area of microelectronics, aircraft industry, nuclear industry and nano-technologies [266]. The thermal contact model in thermal DEM modelling borrows some mature results from the early work of contact conductance [199, 267]. The approximate analytical solution of the contact conductance between two smooth, elastic spheres is adopted to consider heat transfer process in thermal DEM.

$$
K_{i j}=2 k_{i}\left[\frac{3 F_{n} r}{4 E^{*}}\right]^{1 / 3}=2 k_{i} a
$$

where $k_{i}$ is thermal conductivity of the particle, $r$ is the particle radius, $F_{n}$ is the normal force acting between particles, $E^{*}$ is the effective Young modulus for two particles, and $a$ is the contact radius.

Despite the above relation used in most of the thermal DEM researches [246, 247, 252, 268], a thermal pipe contact model is developed in Particle Flow Code [57] in which heat flows between particles through a one-dimensional pipe. The contact conductance is defined as

$$
K_{i j}=\frac{1}{\varepsilon b}
$$

where $\varepsilon$ is the thermal resistance per unit length, and $b$ is the pipe length.

The determination the contact conductance from above models is based more on an ad hoc manner than on a rigorous theoretical foundation. Feng et al. [249, 250] proposed the pipe-network model fo the modelling of heat conduction in particulate system which provided a more rational and accurate model to represent heat conduction.

In the pipe-network model, one particle can be conceptually represented by a simple starshaped pipe network model in which the center is connected to each contact zone as shown in Figure 8.2.

For each individual pipe $j$ of the $i$ th particle, the corresponding thermal resistance $R_{i, j}$ is given by

$$
R_{i, j}=\frac{1}{\pi k_{i}}\left(-\ln \alpha_{i, j}+\frac{3}{2}+\frac{\alpha_{i, j}^{2}}{36}+\frac{\alpha_{i, j}^{4}}{2700}+\frac{\alpha_{i, j}^{6}}{79380}\right)
$$

The thermal contact conductance $K_{i j}$ between the $i$ th and $j$ th particles is formulated as

$$
K_{i j}=\frac{1}{R_{i, j}+R_{j, i}}
$$

A similar approach can also be developed to define the thermal contact conductance for contacting spheres. 


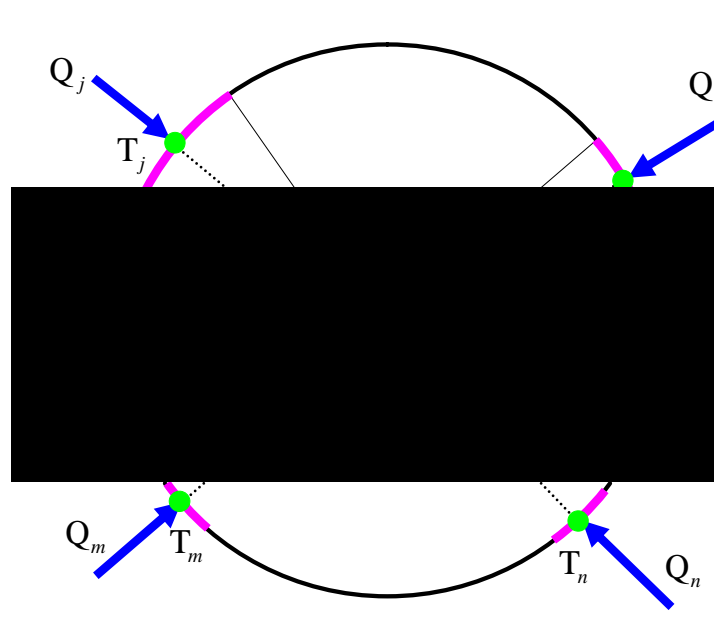

(a) Thermal discrete element

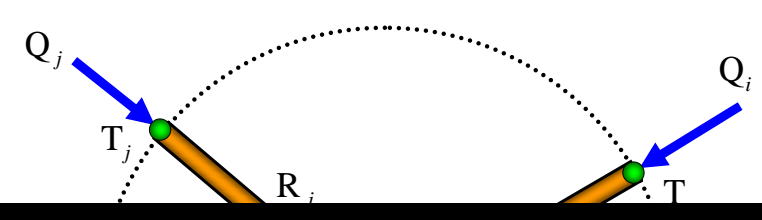

Figure 8.2: Discrete thermal element method [249, 250]

\subsubsection{Phase change modelling}

Phase change materials are viewed as latent heat storage units as a large amount of energy is absorbed or released when materials change from solid to liquid and vice versa. In the DTEM method, the phase change transition is taken into account by using the enthalpy concept. The change in enthalpy equals to the heat absorbed or released for the enclosed system at a constant pressure. The quantity of thermal energy possibly stored depends on the enthalpy variation in the working temperature range.

In the current DTEM framework, a PCM sphere is regarded as a phase change material as shown in Figure 8.1, and the phase change process occurs at the temperature $T_{s}$ and ends at the temperature $T_{l}$ as shown in Figure 8.1(b), and the enthalpy variation is equal to the latent heat $L$. The enthalpy of each sphere is $H_{i}$ and can be obtained by

$$
H_{i\langle t+\Delta t\rangle}=H_{i\langle t\rangle}+Q_{i} \Delta t
$$

For the phase change transition from solid to liquid, the onset enthalpy is $H_{s}=C_{i} T_{s}$ and the end enthalpy is $H_{l}=H_{s}+L$ as shown in Figure 8.1(b).

The specific capacity $c_{i}$ and thermal conductivity $k_{i}$ of each particle need to be modified according to the enthalpy $H_{i}$ of that particle as follows

$$
c_{i}= \begin{cases}C_{s}, & H_{i} \leq H_{s} \\ C_{s}+\frac{H_{i}-H_{s}}{H_{l}-H_{s}}\left(C_{l}-C_{s}\right), & H_{s}<H_{i}<H_{l} \\ C_{l}, & H_{i} \geq H_{l}\end{cases}
$$


and

$$
k_{i}= \begin{cases}k_{s}, & H_{i} \leq H_{s} \\ k_{s}+\frac{H_{i}-H_{s}}{H_{l}-H_{s}}\left(k_{l}-k_{s}\right), & H_{s}<H_{i}<H_{l} \\ k_{l}, & H_{i} \geq H_{l}\end{cases}
$$

To track the phase change evolution of a particulate system, the phase change time $T_{i c}$ of each particle is recorded. A phase transition is assumed to occur at the time when a particle has stored or released a certain amount of the latent heat, or the phase transition latent heat $H_{p t}(\alpha)$ determined by a given parameter $\xi \in[0,1]$ as

$$
H_{p t}(\xi)=(1-\xi) H_{s}+\xi H_{l}
$$

With the phase change time of each particle is known, the position evolution of the phase change front in a particulate system can be obtained in DTEM modelling.

\subsection{Effective thermal properties}

In design of a LHTES, it is necessary to properly determine thermo-physical properties including the effective thermal conductivity and other thermal properties of the system. For the particulate system of phase change material, it is not easy to characterise granular phase change composites due to the nature of the material and the heterogeneity of the sample. Small and non-uniform diameters of spherical capsules also complicate the evolution of latent heat in a heat storage system. The current DTEM method makes it possible to numerically obtain the effective thermal properties of a granular PCM system by applying a homogenisation based technique.

\subsubsection{Effective thermal conductivity}

To predict the effective thermal conductivity (ETC), $\mathbf{K}_{\text {eff }}$, of granular materials is an important subject for many scientific and industrial applications involving particulate systems. Various experimental tests and analytical models have been proposed to measure or analyse the ETC of granular materials [269-271]. Compared to these methods, numerical simulations can be employed to obtain heat transfer of granular materials, which makes the numerical simulation using DTEM a powerful means to predict the ETC.

In theory, the ETC of granular assemblies can be determined by solving the equation of Fourier's law of heat conduction. Therefore, the heat flux $\mathbf{q}^{\prime \prime}$ and temperature gradient $\nabla T$ need to be obtained from DTEM simulations. A simple way is to obtain them from a boundary value problem where the granular sample is considered as a continuum phase. A more accurate method is to obtain the average heat flux $\left\langle\mathbf{q}^{\prime \prime}\right\rangle$ and temperature gradient $\langle\nabla T\rangle$ of a granular assembly [252], which is analogous to compute the average stress and strain of a particulate system by using the multi-scale method [272-274]. In granular materials, the micro scale is 
taken to be a scale where individual particle responses are measurable, while the macro scale is where the whole assembly is considered as a continuum phase. The following description of using the average heat flux and temperature gradient to predict the ETC of a particle assembly is mainly adopted from [252].

\section{Average temperature gradient}

Consider the steady state heat transfer of a granular system, $\Omega$, where the approximate temperature $\tilde{T}^{i}$ of each particle is expressed as

$$
\tilde{T}^{i}=\nabla T \cdot \mathbf{x}^{i}
$$

in which $\mathbf{x}^{i}$ is the relative coordinate of the particle, and $\nabla T$ is the temperature gradient at the macroscale level where the granular assembly is considered as a continuum phase.

Because of the fluctuation of the particle temperature distribution in the particulate system, the real temperature $\hat{T}^{i}$ is not exactly match the approximate temperature $\tilde{T}^{i}$, so

$$
\hat{T}^{i}-\nabla T \cdot \mathbf{x}^{i} \neq 0
$$

To find the specific $\langle\nabla T\rangle$, the square sum of the deviations in Equation (8.17) should be minimised. Thus by employing the least-squares approach, $\langle\nabla T\rangle$ can be obtained as a solution of the following linear equations

$$
\sum_{i \in \Omega} \mathbf{x}^{i}\left(\hat{T}^{i}-\langle\nabla T\rangle \cdot \mathbf{x}^{i}\right)=0
$$

\section{Average heat flux}

In the state of heat equilibrium without heat sources, the temperature of each particle remains constant so the total heat exchange through the boundary of the granular assembly, $\partial \Omega$, is zero:

$$
\int_{\partial \Omega} \mathbf{q}^{\prime \prime} \cdot \mathbf{n} d A=\sum_{i \in \partial \Omega} q^{i b}=0
$$

where $\mathbf{q}^{\prime \prime}$ is the heat flux flowing through the boundaries, $\mathbf{n}$ is the outward oriented unit boundary normal and $\int_{\partial \Omega}$ is the integration on the boundary of the particulate system, $q^{i b}$ is the total heat flowing through the boundary contacting with particle $i$.

The average heat flux $\left\langle\mathbf{q}^{\prime \prime}\right\rangle$ of the particulate system is given by

$$
\left\langle\mathbf{q}^{\prime \prime}\right\rangle=\frac{1}{V} \int_{\Omega} \mathbf{q}^{\prime \prime} d V=\frac{1}{V} \sum_{i \in \partial \Omega} q^{i b} \mathbf{x}^{i b}
$$

where $V=|\Omega|$ is the volume of the particulate system and $\mathbf{x}^{i b}$ denotes the coordinates of the contact point between particle $i$ and the boundary. Hence, the average heat flux of the 
particulate system can be computed from the simulation.

\section{Effective thermal conductivity}

By applying Fourier's law of heat conduction, the effective thermal conductivity $\mathbf{K}_{\text {eff }}$ should satisfy

$$
\mathbf{K}_{\text {eff }}\langle\nabla T\rangle=-\left\langle\mathbf{q}^{\prime \prime}\right\rangle
$$

$\mathbf{K}_{\text {eff }}$, as a tensor, is required to be symmetric and positive definite which are automatically satisfied by enforcing the symmetry of $\mathbf{K}_{\text {eff }}$ [275]. Then $\mathbf{K}_{\text {eff }}$ is obtained from solving the following overdetermined linear system of equations

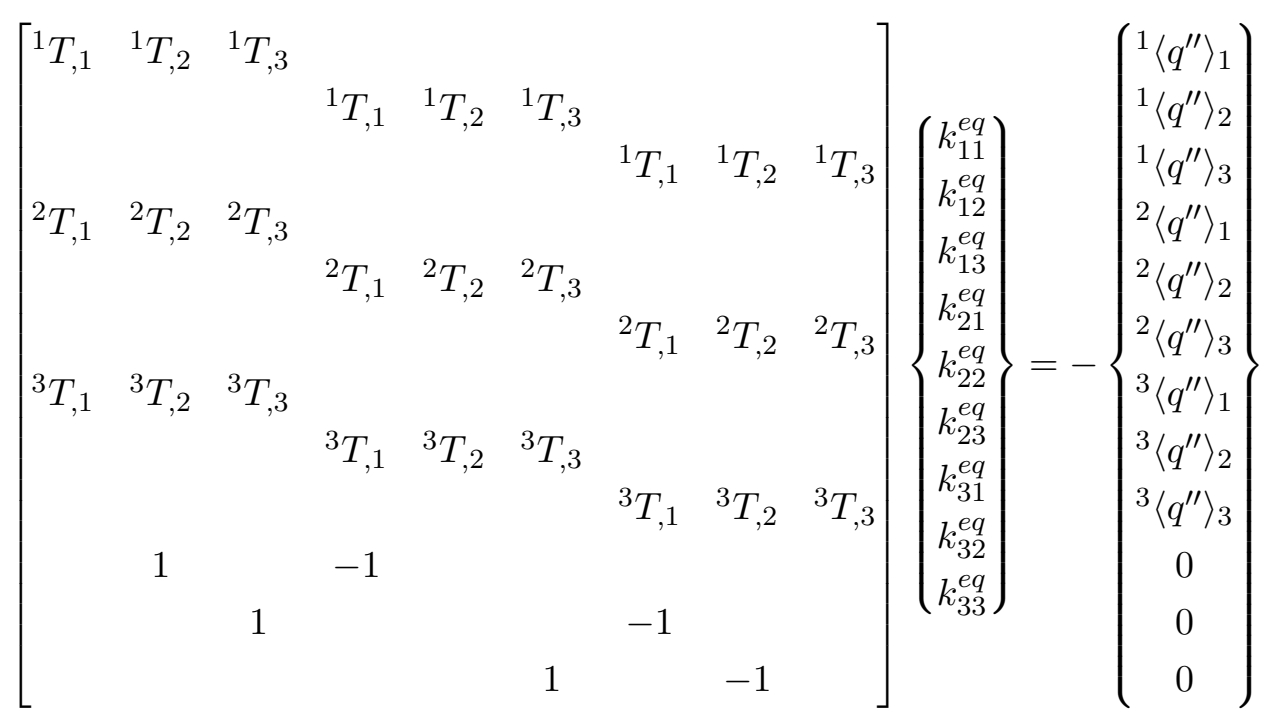

where all blank coefficients of the matrix are 0 and the superscripts 1, 2 and 3 represent the boundary problems in the three directions of the Cartesian coordinate system. This equation is solved as a linear least-squares problem.

\subsubsection{Effective latent heat}

The effective latent heat $L_{\text {eff }}$ of the particulate system of PCMs is an important property which indicates its heat storage capacity. For a granular system, the effective latent heat $L_{\text {eff }}$ can be simply obtained as

$$
L_{\mathrm{eff}}=L \nu_{s}
$$

where $L$ is the latent heat of the granular particles, and $\nu_{s}$ is the solid volume fraction, or packing density, of the particulate system. 


\subsection{Validation}

As our current work is to treat granular PCMs from the discontinuous point of view, its validity will be demonstrated by applying it to solve a discrete/particle version of the classical phase change problem - the Stefan problem, where a particulate system with PCMs will be treated as a one-dimension continuous medium. The interface evolution of the particulate system should be analogous to its continuum counterpart. For the continuum phase, the phase transition is described as a boundary value problem of partial differential equations (PDE), where the phase boundary moves with time. In the following subsections, the moving boundary problem of continuum-the Stefan problem is introduced first. The latent heat is an important parameter which affects the movement of the phase boundary (solid-liquid interface) of the system. The validity of the DTEM method will be demonstrated by comparing the actual effective latent heat $L_{\text {eff }}$ of the particulate system with the fitted one $L_{\text {eff-fit }}$ derived from the numerical results.

\subsubsection{The Stefan problem}

This classical moving boundary problem dates back to the study of the melting of glaciers by the Slovenian physicist Jozef Stefan in 1889 [276]. It aims to describe the temperature distribution in a homogeneous medium undergoing a phase change. The physical constraints of this problem are the conservation of energy and the local velocity of the interface depends on the heat flux discontinuity at the interface. From a mathematical point of view, the phases are regions in which the solutions of the underlying PDE are continuous. The moving boundary are infinitesimally thin and the PDE are not valid at phase change interfaces. Therefore, an additional Stefan Condition is needed to obtain closure. The analytical solutions for this problem is available for one-dimensional cases of an infinite region with simple initial and boundary conditions.

\section{Formulations}

For a semi-infinite region, the initial temperature is $T_{0}$. At time $t>0$, the temperature of the boundary at $x=0$ is suddenly kept at $T_{w}$. The governing equations for the temperature distribution $T(x, t)$ in the region are formulated as follows

$$
\begin{gathered}
\left\{\begin{array}{lr}
C_{l} \frac{\partial T}{\partial t}=\frac{\partial}{\partial x}\left[k_{l} \frac{\partial T}{\partial x}\right] & 0<x<s(t), t>0 \\
T_{l}(0, t)=T_{w} & t>0
\end{array}\right. \\
\left\{\begin{array}{lr}
C_{s} \frac{\partial T}{\partial t}=\frac{\partial}{\partial x}\left[k_{s} \frac{\partial T}{\partial x}\right] & s(t)<x<+\infty, t>0 \\
T_{s}(+\infty, t)=T_{0} & t>0
\end{array}\right.
\end{gathered}
$$


subject to the initial condition

$$
T(x, 0)=T_{0}
$$

where $s(t)$ is the interface position, and the subscripts $s$ and $l$ indicate the solid and liquid phases.

The moving rate of the interfaces is controlled by the latent heat lost or absorbed at the boundary. The following equation, known as the Stefan Condition, describes this process.

$$
k_{l} \frac{\partial T_{l}}{\partial x}-k_{s} \frac{\partial T_{s}}{\partial x}=-\lambda \frac{d s}{d t} \quad \text { at } \quad x=s(t)
$$

where $\lambda=\rho L$ is the heat of phase change per unit volume (note that $L$ is the latent heat coefficient) and $d s / d t$ is the velocity of this interface.

Moreover, the temperature verifies:

$$
T_{l}=T_{s}=T_{m} \quad \text { at } \quad x=s(t)
$$

where $T_{m}$ is the melting temperature.

This problem can be formulated in non-dimensional variables for a finite sheet $0 \leq x \leq l$ ( $l$ is a standard length) by assuming constant thermal values and using a simple scaling [277]. The non-dimensional form makes it convenient to obtain the analytical solution and save computational costs of the numerical solutions.

\section{Analytical solution}

The exact solution of the one-dimensional Stefan problem, available in the literature [278], can be derived by using the similarity variable to transform the governing equation from partial derivatives to an ordinary differential equation. The solution of the temperature distribution is

$$
T(x, t)=T_{0}-\frac{T_{0}}{\operatorname{erf}(\lambda)} \operatorname{erf}\left(\frac{x}{2 \sqrt{t}}\right)
$$

where $\lambda$ is the freezing constant given by the root of the following transcendental equation

$$
\lambda e^{\lambda^{2}} \operatorname{erf}(\lambda)=S t e / \sqrt{\pi}
$$

in which $\operatorname{erf}$ is the error function and Ste is the Stefan number defined as

$$
\text { Ste }=\frac{C \Delta T}{L}
$$

where $C$ is the specific heat of solid phase in the freezing process, but is the specific heat of the liquid phase in the melting process; $\Delta T$ is the temperature difference between the two phases. The moving front position is expressed as

$$
s(t)=2 \lambda \sqrt{\nu t}
$$


where $\nu=k_{s} / C_{s} \rho$ is the heat diffusivity.

\subsubsection{Effective latent heat of a particle system based on DTEM}

As mentioned in Section 8.3.2, the phase change time of each particle can be recorded during the simulation. The time history of the phase change times and positions of all the particles in the system will depict how the melting interface of the system evolves with time. If the particulate system is regarded as an equivalent continuum, it should have a similar relation as described by Equation (8.32). The phase change time - position points of the particles can be curve fitted by this relation, and then the resulting freezing constant $\lambda$ can be used to derive the effective latent heat based on both Equation (8.30) and Equation (8.31). Thus, the fitted effective latent heat $L_{\text {eff-fit }}$ is derived based on the simulation results as

$$
L_{\text {eff-fit }}=\frac{C \Delta T}{\lambda e^{\lambda^{2}} \operatorname{erf}(\lambda) \sqrt{\pi}}
$$

If the proposed enthalpy based DTEM method for simulations of problems with PCMs is valid, the $L_{\text {eff-fit }}$ obtained by Equation (8.33) should be close to $L_{\text {eff }}$ calculated by Equation (8.23). Numerical simulations using the enthalpy based DTEM method are conducted to derive the fitted effective latent heat $L_{\text {eff-fit }}$ of a particulate system with granular PCMs. Three cuboid shaped boxes with $1 \mathrm{~m}$ in both length and width and $5 \mathrm{~m}$ in height filled with PCM capsules have been randomly generated with some initial contacts between neighbouring particles. These samples have different packing densities $\nu_{s}=0.6,0.65$ and 0.7 respectively. The diameters of the capsules have a uniform distribution between $0.05 \mathrm{~m}$ to $0.06 \mathrm{~m}$.

The top and bottom of each box have a constant temperature of $100^{\circ} \mathrm{C}$ and $60^{\circ} \mathrm{C}$ respectively. Heat is conducted between the top and bottom of the box with the contacting particles in the sample. The side surfaces of the boxes are adiabatic or insulated. The initial temperature of all the PCM capsules is $60^{\circ} \mathrm{C}$. It is assumed that the particles are ideal phase change materials and the melting process starts at a temperature point $T_{m}=60^{\circ} \mathrm{C}$ rather than a temperature range. The phase transition latent heat $H_{p t}$ is set by choosing the parameter $\xi=0.5$. A particle is assumed melted when the enthalpy of the particle $H \geq H_{p t}$. The thermal properties of the PCM capsules are set as $C_{s}=4 \mathrm{~W} \cdot \mathrm{m}^{-1} \cdot \mathrm{K}^{-1}, C_{l}=8 \mathrm{~W} \cdot \mathrm{m}^{-1} \cdot \mathrm{K}^{-1}$, and $L=10 \mathrm{~kJ} / \mathrm{kg}$. The thermal simulation is performed until a steady-state heat transfer is achieved for the particulate system. The particle system is then treated as a one-dimensional continuous medium along the vertical (z) direction. The corresponding equivalent thermal properties of the system are obtained.

Figure 8.3 shows the temperature distribution of Sample $1\left(\nu_{s}=0.6\right)$. During the initial heating period, the capsules near the top are heated up while the remaining part keep the initial temperature. As time elapses, the temperature rises gradually in the system.

The phase change times and positions of all the particles in Sample 1 are depicted in Figure 8.4, and their relation is curve fitted using Equation (8.32), from which the freezing constant $\lambda$ is 


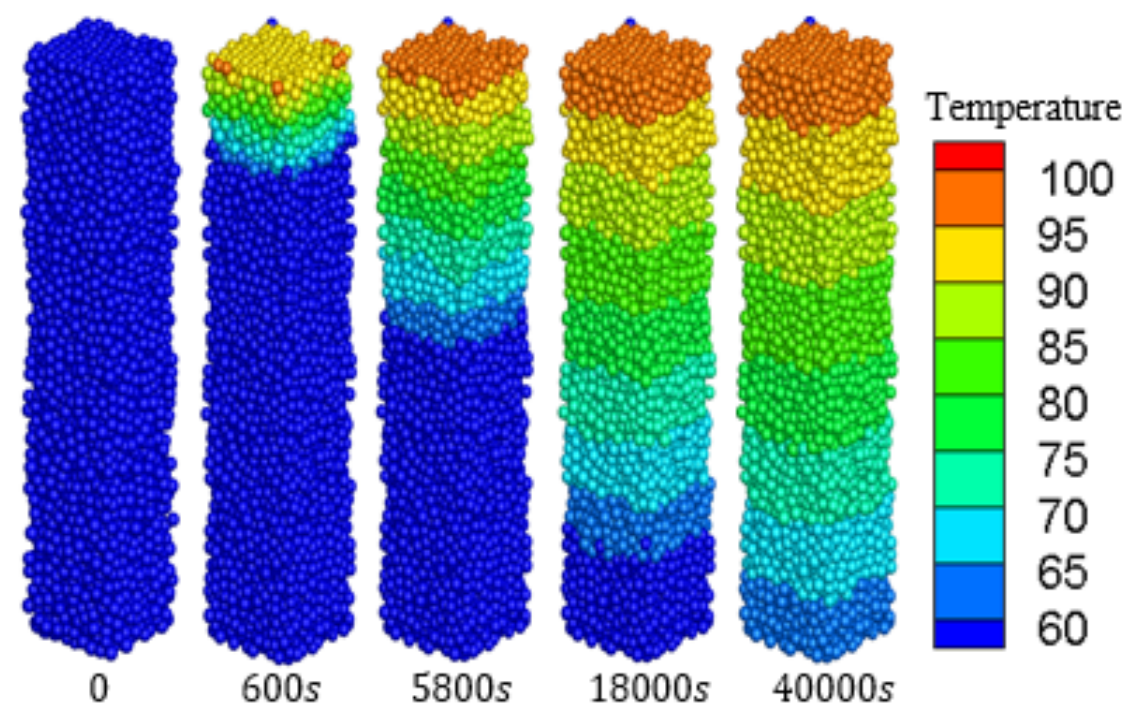

Figure 8.3: Temperature distributions and time evolutions of the particulate system with PCM capsules

obtained as 0.0812. Then, by applying Equations (8.23) and (8.33), the effective latent heat $L_{\text {eff }}$ and the fitted one $L_{\text {eff-fit }}$ can be computed. Both values of $L_{\text {eff }}$ and $L_{\text {eff-fit }}$ for all the three samples are listed in Table 8.1. It can be seen that the fitted values of the effective latent heat $L_{\text {eff }}$ match with the actual values of the effective latent heat very well, thus demonstrating the validity of the DTEM method.

\subsection{Further Illustrations}

Further simulations are performed to show the effectiveness of the DTEM method in modeling heat transfer in particulate systems involving phase change materials.

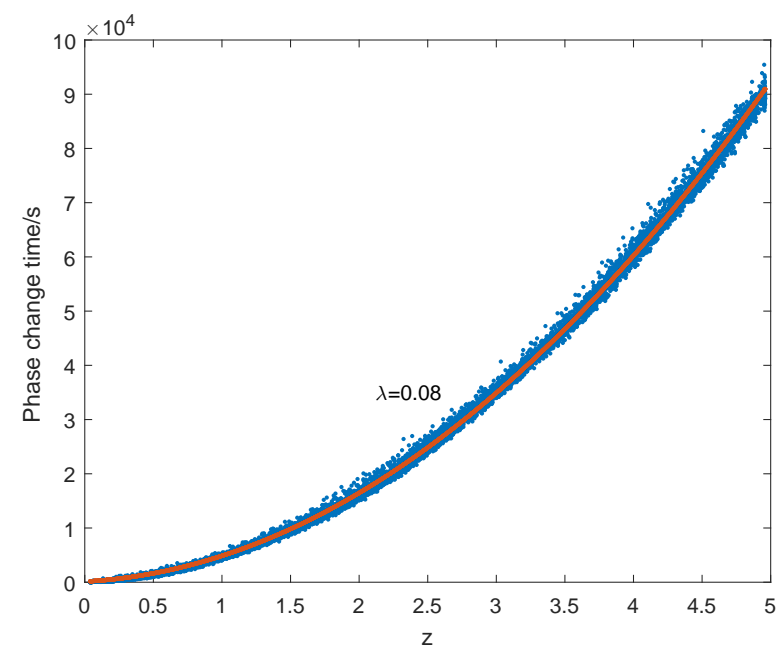

Figure 8.4: Determination of the effective latent heat (Sample 1) 
Table 8.1: Effective latent heat of the particulate system

\begin{tabular}{c|c|c|c}
\hline Sample & 1 & 2 & 3 \\
\hline Packing Density $\nu_{s}$ & 0.6 & 0.65 & 0.7 \\
Freezing Parameter $\lambda$ & 0.0812 & 0.0789 & 0.0761 \\
$L_{\text {eff }}(\mathrm{kJ} / \mathrm{Kg})$ - Eq.(8.23) & 6.0 & 6.5 & 7.0 \\
$L_{\text {eff-fit }}(\mathrm{kJ} / \mathrm{Kg})$ - Eq.(8.33) & 6.027 & 6.404 & 6.880 \\
\hline
\end{tabular}

\subsubsection{Temperature evolution}

Another thermal simulation is conducted using Sample 1 where the granular capsules are considered as sensible material by setting the latent heat $L$ to be 0 . Figure 8.5(a) shows the temperature profile of Sample 1 at different heights for various time instances. A similar profile for the phase change material used in the previous simulations is also displayed in Figure 8.5(b) for comparison. The top $(\mathrm{z}=0 \mathrm{~m})$ and bottom $(\mathrm{z}=5 \mathrm{~m})$ boundaries of Sample 1 have a constant temperature of $100^{\circ} \mathrm{C}$ and $60^{\circ} \mathrm{C}$ respectively. The initial temperature of all the capsules in the sample is $60^{\circ} \mathrm{C}$. The heat flux flows from the top to the bottom through the granular capsules. The temperature of the capsules increases during the heat-absorbing process. Heat conduction between two capsules occurs because of their temperature difference.

The phase change material has the ability to absorb large amount of heat without changing its temperature. Thus it takes longer for heat to transfer from the top to the bottom of the phase change materials in the sample. Fig. 8.5 (a) shows that at the time 600s, the heat transfer process is almost completed in the whole sample for the sensible materials; while Fig. 8.5(b) indicates that only just over $1 / 10$ of the sample o phase change materials is affected by the heat transfer at the same time. Also, the sample with sensible material reaches the steady-state temperature distribution in about $800 \mathrm{~s}$, while for the system with phase change materials, due to the notable heat storage capacity, the same process takes more than $18000 \mathrm{~s}$, which is more than 20 times that of the sensible one.

This obvious significance can also be observed from the temperature evolution of the particles at different heights as shown in Figure 8.6. Capsules at five different heights are selected and their temperatures are depicted against time. For the capsules of sensible material, the temperature increases linearly and reaches to the final temperature very quickly. For the capsules of phase change material, it shows a non-linear relation between temperature and time. The PCM capsules maintain in the melting temperature $60^{\circ}$ for a long time because of the high capacity of storing the latent heat.

\subsubsection{Effect of capsule size and density}

Additional two samples with PCM capsules with different radius ranges but a constant packing density $\nu_{s}=0.6$ are generated. The resulting three samples are termed as small $(0.04-0.05 \mathrm{~m})$, middle $(0.05-0.06 \mathrm{~m})$ and large $(0.06-0.07 \mathrm{~m})$. Figure $8.7(\mathrm{a})$ show the total melt fraction of the 


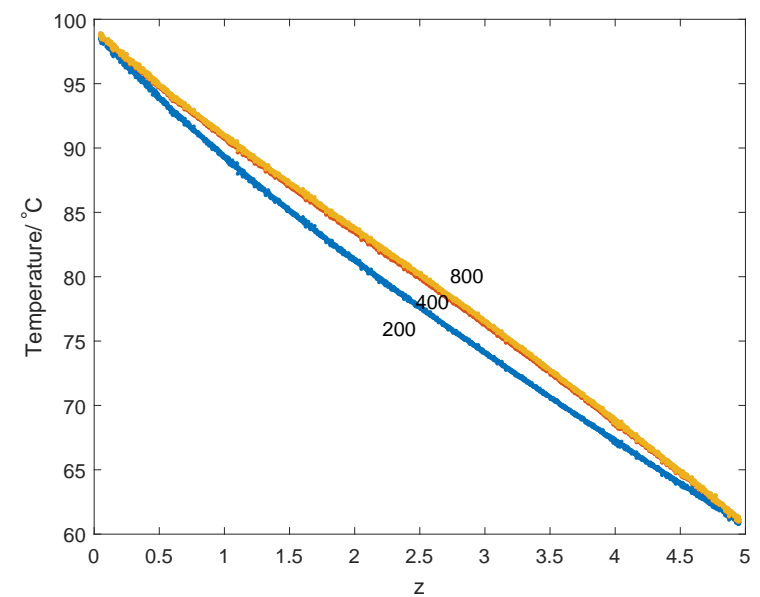

(a) Sensible material

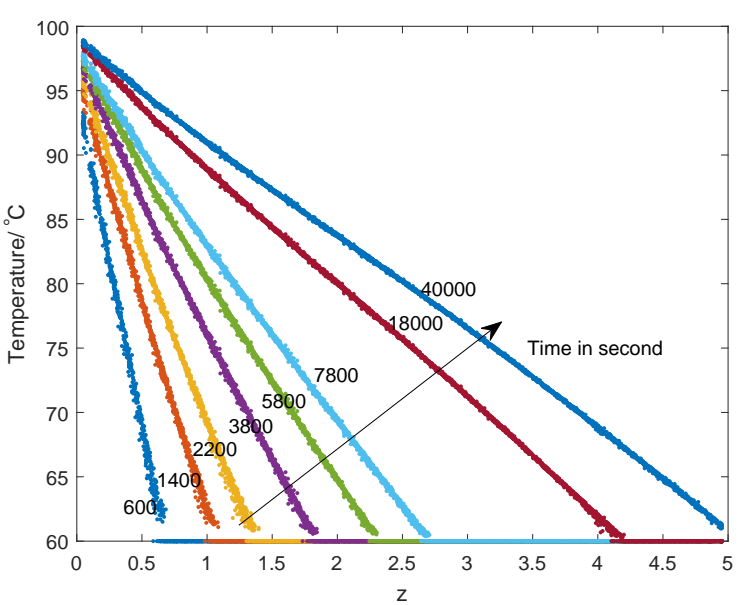

(b) Phase change material

Figure 8.5: Temperature profiles and evolutions of the particulate system with two different materials at different time instances

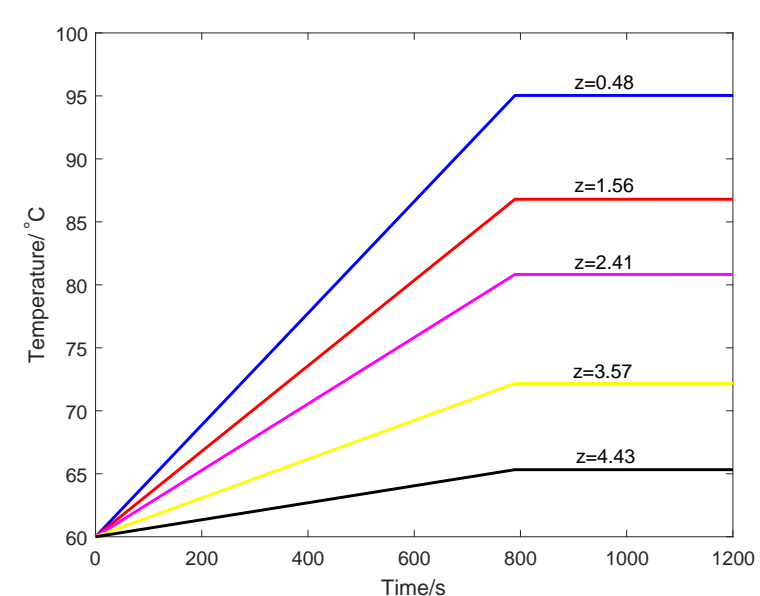

(a) Sensible material

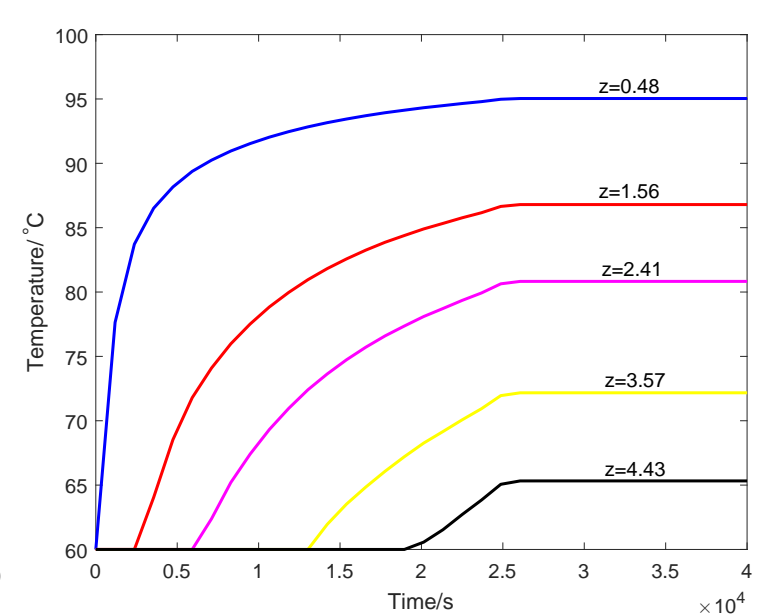

(b) Phase change material

Figure 8.6: Temperature evolutions of the particulate system with two different materials at different heights

particulate system with different capsule sizes. It can be seen that the samples with smaller capsules taking less time for charging which indicates the same phenomenon as pointed in the previous research [279], but the effect is weak here. This difference is caused by the dissimilarity of the packing configuration of the samples with different particle sizes. The detailed explanation can be found in the work of [220, 221]. Figure 8.7(b) shows the evolutions of the total melt fraction of the three samples with different packing densities. The difference between these cases is obvious. The sample with the largest packing density attains the complete melting position quickest. The time needed for the loosest sample to reach the final state is more than three times than that of the densest sample. This is because that a denser sample has a larger effective thermal conductivity which can be seen in Table 8.2 below. 


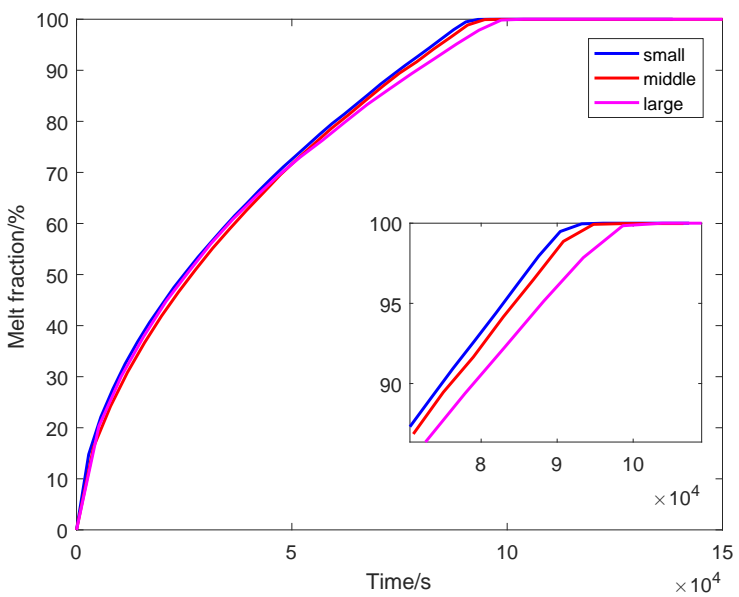

(a) Different particle sizes

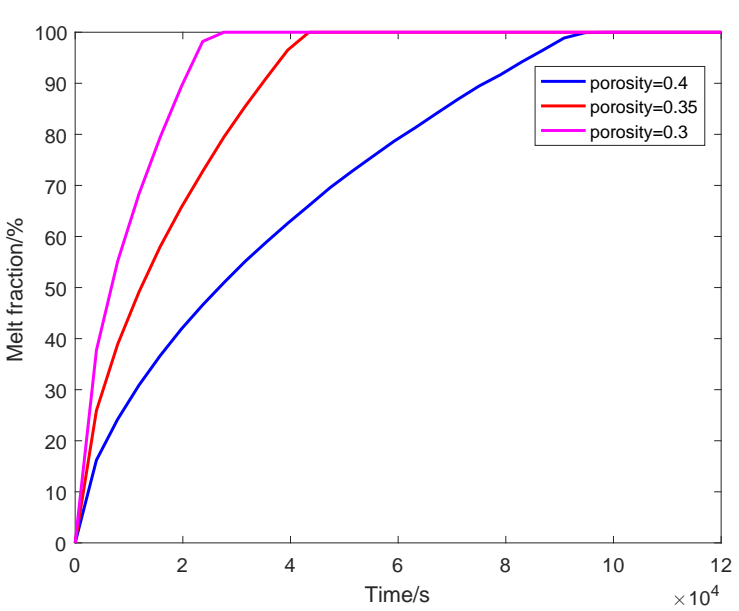

(b) Different packing densities

Figure 8.7: Melt fraction evolutions of the particulate system with different particle sizes and packing densities

\subsubsection{Determination of effective thermal conductivity}

As described in Section 8.4, the effective thermal properties can be calculated from the numerical results. Simulations are conducted on five different samples with different particle size distributions or packing densities as shown in Table 8.2. In the current simulations, the temperatures are specified at the top and bottom boundaries of the samples, and all the other boundaries are isolated. So only the thermal conductivity along the $\mathrm{z}$ direction is determined.

Take Sample 1 as an example. The heat flux at the state of heat equilibrium is obtained when the heat flux at the top and bottom of the sample are equal. Thus, the average heat flux $\left\langle q^{\prime \prime}\right\rangle=374.44 \mathrm{Wm}^{-2}$ is shown in Figure $8.8(\mathrm{~b})$. The average temperature gradient is determined by fitting the temperature distribution using a linear relation which leads to $\langle\nabla T\rangle=6.92 \mathrm{~K} / \mathrm{m}$ as shown in Figure 8.8(a). Then, by using Equation (8.21), the effective thermal conductivity is computed as shown in Table 8.2. The effective thermal conductivity of the other samples are calculated by the same method and listed in Table 8.2. It can be observed that the effective thermal conductivity is affected significantly by the packing density of a sample while the particle size distribution has a minor effect on the conductivity.

Table 8.2: Effective thermal conductivity of the particulate system

\begin{tabular}{c|c|c|c|c|c}
\hline Sample & 1 & 2 & 3 & 4 & 5 \\
\hline Packing density $\nu_{s}$ & 0.6 & 0.65 & 0.7 & 0.6 & 0.6 \\
Radius $r / \mathrm{m}$ & $0.05-0.06$ & $0.05-0.06$ & $0.05-0.06$ & $0.04-0.05$ & $0.06-0.07$ \\
\hline$K_{\text {eff }}(\mathrm{W} / \mathrm{mK})$ & 54.08 & 137.95 & 236.66 & 55.85 & 48.61 \\
\hline
\end{tabular}




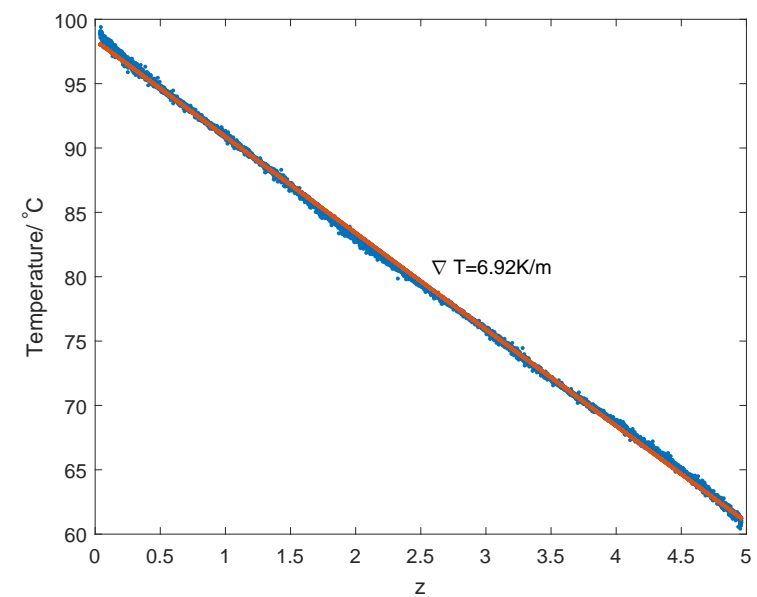

(a) Final particle temperature distribution and fitted linear temperature distribution with computed gradient

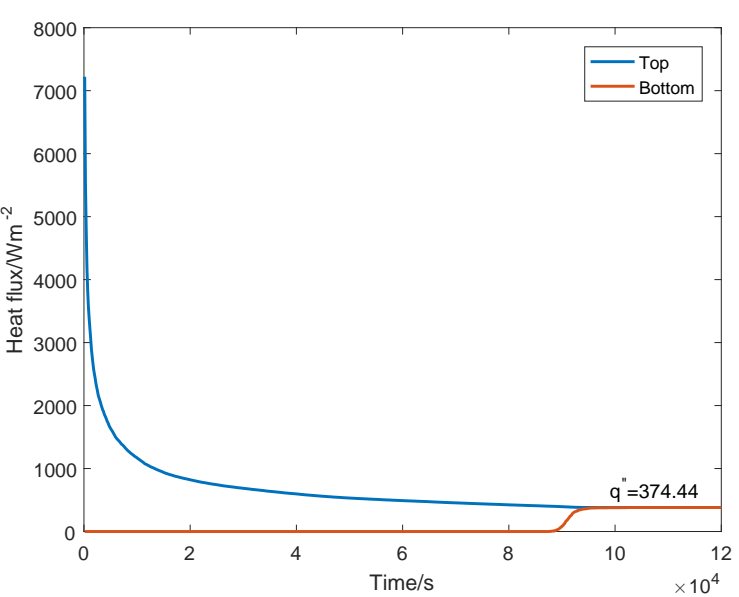

(b) Time histories of heat flux at the boundaries

Figure 8.8: Steady-state particle temperature distribution (a) and heat flux evolutions at the boundaries (b) for Sample 1

\subsubsection{Effects of thermal induced particle size and packing configuration changes}

The size change of particles due to thermal expansion will cause the increase of packing density and the particle rearrangement of the granular system. The consequence of particle rearrangement induced by the thermal excitation may be similar to that caused by difference packing configurations.

Three particle packings are generated using the same parameters as in Sample 2. The only difference between these packings is that they are generated using different random seeds. The same thermal simulations are conducted on these three packings with or without considering the thermal induced size expansion. The particle size expansion is considered following Equation (8.7). The thermal expansion coefficient is set to be $\beta=10^{-4} \mathrm{~K}^{-1}$ based on [280]. The curve fitted finial steady-state temperature distributions and phase change times of these six cases are shown in Figure 8.9(a) and (b) respectively.

Comparing the phase change times of the same packing with or without size change in Figure 8.9(b), it can be seen that the heat transfer is slightly quicker when the size expansion is taken into account in the simulation, as expected. However, the difference is not significant and comparable with the difference caused by different random packing configurations with the same packing density. Therefore, to reduce the computation time and improve the simulation efficiency, it is a reasonable choice to conduct the thermal modelling without considering the size expansion of particles. 


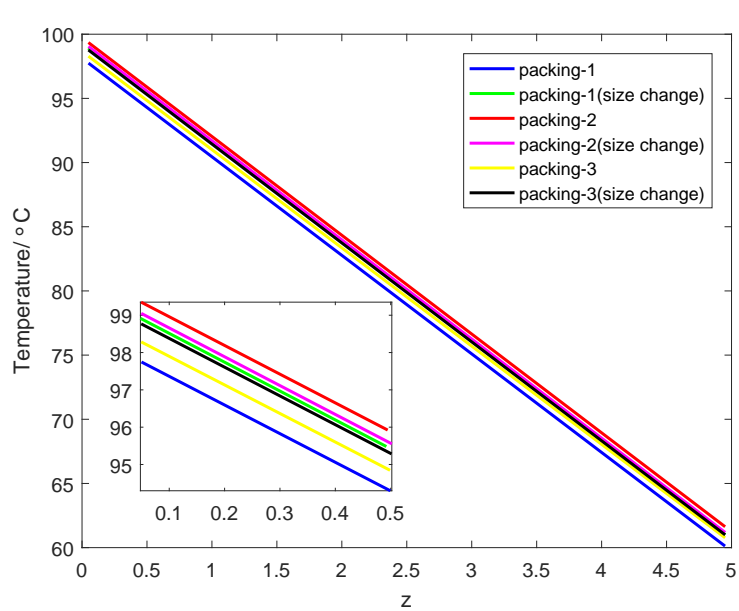

(a) Final temperature distribution

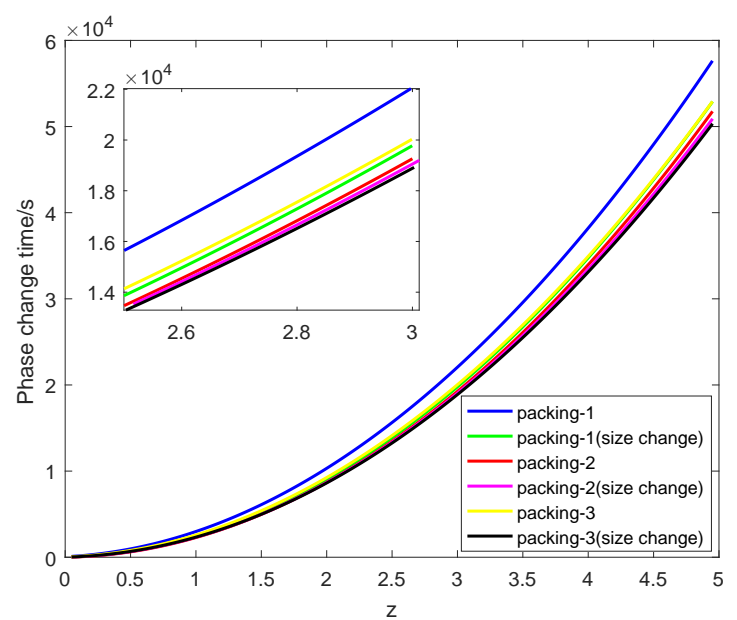

(b) Phase change time

Figure 8.9: Final temperature distribution (a) and phase change time (b) for different packings with or without size change effect

\subsection{Concluding remarks}

This chapter has developed an enthalpy based discrete thermal modelling framework for particulate systems with phase change material which can consider both the heat conduction process and the phase change transition. The proposed algorithm is simple and effective. In addition, the equivalent thermal properties of bulk particle materials with phase change have also been derived based on a simple multi-scale modelling scheme. The proposed methodology is assessed by solving a particle version of the classic one-phase Stefan melting problem. Additional numerical simulations have also been conducted to illustrate the effectiveness of this effective modelling framework.

The effect of capsule size and packing density can be evaluated directly in the current DTEM method. A particle system of phase change materials with smaller capsules or larger packing densities takes lesser time for charging or discharging. The effective thermal conductivity is affected significantly by the packing density while the particle size distribution has a minor effect. The effect of thermal induced particle size change is not predominant when the temperature involved is low and within a narrow range.

It is noted, however, that the current method can only consider the solid component of the heat storage system without considering heat transfer through fluid phases or by convection. In addition, PCM capsules are treated as spheres with a homogeneous property, thus a possible detailed structure of real PCM capsules is not the focus of the current work. 


\section{Part V}

\section{Conclusion}




\section{Chapter 9}

\section{Conclusion}

\subsection{Major contributions}

This thesis is devoted to improve the discrete element method from three different levels considering its current inadequacies in simulating the behaviour of granular materials. Overall, a novel normal contact model has been developed by introducing the statistical GW model which can consider the stochastic surface roughness; a new packing characterisation method has proposed based on the principal component analysis with which the configurations of particle assemblies can be evaluated and compared quantitatively; some existing coarse graining methods have been critically analysed by the exact scaling law; an enthalpy based discrete thermal modelling framework for particulate systems with phase change material has been established and effective thermal properties of particulate phase change materials are derived by the homogenisation method.

The main contributions made in individual chapters/parts are summarised below:

The main content of this thesis is from Chapter 3 and includes three parts. Chapters 3 and 4 are about the development of contact models for rough particles. The theory background and the algorithm development are achieved in Chapter 3 with the following contributions:

1. Developed a normal interaction law based on the GW model.

2. Presented two non-dimensional forms of the model that have a substantial impact on the computational efficiency.

3. Proposed a Newton-Raphson iterative procedure to obtain the contact force with surface roughness parameters.

4. Conducted a simple extension of the classic GW model and deduced a theoretical inconsistency at the limit condition when the surface is smooth.

Several extended GW models have been proposed in Chapter 4, including 
1. Presented a DEM incorporated elastic GW (E-GW) model to treat the contact problem with positive overlap in DEM simulations.

2. Established a normal interaction law by a two-step curve-fitting procedure.

3. Conducted one and three dimension compression tests to investigate the effect of surface roughness on the mechanical behaviour of a particle system.

4. Proposed the elasto-plastic GW (EP-GW) model to allow plastic deformation at the contact asperities

5. Developed a preliminary tangential contact model and a thermal conduct model under the assumptions of surface roughness described in the GW model.

A novel packing characterising method is proposed in Chapter 5 and Chapter 6 which is the second main work of this thesis which contains the following contributions:

1. Established a framework of converting the packing configuration information into data needed in principal component analysis in $2 \mathrm{D}$ and $3 \mathrm{D}$ cases.

2. Analysed some special packings which exploits the additional properties of the principal variances and modes.

3. Conducted comprehensive investigations for several sets of purposefully generated packings to understand relationships of their principal variances with packing features.

4. Revealed the differences between two packings by principal variances (PV) and dissimilarity coefficient (DC).

5. Investigated the effects on a packing caused by configuration randomness, packing density, particle size distribution, uniformity and isotropy.

The third part of thesis, including Chapter 7 and Chapter 8, is devoted to treat multi-scale problems involved in discrete element modelling. The main contributions of this part are as follows.

1. Analysed some existing coarse graining methods by the exact scaling laws.

2. Developed an enthalpy based discrete thermal modelling framework for particulate systems with phase change material.

3. Assessed the proposed methodology by solving a particle version of the classic one-phase Stefan melting problem.

4. Derived the effective thermal properties of phase change materials based on a simple multiscale modelling scheme refer to the analogous homogenisation method proposed in the combined continuous-discontinuous problem. 


\section{$9.2 \quad$ Future work}

The research work presented in this thesis has improved the discrete element method from different perspectives, but some work is still preliminary. Further studies that may be beneficial are suggested as follows.

1. Improved tangential and rolling resistance contact models for rough particles

The extended contact models proposed in the current work mainly focus on the contact force in the normal direction which is due to the assumption in the classic GW model of which the random variance is the height of the asperities in the normal direction. The attempt to extend the model to the tangential direction is preliminary and limited. Further work needs to be done to develop a more complete and reliable tangential contact model as well a rolling resistance contact model which can take the asperities randomness into account. A key point for this issue is to introduce more random factors other than the surface roughness.

2. The advanced stochastic DEM method

Randomness is an unavoidable phenomenon occurring in nature especially for granular materials. Only the randomness of surface roughness is considered in the current work which is far from sufficient. It is worthy to consider other randomness factors and introduce them properly into the DEM method based stochastic or probabilistic mechanics. Such a stochastic DEM will facilitate to have a better understanding stochastic properties of granular materials which may play a fundamental role on the performance of particulate systems.

3. The extended PCA method for packings with particles of variable shapes

The PCA based characterisation method in this thesis is developed for packings with the simplest and most common type of particles - disk/sphere. With the advancement of the DEM method, more complicated particle shapes have been applied thus this evaluating method should also been extended to consider the situation accompanying with it. A general PCA based characterisation method can be established to analyse the particle packings with different particle shapes.

4. The physical basis of the PCA method

The proposed PCA method is developed to evaluate the packings more from the mathematic point of view rather than the physical basis. At the current stage, there still lacks a clear understanding of the relationship between the principal variances and the specific physical properties of a packing. The ultimate purpose of the research is to improve the understanding of some unique features of granular materials, such as dilatancy, liquefaction, phase transformation, critical state, shear band, jamming process and so on. Therefore further work is worth being pursued to understand the physical basis of the PCA method for which the sufficient analysis of experiment or simulation results of real granular materials is essential. 
5. The efficient and accurate DEM method for large scale problems

At the current stage, an efficient and comprehensive DEM method for large scale problems in industry with billions of particles is still lacked. In addition to using high performance computing and/or GPU platforms, tt is of great importance to continuously improve the DEM method by making progress in theories relating the macro- and micro-scales and developing more robust and efficient computer codes.

6. The improvement of the DTEM method for particulate phase change materials

The current DTEM method for particulate phase change materials can only consider the solid component of the heat storage system without considering the heat transfer through fluid or by convection. In addition, PCM capsules are treated simply as spheres with a homogeneous property regardless of the possible detailed structure of real PCM capsules. These inadequacies need to be improved in the next stage. 


\section{References}

[1] HJ Herrmann and Stefan Luding. Modeling granular media on the computer. Continuum Mechanics and Thermodynamics, 10(4):189-231, 1998.

[2] Lawrence E Malvern. Introduction to the Mechanics of a Continuous Medium. Number Monograph. 1969.

[3] Y Kishino. Disc model analysis of granular media. Studies in Applied Mechanics, 20:143-152, 1988.

[4] Charles Augustin de Coulomb. Essai sur une application des règles de maximis 8 minimis à quelques problèmes de statique, relatifs à l'architecture. 1776.

[5] Otto Mohr. Welche umstände bedingen die elastizitätsgrenze und den bruch eines materials. Zeitschrift des Vereins Deutscher Ingenieure, 46(1524-1530):1572-1577, 1900.

[6] K H Roscoe, ANn Schofield, and CP Wroth. On the yielding of soils. Geotechnique, 8(1):22-53, 1958.

[7] Andrew Schofield and Peter Wroth. Critical state soil mechanics, volume 310. McGrawHill London, 1968.

[8] Ronald Midgley Nedderman. Statics and kinematics of granular materials. Cambridge University Press, 2005.

[9] Jun Ai. Particle scale and bulk scale investigation of granular piles and silos. The University of Edinburgh, 2010.

[10] James B Knight, Christopher G Fandrich, Chun Ning Lau, Heinrich M Jaeger, and Sidney R Nagel. Density relaxation in a vibrated granular material. Physical review E, 51(5):3957, 1995.

[11] Patrick Richard, Mario Nicodemi, Renaud Delannay, Philippe Ribiere, and Daniel Bideau. Slow relaxation and compaction of granular systems. Nature materials, 4(2):121, 2005 .

[12] Gianfranco D'Anna and Gerard Grémaud. The jamming route to the glass state in weakly perturbed granular media. Nature, 413(6854):407, 2001. 
[13] Farhang Radjai, Stéphane Roux, and Jean Jacques Moreau. Contact forces in a granular packing. Chaos: An Interdisciplinary Journal of Nonlinear Science, 9(3):544-550, 1999.

[14] Jaroslav Feda. Mechanics of particulate materials, volume 30. Elsevier, 2013.

[15] Dennis C Rapaport and Dennis C Rapaport Rapaport. The art of molecular dynamics simulation. Cambridge university press, 2004.

[16] A Drescher and G De Josselin De Jong. Photoelastic verification of a mechanical model for the flow of a granular material. Journal of the Mechanics and Physics of Solids, 20(5):337-340, 1972.

[17] Masanobu Oda and Junichi Konishi. Microscopic deformation mechanism of granular material in simple shear. Soils and foundations, 14(4):25-38, 1974.

[18] J Zhang, TS Majmudar, A Tordesillas, and RP Behringer. Statistical properties of a 2d granular material subjected to cyclic shear. Granular Matter, 12(2):159-172, 2010.

[19] ZX Yang, XS Li, and J Yang. Quantifying and modelling fabric anisotropy of granular soils. Géotechnique, 58(4):237-248, 2008.

[20] Sara Abedi, Amy L Rechenmacher, and Andrés D Orlando. Vortex formation and dissolution in sheared sands. Granular Matter, 14(6):695-705, 2012.

[21] Danuta Lesniewska and David Muir Wood. Observations of stresses and strains in a granular material. Journal of engineering mechanics, 135(9):1038-1054, 2009.

[22] A Rechenmacher, S Abedi, and O Chupin. Evolution of force chains in shear bands in sands. Geotechnique, 60(5):343, 2010.

[23] KH Roscoe. The determination of strains in soils by an x-ray method. Civ. Engng Publ. Wks Rev., 58:873-876, 1963.

[24] Joseph J Monaghan. An introduction to sph. Computer physics communications, 48(1):89-96, 1988.

[25] Joe J Monaghan. Smoothed particle hydrodynamics. Annual review of astronomy and astrophysics, 30(1):543-574, 1992.

[26] YT Feng, K Han, and DRJ Owen. Coupled lattice boltzmann method and discrete element modelling of particle transport in turbulent fluid flows: Computational issues. International Journal for Numerical Methods in Engineering, 72(9):1111-1134, 2007.

[27] Min Wang, YT Feng, and CY Wang. Coupled bonded particle and lattice boltzmann method for modelling fluid-solid interaction. International Journal for Numerical and Analytical Methods in Geomechanics, 40(10):1383-1401, 2016. 
[28] Min Wang, YT Feng, GN Pande, and TT Zhao. A coupled 3-dimensional bonded discrete element and lattice boltzmann method for fluid-solid coupling in cohesive geomaterials. International Journal for Numerical and Analytical Methods in Geomechanics, 42(12):1405-1424, 2018.

[29] Min Wang, YT Feng, DRJ Owen, and TM Qu. A novel algorithm of immersed moving boundary scheme for fluid-particle interactions in dem-lbm. Computer Methods in Applied Mechanics and Engineering, 346:109-125, 2019.

[30] BJ Alder and TEf Wainwright. Phase transition for a hard sphere system. The Journal of chemical physics, 27(5):1208-1209, 1957.

[31] Godehard Sutmann. Classical molecular dynamics and parallel computing. FZJ-ZAM, 2002.

[32] KA Alshibli and A Hasan. Spatial variation of void ratio and shear band thickness in sand using x-ray computed tomography. Géotechnique, 58(4):249-257, 2008.

[33] Ted Belytschko, Yury Krongauz, Daniel Organ, Mark Fleming, and Petr Krysl. Meshless methods: an overview and recent developments. Computer methods in applied mechanics and engineering, 139(1-4):3-47, 1996.

[34] Peter A Cundall and Otto DL Strack. A discrete numerical model for granular assemblies. geotechnique, 29(1):47-65, 1979.

[35] Lanru Jing and Ove Stephansson. Fundamentals of discrete element methods for rock engineering: theory and applications, volume 85. Elsevier, 2007.

[36] Otis R Walton and Robert L Braun. Viscosity, granular-temperature, and stress calculations for shearing assemblies of inelastic, frictional disks. Journal of rheology, 30(5):949-980, 1986.

[37] Xiaoshan Lin and T-T Ng. A three-dimensional discrete element model using arrays of ellipsoids. Geotechnique, 47(2):319-329, 1997.

[38] Yutaka Tsuji, Toshihiro Kawaguchi, and Toshitsugu Tanaka. Discrete particle simulation of two-dimensional fluidized bed. Powder technology, 77(1):79-87, 1993.

[39] David O Potyondy and PA Cundall. A bonded-particle model for rock. International journal of rock mechanics and mining sciences, 41(8):1329-1364, 2004.

[40] Jan Kozicki and Frederic V Donze. A new open-source software developed for numerical simulations using discrete modeling methods. Computer Methods in Applied Mechanics and Engineering, 197(49-50):4429-4443, 2008.

[41] Dion Weatherley. Esys-particle v2. 0 user's guide. 2009. 
[42] C Kloss and C Goniva. Liggghts: a new open source discrete element simulation software. In Proceedings of The Fifth International Conference on Discrete Element Methods, London, UK, pages 25-26, 2010.

[43] Engelbert Tijskens, Herman Ramon, and Josse De Baerdemaeker. Discrete element modelling for process simulation in agriculture. Journal of sound and vibration, 266(3):493$514,2003$.

[44] T Kawaguchi, T Tanaka, and Y Tsuji. Numerical simulation of two-dimensional fluidized beds using the discrete element method (comparison between the two-and three-dimensional models). Powder technology, 96(2):129-138, 1998.

[45] Wei Zhou, Jiaying Liu, Gang Ma, and Xiaolin Chang. Three-dimensional dem investigation of critical state and dilatancy behaviors of granular materials. Acta Geotechnica, $12(3): 527-540,2017$.

[46] Gang Ma, Richard A Regueiro, Wei Zhou, and Jiaying Liu. Spatiotemporal analysis of strain localization in dense granular materials. Acta Geotechnica, pages 1-18, 2018.

[47] Tongming Qu, Shuying Wang, Jinyang Fu, Qinxin Hu, and Xuemin Zhang. Numerical examination of epb shield tunneling-induced responses at various discharge ratios. Journal of Performance of Constructed Facilities, 33(3):04019035, 2019.

[48] Shuying Wang, Tongming Qu, Yong Fang, Jinyang Fu, and Junsheng Yang. Stress responses associated with earth pressure balance shield tunneling in dry granular ground using the discrete-element method. International Journal of Geomechanics, 19(7):04019060, 2019.

[49] Tongming Qu, Shuying Wang, Jinyang Fu, Qinxin Hu, and Junsheng Yang. Numerical investigation on muck pressures during epb shield tunneling with varying discharge ratio based on coupled pfc3d/flac3d method. In Civil Infrastructures Confronting Severe Weathers and Climate Changes Conference, pages 204-213. Springer, 2018.

[50] Tongming Qu, YT Feng, Yong Wang, and Min Wang. Discrete element modelling of flexible membrane boundaries for triaxial tests. Computers and Geotechnics, 115:103154, 2019 .

[51] Tongming Qu, Shuying Wang, and Qinxin Hu. Coupled discrete element-finite difference method for analysing effects of cohesionless soil conditioning on tunneling behaviour of epb shield. KSCE journal of Civil Engineering, In press.

[52] LUC Scholtès and Frédéric-Victor Donzé. Modelling progressive failure in fractured rock masses using a 3d discrete element method. International Journal of Rock Mechanics and Mining Sciences, 52:18-30, 2012. 
[53] William R Ketterhagen, Mary T am Ende, and Bruno C Hancock. Process modeling in the pharmaceutical industry using the discrete element method. Journal of pharmaceutical sciences, 98(2):442-470, 2009.

[54] Madhusudhan Kodam, Jennifer Curtis, Bruno Hancock, and Carl Wassgren. Discrete element method modeling of bi-convex pharmaceutical tablets: contact detection algorithms and validation. Chemical engineering science, 69(1):587-601, 2012.

[55] V. Šmilauer et al. Yade Documentation 2nd ed. The Yade Project, 2015. http://yadedem.org/doc/.

[56] D Weatherley, V Boros, and W Hancock. Esys-particle tutorial and user's guide version 2.1. Earth Systems Science Computational Centre, The University of Queensland, 2011.

[57] PFC3D Itasca. Particle flow code in 3 dimensions, user's guide, 2008.

[58] DEM Solutions. Edem 2.3 user guide. Edinburgh, Scotland, UK, 2010.

[59] https://www.rockfieldglobal.com.

[60] B Simpson and F Tatsuoka. Geotechnics: the next 60 years. Geotechnique, 58(5):357-368, 2008.

[61] AB Yu. Discrete element method: An effective way for particle scale research of particulate matter. Engineering Computations, 21(2/3/4):205-214, 2004.

[62] Rodrigo Gallego, Lluis Masanes, Gonzalo De La Torre, Chirag Dhara, Leandro Aolita, and Antonio Acín. Full randomness from arbitrarily deterministic events. Nature communications, 4:2654, 2013.

[63] Bruno Sudret and Armen Der Kiureghian. Stochastic finite element methods and reliability: a state-of-the-art report. Department of Civil and Environmental Engineering, University of California, 2000.

[64] JA Greenwood and JB Pl Williamson. Contact of nominally flat surfaces. Proc. R. Soc. Lond. A, 295(1442):300-319, 1966.

[65] Jacques Duran. Sands, powders, and grains: an introduction to the physics of granular materials. Springer Science \& Business Media, 2012.

[66] HP Zhu, ZY Zhou, RY Yang, and AB Yu. Discrete particle simulation of particulate systems: theoretical developments. Chemical Engineering Science, 62(13):3378-3396, 2007.

[67] Caroline Hogue. Shape representation and contact detection for discrete element simulations of arbitrary geometries. Engineering Computations, 15(3):374-390, 1998.

[68] GT Houlsby. Potential particles: a method for modelling non-circular particles in dem. Computers and Geotechnics, 36(6):953-959, 2009. 
[69] Paul W Cleary. Granular flows: fundamentals and applications. Granular and Complex Materials, pages 141-168, 2007.

[70] Leo Rothenburg and Richard J Bathurst. Numerical simulation of idealized granular assemblies with plane elliptical particles. Computers and geotechnics, 11(4):315-329, 1991.

[71] John M Ting. A robust algorithm for ellipse-based discrete element modelling of granular materials. Computers and Geotechnics, 13(3):175-186, 1992.

[72] Xiaoshan Lin and Tang-Tat Ng. Contact detection algorithms for three-dimensional ellipsoids in discrete element modelling. International Journal for Numerical and Analytical Methods in Geomechanics, 19(9):653-659, 1995.

[73] Tang-Tat Ng. Fabric evolution of ellipsoidal arrays with different particle shapes. Journal of engineering mechanics, 127(10):994-999, 2001.

[74] Peter A Cundall. Formulation of a three-dimensional distinct element model-part i. a scheme to detect and represent contacts in a system composed of many polyhedral blocks. International Journal of Rock Mechanics and Mining Sciences and Geomechanics Abstracts, 25(3):107-116, 1988.

[75] JF Favier, MH Abbaspour-Fard, M Kremmer, and AO Raji. Shape representation of axisymmetrical, non-spherical particles in discrete element simulation using multi-element model particles. Engineering computations, 16(4):467-480, 1999.

[76] Patricia A Thomas and Jonathan D Bray. Capturing nonspherical shape of granular media with disk clusters. Journal of Geotechnical and Geoenvironmental Engineering, 125(3):169-178, 1999.

[77] Nivedita Das, Patrick Giordano, Daniel Barrot, Shreekanth Mandayam, Beena Sukumaran, Alaa K Ashmawy, et al. Discrete element modeling and shape characterization of realistic granular shapes. In The Eighteenth International Offshore and Polar Engineering Conference. International Society of Offshore and Polar Engineers, 2008.

[78] X Garcia, J-P Latham, Jin-sheng XIANG, and JP Harrison. A clustered overlapping sphere algorithm to represent real particles in discrete element modelling. Geotechnique, 59(9):779-784, 2009.

[79] Y.T. Feng and D.R.J. Owen. Discrete element method - short course on particle based methods, barcelona, spain, 2008.

[80] Antonio A Munjiza. The combined finite-discrete element method. John Wiley \& Sons, 2004.

[81] YT Feng and DRJ Owen. An augmented spatial digital tree algorithm for contact detection in computational mechanics. International Journal for Numerical Methods in Engineering, 55(2):159-176, 2002. 
[82] K Han, YT Feng, and DRJ Owen. Performance comparisons of tree-based and cell-based contact detection algorithms. Engineering Computations, 24(2):165-181, 2007.

[83] Thorsten Pöschel and Thomas Schwager. Computational granular dynamics: models and algorithms. Springer Science \& Business Media, 2005.

[84] A Bobet, A Fakhimi, S Johnson, J Morris, F Tonon, and M Ronald Yeung. Numerical models in discontinuous media: review of advances for rock mechanics applications. Journal of Geotechnical and Geoenvironmental Engineering, 135(11):1547-1561, 2009.

[85] Paul W Cleary. Dem simulation of industrial particle flows: case studies of dragline excavators, mixing in tumblers and centrifugal mills. Powder Technology, 109(1-3):83104,2000 .

[86] Raymond D Mindlin. Elastic spheres in contact under varying oblique forces. J. Applied Mech., 20:327-344, 1953.

[87] Colin Thornton and KK Yin. Impact of elastic spheres with and without adhesion. Powder technology, 65(1-3):153-166, 1991.

[88] Loc Vu-Quoc, Xiang Zhang, and OR Walton. A 3-d discrete-element method for dry granular flows of ellipsoidal particles. Computer methods in applied mechanics and engineering, 187(3-4):483-528, 2000.

[89] Masanobu Oda. Fabric tensor for discontinuous geological materials. Soils and Foundations, 22(4):96-108, 1982.

[90] Kazuyoshi Iwashita and Masanobu Oda. Rolling resistance at contacts in simulation of shear band development by dem. Journal of engineering mechanics, 124(3):285-292, 1998.

[91] N Belheine, J-P Plassiard, F-V Donzé, F Darve, and A Seridi. Numerical simulation of drained triaxial test using 3d discrete element modeling. Computers and Geotechnics, 36(1-2):320-331, 2009.

[92] MJ Jiang, H-S Yu, and D Harris. A novel discrete model for granular material incorporating rolling resistance. Computers and Geotechnics, 32(5):340-357, 2005.

[93] Mingjing Jiang, Serge Leroueil, Hehua Zhu, Hai-Sui Yu, and Jean-Marie Konrad. Two-dimensional discrete element theory for rough particles. International Journal of Geomechanics, 9(1):20-33, 2009.

[94] BK Cook, MY Lee, AA DiGiovanni, DR Bronowski, ED Perkins, and JR Williams. Discrete element modeling applied to laboratory simulation of near-wellbore mechanics. International Journal of Geomechanics, 4(1):19-27, 2004.

[95] GR McDowell and O Harireche. Discrete element modelling of soil particle fracture. Géotechnique, 52(2):131-135, 2002. 
[96] PHSW Kulatilake, Bwalya Malama, and Jialai Wang. Physical and particle flow modeling of jointed rock block behavior under uniaxial loading. International Journal of Rock Mechanics and Mining Sciences, 38(5):641-657, 2001.

[97] Lok Yee Geraldine Cheung. Micromechanics of sand production in oil wells. PhD thesis, Imperial College London, 2010.

[98] EJ Abbott and FA Firestone. Specifying surface quality: a method based on accurate measurement and comparison. SPIE MILESTONE SERIES MS, 107:63-63, 1995.

[99] C Wang, DD Tannant, and PA Lilly. Numerical analysis of the stability of heavily jointed rock slopes using pfc2d. International Journal of Rock Mechanics and Mining Sciences, 40(3):415-424, 2003.

[100] A Fakhimi, JJ Riedel, and Joseph F Labuz. Shear banding in sandstone: Physical and numerical studies. International Journal of Geomechanics, 6(3):185-194, 2006.

[101] Olgierd Cecil Zienkiewicz, Robert Leroy Taylor, and Robert Leroy Taylor. The finite element method: solid mechanics, volume 2. Butterworth-heinemann, 2000.

[102] Catherine O'Sullivan. Particulate discrete element modelling: a geomechanics perspective. CRC Press, 2014.

[103] Kristin Lochmann, Luc Oger, and Dietrich Stoyan. Statistical analysis of random sphere packings with variable radius distribution. Solid State Sciences, 8(12):1397-1413, 2006.

[104] BA Klumov, SA Khrapak, and GE Morfill. Structural properties of dense hard sphere packings. Physical Review B, 83(18):184105, 2011.

[105] Xiang Song Li and Yannis F Dafalias. Dilatancy for cohesionless soils. Geotechnique, 50(4):449-460, 2000.

[106] Xin Huang, Kevin J Hanley, Catherine O'Sullivan, and Fiona CY Kwok. Effect of sample size on the response of dem samples with a realistic grading. Particuology, 15:107-115, 2014.

[107] Lia Papadopoulos, Mason A Porter, Karen E Daniels, and Danielle S Bassett. Network analysis of particles and grains. Journal of Complex Networks, 6(4):485-565, 2018.

[108] G Marketos and Malcolm D Bolton. Flat boundaries and their effect on sand testing. International Journal for numerical and analytical methods in geomechanics, 34(8):821$837,2010$.

[109] Chun-Yi Kuo and J David Frost. Uniformity evaluation of cohesionless specimens using digital image analysis. Journal of Geotechnical Engineering, 122(5):390-396, 1996.

[110] Matthew R Kuhn. Structured deformation in granular materials. Mechanics of materials, 31(6):407-429, 1999. 
[111] Colin Thornton. Numerical simulations of deviatoric shear deformation of granular media. Géotechnique, 50(1):43-53, 2000.

[112] Matthew R Kuhn. Heterogeneity and patterning in the quasi-static behavior of granular materials. Granular Matter, 4(4):155-166, 2003.

[113] M Oda, J Konishi, and S Nemat-Nasser. Some experimentally based fundamental results on the mechanical behaviour of granular materials. Geotechnique, 30(4):479-495, 1980.

[114] A Wouterse, Stefan Luding, and AP Philipse. On contact numbers in random rod packings. Granular Matter, 11(3):169-177, 2009.

[115] ME Barton. Cohesive sands: The natural transition from sands to sandstones. Geotechnical Engineering of Hard Soils and Soft Rocks, 1:367-374, 1993.

[116] J Fonseca, C O'Sullivan, and MR Coop. Quantitative description of grain contacts in a locked sand. 2010.

[117] L Rothenburg and Nicolaas P Kruyt. Critical state and evolution of coordination number in simulated granular materials. International Journal of Solids and Structures, 41(21):5763-5774, 2004.

[118] Farhang Radjai, Michel Jean, Jean-Jacques Moreau, and Stéphane Roux. Force distributions in dense two-dimensional granular systems. Physical review letters, 77(2):274, 1996.

[119] Colin Thornton. Force transmission in granular media. Kona Powder and Particle Journal, 15:81-90, 1997.

[120] Charles Voivret, Farhang Radjai, J-Y Delenne, and Moulay Saïd El Youssoufi. Multiscale force networks in highly polydisperse granular media. Physical review letters, 102(17):178001, 2009.

[121] Daniel Barreto Gonzalez. Numerical and experimental investigation into the behaviour of granular materials under generalised stress states. 2009 .

[122] Masanobu Oda. Initial fabrics and their relations to mechanical properties of granular material. Soils and foundations, 12(1):17-36, 1972.

[123] M Oda. Fabric tensor and its geometrical meaning. Introduction to mechanics of granular materials, 1999.

[124] Leo Rothenburg and RJ Bathurst. Analytical study of induced anisotropy in idealized granular materials. Geotechnique, 39(4):601-614, 1989.

[125] M Satake. Constitution of mechanics of granular materials through graph representation. Theoretical and applied mechanics, 26:257-266, 1978. 
[126] M Satake. Fabric tensor in granular materials. In IUTAM Conference on Deformation and Flow of Granular Materials, 1982, pages 63-68. AA Balkema, 1982.

[127] G Thomas Mase, Ronald E Smelser, and George E Mase. Continuum mechanics for engineers. CRC press, 2009.

[128] JP Bardet. Observations on the effects of particle rotations on the failure of idealized granular materials. Mechanics of materials, 18(2):159-182, 1994.

[129] D Barreto, C O'Sullivan, and L Zdravkovic. Quantifying the evolution of soil fabric under different stress paths. In AIP Conference Proceedings, volume 1145, pages 181-184. AIP, 2009.

[130] NH Woodcock. Specification of fabric shapes using an eigenvalue method. Geological Society of America Bulletin, 88(9):1231-1236, 1977.

[131] Masanobu Oda, Siavouche Nemat-Nasser, and Junichi Konishi. Stress-induced anisotropy in granular masses. Soils and foundations, 25(3):85-97, 1985.

[132] Tang-Tat Ng. Discrete element method simulations of the critical state of a granular material. International Journal of Geomechanics, 9(5):209-216, 2009.

[133] Krste Asanovic, Ras Bodik, Bryan Christopher Catanzaro, Joseph James Gebis, Parry Husbands, Kurt Keutzer, David A Patterson, William Lester Plishker, John Shalf, Samuel Webb Williams, et al. The landscape of parallel computing research: A view from berkeley. Technical report, Technical Report UCB/EECS-2006-183, EECS Department, University of California, Berkeley, 2006.

[134] Keren Bergman, Shekhar Borkar, Dan Campbell, William Carlson, William Dally, Monty Denneau, Paul Franzon, William Harrod, Kerry Hill, Jon Hiller, et al. Exascale computing study: Technology challenges in achieving exascale systems. Defense Advanced Research Projects Agency Information Processing Techniques Office (DARPA IPTO), Tech. Rep, 15, 2008.

[135] Ghassan Shahin, Jacques Desrues, Stefano Dal Pont, Gaël Combe, and Albert Argilaga. A study of the influence of rev variability in double-scale fem $\times$ dem analysis. International Journal for Numerical Methods in Engineering, 107(10):882-900, 2016.

[136] M Kazari. A study on conditions for similarity of particle motion in numerical simulation of dense gas-solid two phase flow. In Proceedings of the 2nd International Conference on Multiphase Flow'95, Kyoto, 1995.

[137] Kimiaki Washino, Chih-Hung Hsu, Toshihiro Kawaguchi, and Yutaka Tsuji. Similarity model for dem simulation of fluidized bed. Journal of the Society of Powder Technology, Japan, 44(3):198-205, 2007.

[138] M Sakano. Numerical simulation of two-dimensional fluidized bed using discrete element method with imaginary sphere model. Japanese J. Multiphase Flow, 14:66-73, 2000. 
[139] K Kuwagi. The similar particle assembly (spa) model, an approach for large-scale discrete element (dem) simulation. Fluidization, pages 243-250, 2004.

[140] Mikio Sakai and Seiichi Koshizuka. Large-scale discrete element modeling in pneumatic conveying. Chemical Engineering Science, 64(3):533-539, 2009.

[141] MA Mokhtar, K Kuwagi, T Takami, H Hirano, and M Horio. Validation of the similar particle assembly (spa) model for the fluidization of geldart's group a and d particles. AIChE Journal, 58(1):87-98, 2012.

[142] Mikio Sakai, Yoshinori Yamada, Yusuke Shigeto, Kazuya Shibata, Vanessa M Kawasaki, and Seiichi Koshizuka. Large-scale discrete element modeling in a fluidized bed. International Journal for Numerical Methods in Fluids, 64(10-12):1319-1335, 2010.

[143] M Ricklick and O Baran. Analytical scaling of dem particles for efficient packedbed simulations. International Conference on Heat Transfer, Fluid Mechanics and Thermodynamics, 2014.

[144] James E Hilton and Paul W Cleary. Comparison of non-cohesive resolved and coarse grain dem models for gas flow through particle beds. Applied Mathematical Modelling, 38(17-18):4197-4214, 2014.

[145] Liqiang Lu, Kisoo Yoo, and Sofiane Benyahia. Coarse-grained-particle method for simulation of liquid-solids reacting flows. Industrial $\mathcal{E}$ Engineering Chemistry Research, 55(39):10477-10491, 2016.

[146] Kaiwei Chu, Jiang Chen, and Aibing Yu. Applicability of a coarse-grained cfd-dem model on dense medium cyclone. Minerals Engineering, 90:43-54, 2016.

[147] Husam A Elghannay and Danesh K Tafti. Evaluation of coarse graining dem using representative particle model. In International Conference on Discrete Element Methods, pages 57-65. Springer, 2016.

[148] K Kaneko, Kenjiro Terada, Takashi Kyoya, and Y Kishino. Global-local analysis of granular media in quasi-static equilibrium. International Journal of Solids and Structures, 40(15):4043-4069, 2003.

[149] C Miehe, J Dettmar, and D Zäh. Homogenization and two-scale simulations of granular materials for different microstructural constraints. International Journal for Numerical Methods in Engineering, 83(8-9):1206-1236, 2010.

[150] Jose E Andrade, CF Avila, SA Hall, Nicolas Lenoir, and Gioacchino Viggiani. Multiscale modeling and characterization of granular matter: from grain kinematics to continuum mechanics. Journal of the Mechanics and Physics of Solids, 59(2):237-250, 2011.

[151] Carlos Avila and José Andrade. Advances in multiscale modeling and characterization of granular matter. Procedia IUTAM, 3:157-171, 2012. 
[152] Michał Nitka, Bilbie Bilbie, Gaël Combe, Cristian Dascalu, and Jacques Desrues. A micro-macro (dem-fem) model of the behavior of granular solids. In 1st International Symposium on Computational Geomechanics (ComGeo I), Juan-les-Pins, France, pages 38-48, 2009.

[153] TK Nguyen, G Combe, D Caillerie, and J Desrues. Modeling of a cohesive granular materials by a multi-scale approach. In AIP Conference Proceedings, volume 1542, pages 1194-1197. AIP, 2013.

[154] Ning Guo and Jidong Zhao. A coupled fem/dem approach for hierarchical multiscale modelling of granular media. International Journal for Numerical Methods in Engineering, 99(11):789-818, 2014.

[155] J Desrues, TK Nguyen, G Combe, and D Caillerie. Fem $\times$ dem multi-scale analysis of boundary value problems involving strain localization. In International Workshop on Bifurcation and Degradation in Geomaterials, pages 259-265. Springer, 2014.

[156] Siavouche Nemat-Nasser and Muneo Hori. Micromechanics: overall properties of heterogeneous materials, volume 37. Elsevier, 2013.

[157] S Luding, M Lätzel, W Volk, S Diebels, and HJ Herrmann. From discrete element simulations to a continuum model. Computer methods in applied mechanics and engineering, 191(1-2):21-28, 2001.

[158] Wei Zhou, Gang Ma, Xiao-Lin Chang, and Yin Duan. Discrete modeling of rockfill materials considering the irregular shaped particles and their crushability. Engineering Computations, 32(4):1104-1120, 2015.

[159] Richard P Jensen, Peter J Bosscher, Michael E Plesha, and Tuncer B Edil. Dem simulation of granular media - structure interface: effects of surface roughness and particle shape. International Journal for Numerical and Analytical Methods in Geomechanics, 23(6):531-547, 1999 .

[160] Linbing Wang, Jin-Young Park, and Yanrong Fu. Representation of real particles for dem simulation using x-ray tomography. Construction and Building Materials, 21(2):338-346, 2007.

[161] MMGR Lu and GR McDowell. The importance of modelling ballast particle shape in the discrete element method. Granular matter, 9(1-2):69, 2007.

[162] J-F Ferellec and GR McDowell. A simple method to create complex particle shapes for dem. Geomechanics and Geoengineering: An International Journal, 3(3):211-216, 2008.

[163] MM Mollanouri Shamsi and AA Mirghasemi. Numerical simulation of 3d semi-realshaped granular particle assembly. Powder technology, 221:431-446, 2012.

[164] JR Barber and M Ciavarella. Contact mechanics. International Journal of solids and structures, 37(1-2):29-43, 2000. 
[165] Jim A Greenwood and J Hl Tripp. The elastic contact of rough spheres. Journal of Applied Mechanics, 34(1):153-159, 1967.

[166] JF Archard. Elastic deformation and the laws of friction. Proc. R. Soc. Lond. A, 243(1233):190-205, 1957.

[167] Arunava Majumdar and Bharat Bhushan. Role of fractal geometry in roughness characterization and contact mechanics of surfaces. Journal of tribology, 112(2):205-216, 1990.

[168] HM Stanley and T Kato. An fft-based method for rough surface contact. Journal of tribology, 119(3):481-485, 1997.

[169] Kenneth Langstreth Johnson. Contact mechanics. Cambridge university press, 1987.

[170] WR Chang, I Etsion, and D BASME Bogy. An elastic-plastic model for the contact of rough surfaces. Journal of tribology, 109(2):257-263, 1987.

[171] Robert L Jackson and Lior Kogut. A comparison of flattening and indentation approaches for contact mechanics modeling of single asperity contacts. Journal of Tribology, 128(1):209-212, 2006.

[172] Milton Abramowitz and Irene A Stegun. Handbook of mathematical functions: with formulas, graphs, and mathematical tables, volume 55. Courier Corporation, 1965.

[173] Ali Beheshti and MM Khonsari. Asperity micro-contact models as applied to the deformation of rough line contact. Tribology International, 52:61-74, 2012.

[174] Ali Beheshti and MM Khonsari. On the contact of curved rough surfaces: contact behavior and predictive formulas. Journal of Applied Mechanics, 81(11):111004, 2014.

[175] Yongwu Zhao, David M Maietta, and L Chang. An asperity microcontact model incorporating the transition from elastic deformation to fully plastic flow. Journal of Tribology, 122(1):86-93, 2000.

[176] Z Ning and C Thornton. Elastic-plastic impact of fine particles with a surface. Powders and grains, 93:33-38, 1993.

[177] C Thornton. Coefficient of restitution for collinear collisions of elastic-perfectly plastic spheres. Journal of Applied Mechanics, 64(2):383-386, 1997.

[178] Lior Kogut and Izhak Etsion. Elastic-plastic contact analysis of a sphere and a rigid flat. Journal of applied Mechanics, 69(5):657-662, 2002.

[179] Lior Kogut and Izhak Etsion. A finite element based elastic-plastic model for the contact of rough surfaces. Tribology transactions, 46(3):383-390, 2003.

[180] Robert L Jackson and Itzhak Green. A finite element study of elasto-plastic hemispherical contact against a rigid flat. Journal of tribology, 127(2):343-354, 2005. 
[181] Daolin Ma and Caishan Liu. Contact law and coefficient of restitution in elastoplastic spheres. Journal of Applied Mechanics, 82(12):121006, 2015.

[182] Hui Wang, Xiaochun Yin, Xiaoli Qi, Qingming Deng, Bo Yu, and Qiming Hao. Experimental and theoretical analysis of the elastic-plastic normal repeated impacts of a sphere on a beam. International Journal of Solids and Structures, 109:131-142, 2017.

[183] Loc Vu-Quoc and Xiang Zhang. An elastoplastic contact force-displacement model in the normal direction: displacement-driven version. In Proceedings of the Royal Society of London A: Mathematical, Physical and Engineering Sciences, volume 455, pages 4013-4044. The Royal Society, 1999.

[184] Hamid Ghaednia, Sara A Pope, Robert L Jackson, and Dan B Marghitu. A comprehensive study of the elasto-plastic contact of a sphere and a flat. Tribology International, 93:78-90, 2016.

[185] HA Francis. Phenomenological analysis of plastic spherical indentation. Journal of Engineering Materials and technology, 98(3):272-281, 1976.

[186] G Amontons. De la resistance causee dansles machines. Mem. de l'Academie Royal A, pages $275-282$.

[187] C Coulomb. Theorie des machines simples, moeoires de mathematique et de physique. Academie des Sciences, 10:161-331, 1785.

[188] Leonhard Euler. Sur le frottement des corps solides. Mémoires de l'Académie des Sciences de Berlin, pages 122-132, 1750.

[189] Ian Hutchings and Philip Shipway. Tribology: friction and wear of engineering materials. Butterworth-Heinemann, 2017.

[190] David Tabor. Friction - the present state of our understanding. Journal of lubrication technology, 103(2):169-179, 1981.

[191] Wen-Ruey Chang, I Etsion, and DB Bogy. Static friction coefficient model for metallic rough surfaces. Journal of tribology, 110(1):57-63, 1988.

[192] G_M_Hamilton. Explicit equations for the stresses beneath a sliding spherical contact. Proceedings of the Institution of Mechanical Engineers, Part C: Journal of Mechanical Engineering Science, 197(1):53-59, 1983.

[193] SK Roy Chowdhury and P Ghosh. Adhesion and adhesional friction at the contact between solids. Wear, 174(1-2):9-19, 1994.

[194] AR Savkoor and GAD Briggs. The effect of tangential force on the contact of elastic solids in adhesion. Proceedings of the Royal Society of London. A. Mathematical and Physical Sciences, 356(1684):103-114, 1977. 
[195] Ajay K Waghmare and Prasanta Sahoo. Friction analysis at elastic-plastic contact of rough surfaces using n-point asperity model. Proceedings of the Institution of Mechanical Engineers, Part J: Journal of Engineering Tribology, 230(10):1258-1272, 2016.

[196] Prasanta Sahoo, Anirban Mitra, and Kashinath Saha. Elastic-plastic adhesive contact of rough surfaces using n-point asperity model. Journal of Physics D: Applied Physics, 42(6):065302, 2009.

[197] Kenneth Langstreth Johnson, Kevin Kendall, and AD Roberts. Surface energy and the contact of elastic solids. Proceedings of the royal society of London. A. mathematical and physical sciences, 324(1558):301-313, 1971.

[198] Majid Bahrami, M Michael Yovanovich, and J Richard Culham. Thermal joint resistances of nonconforming rough surfaces with gas-filled gaps. Journal of Thermophysics and heat transfer, 18(3):326-332, 2004.

[199] MG Cooper, BB Mikic, and MM Yovanovich. Thermal contact conductance. International Journal of heat and mass transfer, 12(3):279-300, 1969.

[200] Adrian Bejan and Allan D Kraus. Heat transfer handbook, volume 1. John Wiley \& Sons, 2003.

[201] YZ Li, CV Madhusudana, and E Leonardi. Experimental investigation of thermal contact conductance: Variations of surface microhardness and roughness. International journal of thermophysics, 19(6):1691-1704, 1998.

[202] Borivoje Mikić. Thermal constriction resistance due to non-uniform surface conditions; contact resistance at non-uniform interface pressure. International Journal of Heat and Mass Transfer, 13(9):1497-1500, 1970.

[203] MA Lambert and LS Fletcher. Thermal contact conductance of spherical rough metals. Journal of heat transfer, 119(4):684-690, 1997.

[204] YT Feng, K Han, and DRJ Owen. Filling domains with disks: an advancing front approach. International journal for numerical methods in engineering, 56(5):699-713, 2003.

[205] R. Löhner and E Oñate. A general advancing front technique for filling space with arbitrary objects. International journal for numerical methods in engineering, 61(12):19771991, 2004.

[206] K Han, YT Feng, and DRJ Owen. Sphere packing with a geometric based compression algorithm. Powder Technology, 155(1):33-41, 2005.

[207] John-Paul Latham, Ante Munjiza, and Yang Lu. On the prediction of void porosity and packing of rock particulates. Powder Technology, 125(1):10-27, 2002. 
[208] Y Sheng, CJ Lawrence, BJ Briscoe, and C Thornton. Numerical studies of uniaxial powder compaction process by 3d dem. Engineering Computations, 21(2/3/4):304-317, 2004 .

[209] Romain Guises, Jiansheng Xiang, John-Paul Latham, and Antonio Munjiza. Granular packing: numerical simulation and the characterisation of the effect of particle shape. Granular Matter, 11(5):281-292, 2009.

[210] Jean-François Jerier, Didier Imbault, Frederic-Victor Donze, and Pierre Doremus. A geometric algorithm based on tetrahedral meshes to generate a dense polydisperse sphere packing. Granular Matter, 11(1):43-52, 2009.

[211] XM Sun, YJ Dong, PF Hao, L Shi, F Li, and YT Feng. Three-dimensional numerical simulation of quasi-static pebble flow. Advanced Powder Technology, 28(2):499-505, 2017.

[212] B Cambou, Ph Dubujet, and C Nouguier-Lehon. Anisotropy in granular materials at different scales. Mechanics of materials, 36(12):1185-1194, 2004.

[213] Harrison H Barrett and Kyle J Myers. Foundations of image science. John Wiley \& Sons, 2013.

[214] Rafael C Gonzales and Richard E Woods. Digital image processing. Prentice Hall, second edition. edition, 2001.

[215] Simon Haykin and Richard Lippmann. Neural networks, a comprehensive foundation. International journal of neural systems, 5(4):363-364, 1994.

[216] Maria Petrou and Costas Petrou. Image Processing: The Fundamentals. John Wiley and Sons, Ltd, Chichester, UK, 2011.

[217] I. T Jolliffe. Principal Component Analysis. Springer New York, New York, NY, second edition. edition, 2002.

[218] Hervé Abdi and Lynne J Williams. Principal component analysis. Wiley interdisciplinary reviews: computational statistics, 2(4):433-459, 2010.

[219] Gene H Golub et al. Cf van loan, matrix computations. The Johns Hopkins, 1996.

[220] YT Feng and DRJ Owen. Discrete element modelling of large scale particle systems - i: exact scaling laws. Computational Particle Mechanics, 1(2):159-168, 2014.

[221] YT Feng, Kuanjin Han, DRJ Owen, and J Loughran. On upscaling of discrete element models: similarity principles. Engineering Computations, 26(6):599-609, 2009.

[222] Nicolaas P Kruyt. Statics and kinematics of discrete cosserat-type granular materials. International journal of solids and structures, 40(3):511-534, 2003.

[223] David Muir Wood. Geotechnical modelling. CRC press, 2014. 
[224] Thorsten Pöschel, Clara Saluena, and Thomas Schwager. Scaling properties of granular materials. pages 173-184, 2001.

[225] Mikio Sakai, Hiroyuki Takahashi, Christopher C Pain, John-Paul Latham, and Jiansheng Xiang. Study on a large-scale discrete element model for fine particles in a fluidized bed. Advanced Powder Technology, 23(5):673-681, 2012.

[226] C Bierwisch, T Kraft, H Riedel, and M Moseler. Three-dimensional discrete element models for the granular statics and dynamics of powders in cavity filling. Journal of the Mechanics and Physics of Solids, 57(1):10-31, 2009.

[227] Stefan Radl, Charles Radeke, Johannes G Khinast, and Sankaran Sundaresan. Parcelbased approach for the simulation of gas-particle flows. In 8th International Conference on CFD in Oil \& Gas, Metallurgical and Process Industries, Trondheim, volume 2011, 2011.

[228] Daniel Schiochet Nasato, Christoph Goniva, Stefan Pirker, and Christoph Kloss. Coarse graining for large-scale dem simulations of particle flow-an investigation on contact and cohesion models. Procedia engineering, 102:1484-1490, 2015.

[229] Subhash C Thakur, Jin Y Ooi, and Hossein Ahmadian. Scaling of discrete element model parameters for cohesionless and cohesive solid. Powder technology, 293:130-137, 2016.

[230] Taeyoung Yun and Heemun Park. Applicability evaluation of similarity principle in discrete element analysis. In International Conference on Discrete Element Methods, pages 117-123. Springer, 2016.

[231] María Asunción Izquierdo Barrientos. Heat transfer and thermal storage in fixed and fluidized beds of phase change materials. 2014.

[232] T Saitoh and K Hirose. High-performance phase-change thermal energy storage using spherical capsules. Chemical Engineering Communications, 41(1-6):39-58, 1986.

[233] Frédéric Kuznik, Damien David, Kevyn Johannes, and Jean-Jacques Roux. A review on phase change materials integrated in building walls. Renewable and Sustainable Energy Reviews, 15(1):379-391, 2011.

[234] MK Anuar Sharif, AA Al-Abidi, Sohif Mat, Kamaruzzaman Sopian, Mohd Hafidz Ruslan, MY Sulaiman, and MAM Rosli. Review of the application of phase change material for heating and domestic hot water systems. Renewable and Sustainable Energy Reviews, 42:557-568, 2015.

[235] Xiwen Cheng, Xiaoqiang Zhai, and Ruzhu Wang. Thermal performance analysis of a packed bed cold storage unit using composite pcm capsules for high temperature solar cooling application. Applied thermal engineering, 100:247-255, 2016. 
[236] Hao Peng, Rui Li, Xiang Ling, and Huihua Dong. Modeling on heat storage performance of compressed air in a packed bed system. Applied energy, 160:1-9, 2015.

[237] Keumnam Cho and SH Choi. Thermal characteristics of paraffin in a spherical capsule during freezing and melting processes. International journal of heat and mass transfer, 43(17):3183-3196, 2000.

[238] Eduard Oró, Albert Castell, Justin Chiu, Viktoria Martin, and Luisa F Cabeza. Stratification analysis in packed bed thermal energy storage systems. Applied energy, 109:476-487, 2013.

[239] D Vortmeyer and RJ Schaefer. Equivalence of one-and two-phase models for heat transfer processes in packed beds: one dimensional theory. Chemical Engineering Science, 29(2):485-491, 1974.

[240] To EW Schumann. Heat transfer: a liquid flowing through a porous prism. Journal of the Franklin Institute, 208(3):405-416, 1929.

[241] Alvaro de Gracia and Luisa F Cabeza. Numerical simulation of a pcm packed bed system: a review. Renewable and Sustainable Energy Reviews, 69:1055-1063, 2017.

[242] Ciril Arkar and Sašo Medved. Influence of accuracy of thermal property data of a phase change material on the result of a numerical model of a packed bed latent heat storage with spheres. Thermochimica Acta, 438(1-2):192-201, 2005.

[243] KAR Ismail and JR Henrıquez. Numerical and experimental study of spherical capsules packed bed latent heat storage system. Applied Thermal Engineering, 22(15):1705-1716, 2002.

[244] Yvan Dutil, Daniel R Rousse, Nizar Ben Salah, Stéphane Lassue, and Laurent Zalewski. A review on phase-change materials: mathematical modeling and simulations. Renewable and sustainable Energy reviews, 15(1):112-130, 2011.

[245] ML Hunt. Discrete element simulations for granular material flows: effective thermal conductivity and self-diffusivity. International journal of heat and mass transfer, 40(13):3059-3068, 1997.

[246] Watson L Vargas and JJ McCarthy. Heat conduction in granular materials. AIChE Journal, 47(5):1052-1059, 2001.

[247] Watson L Vargas and JJ McCarthy. Stress effects on the conductivity of particulate beds. Chemical Engineering Science, 57(15):3119-3131, 2002.

[248] Bodhisattwa Chaudhuri, Fernando J Muzzio, and M Silvina Tomassone. Modeling of heat transfer in granular flow in rotating vessels. Chemical Engineering Science, 61(19):6348-6360, 2006. 
[249] YT Feng, K Han, CF Li, and DRJ Owen. Discrete thermal element modelling of heat conduction in particle systems: Basic formulations. Journal of Computational Physics, 227(10):5072-5089, 2008.

[250] YT Feng, K Han, and DRJ Owen. Discrete thermal element modelling of heat conduction in particle systems: pipe-network model and transient analysis. Powder Technology, 193(3):248-256, 2009.

[251] John C Steuben, Athanasios P Iliopoulos, and John G Michopoulos. Discrete element modeling of particle-based additive manufacturing processes. Computer Methods in Applied Mechanics and Engineering, 305:537-561, 2016.

[252] HW Zhang, Q Zhou, HL Xing, and H Muhlhaus. A dem study on the effective thermal conductivity of granular assemblies. Powder technology, 205(1-3):172-183, 2011.

[253] Yuanbo Liang, Haiying Niu, and Yafei Lou. Expression for etc of the solid phase of randomly packed granular materials. Applied Thermal Engineering, 109:44-52, 2016.

[254] Akhil Reddy Peeketi, Marigrazia Moscardini, Akhil Vijayan, Yixiang Gan, Marc Kamlah, and Ratna Kumar Annabattula. Effective thermal conductivity of a compacted pebble bed in a stagnant gaseous environment: An analytical approach together with dem. Fusion Engineering and Design, 130:80-88, 2018.

[255] Yanio E Milian, Andrea Gutierrez, Mario Grageda, and Svetlana Ushak. A review on encapsulation techniques for inorganic phase change materials and the influence on their thermophysical properties. Renewable and Sustainable Energy Reviews, 73:983-999, 2017.

[256] A Jamekhorshid, SM Sadrameli, and Mohammed Farid. A review of microencapsulation methods of phase change materials (pcms) as a thermal energy storage (tes) medium. Renewable and Sustainable Energy Reviews, 31:531-542, 2014.

[257] Mohamed Rady. Granular phase change materials for thermal energy storage: experiments and numerical simulations. Applied Thermal Engineering, 29(14-15):3149-3159, 2009 .

[258] L Xia, P Zhang, and RZ Wang. Numerical heat transfer analysis of the packed bed latent heat storage system based on an effective packed bed model. Energy, 35(5):2022-2032, 2010.

[259] KAR Ismail and R Stuginsky Jr. A parametric study on possible fixed bed models for pcm and sensible heat storage. Applied Thermal Engineering, 19(7):757-788, 1999.

[260] Selvan Bellan, Tanvir E Alam, José González-Aguilar, Manuel Romero, Muhammad M Rahman, D Yogi Goswami, and Elias K Stefanakos. Numerical and experimental studies on heat transfer characteristics of thermal energy storage system packed with molten salt pcm capsules. Applied thermal engineering, 90:970-979, 2015. 
[261] JGH Borkink and KR Westerterp. Influence of tube and particle diameter on heat transport in packed beds. AIChE journal, 38(5):703-715, 1992.

[262] George Keith Batchelor and RW O'brien. Thermal or electrical conduction through a granular material. Proc. R. Soc. Lond. A, 355(1682):313-333, 1977.

[263] Watson L Vargas and Joseph J McCarthy. Thermal expansion effects and heat conduction in granular materials. Physical Review E, 76(4):041301, 2007.

[264] Xiao-Liang Wang and Dong-Yun Bai. Thermal expansion and thermal fluctuation effects in a binary granular mixture. International Journal of Heat and Mass Transfer, 116:84-92, 2018.

[265] Xiao-Liang Wang and Dong-Yun Bai. Thermal cycling leads grains to more homogeneous force networks and energy repartition. Powder technology, 339:111-118, 2018.

[266] M Michael Yovanovich. Four decades of research on thermal contact, gap, and joint resistance in microelectronics. IEEE transactions on components and packaging technologies, 28(2):182-206, 2005.

[267] BB Mikić. Thermal contact conductance; theoretical considerations. International Journal of Heat and Mass Transfer, 17(2):205-214, 1974.

[268] Yuanbo Liang and Xikui Li. A new model for heat transfer through the contact network of randomly packed granular material. Applied Thermal Engineering, 73(1):984-992, 2014.

[269] Shazim Ali Memon. Phase change materials integrated in building walls: A state of the art review. Renewable and sustainable energy reviews, 31:870-906, 2014.

[270] S Tavman and IH Tavman. Measurement of effective thermal conductivity of wheat as a function of moisture content. International communications in heat and mass transfer, 25(5):733-741, 1998.

[271] Zhou Zhao, KM Feng, and YJ Feng. Theoretical calculation and analysis modeling for the effective thermal conductivity of li4sio4 pebble bed. Fusion Engineering and Design, 85(10-12):1975-1980, 2010.

[272] Katalin Bagi. Stress and strain in granular assemblies. Mechanics of materials, 22(3):165$177,1996$.

[273] W Ehlers, E Ramm, S Diebels, and GA d'Addetta. From particle ensembles to cosserat continua: homogenization of contact forces towards stresses and couple stresses. International Journal of Solids and Structures, 40(24):6681-6702, 2003.

[274] Ching S Chang and Matthew R Kuhn. On virtual work and stress in granular media. International Journal of Solids and Structures, 42(13):3773-3793, 2005. 
[275] XH Wen, LJ Durlofsky, and MG Edwards. Use of border regions for improved permeability upscaling. Mathematical Geology, 35(5):521-547, 2003.

[276] J Stefan. Uber einige probleme der theorie der warmeletung. Sitzer. Wien. Akad. Math. Naturw., 98:473-484, 1889.

[277] Marek Błasik. Numerical scheme for the one-phase 1d stefan problem using curvilinear coordinates. Scientific Research of the Institute of Mathematics and Computer Science, 11(3):9-14, 2012.

[278] HS Carslaw and JC Jaeger. Conduction of heat in solids: Oxford Science Publications. Oxford, England, 1959.

[279] A Felix Regin, SC Solanki, and JS Saini. An analysis of a packed bed latent heat thermal energy storage system using pcm capsules: Numerical investigation. Renewable energy, 34(7):1765-1773, 2009.

[280] RG Craig, JD Eick, and FA Peyton. Properties of natural waxes used in dentistry. Journal of Dental Research, 44(6):1308-1316, 1965.

[281] J Lanier and M Jean. Experiments and numerical simulations with 2d disks assembly. Powder Technology, 109(1-3):206-221, 2000.

[282] Ryosuke Kitamura. Analysis of deformation mechanism of particulate material at particle scale. Soils and Foundations, 21(2):85-98, 1981.

[283] Catherine O'Sullivan and Jonathan D Bray. Selecting a suitable time step for discrete element simulations that use the central difference time integration scheme. Engineering Computations, 21(2/3/4):278-303, 2004.

[284] Ted Belytschko, Wing Kam Liu, Brian Moran, and Khalil Elkhodary. Nonlinear finite elements for continua and structures. John wiley \& sons, 2013.

[285] Tang-Tat Ng. Particle shape effect on macro-and micro-behaviors of monodisperse ellipsoids. International journal for numerical and analytical methods in geomechanics, $33(4): 511-527,2009$.

[286] T Matsushima and K Konagai. Grain-shape effect on peak strength of granular materials. Proc. 10 IACMAG: Computer Methods and Advances in Geomechanics, pages 361-366, 2001.

[287] Erfan G Nezami, Youssef MA Hashash, Dawei Zhao, and Jamshid Ghaboussi. Simulation of front end loader bucket-soil interaction using discrete element method. International journal for numerical and analytical methods in geomechanics, 31(9):1147-1162, 2007.

[288] Mingfei Lu and Glenn R McDowell. Discrete element modelling of railway ballast under triaxial conditions. Geomechanics and Geoengineering: An International Journal, $3(4): 257-270,2008$. 
[289] KD Kafui and C Thornton. Numerical simulations of impact breakage of a spherical crystalline agglomerate. Powder Technology, 109(1-3):113-132, 2000.

[290] Colin Thornton and Lianfeng Liu. How do agglomerates break? Powder Technology, 143:110-116, 2004.

[291] YP Cheng, Y Nakata, and MD Bolton. Discrete element simulation of crushable soil. Geotechnique, 53(7):633-641, 2003.

[292] S Utili and R Nova. Dem analysis of bonded granular geomaterials. International Journal for Numerical and Analytical Methods in Geomechanics, 32(17):1997-2031, 2008 .

[293] Sébastien Hentz, Laurent Daudeville, and Frédéric V Donzé. Identification and validation of a discrete element model for concrete. Journal of engineering mechanics, 130(6):709$719,2004$.

[294] Nicolaas P Kruyt and L Rothenburg. Plasticity of granular materials: a structuralmechanics view. In AIP conference proceedings, volume 1145, pages 1073-1076. AIP, 2009 .

[295] S Haeri, Y Wang, O Ghita, and J Sun. Discrete element simulation and experimental study of powder spreading process in additive manufacturing. Powder Technology, 306:45-54, 2017.

[296] Mohamad Muhieddine, Edouard Canot, and Ramiro March. Various approaches for solving problems in heat conduction with phase change. International Journal on Finite Volumes, page 19, 2009.

[297] Nacer Sadoun, EK Si-Ahmed, and Jack Legrand. On heat conduction with phase change: Accurate explicit numerical method. Journal of Applied Fluid Mechanics, 5(1), 2012.

[298] William D Murray. Numerical and machine solutions of transient heat conduction problems involving phase change. Journal of Heat Transfer, 81:106-112, 1959.

[299] S Kutluay, AR Bahadir, and A Özdeş. The numerical solution of one-phase classical stefan problem. Journal of computational and applied mathematics, 81(1):135-144, 1997.

[300] NS Asaithambi. A variable time step galerkin method for a one-dimensional stefan problem. Applied Mathematics and Computation, 81(2-3):189-200, 1997.

[301] Theodore R Goodman. The heat balance integral and its application to problems involving change of phase. Trans. ASME, J. Heat Transf., 80:335, 1958.

[302] H Go Landau. Heat conduction in a melting solid. Quarterly of Applied Mathematics, 8(1):81-94, 1950. 
[303] S Kutluay and A Esen. An isotherm migration formulation for one-phase stefan problem with a time dependent neumann condition. Applied Mathematics and computation, 150(1):59-67, 2004.

[304] Alaattin Esen and Selçuk Kutluay. A numerical solution of the stefan problem with a neumann-type boundary condition by enthalpy method. Applied Mathematics and Computation, 148(2):321-329, 2004.

[305] Comini Bonacina, G Comini, A Fasano, and M Primicerio. Numerical solution of phasechange problems. International Journal of Heat and Mass Transfer, 16(10):1825-1832, 1973 . 